\title{
Vocabulary Growth in Armenian-English Bilingual Kindergarteners
}

\author{
by
}

Alice Hovsepian

A thesis submitted in conformity with the requirements

for the degree of Doctor of Philosophy

Department of Speech-Language Pathology

University of Toronto

(C) Copyright by Alice Hovsepian « 2012 » 


\title{
Vocabulary Growth in Armenian-English Bilingual Kindergarteners
}

\author{
Alice Hovsepian \\ Doctor of Philosophy \\ Department of Speech-Language Pathology \\ University of Toronto \\ «2012» \\ Abstract
}

Vocabulary plays an important role in language and literacy development. However, little is known about vocabulary development in young children who need to learn an official or majority language, in addition to a minority language spoken at home. This dissertation concerned English (majority) and Armenian (minority) vocabulary development in young bilingual children. First, a parallel bilingual vocabulary test was developed, which included picture identification and picture naming tasks to assess listening (receptive) and speaking (expressive) vocabularies in the two languages. Then, the vocabulary test was used to compare bilingual vocabulary growth and examine individual differences in vocabulary performance.

Twenty Junior and twenty-two Senior Kindergarten bilingual children were tested twice on the vocabulary tasks over a six-month interval. Information was also obtained on environmental (parental education, Armenian/English language exposure) and child characteristics (nonverbal cognitive abilities, phonological short-term memory) related to vocabulary development, as potential predictors of individual vocabulary performances. 
Children showed growth over time in all four vocabulary tasks. However, less growth was observed in Armenian expressive than in Armenian receptive, English receptive, and English expressive vocabulary. Children had high exposure to Armenian at home; however, consistent with their exposure to English through peers, most were proficient in English vocabulary. The findings suggest a preference to use English and the influential role of English language exposure on higher proficiency in English vocabulary. These findings imply a potential risk for Armenian language maintenance, which warrants future studies.

English language exposure and phonological short-term memory were both unique predictors of English vocabulary, whereas Armenian language exposure was the only unique predictor of Armenian vocabulary. Therefore, providing additional minority language exposure might be the best way to enhance and maintain minority vocabulary and language.

This dissertation had three novel contributions. It provided a sound and replicable method to develop a bilingual vocabulary test in other minority languages. The vocabulary test made it possible to compare performance across minority and majority languages directly and confidently for the first time. Finally, the relative values of four different characteristics were examined in predicting individual differences in minority and majority vocabulary performances. 


\section{Acknowledgments}

I owe my deepest gratitude to all of the people who have provided me with their support, advice, and encouragement throughout the years leading to the completion of my studies. My appreciation is beyond words for my supervisor, Dr. Carla J. Johnson who has made my $\mathrm{PhD}$ journey a rewarding learning experience. I am tremendously grateful for her invaluable guidance and expertise, which have helped me in getting a deeper understanding of research, in general, and my subject of study, in particular. I am also indebted to my supervisory committee members Dr. Alice Eriks-Brophy, Dr. Esther Geva, and also to Dr. Luigi Girolametto who was on my committee for a short while. I am heartily grateful for their expert contributions to my work. Their critical comments provided me with a broader perspective of my study.

I am also grateful for having the opportunity to work in an environment surrounded by people whose welcoming approaches from the beginning made me feel at home while thousands of miles away from my home country. My sincere gratitude goes to the faculty, especially Carla J. Johnson and Alice Eriks-Brophy for their ever-present kindness and personal support, the staff, and my fellow-students in the department. I am also grateful to Joanne DeLuzio, Deryk Beal, Sophie Lafaille, Elizabeth Rochon, and Laura Laird for their willingness to share their knowledge along with some of their assessment tools needed for my study.

Through my study I came to know the Armenian community in Toronto, which was not only a great personal experience but also provided me with the best opportunity and circumstances to implement my study. I would like to extend my deepest gratitude to all of the individuals from the Armenian community who at different stages of my study provided their invaluable support and help. Bella Mousalou and Linda Erzroomi deserve special mention for their cooperation in the study. The schools have been extremely cooperative and facilitated the implementation of my study. Special thanks go to the school principals; Arpi Ajemian, Armen Martirossian, Hasmig Kourdian, and Mrs. Hopyan; the School Boards; and the kindergarten teachers, especially Mrs. Hasmig. My gratitude is endless to the parents and the children for their time and enthusiasm in participating in my study.

My parents and siblings have always been an integral part in my pursuit of studies, especially in moving to Canada for my PhD. I have always been encouraged and supported by their blessings 
for which I am grateful all my life. I am also grateful to my husband, Robert and our little daughter Talida for bearing with me throughout these years leading to the completion of my studies. I have been blessed by having the loving support and encouragement of Robert and the joy that Talida has brought into my life. The extreme joy that she experiences in learning every new word makes me more aware of the importance of language and especially home language.

I would also like to acknowledge the funding I have received throughout my $\mathrm{PhD}$ program from the University of Toronto, Department of Speech-Language Pathology. 


\section{Table of Contents}

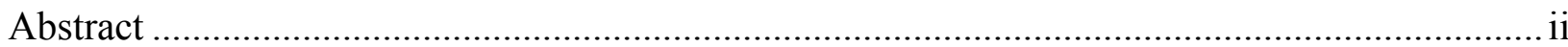

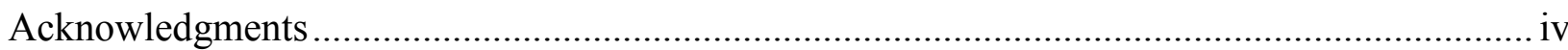

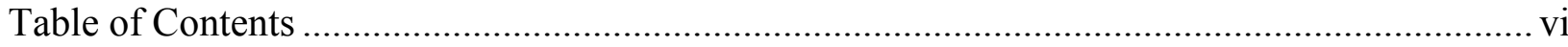

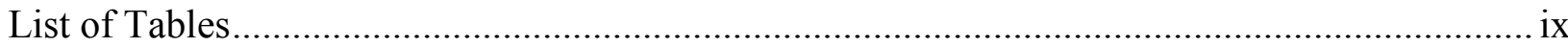

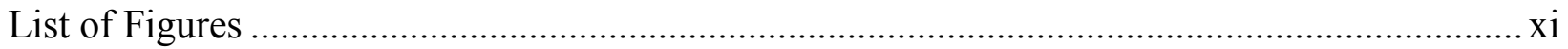

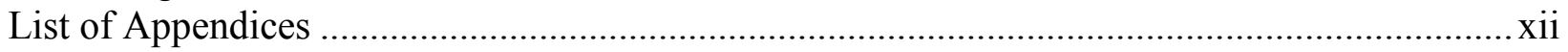

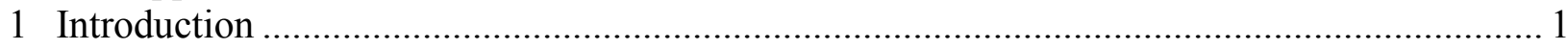

1.1 Overview of the Importance of Bilingualism and Bilingual Vocabulary ........................... 1

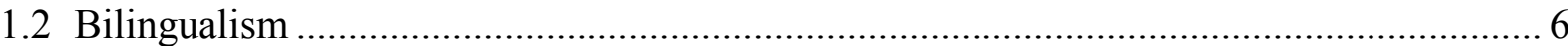

1.2.1 Definition and Classification of Bilingualism...................................................... 6

1.2.2 Historical Overview of Studies on Bilingualism ................................................ 7

1.2.3 Theoretical Perspectives on Bilingualism ………............................................ 7

1.2.3.1 Additive vs. Subtractive Bilingualism ................................................... 7

1.2.3.2 Language Dominance and Proficiency ………………………….............. 9

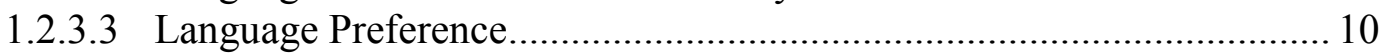

1.2.3.4 Language Maintenance ………………........................................... 10

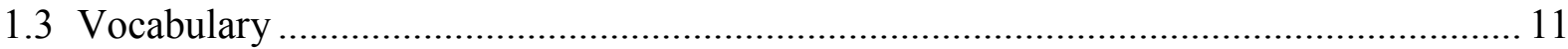

1.3.1 Theoretical Perspectives on Vocabulary Differences between Bilinguals and Monolinguals.................................................................................................. 12

1.3.1.1 Distributed Characteristics of Bilingual Vocabulary Knowledge .......... 13

1.3.1.2 Basic vs. Academic Vocabulary Skills in Bilinguals ............................. 15

1.3.2 Factors Related to Vocabulary Development........................................................ 15

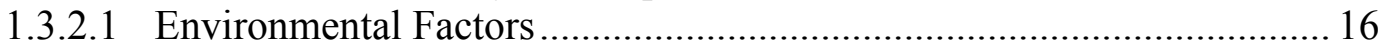

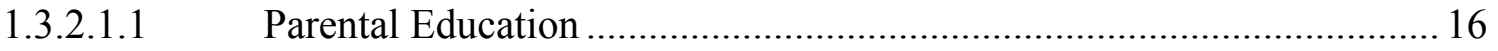

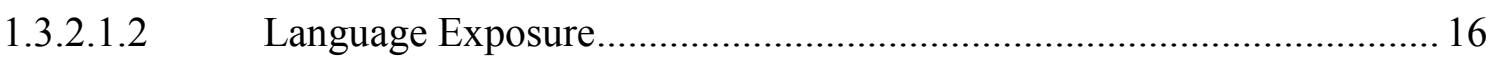

1.3.2.1.3 Language Value ............................................................................. 18

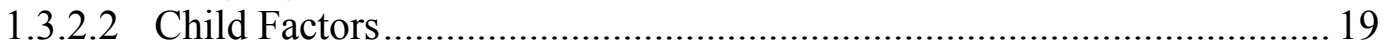

1.3.2.2.1 Nonverbal Cognitive Ability ……….............................................. 19

1.3.2.2.2 Phonological Short-Term Memory ........................................................ 19

1.4 Review of Vocabulary Studies in Young Bilinguals .....................................................2. 22

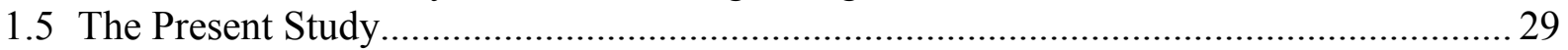

1.5.1 Why Armenian as the Minority Language? ........................................................ 30

1.5.1.1 Armenian Bilinguals in Canada ................................................................. 31

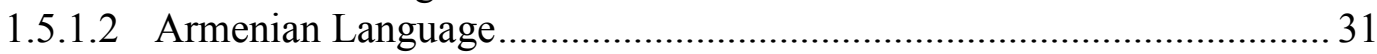

1.5.1.3 Armenian-English Bilingual Programs................................................... 32

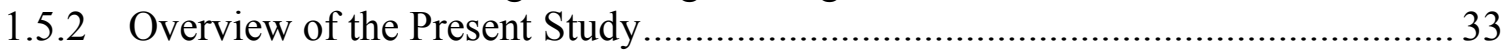

1.5.3 Objectives and Predictions of the Present Study ................................................... 34

1.5.4 Organization of the Present Dissertation............................................................... 35

2 Study 1: Development of a Bilingual Vocabulary Test.................................................................36

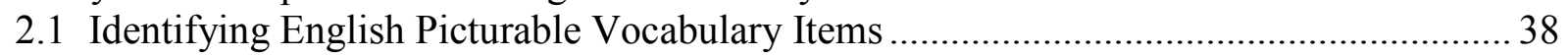

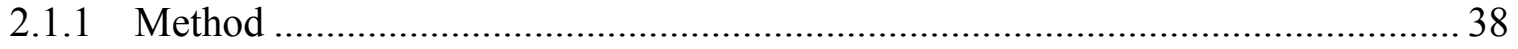

2.1.1.1 Vocabulary Items Appropriate for Young Children ............................... 38

2.1.1.2 Vocabulary Items Appropriate for Picture Identification and Picture

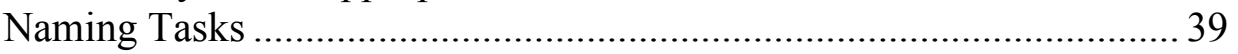

2.1.1.3 Vocabulary Items Appropriate for Armenian and English ..................... 40 


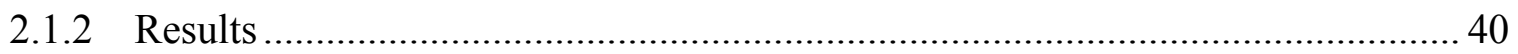

2.2 Obtaining Common Labels and Age of Acquisition Ratings.......................................... 40

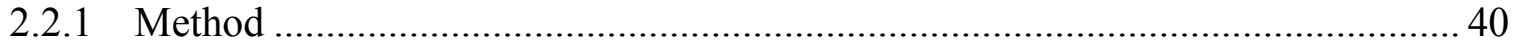

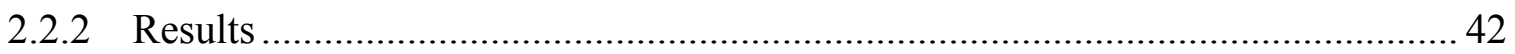

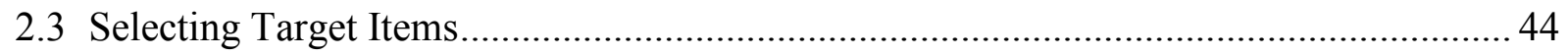

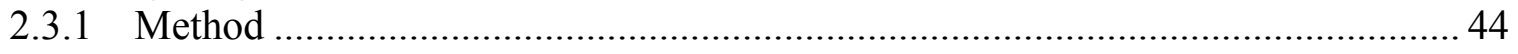

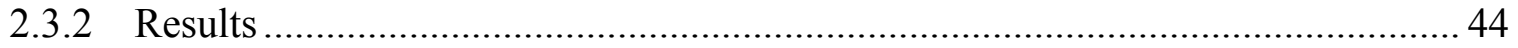

2.4 Developing Four Parallel and Independent Vocabulary Lists ......................................... 44

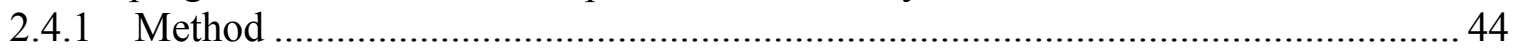

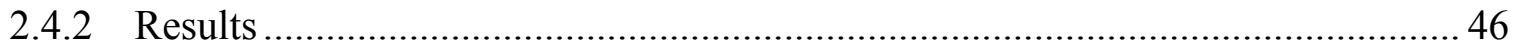

2.5 Selecting Foil Pictures for the Identification Tasks ...................................................... 48

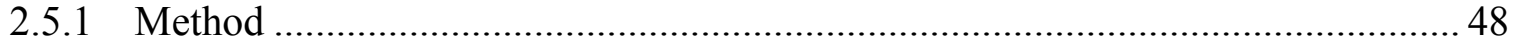

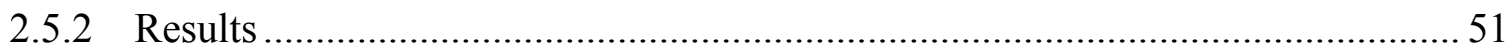

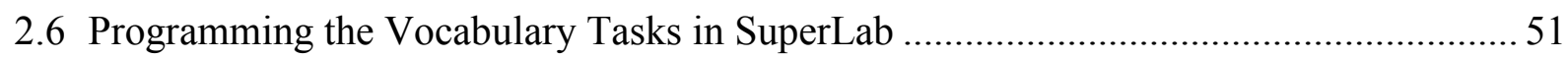

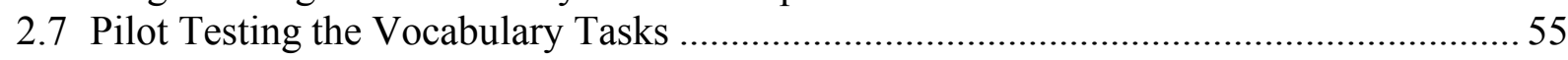

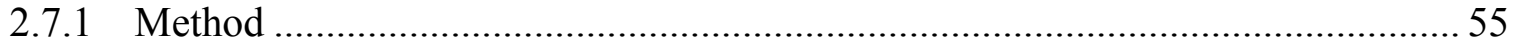

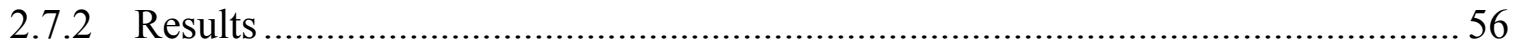

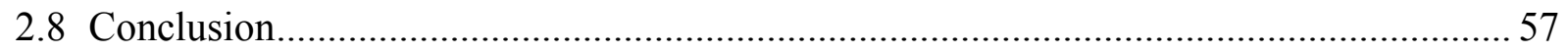

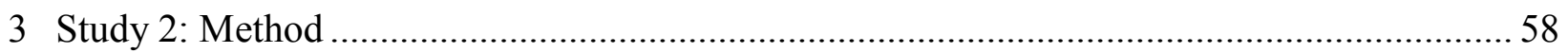

3.1 Sample Size and Power Analysis ........................................................................... 58

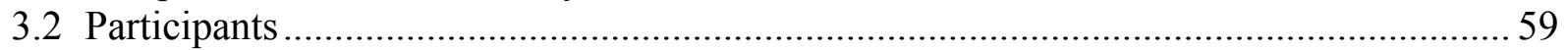

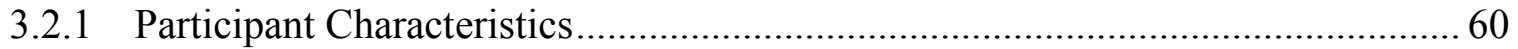

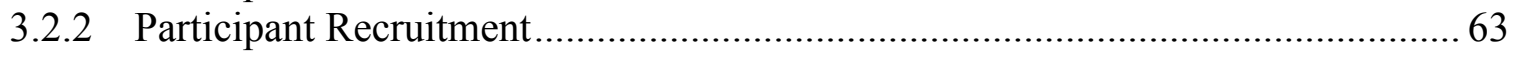

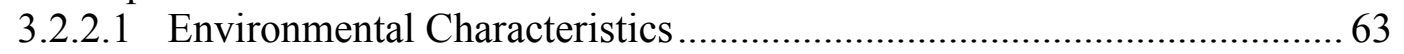

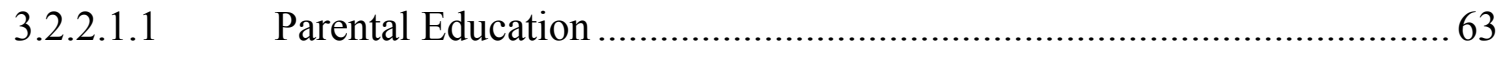

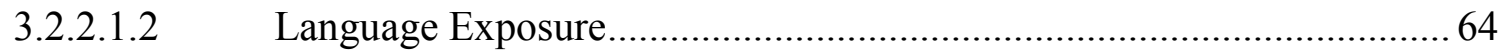

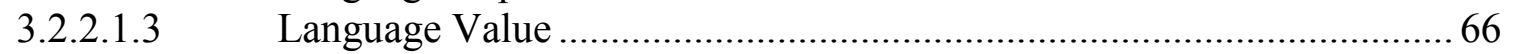

3.2.2.1.4 Additional Information on Family Characteristics ................................... 67

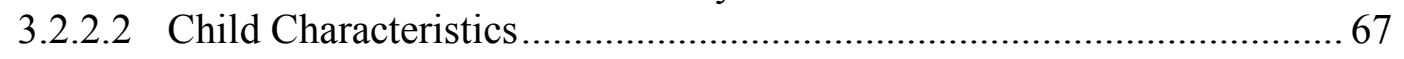

3.2.2.2.1 Nonverbal Cognitive Ability ................................................................. 67

3.2.2.2.2 Phonological Short-Term Memory ……….............................................. 69

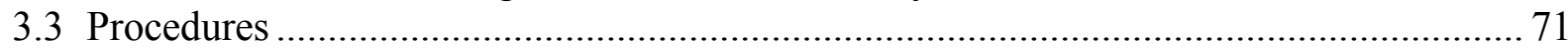

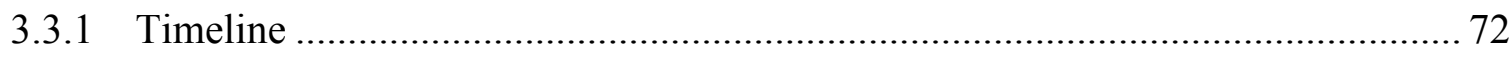

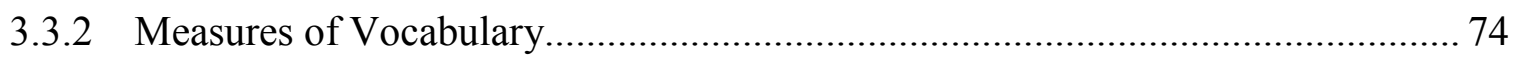

3.3.2.1 Settings and Apparatus ................................................................... 74

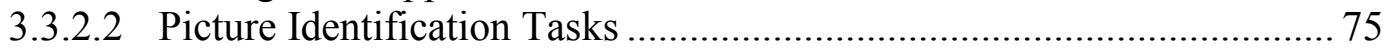

3.3.2.3 Picture Naming Tasks ........................................................................... 76

3.3.3 Additional Measures ..................................................................................... 78

3.3.3.1 Language Development …………………...................................... 78

3.3.3.2 Hearing Abilities ............................................................................ 79

3.3.4 Program Fidelity ................................................................................... 79

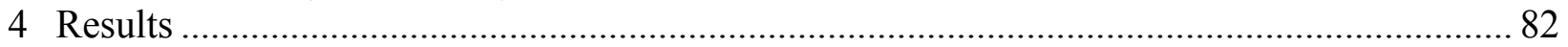

4.1 Preliminary Analysis on Possible Effects of List Order .................................................. 82

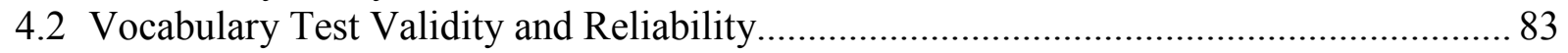

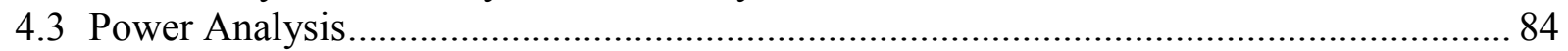

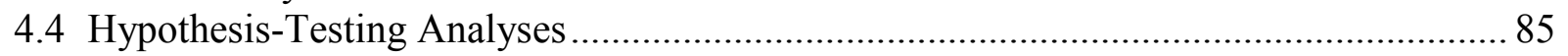

4.4.1 Group Differences in Bilingual Vocabulary Performance................................... 85

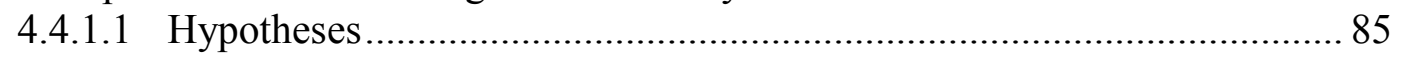


4.4.1.2 Descriptive Statistics on Vocabulary Performance ................................ 86

4.4.1.3 Mixed Analysis of Variance of Group Differences in Vocabulary

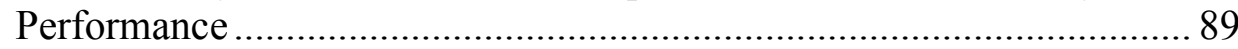

4.4.1.4 Supplementary Analyses of Group Differences in Vocabulary

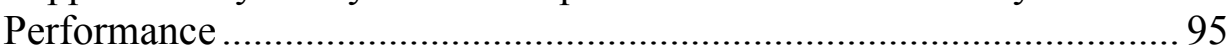

4.4.1.4.1 Mixed Analysis of Covariance …………................................................ 95

4.4.1.4.2 Relative Vocabulary Proficiency and Language Exposure ................... 100

4.4.2 Individual Differences in Vocabulary Performance................................................ 108

4.4.2.1 Potential Predictors of Armenian and English Vocabularies................. 108

4.4.2.2 The Relationships among Environmental and Child Predictors and Armenian and English Vocabularies .................................................. 109

4.4.2.3 Environmental and Child Predictors of Armenian Vocabulary .............. 111

4.4.2.4 Environmental and Child Predictors of English Vocabulary................. 114

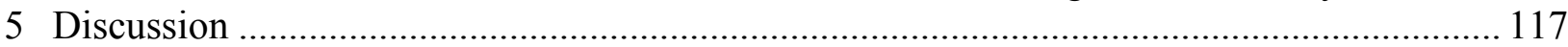

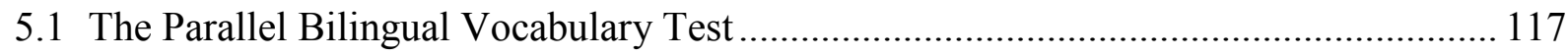

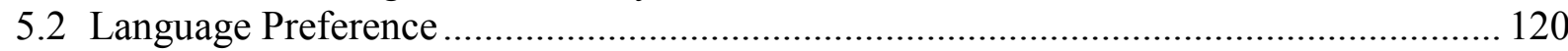

5.3 Language Exposure and Patterns of Vocabulary Proficiency ......................................... 126

5.4 Predictors of Individual Differences in Armenian and English Vocabulary

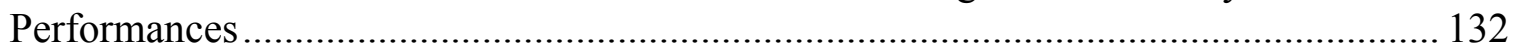

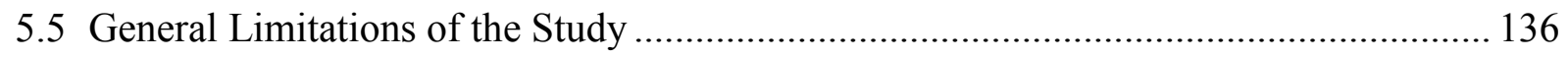

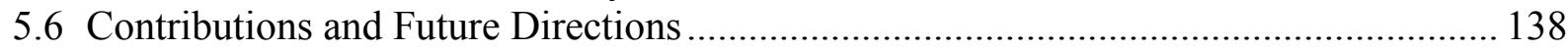

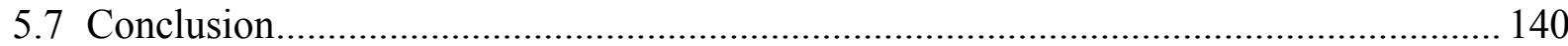

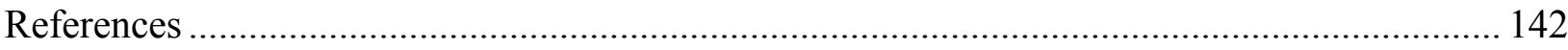

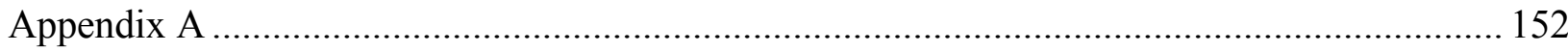

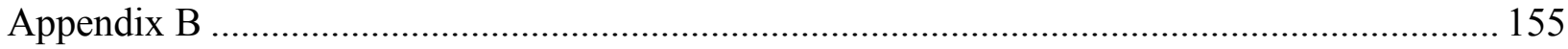

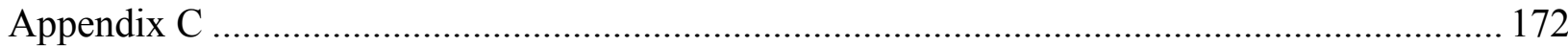

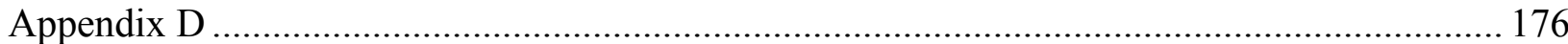

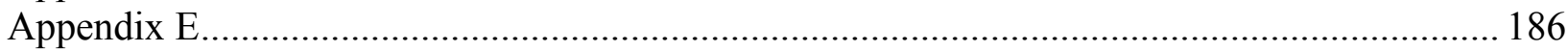

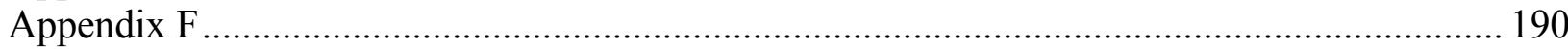

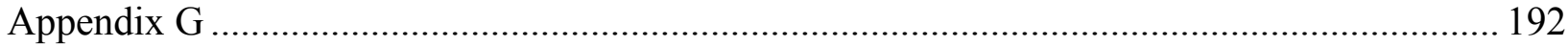

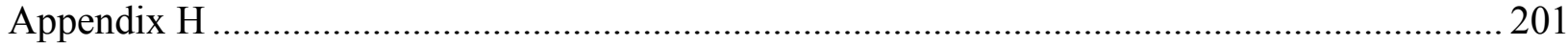

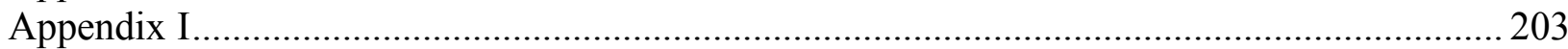

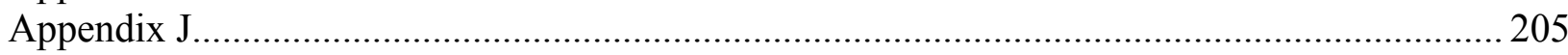




\section{List of Tables}

Table 1. Means (M) and Standard Deviations (SD) for Age of Acquisition (AoA) and Labelling in the Four Lists

Table 2. First Four Consecutive Target Items in Each of the Four Lists with Their Respective Foils.

Table 3. Means (M) and Standard Deviations (SD) for Age of Acquisition (AoA) Values in Years for the Targets and their Foils in the Four Lists and Results of Tests of Significance 52

Table 4. Means (M), Standard Deviations (SD), and Ranges for Participant Characteristics in Junior (JK) and Senior Kindergarten (SK) Grades, and Results of Tests of Significance....

Table 5. Timetable for Task Administration at Time 1 and Time 2

Table 6. Means (M) and Standard Deviations (SD) of Percent Correct Scores in Armenian and English Vocabulary Tasks in Junior (JK) and Senior Kindergarten (SK) Grades at Time 1 and

Time 2

Table 7. Mixed Analysis of Variance of Between- and Within-Subjects Variables on Vocabulary Task Performance 90

Table 8. Mixed Analysis of Covariance of Between- and Within-Subjects Variables on Vocabulary Task Performance, with English Language Exposure at Home as the Covariate .... 98

Table 9. Means (M) and Standard Deviations (SD) in Three Subgroups of Vocabulary Proficiency for Percent Correct Scores in Vocabulary Tasks, Percentages of Language Exposure and Language Use, Standard Score in Nonverbal IQ, and Percent Correct Score in PSTM .... 105

Table 10. Pearson Correlations among Vocabulary and the Predictors (Armenian in top panel; English in the bottom $[N=38]$ ). 110

Table 11. Summary of Hierarchical Multiple Regression Analyses for Environmental and Child Predictors of Armenian Vocabulary Performance $(N=38)$ 
Table 12. Summary of Hierarchical Multiple Regression Analyses for Environmental and Child Predictors of English Vocabulary Performance $(N=38)$....................................................... 115 


\section{List of Figures}

Figure 1. A sample of the picture naming task trial with 'star' as the target item. (From the World Wide Web at http://crl.ucsd.edu/ aszekely/ipnp/. Reprinted with permission.) .............. 53

Figure 2. A sample of the picture identification task trial with 'star' as the target item. (From the World Wide Web at http://crl.ucsd.edu/ aszekely/ipnp/. Reprinted with permission.)

Figure 3. Significant 3-way interaction of Language by Modality by Time, collapsed across Grade, with lines representing mean percent correct scores in Armenian (left panel) and English (right panel) picture identification and picture naming tasks at Time 1 and Time 2.

Figure 4. Significant 2-way interaction of Language by Grade, collapsed across Modality and Time, with bars representing mean percent correct scores in Armenian and English vocabulary tasks in Junior Kindergarten (JK) and Senior Kindergarten (SK) children.

Figure 5. Significant 4-way interaction of Language by Modality by Time by Grade with lines representing adjusted mean percent correct scores in Armenian (left panel) and English (right panel) picture identification (PI) and picture naming (PN) tasks in Junior Kindergarten (JK) and Senior Kindergarten (SK) grades.

Figure 6. Vocabulary growth in the three subgroups of Balanced, Armenian-Proficient, and English-Proficient bilinguals with lines representing mean percent correct scores in Armenian and English vocabulary tasks at Time 1 and Time 2 


\section{List of Appendices}

Appendix A Written Permissions for Using Pictures ............................................................. 152

Appendix B All Potential Vocabulary Items in Alphabetical Order........................................ 155

Appendix C Information Letter and Informed Consent Form for Teachers in Study 1;

Information Letter and Informed Consent Form for Parents in Study 1 ................................ 172

Appendix D Four Lists of Selected Target Vocabulary Items .................................................... 176

Appendix E Information Letter and Informed Consent Form for Parents of Children in Pilot

Study; Child Assent Script for Participants in Pilot Study...................................................... 186

Appendix F Home Language Use Questionnaire.................................................................... 190

Appendix G Information Letter and Informed Consent Form for Parents of Children in Study 2, Time 1; Information Letter and Informed Consent Form for Parents of Children in Study 2, Time 2; Child Assent Script for Participants in Study 2, Time 1- Day 1; Child Assent Script for Participants in Study 2, Time 1- Day 2; Child Assent Script for

Participants in Study 2, Time 2- Day 1; Child Assent Script for Participants in Study 2, Time 2- Day 2

Appendix H Hebrew Pseudo-word Phonological Short-Term Memory Task; Task

Administration Instructions 201

Appendix I Informed Consent Form for Teachers in Classroom Observation 203

Appendix J Classroom Observation Checklist; Instructions.................................................... 205 


\section{Introduction}

\subsection{Overview of the Importance of Bilingualism and Bilingual Vocabulary}

Bilingualism, according to Bhatia and Ritchie (2004), is a rule rather than an exception in today's world, and it will continue to be so in the future. Crystal (1997) estimated that two-thirds of the children throughout the world grow up in bilingual environments with exposure to more than one language. For many children, bilingualism is not a choice but rather a fact of life that is tied to circumstances present in family and community. Different circumstances give rise to bilingualism, such as being raised in a family with parents speaking different languages, or growing up in a country where the dominant and majority language is different from the minority language used at home by parents (Genesee, Paradis, \& Crago, 2004).

The fact that for many children bilingualism is not a choice emphasizes bilingual children's need to learn both languages. Language is a powerful tool through which individuals have access to resources available in the community (Louis \& Taylor, 2001). Minority-language bilinguals, whose home language differs from the majority language, belong to both minority and majority communities. By learning their home or minority language, these individuals have access to the knowledge shared by those speaking the same language, and by learning the majority language they have access to the educational and vocational resources available in the majority community. In addition, it is widely believed that the development of the home language plays a crucial role in the development of cultural and ethnic identity, membership in one's cultural community, and continued contact with family and community members (Feuerverger, 1991; McCardle, Kim, Grube, \& Randall, 1995; Miller \& Hoogstra, 1992; Wong Fillmore, 1991).

Vocabulary is an important aspect of language learning. The importance of vocabulary is emphasized in the role that it plays in the development of proficiency in communicating information through the use of linguistic forms (Paul, 2001). Receiving information through linguistic input also plays a significant role in language development. In fact, vocabulary skills

have an important role in the comprehension of input. For example, in her review, Altman (1995) showed the importance of the lexicon in sentence comprehension. Comprehension of input in 
turn enhances language acquisition, especially in children exposed to a language other than their home language (Gass \& Selinker, 1994).

Vocabulary is also related to the development of other aspects of language such as syntax (Fenson, Dale, Reznick, Bates, Thal, \& Pethick, 1994). Conboy and Thal (2006) showed that the grammatical abilities of 2-year-old bilinguals were more related to same-language vocabulary development than to maturation or general lexical-conceptual development, emphasizing the importance of vocabulary in the development of grammar. In addition, they found that an increase in Spanish and English sentence complexity was related to growth in same-language vocabulary.

Another important aspect of vocabulary is its relation to literacy development. The relationship between vocabulary and literacy has been established not only in monolingual children (Dickinson, McCabe, Anastasopoulos, Peisner-Feinberg, \& Poe, 2003) but also in children learning two languages. For example, in one study, the Spanish and English expressive vocabulary abilities of bilingual kindergarten children predicted their respective reading comprehension in the two languages in first grade (Lindsey, Manis, \& Bailey, 2003). In another study, Spanish and English receptive vocabulary abilities predicted letter-word identification abilities in both languages in first grade (Hammer, Lawrence, \& Miccio, 2007).

Obtaining information on typical vocabulary development in bilingual children has important practical implications. Developmental as well as educational expectations are built based on this information. These expectations are then used as measures for diagnosing developmental deviations and designing curriculum (McCardle \& Hoff, 2006). Delay or deviation in vocabulary development is one of the early indicators of language problems. By investigating typical vocabulary development, abnormal development can be better understood and predictions can be made based on early signs of language disorders (Bloom, 2000). Therefore, information on typical vocabulary development can be used in therapeutic settings for assessing bilingual children's vocabulary development and identifying possible developmental deficits (Goldstein, 2006).

Information on typical bilingual vocabulary development can also address many questions and concerns encountered by families of bilingual children from minority-language communities. These families want their children to learn both the home language and the majority language 
(Genesee et al., 2004). However, sometimes misinformed by negative perspectives on bilingualism, they may be concerned about possible negative effects that maintaining their home language might have on their bilingual children's success in learning the majority language (Barac \& Bialystok, 2011). Therefore, by having a better understanding of typical vocabulary development in bilingual children, many of these concerns can be addressed in a more realistic manner.

In addition to the practical and clinical implications discussed above, obtaining information on vocabulary development in young bilingual children has theoretical implications. It can inform us of the capacities of the children in learning two languages and the factors involved in this learning.

Research has provided a better understanding of vocabulary development, particularly in monolingual children (Bloom, 2000; Clark, 2003). However, very little is known about typical vocabulary development in young bilingual children who learn English in the presence of their home language, a minority language (Kohnert, Yim, Nett, Kan, \& Duran, 2005, McCardle \& Hoff, 2006). This limited knowledge may result in practical problems for those in charge of the educational needs of these children and also for those responsible for assessing and treating their potential speech and language problems. The sparse literature on typical vocabulary development of bilingual children increases the challenges involved in providing language services to these children (Kohnert et al., 2005). To compensate for the limited information on bilingual vocabulary acquisition, information on typical monolingual vocabulary development is often used in its place (Genesee et al., 2004). Generally, the use of monolingual norms for bilinguals may be misleading because it fails to capture the unique pattern of bilingual vocabulary development, which usually occurs in different contexts for the two languages. For example, according to Gathercole, Thomas, and Hughes (2008), a vocabulary measure based on monolingual norms may provide a skewed picture of the vocabulary knowledge of bilinguals if it does not consider the difference in the amount of input that bilingual children receive in their two languages.

The importance of vocabulary calls for more contributions to the literature on bilingual vocabulary development. The increasing population of minority-language children in the country (Statistics Canada, 2007) is yet another reality that adds to the necessity of minority-English 
bilingual studies. However, to date there are very few studies on the acquisition of any linguistic domain, especially vocabulary, in young children with typical development who learn English as well as their home language (Kohnert et al., 2005). One of the reasons for the scarcity of studies on this population might be the wide variability among different groups of bilinguals. Language acquisition occurs in a context and different contexts influence language learning in different ways that give rise to variability among bilinguals (Bialystok, 2007).

To address this variability, Gonzalez (2001) concluded that it is essential to have a multidimensional perspective on language studies involving minority-language bilingual children. In such a perspective the interactions between external or environmental factors, including socioeconomic and sociocultural circumstances, as well as internal child factors, such as biological and psychological attributes, are taken into consideration. According to Gonzalez, external or environmental factors include parents' educational level and the language used at home and internal factors include psychological-cognitive aspects of the child. Furthermore, Pearson (2007) suggested that the factors associated with language learning in bilinguals, such as language exposure, language status, and language support through schooling, differ from one language community to another. Therefore, to understand language development, especially vocabulary growth in bilingual children from different communities, measuring these variables would be useful in explaining individual differences and normal variation.

The present study aimed to investigate vocabulary growth over six months in a group of young bilingual children who were learning English in addition to their home language, a minority language. This study involved the comparison of vocabulary growth in the receptive and expressive modalities of the minority and English languages of these young bilingual children. The comparison of both modalities of the two languages may provide a more comprehensive picture of bilingual vocabulary growth because these two modalities tap into two different aspects of vocabulary knowledge (Nation, 2001). Receptive vocabulary reflects passive knowledge used in recognizing words heard or read and expressive vocabulary reflects active use of vocabulary in its oral or written form (Nation, 1990). Typically, the number of vocabulary items recognized in the receptive domain far exceeds the number of words used in the expressive domain; however, there is a strong correlation between the two modalities (see Tomasello, 2003 for a review; Webb, 2008). 
In this study, cross-modality and cross-language comparisons were made possible by using a researcher-developed parallel bilingual vocabulary test. This test consisted of four lists of vocabulary items equivalent in rated age of acquisition (AoA), an estimate of the age at which the vocabulary items were acquired. The lists were then counterbalanced across picture identification and picture naming tasks in the two languages for assessing performance in the receptive and expressive vocabularies of the bilinguals in both of their languages. The picture identification tasks measured receptive vocabulary and the picture naming tasks measured expressive vocabulary in their respective languages.

The development of the vocabulary test was the first attempt in minority-majority vocabulary studies, in general, and in Armenian-English bilinguals, in particular, to provide a measure based on rated AoA that could be used for comparative assessment of vocabulary performance in both the receptive and expressive modalities of the two languages. There are significant challenges involved in designing a vocabulary measure equally applicable to both modalities and languages of bilingual speakers. For example, vocabulary items may differ across languages not only in their rated AoA, but also in structural aspects such as phonological or morphological complexity and in the circumstances in which those items are acquired and used. These differences in turn can result in learning different vocabulary in the two languages, making it difficult to compare vocabulary development in the two languages of bilinguals. These challenges, as suggested by Thordardottir (2005) can be, to some extent, overcome by using normative data, which can provide a more valid measure for comparing children's performances relative to their age peers. However, the fact is that vocabulary development in most minority languages, including Armenian, has not yet been studied systematically enough to yield normative data for more reliable cross-language comparisons. While acknowledging these limitations of the use of a nonstandardized bilingual vocabulary measure, which is equivalent only on rated AoA of its constituent items, the development of the vocabulary measure used in this study was a preliminary attempt to provide a measure as comparable as possible for the two languages and modalities.

This study contributed to a better understanding of vocabulary growth in young bilingual children by providing information on the patterns of bilingual vocabulary growth and on the interaction of these patterns in the two languages of the bilinguals. This study also investigated 
individual differences in vocabulary by examining the predictive value of environmental and child characteristics in accounting for individual differences in vocabulary performance.

This chapter will introduce the study, first by providing a general overview of bilingualism and relevant studies. Then the introduction will focus on vocabulary research. Finally, before describing the present study, a review of previous studies on vocabulary in minority-language bilingual children will provide an overview of the relevant literature and its existing gaps.

\subsection{Bilingualism}

\subsubsection{Definition and Classification of Bilingualism}

Bilingualism, as defined by Grosjean (1982), refers to the use of two languages for communicative interaction. Bilingualism encompasses a wide range of subgroups, which are differentiated with respect to different factors. For example, Genesee et al. (2004) used two factors for classifying bilingualism. One of the factors was the timing or temporal sequence of the learning of the two languages. Individuals learning two languages from birth or from early ages in the preschool years are considered simultaneous bilinguals. These individuals are distinguished from sequential bilinguals, who learn an additional language after learning the first one, usually after three years of age.

The other factor proposed by Genesee et al. (2004) for classifying bilingualism is the ethnolinguistic community of the languages spoken by bilinguals. The majority-minority distinction is derived from this factor. A language from a majority community, as compared to a minority language, is widely used, highly valued, and receives institutional support from the government. For example, in Canada, English and French are majority or official languages, whereas the languages spoken at home by different ethno-linguistic minority communities, such as Chinese or Greek, are classified as minority or non-official languages. However, these languages are considered majority languages in their respective countries.

Canada represents a country with different groups of bilinguals, including those with a minority language as their home language. The ethno-cultural profile of Canada is moving towards multiethnicity and multi-culturalism (Statistics Canada, 2001). Currently, about 20\% of people in Canada speak a minority or non-official language as their mother tongue (Statistics Canada, 2007). In addition, over $27 \%$ of the population of Ontario and $49 \%$ of the population of the 
Greater Toronto Area (GTA) speak a non-official language, other than English or French, as their mother tongue (Statistics Canada, 2007). This highlights the existence of different communities of bilinguals from different ethnic and linguistic backgrounds. Children from these communities grow up with their home language as the minority language and English or French as the majority language.

\subsubsection{Historical Overview of Studies on Bilingualism}

Considering the fact that a considerable proportion of the world population is growing up bilingual, more studies are needed to focus on language acquisition in this population. So far, most studies have focused on the fundamental aspects of language acquisition, such as syntax, in monolingual children, especially during the first three years (Clark, 2003). It was estimated that only $2 \%$ of language studies were focused on bilingual acquisition (Bhatia \& Ritchie, 1999). To date there is an insufficient number of studies examining children acquiring two languages (McCardle \& Hoff, 2006). Those available are focused mainly on school-age children, leaving younger bilingual children understudied (Fernald, 2006).

The study of bilingual acquisition has a long but non-continuous history. The first scientific report of a bilingual child dates back to 1913 followed by a longitudinal case study by Werner Leopold of his two bilingual daughters published in the 1940s (as cited in Genesee, 2006). From then on there was a gradual increase in the number of bilingual studies until the 1980s, when bilingual research experienced a boost resulting in more studies on bilingual acquisition. According to Genesee (2006), the majority of these studies were on morpho-syntactic development, with limited studies examining the phonological and vocabulary domains.

\subsubsection{Theoretical Perspectives on Bilingualism}

\subsubsection{Additive vs. Subtractive Bilingualism}

Early studies on bilingualism with a focus on developmental capacities provided a negative picture of bilingual acquisition. For example, Macnamara (1966) reported that bilingual children showed lower levels of cognitive abilities as compared to monolinguals. It was suggested that the acquisition of two languages had a negative influence on the cognitive development of bilingual children. Based on findings from such studies, Macnamara introduced an influential theory on the limited linguistic and cognitive capacities of bilinguals. According to this theory, as 
proficiency in one language increases, proficiency in the other language decreases. This theory encouraged a pessimistic view, which considered bilingualism problematic. Such thinking still prevails among many lay people and professionals (Genesee et al., 2004).

A major breakthrough in bilingual research happened in the 1960s when Lambert and his colleagues introduced a new and more optimistic perspective on bilingualism. They found that earlier studies were flawed as a result of methodological limitations. The major cause for concern was that the monolingual and bilingual children compared in earlier studies came from different environments in terms of socioeconomic status (SES), with bilinguals representing lower SES than monolinguals and with different levels of proficiency in each of their two languages. To address these limitations, Peal and Lambert (1962) compared the language performance of English-French proficient bilinguals with that of their monolingual peers from the same SES background in Quebec. They found that the bilinguals performed better than their monolingual peers on measures of verbal intelligence and on nonverbal tests that involved concept-formation or symbolic flexibility.

Following this positive perspective in bilingual research, Lambert (1977) identified two different circumstances in which minority-language bilingual children might learn the majority language. These have been referred to as subtractive vs. additive bilingual environments. In subtractive bilingualism, which occurs in a subtractive environment, the majority language is acquired at the expense of the minority language. In this case, the main focus for minority-language children is to learn the majority language, which is considered more important, and to forget their minority language. Previously, this approach was considered the best way to learn the majority language, because it was thought to free up the language learning capacity necessary for learning the majority language (Volterra \& Taeschner, 1978). In contrast, in an additive environment, which nurtures additive bilingualism, continuous support is provided for the minority language while children experience learning the majority language. Therefore, in additive bilingualism, both languages are given similar opportunities to develop and thrive.

Early pessimistic views on bilingualism have also been questioned as a result of evidence from other studies, some of which have focused on developmental milestones in bilingual acquisition. These studies showed that the age of acquisition of critical milestones in bilinguals did not differ from those of their monolingual peers. For example, similar ages of onset for babbling were 
found in bilingual and monolingual infants (Oller, Eilers, Urbano, \& Cobo-Lewis, 1997). In addition, the ages of acquisition of first words and first two-word combinations in FrenchEnglish bilingual children were found to be similar to monolingual norms (Petitto, Katerelos, Levy, Gauna, Tetreault, \& Ferraro, 2001). Studies have also documented the cognitive advantages of bilingual children over their monolingual peers in tasks such as metalinguistic awareness (see Bialystok, 2001 for a review). Finally, other studies have found that bilingual children show vocabulary growth in both their languages (Rodriguez, Diaz, Duran, \& Espinosa, 1995; Winsler, Diaz, Espinosa, \& Rodriguez, 1999). These findings indicate that, contrary to pessimistic views on bilingualism, proficient acquisition of two languages is within the capabilities of children.

\subsubsection{Language Dominance and Proficiency}

Language dominance or relative proficiency is an important phenomenon related to minoritymajority distinctions and patterns of language use among bilinguals. Language dominance usually occurs in bilingual children with minority-majority combinations of languages and characterizes the pattern of use of their languages (Genesee et al., 2004). As discussed by Genesee et al., the two languages acquired usually do not develop equally, and it is usually unrealistic to expect balanced bilingualism. For example, it has been shown that younger bilingual children are usually proficient in both of their languages, but when they become older they shift dominance to the majority language, which is usually the language of schooling and formal education (Kohnert \& Bates, 2002; Kohnert, Bates, \& Hernandez, 1999). In these studies, dominance or proficiency in the two languages of bilinguals was decided based on the relative performance of five age groups $(5-7,8-10,11-13,14-16$, and 18-22 years) in their minority and majority receptive and expressive vocabularies.

Language exposure is an important correlate of language proficiency or dominance. In their study on 2-year-old Spanish-English bilingual children, Pearson, Fernandez, Lewedeg, and Oller (1997) showed that the vocabulary knowledge of children in each language was closely related to the amount of exposure or input received in that language. The measures of vocabulary and exposure were based on estimates provided by parents of the percentage of words produced in each language and of the percentage of language exposure in each language, respectively. 


\subsubsection{Language Preference}

Bilingual children may prefer to use one language over another regardless of their dominance or proficiency in that language. Research has shown that bilingual children as young as two years of age adapt their language choice to their adult interlocutors (Genesee, Nicoladis, \& Paradis, 1995). Language choice or the preference to use one language over the other can be related to factors other than language dominance or proficiency. In other words, a bilingual child might choose to use the majority language more often than the minority language, regardless of his/her dominance or proficiency in one or the other. For example, the language choice of high-school Spanish-English bilingual students showed a shift towards English outside the home. Interestingly enough, this shift in language choice was unrelated to the Spanish proficiency of the bilinguals; rather, it was related to their language attitudes and preferences (Hakuta \& D’Andrea, 1992). The same finding was observed in younger Spanish-English bilinguals (Orellana, 1994).

Peers play an important role in the language choice of bilingual children because bilinguals usually prefer to use the majority language of the dominant culture with their siblings and friends (Garcia, 1983). School is one of the situations where bilingual children have considerable contact with peers. Peer interactions and the use of the majority language for instruction can have a major influence on the patterns of language use and language choice in bilinguals (Orellana, 1994). Overall, these findings suggest that minority-language bilingual children might choose to use the majority language, not because of their lack of proficiency in their minority language but because of peer pressure or sheer preference to use the majority language. This would be especially true with peers who do not speak the minority language.

\subsubsection{Language Maintenance}

The choice and preference of bilingual children to use the majority language has implications for minority language maintenance. As discussed above, if bilingual children use the majority language under the influence of peers and schooling, then one should be concerned about the negative consequences of this preference on the use and maintenance or attrition and loss of the minority language. In fact, parents have voiced their concern over the loss of the home language in their children who have attended English preschools (Wong Fillmore, 1991). Based on a largescale interview survey of parents from a minority-language community, Wong Fillmore reported 
a language shift in more than half the families. This shift reflected a change in the pattern of language use at home by children attending preschools, with English taking over the minority language. According to Wong Fillmore, this language shift might be related to the powerful forces of assimilation encountered by minority-language children upon entering school, resulting in minority-language attrition and loss.

Researchers have long been interested in identifying the factors related to language maintenance or loss in minority-language populations in a dominant majority culture context. In one of the early studies using interview data, Hesbacher and Fishman (1964) identified institutional support, such as minority-language instruction, as an important factor in the language maintenance of two immigrant groups in the United States. In a more recent study, Guardado (2002), using interview data from Hispanic families in Vancouver, showed the importance of cultural identity in minority language maintenance. Cultural identity was defined as attachment to the Hispanic culture.

Despite the strong influence of English or other majority languages on the language choice of minority-language bilinguals, there are factors that may enhance minority language maintenance. For example, Hakuta and D'Andrea (1992) found that maintenance of proficiency in the minority language in high-school Spanish-English bilingual students was basically related to the use of the language at home by the bilinguals and their parents or other adults. This proficiency was not predicted by language choice or preference outside the home. In addition, in the same study, based on response latencies in Spanish naming and recognition tasks, the authors concluded that the gradual attrition of the Spanish language was more a difficulty in proficient retrieval of words from memory rather than actual language loss.

To conclude, the practical message regarding bilingualism and the relevant research findings and theoretical perspectives is that bilingualism is not a phenomenon in isolation. To have a better understanding of bilingual acquisition, one might consider the language abilities of the bilinguals as well as the circumstances surrounding their bilingualism.

\subsection{Vocabulary}

Vocabulary has drawn the attention of an immense amount of studies in monolingual acquisition research. Preschool children are one of the age groups that have received the attention of many 
vocabulary studies (Bloom, 2000). According to Bloom this group is of interest because it represents a midpoint between 1-year-old novices who have just started learning vocabulary and the older children who are experts in vocabulary learning.

The preschool years are marked by significant growth in different aspects of language, especially in vocabulary (Paul, 2001). In fact, one of the most prominent features of 2- to 5-year-old English-speaking children is their rapid growth in vocabulary (Bloom, 2000). After the acquisition of first words, which occurs at about 12 months of age, there is a rapid increase in vocabulary called the 'vocabulary spurt', which starts at about 16 to 19 months of age, by which time children have already acquired about 50 words (Goldfield \& Reznick, 1990). By 24 months of age, children with typical development reach an average expressive vocabulary of 300 words (Fenson et al., 1994). From then on, children continue acquiring new vocabulary on a daily basis (Bloom, 2000) so that by the age of 18 expressive vocabulary reaches a level of approximately 60,000 words (Aitchinson, 1994).

The acquisition of vocabulary encompasses two separate modalities, each with a different rate of development. Generally, vocabulary is classified into two distinct groups of receptive or passive vocabulary and expressive or active vocabulary. According to Nation (1990), passive (receptive) vocabulary knowledge refers to the ability to recognize a word and recall its meaning when encountered, whereas active (expressive) vocabulary knowledge refers to the ability to use or write a word when needed. Receptive and expressive vocabulary are interrelated in that, typically, comprehension precedes production and children understand words before producing them (Clark, 1993). In addition, due to a difference in the nature of these two vocabulary modalities, learning the expressive mode is considered to be more difficult than learning the receptive mode (Nation, 1990). As a result, usually receptive vocabulary is larger than expressive vocabulary in monolinguals as well as bilinguals (e.g., Aitchinson, 1994; Fan, 2000; Webb, 2008). In general, to have a better understanding of acquisition and growth in vocabulary, it is important to examine both receptive and expressive aspects concurrently.

\subsubsection{Theoretical Perspectives on Vocabulary Differences between Bilinguals and Monolinguals}

Among the main questions raised in bilingual acquisition research is whether bilingual children show the same developmental stages as monolinguals in different domains of language. 
Generally speaking, typical language development entails the acquisition of milestones that are usually acquired in a sequential manner. For example, generally children start saying single words before combining words into sentences at a later time. Many research studies on language acquisition in bilinguals have been conducted on syntax. However, research on vocabulary development of bilinguals is more limited than in other domains (Genesee, 2006).

Research on the comparison of language acquisition in bilinguals and monolinguals has resulted in a different picture for vocabulary as compared to other aspects of language such as syntax. For example, studies on syntax have unanimously found that bilingual children show the same developmental milestones in the syntax of their two languages with comparable levels of proficiency in both. For example, French-English bilingual children showed the same milestones and levels of proficiency in the acquisition of functional categories and negation in both of their languages (e.g., Paradis \& Genesee, 1996; 1997). Yet, studies on vocabulary have documented smaller vocabulary sizes in each of the two languages of young bilingual children in standardized tests as compared to their monolingual peers (Nicoladis \& Genesee, 1996; Umbel, Pearson, Fernandez, \& Oller, 1992). The following theoretical perspectives may provide some explanations for the observed differences between monolingual versus bilingual vocabulary sizes.

\subsubsection{Distributed Characteristics of Bilingual Vocabulary Knowledge}

To explain the variable performance of bilingual children on standardized vocabulary tests, Oller and Pearson (2002) proposed the hypothesis of distributed characteristics of vocabulary knowledge in bilinguals. According to this hypothesis, bilingual vocabulary is partially distributed across two languages. Bilingual children usually know words for some concepts in one language but not in the other language. Children might know both translation equivalents for some concepts but not for others. Therefore, based on the distributed characteristics hypothesis, the low vocabulary scores in bilinguals might not necessarily suggest that they are poor vocabulary learners.

Presumably, the distributed nature of bilingual vocabulary knowledge might reflect the fact that bilingual children learn and use the vocabularies of their two languages in different contexts and under various circumstances. Vocabulary learning in children occurs through interactions with the world around them while being exposed to a spoken language (Genesee et al., 2004). In fact, 
what makes a significant impact on vocabulary acquisition is the context in which the interactions occur and the amount of language exposure that a child receives during these interactions (Genesee, 2006). Genesee further argues that there are some fundamental differences between monolinguals and bilinguals in the context of vocabulary learning and amount of language exposure, which add to the variability in vocabulary learning between bilinguals and monolinguals. Bilingual children learn each of their two languages in different contexts, because they are usually exposed to and use each language with different people and in different circumstances. In addition, the extent to which bilingual children are exposed to each of their two languages separately is less than the exposure that the monolinguals receive in their single language. Therefore, as a result of the differences in context and exposure, a bilingual child might end up having different vocabulary items and vocabulary sizes in their two languages. However, Pearson, Fernandez, and Oller $(1993$; 1995) showed that the vocabulary size of the Spanish-English bilinguals was equivalent to that of their monolingual peers once they obtained a total conceptual vocabulary size for bilingual children. To obtain the size of the total conceptual vocabulary they combined the vocabulary items known in each of the two languages and then excluded any translation equivalents. The combined result represented the concepts known across the two languages, regardless of the labels known for these concepts in either or both languages.

To further address the question of presumed lower vocabulary performance in bilinguals, Bialystok, Luk, Peets, and Yang (2010) performed a large-scale analysis of data on English receptive vocabulary combined from different studies. The data included mean standard scores of 1,738 bilingual children between the ages of three to 10 years on the Peabody Picture Vocabulary Test-III (PPVT-III; Dunn \& Dunn, 1997). The results showed a significantly lower mean standard score in bilinguals compared to monolinguals. However, an item analysis of the vocabulary showed that the bilingual/monolingual difference was mainly confined to vocabulary items related to home life, whereas school-related vocabulary was more comparable in the two groups. They concluded that bilingual children were not disadvantaged in terms of their academic vocabulary in English. The difference between bilinguals and monolinguals was mainly in home life vocabulary knowledge in English. However, bilinguals knew those words in their home language. The findings might well explain the smaller size of vocabulary in each of 
the two languages of bilingual children as compared to their monolingual peers, when the two vocabularies are considered separately.

\subsubsection{Basic vs. Academic Vocabulary Skills in Bilinguals}

Cummins (1979) introduced two different types of majority-language vocabulary skills that require different time frames in order for bilingual children to gain competence. The distinction was made between basic interpersonal communicative skills (BICS) and more advanced cognitive academic language proficiency (CALP). According to Cummins (1981), minoritylanguage bilingual children gain a functional level of interpersonal conversational fluency within about two years of first exposure to the majority language, whereas it takes at least five years to gain grade-appropriate academic proficiency in the majority language.

Therefore, Cummins (1979) suggested that the basic versus academic distinction should be taken into consideration in assessing bilingual children's academic performance. The degree of conversational fluency (BICS) observed in bilingual children may not necessarily mean that they have attained adequate academic language proficiency. On the other hand, lack of proficiency in the latter (CALP) might interfere with the academic achievement of bilingual children.

\subsubsection{Factors Related to Vocabulary Development}

Normal variation in vocabulary development is related to a variety of factors, which range from external environmental factors such as language exposure to internal child-related factors such as cognitive abilities (Hoff, 2005). Many factors related to vocabulary variability in young bilingual children are similar to those documented in monolinguals. In her review article, Pearson (2007) explored five factors: input or language exposure, family language use, language status, access to literacy, and community support including schooling. She concluded that all of these factors influence vocabulary learning in bilingual children.

In what follows, some of the factors reported to play a significant role in normal variability in the vocabularies of young monolingual as well as bilingual children will be described. Two groups of factors are described, following the environmental/child dichotomy mentioned above. The environmental factors include parental education, language exposure, and language value. The child-related factors include nonverbal cognitive ability and phonological short-term memory. 


\subsubsection{Environmental Factors}

\subsection{Parental Education}

Parental education level is an environmental factor considered to be of significant importance in vocabulary learning. In a large-scale study, Fenson et al. (1994) analyzed the productive vocabularies of 1,803 monolingual babies and toddlers. Information on vocabulary was obtained using vocabulary inventories completed by parents. Children of parents with higher education showed higher vocabularies than children of parents with lower education. Parents with higher education have also been shown to talk more to their children than parents with lower education (Hart \& Risley, 1995; Hoff-Ginsberg, 1994). Another study documented that the vocabulary used by parents with higher education was more sophisticated and versatile than that of parents with lower education, which was simpler and more restricted (Hoff \& Naigles, 2002). Finally, 5year-old minority-language bilingual children of mothers with higher education were found to have higher rates of growth in receptive and expressive English vocabulary than those of mothers with lower education (Golberg, Paradis, \& Crago, 2008).

Parental education is a frequently used general indicator of SES (Galobardes, Lynch, \& Smith, 2007) because parental education correlates with family SES. For example, Cobo-Lewis, Pearson, Eilers, and Umbel (2002) reported that the SES of Hispanic families in Miami was related to their educational background. The parents with high SES had total years of education ranging between 14.1 and 15.6 years, whereas the parents with low SES had total years of education ranging between 10.5 to 12.7 years.

\subsection{Language Exposure}

Language exposure is another significant variable in the vocabulary development of monolingual as well as bilingual children. Through interactions with others, especially parents, the child is exposed to language and gets experience in using language. Language exposure has typically been measured using parental report (see for example, Gutierrez-Clellen \& Kreiter, 2003; Parra, Hoff, \& Core, 2011). Logically parental report is considered to be a reliable source for providing information on language use and proficiency of children, because the parent observes the child in so many different situations over long periods of time (Thordardottir \& Weismer, 1996).

Researchers have also examined the question of the reliability of parental report in determining bilingual children's language use. For example, Gutierrez-Clellen and Kreiter (2003) found 
significant correlations between parental ratings of bilingual children's language use in Spanish and English and the actual use of grammatical utterances in each language as measured by spontaneous narrative samples using a wordless picture book. In addition, they found significant correlations between parental and teacher ratings of children's language use. Similarly, the reliability of parental report was also examined in determining bilingual children's use and proficiency in their two languages by investigating the relationships between parental ratings and child language performance. Significant correlations were observed between parental ratings of their children's linguistic abilities such as grammar (Gutierrez-Clellen \& Kreiter, 2003) or vocabulary (Sheng, Lu, \& Kan, 2011) and the children's actual grammatical or vocabulary performances.

The literature on monolingual children provides evidence of the relationship between language exposure and vocabulary. For example, Hurtado, Marchman, and Fernald (2008) showed that 18month-old Spanish children who heard more maternal speech had a greater increase in their expressive vocabulary at 24 months as measured by parental report, compared to children whose mothers talked less with them. Maternal speech during a play session with the child was recorded and analyzed for the frequency of use of utterances and different types of words. A longitudinal study that followed vocabulary development in 14-month-old monolingual English-speaking children for one year, found that children who received more child-directed speech with more diverse vocabulary showed higher rates of vocabulary development than those receiving less child-directed speech (Huttenlocher, Haight, Bryk, Seltzer, \& Lyons, 1991). In another study, the richness of vocabulary and syntactic complexity of mothers' utterances was found to predict productive vocabulary in their children (Hoff \& Naigles, 2002).

Studies have also investigated the relationship between language exposure and language development, including vocabulary in bilinguals. For example, Pearson et al. (1997) found strong correlations between the extent of language exposure and words known by 8- to 30-month-old Spanish-English bilinguals in each language. The extent of exposure that 2-year-old bilingual children received in their minority language, as estimated by parents, was correlated $(r=.82)$ with the percentage of their expressive vocabulary knowledge in that language. To measure expressive vocabulary, a standardized vocabulary inventory was filled out by parents. Along similar lines, David and Wei (2008) investigated the relationship between language exposure and vocabulary in bilingual children. This study was carried out on French-English 1- to 3-year-old 
bilingual children living in France or in the United Kingdom. The children came from families where parents had different native languages and each spoke his/her language to the child, i.e., they followed one-parent-one-language practice. Data on vocabulary were obtained from parental report on child vocabulary at 23 and 30 months of age. Language exposure was measured as the reported percentage of input received in each language by the child. This study found a significant correlation between the language exposure index in each language and the size of vocabulary in that language. In other words, children exposed to more French or English showed bigger vocabulary size in that language.

More recent studies have also investigated the relationship between language exposure and vocabulary in bilinguals. For example, in their study on Moroccan-Dutch and Turkish-Dutch bilinguals, Scheele, Leseman, and Mayo (2010) documented significant relationships between parental reports of exposure that 3-year-old Moroccan-Dutch bilingual children received in Tarifit-Berber (minority language) through storytelling $(r=.41)$ and conversations $(r=.39)$ and receptive vocabulary performance in that language measured by a picture identification vocabulary test. For the Turkish-Dutch group these correlations were .33 and .45 , respectively. Finally, Parra et al. (2011) showed a correlation of .72 between parental reports of English language exposure and English expressive vocabulary in 2-year-old Spanish-English bilinguals. These findings showed significant correlations between language exposure and vocabulary in different groups of bilinguals.

Language exposure can also occur through other activities such as television viewing. Studies have documented that children can learn new English vocabulary while watching television programs, especially educational ones. For example, positive effects of watching television programs such as "Sesame Street" were observed on the vocabulary development of 3- to 5-yearold children (Rice, Huston, Truglio, \& Wright, 1990).

\subsection{Language Value}

The value that a minority-language community has for its home language might have a differential role in the development of the home language among its young members, especially once they start learning the majority language. As discussed in Section 1.2.3.1, Lambert (1981) distinguished between additive and subtractive bilingualism based on the value and use of each language. In additive bilingualism both minority and majority languages are valued and 
supported equally, which presumably leads to comparable development in both. On the contrary, in subtractive bilingualism one language is valued and used more than the other, which may lead to attrition in the less-valued language. For example, in a study with Hispanic families in Canada, parents believed that the lack of a positive attitude in parents toward their home language was one of the factors having an adverse influence on the minority-language maintenance of their children (Guardado, 2002).

\subsubsection{Child Factors}

\subsection{Nonverbal Cognitive Ability}

Nonverbal cognitive ability is a child-related factor that may influence vocabulary learning in both monolinguals and bilinguals. Based on a review of studies on vocabulary, Bloom (2001) argued that cognitive abilities are related to vocabulary learning. A longitudinal study investigated the role of nonverbal cognitive abilities in vocabulary development in 4-year-old children with and without intellectual disabilities (Van der Schuit, Segers, Van Balkom, \& Verhoeven, 2011). Nonverbal cognitive abilities predicted vocabulary in children with typical development $(\beta=.56)$ at age four but not at age five. In addition, when nonverbal cognitive abilities were taken into account, children with intellectual disabilities no longer showed significant differences in vocabulary from the children with typical development. This finding indicated that vocabulary in children with intellectual disabilities was on pace with their intellectual level.

There is also evidence of a relationship between nonverbal cognitive abilities and vocabulary in bilinguals. For example, a correlation was found between home language vocabulary development and general cognitive abilities in Turkish children who were learning Dutch as the majority language (Leseman, 2000). General cognitive abilities were measured as a composite score including nonverbal intelligence and logo-mathematical concept knowledge. Overall, the relationship between nonverbal cognitive abilities and vocabulary warrants the inclusion of this variable in studies examining individual differences in vocabulary.

\subsection{Phonological Short-Term Memory}

Phonological short-term memory (PSTM) is thought to be a sub-system within working memory and a specialized mechanism for retention of verbal information for a short time (Baddeley, 
2003). Several studies have shown a relationship between PSTM and expressive language development in children (e.g., Adams, Bourke, \& Willis, 1999; Adams \& Gathercole, 1996; 2000). Based on a review of studies of vocabulary learning, Baddeley, Gathercole, and Papagno (1998) suggested that PSTM helps with remembering familiar words and learning new ones. Familiar words are remembered by retaining sound sequences in phonological memory and new words are learned by storing unfamiliar sound patterns in phonological memory. PSTM has been shown to be a good predictor of second language learning ability in children (Service, 1992).

One of the paradigms used to measure PSTM is nonword repetition. This is the ability to hear and repeat back pseudo-words, which are not real words but are constructed based on the phonology of a specific language. This ability, assumed to depend on PSTM, predicted the acquisition of vocabulary in monolingual children (Gathercole \& Baddeley, 1989; Gathercole, Hitch, Service, \& Martin, 1997). There was also a close link between nonword repetition and the ability to learn new words in monolinguals (Gathercole, 2006) and vocabulary growth in bilinguals across time (Farnia \& Geva, 2011). From a theoretical perspective, it was originally hypothesized that PSTM was an independent cognitive capacity responsible for processing and storing phonological information (Baddeley et al., 1998). However, more recent arguments, based on a review of relevant studies, suggest that PSTM might be affected by external factors such as language input received through exposure (MacDonald \& Christiansen, 2002). In examining the relationship between vocabulary and PSTM, two distinct groups of nonword repetition tasks have been used. Depending on the purpose of the study, some researchers used a nonword repetition task with stimuli similar to the target language to investigate the question of the effect of language exposure and familiarity on PSTM task performance (e.g., Parra et al., 2011). However, Farnia and Geva (2011) used a task with stimuli different from the target language, in order to allow performance in PSTM tasks to be captured independently from language familiarity.

As mentioned earlier, factors in addition to PSTM have been found to be related to vocabulary development. Therefore some researchers have investigated the contribution of PSTM to vocabulary performance in the presence of other factors. For example, Adams et al. (1999) found that PSTM was related to vocabulary knowledge in 4- and 5-year-old English-speaking children. PSTM, as a composite score, was measured by English nonword repetition and memory span for words and digits. Vocabulary knowledge was measured by a picture identification test. In 
addition to the PSTM tasks, other predictor variables included in this study were age, nonverbal ability, and visual memory. PSTM predicted an additional $9.9 \%$ of variance in vocabulary, after age and nonverbal ability were entered. In a study by Parra et al. (2011), two sets of PSTM tasks, one including English-like stimuli and the other Spanish-like stimuli, were used to assess the relationships among language exposure, PSTM, and expressive vocabulary in each language of 2-year-old bilingual children. Language exposure was measured as percentages of exposure to English and Spanish as reported by primary caregivers. PSTM for English-like stimuli explained $35 \%$ of the variance in English expressive vocabulary and language exposure accounted for an additional $25 \%$ of the variance in vocabulary after removing the effects of PSTM. In addition, PSTM for Spanish-like stimuli explained 12\% of variance in Spanish expressive vocabulary and language exposure accounted for an additional $31 \%$ of variance in vocabulary after removing the effects of PSTM. These findings indicate the significant contribution of PSTM to Spanish and English vocabularies as well as the unique contribution of language exposure to Spanish and English vocabularies over and above the variance accounted for by PSTM in each language.

There is also evidence that children with better PSTM have better language performance. Adams and Gathercole (2000) investigated the relationship between individual differences in spoken language acquisition, PSTM (as measured by an English nonword repetition task), and other aspects of verbal memory in 4-year-old monolingual children. Two groups of children were identified based on the results of the repetition task, one with good nonword repetition performance and the other with poor performance. They were matched for nonverbal abilities as an indicator of general cognitive abilities. Spontaneous speech samples were taken from children in both groups while they described pictures showing scenes of different actions. Children with better PSTM abilities used more words and longer utterances in their speech. Finally, Service (1992) showed that PSTM (as measured by an English nonword repetition task) was related to English vocabulary performance of 9-year-old Finnish children learning English as a foreign language. English proficiency was measured by a subjective rating of the English performance of the children by their teachers and by objective English test scores. In summary, this literature suggests the contribution of PSTM to language acquisition in general and to vocabulary in particular. 


\subsection{Review of Vocabulary Studies in Young Bilinguals}

To date there is sparse literature on vocabulary development in both languages of young bilingual children who learn English as the majority language in addition to their home language, a minority language. The focus of most studies has been on school-age children (e.g., Kohnert, 2002; Kohnert \& Bates, 2002; Kohnert, et al., 1999) or on young children with a majority language other than English (e.g., Leseman, 2000; Scheele et al., 2010). This leaves less than a handful of studies on young, 3- to 5-year-old bilingual children with English as their majority language and their home language as the minority language. These studies, which will be the focus of the following review, include Rodriguez et al. (1995), Winsler et al. (1999), Kan and Kohnert (2005), and Sheng et al. (2011).

In the first of these studies, Rodriguez, et al. (1995) compared the vocabulary growth of a group of young children $(n=30)$ attending Spanish-English bilingual preschools in the United States, referred to hereafter as the preschool group, and a comparison group $(n=20)$ of Spanishspeaking children not attending preschool. All the children were in the age range of three to four years from families of poverty-level incomes. These children were living in a community with high support for the use of Spanish outside the home or school. For example, many stores in the community conducted their business in Spanish.

The preschool was on a full-day basis where children were exposed to a variable proportion of Spanish/English, which ranged from 30/70 to 70/30 with a modal proportion of 50/50, through informal instruction by a bilingual teacher. The children were tested twice. The first testing was done when the children were in the preschool for two months, the second, six months later. The children were tested on Spanish and English receptive and expressive vocabulary using different measures for the receptive and expressive modalities of the two languages. The Peabody Picture Vocabulary Test-Revised (PPVT-R; Dunn \& Dunn, 1981) measured English receptive vocabulary and the Spanish version of the PPVT (TVIP; Dunn, Padilla, Lugo, \& Dunn, 1986) measured Spanish receptive vocabulary. The lexical subtest of the Language Assessment Scales (LAS; De Avila \& Duncan, 1981) was used to measure expressive vocabulary in both Spanish and English using picture naming tasks. The use of different measures for the receptive modality of the two languages could make it difficult to perform direct comparisons of the two languages involving receptive vocabulary. 
This study, to my knowledge, was the first one using objective measures for assessing receptive and expressive vocabulary in the minority and majority languages of young children attending bilingual preschools. Objective vocabulary measures are derived from data on vocabulary performance of children and are more accurate representations of vocabulary skills in children than subjective measures, which are based on adult ratings of children's vocabulary performance (Morrison, Chappell, \& Ellis, 1997). The use of a longitudinal design and a comparison group made it possible to observe and compare developmental changes across time and groups. In addition, classroom observation provided information on the proportion of use of each language.

The preschool and comparison groups showed different rates of growth in one of the modalities of English vocabulary and they showed growth in both modalities of Spanish and English. There was a significant group by time interaction in English expressive vocabulary, which indicated a higher rate of English expressive vocabulary growth in the preschool group compared to the comparison group. Therefore, attending the preschool was related to a higher rate of growth in English expressive vocabulary. In addition, the two groups showed significant growth in Spanish and English receptive vocabularies over time. The groups also showed significant growth in Spanish and English expressive vocabularies. Finally, the preschool group showed an advantage over the comparison group only in English expressive vocabulary, but there were no group differences in Spanish expressive, Spanish receptive, and English receptive vocabularies.

The findings of higher performance and a higher rate of growth in English expressive vocabulary in the preschool group were predictable because they were presumably experiencing a higher rate of systematic English language exposure when compared with children staying at home. Nevertheless, there was an unexpected finding of similar performance of both groups in Spanish receptive and expressive vocabularies and especially in their English receptive vocabulary. The latter might be related to the English language exposure received by the children who stayed at home. However, no information was provided on the specifics of the use of English at home in the two groups.

While acknowledging the above-mentioned contributions of this study, two of its main limitations are discussed. First, there was a lack of information on some participant characteristics, such as general development and English language exposure at home that could act as sources of variability in vocabulary performance between and within the preschool and 
comparison groups. Second, the use of different measures for the two languages as well as for the receptive modalities of the two languages, made it impossible to compare confidently performance across languages and modalities and to examine possible language by modality interactions.

Winsler et al. (1999) investigated the long-term effects of a bilingual preschool program on the Spanish and English vocabulary development of young bilingual children in the United States in two related studies. The first study was a replication and the second was a follow-up of $82 \%$ of the original participants in Rodriguez et al.'s (1995) study. The preschool group in the follow-up study continued to attend the bilingual program for the second year and the comparison group continued to stay at home and not attend any formal program. Following the same procedures and using the same language measures as in the original study, Winsler et al. (1999) tested the participants after one year.

The replication study provided the same results as those reported in the original study, but the follow-up study provided both similar and different results from the original. Similar to the original study, the follow-up study showed significant growth in English and Spanish receptive and expressive vocabularies for both groups over the course of two years. Contrary to the findings in the original study, the follow-up study showed no significant differences between the two groups in their English expressive vocabulary or in their rate of growth in English. These findings were unexpected, as one would expect higher performance in English for children attending a bilingual preschool for the second year who were logically getting more exposure to English than children staying at home. These unexpected results might suggest the existence of other potential confounding variables. The most probable one might be the amount of English language exposure received by the children who were not attending a formal preschool program. Perhaps these children were somehow receiving as much English language exposure as their peers attending the preschool. However, the study did not provide enough information regarding this variable. As in the Rodriguez et al.'s (1995) study, limited information on participant characteristics makes it difficult to determine factors that may be influencing vocabulary development.

The minority language in the two studies reviewed so far was Spanish and the majority language was English. These two languages share similarities at the vocabulary level where there are a 
high number of cognate pairs, i.e., words with the same origin, shared by the two languages (August, Carlo, Dressler, \& Snow, 2005). Thus, studying a different combination of minorityEnglish languages, with few similarities shared between the two languages, would be desirable. To my knowledge, only two studies with young children have used different combinations of minority-English languages. These studies have focused on Asian languages, one study on Hmong-English and the other on Mandarin-English bilinguals.

In the first study of an Asian minority language, Kan and Kohnert (2005) investigated bilingual vocabulary performance in Hmong-speaking children learning English as the majority language in bilingual preschools in the United States. Unlike the Spanish community studied previously, which was well established with high support for Spanish outside the home, the Hmong population contained recent immigrants. The primary home language was Hmong and children were reportedly exposed to English only after entering educational programs. The children attended the bilingual program, which was set up for low-income families, two or three days a week. The program used both languages but in unequal proportions and for different purposes. Hmong was used for classroom management, whereas English was used for instructional purposes. This suggested disproportionate exposure to the two languages in school. In addition, the community that the participants came from was quite different from those of previous studies. As mentioned earlier, the Spanish-English children were from communities with extensive use and support of Spanish at home and school, whereas the use of Hmong by the children from the Hmong community was presumably more restricted to home.

Two age groups of children attending bilingual preschools were tested on their receptive and expressive vocabulary abilities in Hmong and English using researcher-developed picture identification and picture naming tasks. The receptive and expressive tasks contained different items, but the same tasks were used in both languages, making it possible to make comparisons across the two languages but not across the modalities. The older group $(n=9, M$ age $=5 ; 0$ years) was in the program for an average of 16 months, whereas the younger group $(n=10, M$ age $=3 ; 11$ years) attended for an average of nine months. Contrary to previous studies, using the same tasks for both languages in this study made it possible to examine differences in language performance. It also provided the opportunity to investigate conceptual vocabulary. The results of single-language performances and their comparisons are discussed below. 
Overall, the older group had higher performance levels than the younger group in vocabulary tasks. In addition, a significant language by group interaction indicated that the older group showed significantly higher performance in English compared to Hmong, whereas the younger group showed similar performances in Hmong and English. Higher English performance in the older group might be related to their longer experience in the program and, ultimately, higher exposure to English. Higher performance was observed in receptive vocabulary compared to expressive vocabulary in Hmong, but similar performance levels were observed in the receptive and expressive vocabularies of English. However, these observations cannot be interpreted clearly because of the use of different sets of vocabulary items in receptive and expressive tasks.

In this study, the higher performance of the older group in English compared to Hmong might well be explained by more systematic exposure to English. However, no information was provided on the language exposure that each group received outside school. This information could possibly explain differences in the vocabulary performance in each language. Furthermore, because of the cross-sectional design of the study, growth over time could not be investigated. In addition, caution should be used in making any developmental inferences, because the two age groups were independent, and any difference in their vocabulary performance might be attributed to within-group as well as between-group variability. Finally, this study used a researcherdeveloped measure for assessing receptive and expressive vocabulary performances. The measure consisted of picture identification and picture naming tasks. The tasks were the same for both languages, but two different sets of vocabulary items were used for the receptive and expressive modalities. Confident inferences cannot be made on comparisons involving modality, because no information was provided on whether the tasks for the two modalities were equally difficult.

In the second study of an Asian minority language, Sheng et al. (2011) investigated bilingual vocabulary performance in Mandarin-English bilinguals in the United States. Similar to Kan and Kohnert's (2005) study, Mandarin was mainly used at home, but unlike Hmong, Mandarin was not supported in the school. Thirty five children participated in the study. Eighteen children with a mean age of 4;4 years and 17 children with a mean age of 7;2 years formed the younger and older groups, respectively. With the exception of two children, the participants came from families where both parents were Mandarin-speakers. According to parental report, children received English language exposure mainly after attending daycare or preschool. The two groups 
were comparable in maternal education, Mandarin exposure, Mandarin proficiency as rated by parents, and age of English language exposure.

Similar to Kan and Kohnert's (1995) study, a researcher-developed vocabulary test was used to measure receptive and expressive vocabulary performances of the two age groups. This study also investigated conceptual vocabulary, however, only the information regarding singlelanguage performance and their comparisons will be presented here. The vocabulary test consisted of picture identification and picture naming tasks with different items for each task. However, the same tasks were used for Mandarin and English. No information was available on whether the two tasks were comparable in terms of difficulty of the items. Therefore, as in the previous study, confident comparisons can only be made between the two languages and not the two modalities.

The study reported four interactions. These interactions were: age by language, age by modality, language by modality, and age by language by modality. However, the inferences drawn from the interactions involving modality might not be accurate, because it was not shown whether the two tasks were equivalent in terms of difficulty. Therefore, the focus here will be only on the first interaction. The age by language interaction indicated a higher performance in English in the older group compared to the younger group, but similar performance in Mandarin in the two groups. The older group had been in school longer than the younger group and thus had received more exposure to English. This condition might well explain the group differences in language performance.

There were also significant main effects. The older group showed higher performance than the younger group. Performance in English was higher than performance in Mandarin. Higher performance in the older group compared to the younger group might be indicative of developmental trends in vocabulary. Experiencing extensive English language exposure at school might explain higher performance in English as compared to Mandarin.

The study also investigated language input and language output in Mandarin and English at home as predictors of vocabulary performance (picture identification and picture naming combined) in the two languages, respectively. Language input was defined as the parental estimate of the number of hours during the week that the child spent hearing the language at home. Language output was defined as the parental estimate of the number of hours during a 
week that the child spent speaking the language at home. These estimates were then converted to percentages and used as measures of language input and output. Based on the results of two stepwise regression analyses, Mandarin input accounted for $48.7 \%$ of the variance in Mandarin performance and Mandarin output accounted for an additional $6.2 \%$ of the variance. However, age was not a significant unique predictor of Mandarin vocabulary after the other two variables were entered. Age, an index of cumulative language experience, accounted for $47.5 \%$ of the variance in English vocabulary performance and English output accounted for an additional $12.3 \%$ of the variance. However, English input was not a significant unique predictor of vocabulary performance after the other two variables were entered. These regressions suggested that different factors contributed to Mandarin and English vocabulary. Both Mandarin language input and output made unique contributions to Mandarin vocabulary performance, whereas English output and not English input predicted English vocabulary performance.

In conclusion, belonging to the same minority-language community does not necessarily mean that all the members are experiencing the same kind and extent of bilingualism. The participants in the above studies were described to be primarily using the minority language at home and receiving English language exposure after attending preschool at about three years of age. This description would generally suggest that the participants were experiencing successive bilingualism. However, this conclusion might not be accurate, as different conditions may give rise to differences in the extent of successive bilingualism between the groups and among the children in each group.

In fact, it is common sense to consider the existence of a wide range of variability among these children in their bilingualism, from simultaneous acquisition of both languages to successive acquisition for those who truly have limited early exposure to the majority language. Different conditions might contribute to this variability. For example, children are often exposed to the majority language from early ages even before entering school, either because of inter-language marriages, where parents speak both minority and majority languages, or because of social relationships with neighbours or friends who speak the majority language. Early exposure to the majority language might even occur for families who use the minority language as the primary language of interaction at home. This might be the case in Rodriguez et al.'s (1995) and Winsler et al.'s (1999) studies, where the group staying at home showed the same level of proficiency in English receptive vocabulary as the group attending the preschool program. Equal proficiency in 
the two groups might be indicative of equal exposure to English; however, as mentioned earlier, these studies did not provide information on language exposure at home. In general, it is expected that children born into minority-language families might be exposed to varying amounts of minority and English languages from early ages.

Overall, the studies on vocabulary development in young minority-English bilingual children have made several important contributions, yet many gaps in the literature still remain to be addressed. These studies provided longitudinal as well as cross-sectional descriptive information on group performances in receptive and expressive vocabulary of the minority and English languages using objective measures. Based on the information provided by these studies we know that bilingual children from minority languages show growth in the receptive and expressive vocabularies of their two languages, but we do not know how the growth in the two modalities of their two languages compares and what cognitive and contextual factors make unique contributions to vocabulary development in each language. Although in previous studies objective measures were used to assess vocabulary in both languages, these measures were not always comparable across modalities and languages. Therefore direct comparisons and, specifically, interactions of language and modality could not be examined. Finally, the participants in three of the four studies described above were attending bilingual preschool programs with both the minority language and English being used as the languages of instruction. However, both languages were used by bilingual teachers and there was a variable range of use of each language. In one of the studies the minority language was only used for classroom management and English was the language of instruction. Even though the children were attending bilingual programs, the dissimilar use of the languages might have added another source of variability in vocabulary performance. In addition, except for the study by Sheng et al. (2011), the other studies did not address individual differences in vocabulary performance or provide information on language exposure at home, which could act as a predictor of vocabulary performance.

\subsection{The Present Study}

In an attempt to address the limitations of the previous studies, the present study (1) included a parallel bilingual vocabulary test with four equivalent lists of vocabulary items to compare crosslanguage and cross-modality differences, (2) investigated vocabulary growth over time in young 
bilingual children from an ethno-linguistic minority community in Canada using the parallel bilingual vocabulary test constructed for the study, and (3) measured language exposure and other possible predictors of individual differences in vocabulary. The language pair of interest in this study included the typologically different languages of English, the majority language, and Armenian, which is a minority language spoken in Canada by the Armenian ethno-linguistic community.

\subsubsection{Why Armenian as the Minority Language?}

The Armenian minority language was chosen for this study for the following reasons. This study is the first of its kind on Armenian-English bilingual children from Armenian ethno-linguistic community. To my knowledge, there are no previous studies comparing any aspect of language development, such as semantics, phonology, or syntax, in the two languages of young ArmenianEnglish bilinguals. Unlike Spanish, the language studied in two of the previous studies, Armenian shares few similarities with English. Therefore, studying this combination of minorityEnglish languages may provide new insights into vocabulary development in another minoritylanguage community. The Armenian community differs from the communities of previous studies in several aspects. Compared with the Spanish community in the United States, Armenians represent a very small community in Canada. Yet, despite a comparatively small community, Armenians have maintained their language after twelve decades of living in this country. Although Armenian is assumed to be the primary home language, a wide range of variability might be observed in Armenian and English use at home among the members of this community, especially children. The long establishment of the community in Canada, interlanguage marriages, and social relationships with English-speakers may suggest possible sources of exposure to English from early ages. Furthermore, the Armenian community, unlike Spanish, does not provide support for Armenian outside home, school, church, or community centres. However, Armenians are well-established and, in pursuit of Armenian language maintenance, they have established bilingual schools in the GTA, where this study was implemented, as well as in other parts of Canada such as Montreal. The GTA schools offer balanced bilingual programs for kindergarten children with the provision of equal educational conditions for both Armenian and English. This provides a unique opportunity for investigating vocabulary development in children attending a program where both languages receive equal institutional support. This kind of program, compared with an English-only monolingual program, has been 
shown to be beneficial for both minority and majority language development (Barnett, Yarosz, Thomas, Jung, \& Blanco, 2007; Duran, Roseth, \& Hoffman, 2010). Finally, the researcher is a member of this community, which gives her the attributes as well as the motivation to conduct a study on young members of the community.

\subsubsection{Armenian Bilinguals in Canada}

Armenians form one of the ethno-linguistic minority communities in North America, including Canada. Armenian is the language of this community. Armenians began settling in Canada in the 1880s (Kaprielian-Churchill, 2005). Currently there are over 30,000 first-language speakers of Armenian in Canada (Statistics Canada, 2010). It seems that Armenians value their language highly, because they continue to use their language even after twelve decades of settlement in this country. Another indication of the value ascribed to the language might be the establishment of bilingual educational settings geared towards teaching Armenian language and literacy alongside English.

First-language speakers of Armenian use Armenian most often at home (Statistics Canada, 2010); therefore, it is assumed that children from the Armenian community usually learn Armenian as their home language. However, this does not exclude the possibility of children being exposed to other languages at home, especially English, from early ages. Inter-cultural marriages between English- and Armenian-speakers might be one of the conditions in which children get exposure to and learn both languages from early ages from their parents. The official status of English is yet another condition providing early exposure to English. Armenian children might be exposed to English from early ages through watching television or communicating with English-speaking neighbors, peers, or older siblings.

\subsubsection{Armenian Language}

The Armenian language belongs to the Indo-European language family, however it is an independent branch (Malmkjær, 2002). Although Armenian and English both belong to the same language family, they are typologically different. For example, the normal word order in English is subject-verb-object (SVO), whereas in Armenian it is SOV. Armenian word order is more flexible than English. In addition, Armenian is morphologically an agglutinating language, meaning that word ending affixation is used for expressing a wide range of grammatical 
meanings such as plural or past tense (Hagopian, 2005). Furthermore, nouns and pronouns in Armenian undergo declension, i.e., they take different case markers, which are affixed to their endings, to fulfill different syntactic functions (Sakayan, 2000). The case markers are usually one-syllable. The cases in Armenian are nominative, accusative, genitive, dative, ablative, instrumental, and locative (Hagopian, 2005). For example, to indicate the locative case in Armenian, the suffix /um/ is added to the end of the nominative case of a noun. Therefore, the declension system in Armenian is very extensive compared to many other languages, including English. There are a few cases of declension in English. For example, nouns in English decline to reflect their grammatical number, e.g., book/books, or a small number of English pronouns decline to show their relationship to a verb or preposition, e.g., he/him. Finally, in terms of phonology, Armenian has six vowel sounds and 31 consonants. The main difference between Armenian consonant sounds and those in many other languages, including English, is that Armenian has voiced aspirated plosives and affricatives, in addition to the voiced and voiceless pairs present in other languages.

Armenian has two dialects, i.e., Western Armenian and Eastern Armenian. Both dialects are spoken in Canada and generally in North America. However, to my knowledge, official information is not available on the exact number and distribution of speakers of each dialect in North America. These dialects use the same Armenian alphabet, and almost the same vocabulary, but have slight differences in grammar and pronunciation. Thus, they are mutually understood by speakers of each dialect (Andonian, 1966).

\subsubsection{Armenian-English Bilingual Programs}

There are two Armenian private day schools in the GTA. The fact that these schools are private suggests that children attending them might be from families with high SES. One of the schools provides bilingual programs from kindergarten through the elementary grades; the other provides such programs through high school. However, no notable differences are observed between the two schools and their bilingual programs. The programs focus on the development of the abilities to listen, speak, read, and write in Armenian and English (Armenian General Benevolent Union Toronto, 2006). Armenian instruction focuses on Armenian language and literature, history, and religion. All of the academic subjects are taught only in English. 
At the time of the study, the kindergarten children in these two schools were attending separate Armenian and English classes taught by separate teachers. The programs were conducted with a half-day in Armenian and a half-day in English. During the first half of the school year, half the classes had Armenian in the morning and English in the afternoon and the other half of classes had Armenian in the afternoon and English in the morning. During the second half of the school year this schedule was reversed. The half-and-half ratio of Armenian to English programming was only sustained in both Junior Kindergarten (JK) and Senior Kindergarten (SK). After that, only a few hours per week were allocated to Armenian language instruction. No further information was available on the exact hours of Armenian instruction in each grade level past kindergarten. As mentioned earlier, the Armenian and English kindergarten classes were taught by separate teachers. Based on observation, the teachers, all female, were fluent speakers of the language they instructed. However, no data were obtained on the demographics of the teachers.

\subsubsection{Overview of the Present Study}

The primary objective of this dissertation was to investigate vocabulary growth in young bilingual children from the Armenian ethno-linguistic minority community. In a first study, a parallel bilingual vocabulary test was developed to measure Armenian and English receptive and expressive vocabulary. This test consisted of four vocabulary lists, equivalent in rated AoA, which were counterbalanced across four vocabulary tasks: (1) Armenian picture identification, (2) Armenian picture naming, (3) English picture identification, and (4) English picture naming. The picture identification tasks were used in assessing receptive vocabulary and the picture naming tasks were used in assessing expressive vocabulary. In a second study, participants were recruited from JK and SK grades in Armenian bilingual schools in the GTA. Participants from both grades were tested twice over a six-month interval on their Armenian and English receptive and expressive vocabularies using the researcher-developed parallel bilingual vocabulary test.

In addition to investigating group differences and growth over time, individual differences in Armenian and English vocabulary performance were examined. For this purpose, the roles of two groups of factors in vocabulary performance were examined. Information was obtained on various environmental and child characteristics using a researcher-developed parental questionnaire or direct assessments. These characteristics were explored as potential predictors of Armenian and English vocabulary performances. 
Finally, the classrooms were observed to verify the fidelity of the programs in providing a balanced pattern of use and instruction of both languages. The focus of these observations was on the frequencies of use of English in the Armenian classes and of Armenian in the English classes.

\subsubsection{Objectives and Predictions of the Present Study}

This study of bilingual vocabulary pursued two objectives. The first objective was to examine developmental trends in two groups of JK and SK bilingual children in Armenian and English receptive and expressive vocabularies. The second objective was to examine individual differences in Armenian and English vocabulary performances.

The first objective involved investigating differences in Armenian and English receptive and expressive vocabularies by comparing picture identification and picture naming task performances at two times of testing over a six-month interval in two groups of JK and SK children attending Armenian-English bilingual programs. Therefore, vocabulary task performance was analyzed as a function of Grade, i.e., JK and SK; Time, i.e., Time 1 and Time 2; Modality, i.e., Picture Identification and Picture Naming; and Language, i.e., Armenian and English. The second objective involved examining individual differences by identifying the best predictors of vocabulary performance. The study investigated the contributions of environmental and child characteristics to Armenian and English vocabulary performances.

The primary predictions of interest were:

(1) children in the SK would show higher vocabulary performance than children in the JK;

(2) vocabulary performance at Time 2 would be higher than vocabulary performance at Time 1;

(3) performance on picture identification would be higher than performance on picture naming;

(4) performance on Armenian vocabulary would be higher than performance on English vocabulary;

(5) the rate of growth in English vocabulary would be higher than the rate of growth in Armenian vocabulary; and

(6) each category of environmental and child characteristics would separately predict vocabulary performance in Armenian and English. 
The first three predictions were based on general trends in vocabulary development observed in research on monolingual as well as bilingual children. Because this was a first study of Armenian-English vocabulary development, it was thought to be important to replicate these well-known phenomena. Predictions 4 and 5 were novel and based on the assumption that the majority of children participating in this study would be from families where Armenian was the primary language spoken at home. However, upon entering the bilingual program with formal and considerable exposure to English, these children would show more rapid growth in English, which might be less established than Armenian. The last prediction was based on the bilingual literature reviewed previously, where environmental characteristics, including parental education and language exposure, as well as child characteristics, including nonverbal IQ and PSTM, have been shown to correlate with vocabulary performance. The novel contribution here was the attempt to assess simultaneously the relative contributions of these multiple characteristics.

\subsubsection{Organization of the Present Dissertation}

The research project presented in this dissertation was comprised of two studies, Study 1 and Study 2. In Study 1, a bilingual vocabulary test was developed and pilot tested. In Study 2, this vocabulary test and other measures were administered to address the main questions of the research. Chapter 2 covers Study 1. In Chapter 3 the method of Study 2 is described. The results of Study 2, as well as the general results of Study 1 are presented in Chapter 4. Finally, the results of both studies are discussed in Chapter 5 . 


\section{Study 1: Development of a Bilingual Vocabulary Test}

The purpose of Study 1 was to develop a bilingual vocabulary test that could be used in the main study, Study 2, to compare vocabulary performance in receptive and expressive modalities of Armenian and English in young bilingual children. The robustness of the vocabulary test was considered of prime importance in this dissertation, because it was the main measure used in Study 2. The stronger this test, the stronger, more accurate, and more defensible the results of the main study would be. Therefore rigorous measures were taken to develop a parallel ArmenianEnglish bilingual vocabulary test that ideally would consist of four parallel independent lists of vocabulary items. These lists could then be used in measuring receptive and expressive vocabularies in Armenian and English in young children, so that reliable comparisons could be made across the two modalities of the two languages. In developing this vocabulary test, careful procedures were employed so that the test would not only be appropriate for use in the main study but also would be commensurate with the fundamental principles appropriate for development of vocabulary assessment measures in young children. The following paragraphs focus on three of the main principles of test development, as articulated in accepted guidelines (American Educational Research Association; American Psychological Association; National Council on Measurement in Education, 1999) and how the vocabulary test developed in Study 1 was compatible with these principles.

A first key principle is to define clearly the specific purpose of the test in order to guide selection of appropriate content and procedures. In this case, the purpose was to measure both receptive and expressive vocabularies in Armenian and English in young children. Consistent with this specific purpose, picture identification and picture naming tasks were chosen for two main reasons as the most appropriate tasks for measuring receptive and expressive vocabularies in young bilingual children. First, these tasks are used often in measuring receptive (e.g., Peabody Picture Vocabulary Test [PPVT; Dunn \& Dunn, 1981; 1997]) or expressive (e.g., Expressive Vocabulary Test [EVT; Williams, 1997]) vocabulary in bilinguals as well as monolinguals. Second, given the age of the participants in the main study and the variable levels of proficiency that could be observed in the two languages of these children, the picture identification and picture naming tasks were considered to be appropriate because they would only require pointing

to the target picture or naming single pictures, which were considered to be within the abilities of 
the least proficient bilinguals. In addition, in developing the initial pool of vocabulary items, measures were taken to identify as many potential items as possible so that the best items suitable for the purpose of the test could be selected.

The second key principle is that tests, including vocabulary tests, are usually structured from easy to difficult, so that during test administration, easier items are introduced first followed by more difficult ones. This principle was followed in developing all four vocabulary lists. As detailed below, careful and extensive measures were taken to initially obtain age of acquisition (AoA) values for the vocabulary items and then to order the vocabulary items based on AoA from easy to difficult in all the four vocabulary lists.

Finally, a third important principle for developing a test in general and a vocabulary test in particular is to specify and use consistent, replicable procedures in administering and scoring the test. Consistent procedures in test administration and scoring ensure that all the participants experience unbiased and equivalent conditions of test taking. This principle was followed vigilantly in the present study. First, careful and detailed procedures of test administration were laid out (see Section 3.3) and then these procedures were followed during test administration and scoring.

Therefore, the parallel bilingual vocabulary test developed in Study 1 and described here was comprised of picture identification and picture naming tasks, the former for testing receptive vocabulary and the latter for testing expressive vocabulary. The development of the test involved seven stages, which were: (1) identifying English picturable vocabulary items and their pictures that potentially could be used in the vocabulary test; (2) obtaining percentages of correct use of the expected labels for the pictures and AoA ratings for their expected labels from adult speakers of Armenian and English who were familiar with children and their vocabulary development; (3) selecting target items with expected and consistent labels in both dialects of Armenian (Western and Eastern) and English; (4) developing four equivalent vocabulary lists of target items arranged in ascending order of their AoA values; (5) selecting foil pictures to accompany the target pictures in the picture identification tasks; (6) programming the picture identification and picture naming tasks in the SuperLab Pro program (Cedrus, Version 2.0.4); and (7) pilot testing the tasks and modifying the vocabulary lists based on the results. Each of these steps is described in turn. 


\subsection{Identifying English Picturable Vocabulary Items}

\subsubsection{Method}

At this stage, picturable English vocabulary items were identified that could be potentially used in the vocabulary test. Three criteria were used. First, the items were appropriate for 4- and 5year-old children. Second, they could be used in both picture identification and picture naming tasks. Finally, they would be equally applicable to Armenian and English. Although these criteria limited the number of potential items, efforts were made to develop as large a pool of potential vocabulary items as possible. Procedures used to meet the three selection criteria are discussed separately.

\subsubsection{Vocabulary Items Appropriate for Young Children}

To identify potential vocabulary items appropriate for 4- and 5-year-old children, the target population of Study 2, two requirements were fulfilled. First, a measure of vocabulary acquisition was used to help decide whether a given vocabulary item was appropriate for these age groups. Second, care was taken to avoid ceiling effects in the vocabulary tasks.

There were two options available to address the first requirement. The first option was to use the frequency of occurrence of vocabulary items in the language and the second option was to use AoA, an estimate of the age at which the vocabulary items were acquired. AoA was chosen as the measure to decide on the appropriateness of the vocabulary items for the target population of the study. This choice was made for several reasons. First, moderate to high correlations, ranging from $r=-.58$ to $r=-.73$, have been reported between AoA and frequency (Carroll \& White, 1973; Morrison, Ellis, \& Quinlan, 1992). Second, AoA provides more straightforward information on the age at which children acquire vocabulary items. Therefore, based on this information, selected items could be classified into different age groups. Third, picture naming studies were available in English, which provided objective or subjective AoA values and a pool of English vocabulary items and their associated pictures (Cycowicz, Friedman, Rothstein, \& Snodgrass, 1997; Snodgrass \& Vanderwart, 1980; Szekely, Jacobsen, D'Amico, Devescovi, Andonova, Herron, et al., 2004). The English vocabulary items, their AoA values, and pictures could then be used in developing the current vocabulary test. Fourth, the items used in English frequency studies were usually derived from written texts. This meant that they were not 
necessarily picturable. Finally, to my knowledge there were no frequency studies or AoA values available for Armenian vocabulary. However, in the present study it was feasible to obtain subjective AoA values for the Armenian vocabulary items used in developing the test, as described in Section 2.2.

The second requirement was to avoid ceiling effects in the vocabulary tasks. Accordingly, vocabulary items were selected from a wide range of AoA values, specifically, from two to 12 years. The inclusion of vocabulary items from higher ages (over five years) was intended to ensure that there were sufficient items in the tasks that would be beyond the vocabulary knowledge of high performing 4- and 5-year-old children.

\subsubsection{Vocabulary Items Appropriate for Picture Identification and Picture Naming Tasks}

The vocabulary items identified at this stage were to be used in both picture identification and picture naming tasks. Therefore, they had to be picturable. In addition, for cost-effectiveness considerations, it was necessary to find available pictures, preferably black-and-white line drawings, for these items.

To find vocabulary items with the features mentioned above, two main sources were identified: (1) standardized English vocabulary tests and (2) picture naming and AoA studies of English vocabulary. These sources not only provided lists of picturable vocabulary items, but some of them provided pictures as well as AoA values for the items. The standardized vocabulary measures used in this study were: Expressive One-Word Picture Vocabulary Test-Third Edition (EOWPVT-III; Brownell, 2000); Peabody Picture Vocabulary Test-Revised (PPVT-R; Dunn \& Dunn, 1981); PPVT-III (Dunn \& Dunn, 1997); EOWPVT-R (Gardner, 1990); and Expressive Vocabulary Test (EVT; Williams, 1997). The AoA studies were Morrison et al. (1997); Stadthagen-Gonzalez and Davis (2006); and Szekely et al. (2004). Pictures were used from three sources: Bonin, Peereman, Malardier, Meot, and Chalard (2003); Cycowicz et al. (1997); and Szekely et al. (2004). The pictures in Bonin et al. were available to the public online. Permission was obtained in writing through electronic mail from the first authors of the other two studies for use of the pictures from their studies (see Appendix A). 


\subsubsection{Vocabulary Items Appropriate for Armenian and English}

Finally, steps were taken to ensure that the identified English vocabulary items were also appropriate for the Armenian tasks. Therefore, translation equivalents in Armenian were obtained for all items. All the English vocabulary items identified at the first stage were translated into Armenian by the researcher, a first-language speaker of Armenian. For these translations the researcher made extensive use of Western and Eastern Armenian dictionaries (e.g., Asmangulian \& Hovhannisian, 1984; Awde \& Maghdashyan, 2003; Samuelian, 1993). The translations were then verified by four fluent adult speakers of Armenian. All the individuals, who were Armenian-English bilinguals, were also parents of young children at the time of the study. Two of them were fluent speakers of Western Armenian and the other two were fluent speakers of Eastern Armenian as their first language. None of these four individuals participated in any other stage of the study. These individuals were asked to identify any items for which they disagreed with the translation equivalents provided by the researcher. There was complete agreement between the researcher and the four Armenian speakers on all Armenian translation equivalents. The English items with translation equivalents in Western and Eastern Armenian, i.e., items with entries in Western and Eastern Armenian dictionaries, were then identified as potential vocabulary items appropriate for English as well as the two Armenian dialects.

\subsubsection{Results}

A pool of 343 potential vocabulary items was developed (see Appendix B for an alphabetical list of these items in English and their translation equivalents in Armenian). The items identified met all the criteria and requirements mentioned above. These potential items were acquired within the range of two to 12 years, based on the English AoA values or norms from standardized tests (see Appendix B, Column 6, AoA). All the items were picturable and their pictures were available as black-and-white line drawings. Finally, all the English items had verified translation equivalents in Armenian (see Appendix B, Column 3, Armenian).

\subsection{Obtaining Common Labels and Age of Acquisition Ratings}

\subsubsection{Method}

The main purpose of this stage was to obtain more information about the 343 possible vocabulary items in addition to the translation equivalents obtained in the previous stage. By 
translating the English items into Armenian in the previous stage, it was ensured that for each English item there was an equivalent in Armenian. However, these translation equivalents did not provide the additional information needed to select potential vocabulary items for both Armenian and English tasks. Therefore the additional information obtained in this stage could be used in selecting target items in the next stage. This information, included: (a) the most common and expected labels in Western and Eastern Armenian and English used to refer to the pictures; (b) the percentages of correct use of these labels in all three languages; and (c) the subjective AoA ratings for these items in all three languages. Although information was available on AoA of the items in English, no studies were found to provide AoA ratings for their Armenian equivalents. In addition, information on AoA values of the English vocabulary items came from different sources, which were not necessarily comparable. Therefore, in this stage, information on common labels and AoA ratings was obtained not only for Armenian but also for English items to ensure the comparability of the information across the two languages. This information was collected using the Labelling and Age of Acquisition Rating task described below.

The Labelling and Age of Acquisition Rating task was designed to address the main purpose of this stage by obtaining the information mentioned above. The participants for this task were 15 preschool and elementary teachers and parents who were speakers of Western Armenian, Eastern Armenian, or English. The reason for having teacher or parent participants was their familiarity with children and their vocabulary development, in general, and their familiarity with ArmenianEnglish bilinguals and their vocabulary development, in particular. Thus, the AoA ratings obtained from these adult raters may reflect implicit knowledge of the unique circumstances and challenges of bilingual vocabulary development in these children. The sample included five fluent speakers of Western Armenian (4 teachers; 1 parent), five fluent speakers of Eastern Armenian (4 teachers; 1 parent), and five first-language speakers of English (all teachers). The participants were recruited from the two Armenian schools in the GTA. An information letter including a consent form (see Appendix C) was distributed among the preschool and elementary teachers after receiving the principals' permission. A similar letter (see Appendix C) was distributed among parents of elementary students, because the number of teachers speaking the Armenian dialects was not sufficient. The teachers and parents willing to participate in this study returned a signed copy of the consent form. A meeting was arranged with each participant at the school. The task was administered individually. 
The materials for this task were black-and-white line drawings each representing one of the 343 possible vocabulary items. The pictures were randomly assigned to five different orders of presentation. The five orders were then randomly assigned to the five participants within each language group. The investigator used a laptop computer, a microphone, and a response form for administering the task and recording the responses. Pictures were presented one at a time in the centre of a 14" Hewlett Packard Notebook display using the SuperLab Pro program, which is software for running experiments that involve presenting visual stimuli on a computer screen. The program was also used for electronic recording of the AoA rating responses. A microphone was attached to the laptop for audio recording the participant's labelling responses. The Audacity program, a free digital audio editor (Sourceforge.net, Version 1.2.6) was used for this purpose. Furthermore, during the task a hard copy of a response form, containing a list of the target items, was used to keep a back-up record of the labelling responses.

Participants in each language group were asked to name each picture in that language with the first label that came to their mind upon seeing the picture. The participants were also asked to rate each label, immediately after labelling the picture, on a 12-point scale based on the age that they thought children speaking their language learned that label. Learning was defined as understanding or using the label in their language. The scale was: (1) less than 2 years, (2) 2 years, (3) 3 years, (4) 4 years, (5) 5 years, (6) 6 years, (7) 7 years, (8) 8 years, (9) 9 years, (10) 10 years, (11) 11 years, and (12) 12 years and older. To indicate their ratings, each participant hit one of the 12 keys on the laptop keyboard labelled accordingly. By hitting each key the responses were automatically recorded on the SuperLab database. Before presenting the task pictures, two other pictures were presented to familiarize the participant with the task.

\subsubsection{Results}

The Labelling and Age of Acquisition Rating task provided two main types of data: (1) mean percentage of use of expected labels for each picture, i.e., correct labels for each picture commonly used by the speakers of each language and (2) mean subjective AoA ratings for these labels. After obtaining the percentage of use of expected labels in each language group separately, the combined mean percentage of use of expected labels for each picture was pooled across all three language groups (see Appendix B, Column 7, M\%). This summary statistic 
indicated the percentage of the participants that identified the pictures correctly and named them with the expected labels.

The task also provided subjective mean AoA values for the expected labels used to name the pictures. To obtain this value, first the mean AoA value in years was obtained for the expected labels for each picture, in each language group separately. Then the mean AoA in years was obtained for all three language groups (see Appendix B, Column 8, M(SD)AoA). The latter was used as a summary statistic and a selection criterion for the target vocabulary items in the next stage. Calculation of Cronbach's alpha showed that inter-rater reliability for the AoA ratings was .80 in the Western Armenian group; .77 in the Eastern Armenian group; .84 in the English group; and .92 in the three language groups combined. These high inter-rater reliabilities among the three language groups justified the combining of the AoA ratings from the three groups to obtain the mean AoA as the primary summary statistic.

A correlation analysis was conducted to investigate the association between the AoA ratings of the items by the three language groups. There were significant correlations of mean AoA ratings for items between Western Armenian and Eastern Armenian, $r(311)=.66, p<.01$; Western Armenian and English, $r(323)=.68, p<.01$; and Eastern Armenian and English, $r(316)=.60$, $p<.01$. In these correlations the degrees of freedom are different and less than 343, i.e., the total number of vocabulary items in the task, because the groups did not identify and label some of the pictures. Correlations were also computed between the subjective AoA ratings obtained from the Labelling and Age of Acquisition Rating task and the English AoA values and vocabulary norms from published studies and tests (for a list of the studies and tests see Appendix B, Footnote 5). The AoA ratings from this study had relatively high correlations with the published AoAs and norms, $r(343)=.70, p<.01$, and with the objective AoA values from Morrison et al. (1997), $r$ $(258)=.71, p<.01$. The correlations among the AoA ratings of the three language groups showed that there was a reasonable consensus among the three groups in rating the vocabulary items for their AoA. In addition, the correlation between the subjective AoA ratings in this study and the ones from published studies is indicative of the reliability of the procedures used in obtaining the AoA ratings in this study. 


\subsection{Selecting Target Items}

\subsubsection{Method}

The main purpose of this stage was to select the target items from the initial pool of 343 items based on the labelling and AoA information obtained in the second stage. However, before selecting target items, any items with different labels in the two Armenian dialects $(n=16$, see Appendix B, Column 2, items marked with an asterisk*) were excluded. These items were excluded because the final vocabulary test was intended for use in a study where speakers of both Armenian dialects participated and where the number of children speaking each dialect could not be controlled. Therefore, by eliminating these items the potential confound introduced by dialect differences could be minimized.

The target items were then selected from the remaining 327 items using one criterion, i.e., the percentage of use of expected labels in each language group. Ideally items labelled consistently with expected labels would be chosen. However, this meant that the target items would end up being from lower AoA values, risking ceiling effects, because usually the items labelled consistently were simpler ones with lower AoA values. Therefore, to ensure that items with higher AoA values were also represented in the final tasks, a minimum selection criterion of $40 \%$ use of expected labels in each language group was used. This ensured that at least two out of five raters in each language group identified the picture correctly and named it with an expected label, so that eventually the items would be potentially recognizable by at least some children when used to measure their vocabulary performance.

\subsubsection{Results}

Overall, 256 items were selected from the initial pool of 343 items as possible target items for the vocabulary lists. These items were used in developing the four parallel and independent vocabulary lists, as discussed below.

\subsection{Developing Four Parallel and Independent Vocabulary Lists}

\subsubsection{Method}

The main purpose of this stage was to develop four parallel and independent 50-item vocabulary lists (A, B, C, and D), equated on mean AoA values, which could be presented in picture 
identification and picture naming tasks in Armenian and English. To develop the four lists, each with 50 different items, 200 target items would be selected, as described below, from the 256 items identified in the previous stage. Therefore, by having 200 target items, four 50 -item independent lists could be developed with no repetitious items. Also, there would be no overlap in the items used in picture identification and picture naming tasks in both languages. The four lists (A, B, C, and D), could then be counterbalanced across the four vocabulary tasks, i.e., Armenian picture identification, Armenian picture naming, English picture identification, and English picture naming. Each child would, therefore, be tested on all four lists, one list in each of the four tasks. Children would be assigned randomly to one of four different list-task combinations, developed by the use of a balanced $4 \times 4$ Latin Square. To give an example, one of the four list-task combinations developed thus would be 'A-B-D-C'. This list-task combination would be randomly assigned to one of the participants in a block of four. Therefore, for this participant, List A would be used for assessing Armenian picture identification, List B for assessing Armenian picture naming, List D for assessing English picture identification, and List C for assessing English picture naming. A fixed order of task administration would be used, as described in Section 3.3.1.

Of the 256 items identified in the previous stage, 200 items were selected as target items and divided into four parallel lists (A, B, C, and D) using stratified random assignment. To develop these four lists the following steps were taken. First, because of the high number of items with AoA values of two and three years, only those with 100\% expected labels in all three languages $(n=90)$ were assigned to the lists and the rest $(n=16)$ were eliminated. The use of these items ensured that about $45 \%$ of the total items would be easy enough for the participants; therefore minimizing the risk of floor effects especially in the picture naming tasks. A potential disadvantage of this approach was that these items might be easy for the participants, resulting in less individual variability. Second, one item (banana) was randomly excluded from the 90 items selected in the first step, so that with these 89 items and the remaining 111 items selected from higher AoA values, the total number of target items would add up to 200. Third, the 200 selected target items were arranged into groups of four in an ascending order based on their overall mean AoA values. In other words, the first four items with the lowest mean AoA values made up the first group of items, and then the next lowest four items followed them until all the selected items 
were assigned to groups of four. Finally, each item in each group was randomly assigned to one of the four lists (A, B, C, and D). These lists are presented in Appendix D, Table 1.

\subsubsection{Results}

Each of the four lists contained 50 target items arranged in ascending order of AoA (see Appendix D, Table 1, items 1 to 50). The procedures described above were taken to ensure that the lists were equivalent in rated AoA. However, to provide statistical evidence for equivalence of the lists on rated AoA, a one-way analysis of variance (ANOVA) was conducted to compare the mean AoA values of the items in the four lists. The four vocabulary lists did not differ significantly in their mean AoA values. Table 1 presents the means and standard deviations for the AoA values in years in the four lists. A similar analysis was conducted to compare the four lists on their overall percentages of use of expected labels. There were no significant differences among the lists. Table 1 also presents the means and standard deviations for the percentages of use of expected labels in the four lists. 
Table 1.

Means (M) and Standard Deviations (SD) for Age of Acquisition (AoA) and Labelling in the Four Lists

\begin{tabular}{|c|c|c|c|c|}
\hline & \multicolumn{4}{|c|}{ Lists } \\
\hline & List A & List B & List C & List D \\
\hline & $M(S D)$ & $M(S D)$ & $M(S D)$ & $M(S D)$ \\
\hline AoA & $4.05(1.13)$ & $4.07(1.16)$ & $4.06(1.12)$ & 4.07 (1.14) \\
\hline Labelling & $92.93(10.55)$ & $92.79(9.69)$ & $93.46(10.73)$ & $91.60(11.64)$ \\
\hline
\end{tabular}

Note. AoA $=$ Age of Acquisition values in years for the target items in each of the four lists (A, $\mathrm{B}, \mathrm{C}$, and D); Labelling = Overall percentage of use of expected labels for the target items in each of the four lists. 


\subsection{Selecting Foil Pictures for the Identification Tasks}

\subsubsection{Method}

The main purpose of this stage was to find appropriate foil pictures for the 200 target items, so that the target items could be used not only in picture naming but also in picture identification tasks. The pictures representing the target items could be used singly in the picture naming tasks. However, to use these pictures in the picture identification tasks they would need to be accompanied by at least three non-target pictures, which would act as foils for the target picture.

One criterion was used to select three foil pictures for each picture representing a target item. The criterion was that the mean AoA ratings of the foil items were within \pm 1 year of the AoA of the target item.

As discussed earlier, only 200 items were selected as targets from the original pool of 343 items. The remaining 143 items were then used as foils. An advantage of using the pictures of these items as foils was that the AoA ratings for these items were obtained under the same conditions and from the same participants as those for the target items.

The total number of foil pictures needed for all four lists was 150 . The number of available foils was 143; therefore seven more items were needed to increase this number to 150 . Thus, different illustrations of seven foil items (e.g., two visually distinct pictures of panda) were chosen to increase the number of foils to 150 .

There were three main advantages to having the 150 foils for the two identification tasks. First, all these items had AoA values comparable to those of the target items. Second, there were available pictures for these items. Third, most of these pictures were recognizable, as evidenced by the fact that they had already been labelled by adult speakers of Armenian and English. However, there was one main disadvantage to this choice. Having only a total of 150 foils for four 50-item lists and counterbalancing these lists meant that the same foil pictures would appear in both Armenian and English picture identification tasks. Although the foil pictures were not to be named in these tasks, repeated exposure might increase visual familiarity. However, as will be described later, procedures were employed so that the same set of three foil pictures did not appear together. This was intended to decrease the familiarity of repeated items. 
To assign three foils to each target item in the four vocabulary lists (A, B, C, and D) the following procedure was used. First, the total 150 foils were stratified by their AoA values starting from the lowest and moving towards the highest. Then the first three foils with the lowest AoAs were assigned to the first target item with the lowest AoA in the four lists. This ensured that the targets and their assigned foils were matched as closely as possible in AoA. The same procedure was followed until each of the 50 target items in the lists was assigned three foils. This procedure resulted in assigning the same three foils to the corresponding target items in the four lists. For example, 'hand, bread, and spoon' were the first three foils with the lowest AoAs, which were assigned to the first target item in the four lists, i.e., 'cat' in List A, 'apple' in List B, 'dog' in List C, and 'ball' in List D. Therefore, to prevent the same three foil pictures always appearing together, the 12 foils for each group of four consecutive target items were randomly assigned to different orders. For example, as shown in Table 2 in the four panels of List A, List B, List C, and List D, the 12 foils, i.e., 'hand, spoon, bread, glass, hair, house, banana, finger, sleeping, mitten, car, and boot' appeared with different targets and in different combinations in the four lists. During these procedures, care was taken to ensure that the foils remained within \pm 1 year of AoA of their assigned targets. Finally, as the 50 items in each list were divided into groups of four, the last two items (items number 49 and 50) were left out. Therefore the same foils were assigned to the last two items in each list, because if the order was changed the AoAs of the foil items would no longer fall within a range of \pm 1 year of the AoAs of their target items.

In the final step, the four items in each group, consisting of a target item and its three foils, were randomly assigned to one of the four positions or corners. These four corners represented the position of the target item and its three foils on the picture identification task trial page. For the target items and their foils see Appendix D, Tables 2 to 5, items 1 to 50 in each list. 
Table 2.

First Four Consecutive Target Items in Each of the Four Lists with Their Respective Foils

\begin{tabular}{|c|c|c|c|c|}
\hline & Targets & & Foils & \\
\hline \multicolumn{5}{|l|}{ List A } \\
\hline & 1. cat & bread & hand & spoon \\
\hline & 2. nose & glass & hair & house \\
\hline & 3. table & banana & finger & sleeping \\
\hline & 4. ear & mitten & boot & car \\
\hline \multicolumn{5}{|l|}{ List B } \\
\hline & 1. apple & car & sleeping & glass \\
\hline & 2. shoe & bread & mitten & finger \\
\hline & 3. book & boot & hair & hand \\
\hline & 4. rabbit & banana & house & spoon \\
\hline \multicolumn{5}{|l|}{ List C } \\
\hline & 1. $\operatorname{dog}$ & mitten & sleeping & hair \\
\hline & 2. sun & banana & car & bread \\
\hline & 3. cheese & boot & spoon & glass \\
\hline & 4. fish & house & hand & finger \\
\hline \multicolumn{5}{|l|}{ List D } \\
\hline & 1. ball & house & finger & mitten \\
\hline & 2. door & hand & sleeping & car \\
\hline & 3. chair & banana & bread & hair \\
\hline & 4. horse & glass & spoon & boot \\
\hline
\end{tabular}




\subsubsection{Results}

As described earlier, the mean AoA values of the foils were within \pm 1 -year of the AoA of the targets. However, to provide statistical evidence for this, the mean AoA of the target items and their foils in each list were compared using paired-samples $t$-tests. As shown in Table 3, none of the four tests were significant. Overall, these results suggested that the targets and their foils in all four lists did not differ significantly in their mean AoA values.

\subsection{Programming the Vocabulary Tasks in SuperLab}

After developing the vocabulary lists, the pictures representing the target items in each of the four lists were downloaded in the SuperLab Pro program separately as stimuli for the picture naming task (see Figure 1 for an example). In addition, the target pictures and their foils in each of the four lists were downloaded together as stimuli for the picture identification task (see Figure 2 for an example). However, before downloading the pictures for the picture identification task, each target picture and its three foils were arranged in the four corners of the page, using the DRAW option from OpenOffice.org program (Version 2.4.1), which provides a tool for managing graphics. Use of the SuperLab program made it possible to present the trials in each of the tasks one at a time on a laptop computer display and to record the responses. The order of presenting the target trials in each list followed the order determined in the development of the lists (see Appendix D, Table 1). 
Table 3.

Means (M) and Standard Deviations (SD) for Age of Acquisition (AoA) Values in Years for the Targets and their Foils in the Four Lists and Results of Tests of Significance

\begin{tabular}{|c|c|c|c|c|c|}
\hline & \multicolumn{2}{|c|}{ Items } & & & \\
\hline & Targets & Foils & \multicolumn{3}{|c|}{ Test of Significance } \\
\hline & $M(S D)$ & $M(S D)$ & $d f$ & $t$ & $p$ \\
\hline List A & $4.05(1.13)$ & $4.08(1.20)$ & 49 & -.740 & .463 \\
\hline List B & $4.07(1.16)$ & $4.08(1.20)$ & 49 & -.380 & .706 \\
\hline List C & $4.06(1.12)$ & $4.08(1.20)$ & 49 & -.518 & .607 \\
\hline List D & $4.07(1.14)$ & $4.08(1.20)$ & 49 & -.328 & .744 \\
\hline
\end{tabular}

Note. $d f=$ degrees of freedom, $t=t$-statistic in $t$-test; $p=p$-value. 


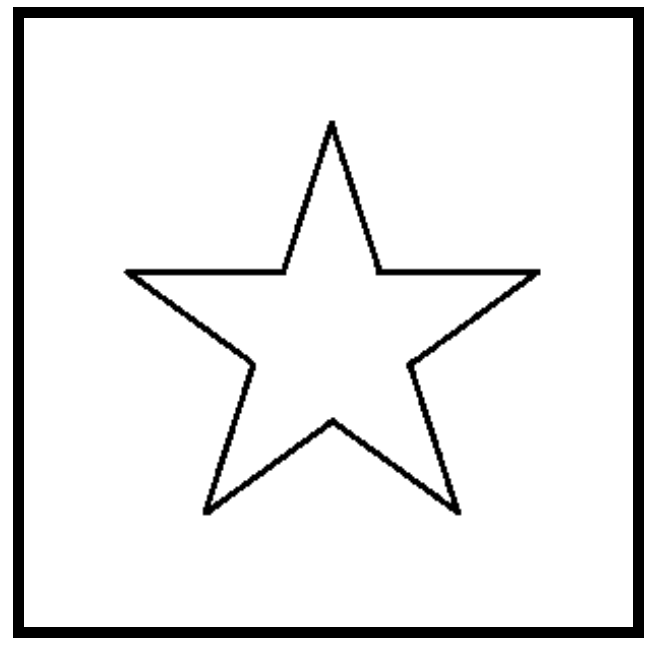

Figure 1. A sample of the picture naming task trial with 'star' as the target item. (From the World Wide Web at http://crl.ucsd.edu/ aszekely/ipnp/. Reprinted with permission.) 


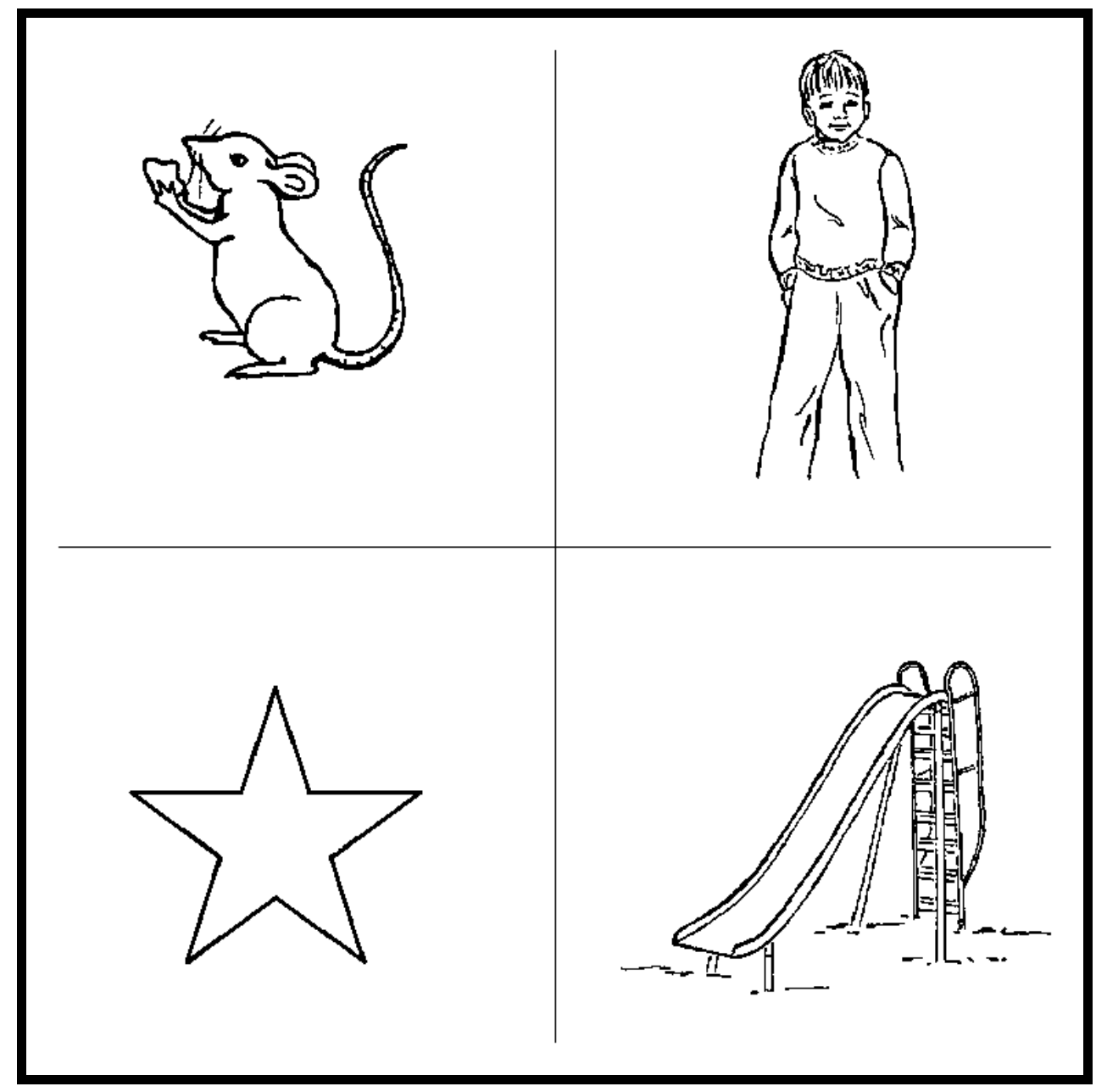

Figure 2. A sample of the picture identification task trial with 'star' as the target item. (From the World Wide Web at http://crl.ucsd.edu/ aszekely/ipnp/. Reprinted with permission.) 


\subsection{Pilot Testing the Vocabulary Tasks}

\subsubsection{Method}

After downloading the picture identification and picture naming tasks in SuperLab, the bilingual vocabulary test was ready for pilot testing to examine the time frame for administration of the tasks, the clarity of the instructions, and the suitability of the pictures. The vocabulary tasks were pilot tested on six Armenian-English bilingual children. The participants were recruited from the Armenian Saturday School in the GTA. After meeting with the Saturday School principal, permission was obtained to distribute the information letter containing the consent form (see Appendix E) among parents of children attending the school. Two selection criteria were included in the information letter, i.e., being four or five years of age and having Armenian spoken at home by at least one parent. The parents who were willing to allow their child to participate in the study were asked to sign a copy of the information letter. Six families returned their signed consent forms. The six 4- and 5-year-old children who participated in the pilot study provided their assent (see Appendix E for child assent script). Two of the children were four years old and the others were five years old. In each age group, one child spoke Eastern Armenian and the rest spoke Western Armenian.

The results of the pilot study raised the possibility of encountering ceiling effects, especially in the picture identification tasks. The participants scored relatively high in Armenian picture identification $(M=64.66 \%, S D=12.24 \%)$ and English picture identification $(M=75.00 \%, S D=$ $15.63 \%$ ) tasks. Therefore to be on the safe side and to avoid potential problems in the main study, more items with AoA values of six years or older were added to the lists.

The vocabulary items for higher age groups were selected from AoA studies on English vocabulary (Carroll \& White, 1973; Morrison et al., 1997) and standardized English vocabulary tests, specifically, the PPVT-III (Dunn \& Dunn, 1997), the EOWPVT-R (Gardner, 1990), and the EVT (Williams, 1997). The Armenian translation equivalents for these additional items were obtained using the same procedure outlined in Section 2.1.1.3; however AoA ratings could not be obtained through the same procedure outlined in Section 2.2.1 because it was impractical to expect raters to return for a second rating session. Instead, the English AoA values for these additional items were obtained from the published sources used to select these items. As discussed in Section 2.2.2, the high correlations between the AoA ratings from this study and 
those from published sources were thought to justify the use of these values in the absence of adult ratings for this small number of additional items. Therefore, the addition of five unrated items to the end of each list, as described in the following paragraph, was thought to be reasonable, given the assumption that testing for most children would be discontinued before the last items in the lists (see Section 3.3.2.2).

The sources mentioned above provided only 35 new items with the required features. Twenty of these items were selected randomly as target items and the remaining 15 items were considered as foils for these targets. The target items were added to the end of the four lists. In doing so, first the target items were arranged in ascending order of their AoA values or norms. Then, starting from the top of the list, the 20 target items were divided into five groups of four items. The four items in the first group of four were randomly assigned to each of the four lists. The same procedure was used for the next four groups of four. As a result, five target items with ascending AoA values or norms were added to the end of each list. In the next step, to assign the foils to the target items, first the foils were arranged in ascending order of their AoA values or norms. Then starting from the top of the list the 15 foils were divided into five groups of three. The first group of three was assigned to the first target item added to the original list. The same procedure was followed for the next four groups of target items. As shown in Appendix D Tables 2 to 5, the same foils were assigned to the same target items in the lists, to make the foils as comparable as possible in their AoA values with the target. For example, 'fingerprint, banjo, igloo' were assigned to target item number 51 in all four lists. However, different orders were used in the four lists in presenting the foils in the four corners of the picture identification task trial page. As a final step, the tasks in SuperLab Pro were modified accordingly.

\subsubsection{Results}

Four vocabulary lists were developed each with 55 items. After adding five items to each of the four original lists, a one-way ANOVA was conducted to find out whether the lists remained equivalent. The four vocabulary lists did not differ significantly in their mean AoA values with the addition of new items. The means and standard deviations for the mean AoA values in years in the four new lists were: List A, $M=4.39, S D=1.55$; List B, $M=4.38, S D=1.52$; List C, $M=$ $4.39, S D=1.55$; List $\mathrm{D}, M=4.41, S D=1.60$. The new vocabulary lists were re-programmed in 
SuperLab Pro as picture identification and picture naming tasks. For a complete list of the target items and their foils see tables 2 to 5 in Appendix D.

\subsection{Conclusion}

Following the rigorous and standard procedures outlined in the present chapter, a bilingual Armenian-English vocabulary test was developed, which was appropriate for 4- and 5-year-old Armenian-English bilingual children. These procedures were in line with some of the main standards for test development (see American Educational Research Association; American Psychological Association; National Council on Measurement in Education, 1999).

The vocabulary test consisted of four lists of vocabulary items, each with 55 target items. The four lists were counterbalanced across the four tasks, i.e., Armenian picture identification, Armenian picture naming, English picture identification, and English picture naming. The four lists were equivalent in rated mean AoA values of their constituent items. The items in each list were arranged in ascending order of their AoAs. Therefore each list started with simple items and became progressively more difficult. None of the target vocabulary items were repeated across these four lists and none of them were used as foils. For a list of the target items in the four lists see Appendix D, Table 1. The targets in the table appear in the same order as presented in the tasks. As shown in the table, all the target items are nouns with the exception of few verbs. The inclusion of verbs might raise concern over the equivalence of the target items in terms of their semantic features. However, this concern is minimized by the fact that, first, the test was not used to address the question of semantic differences in the target items; second, the difficulty levels of the verbs were equivalent to the noun items appearing in corresponding positions in other tasks; and third, the PPVT (Dunn \& Dunn, 1981; 1997), as a reliable model for developing a picture identification task also includes some verbs in addition to many nouns. In fact, all the verbs were originally selected from the PPVT; see Section 2.1.1.2 for more details on the development of the original pool of potential vocabulary items. 


\section{Study 2: Method}

This study had two objectives: (1) to investigate differences in Armenian and English vocabulary growth in Junior Kindergarten (JK) and Senior Kindergarten (SK) children who were exposed to Armenian and English at school and (2) to investigate individual differences in participants by examining predictors of vocabulary performance. Two groups of Armenian-English bilingual kindergarteners participated in this study. Both groups were tested twice (Time 1 and Time 2) over a six-month interval on Armenian and English picture identification and picture naming tasks. The first testing (Time 1) was done in October - November 2008, two months after the start of the school year. The second testing (Time 2) was done in April - May 2009, towards the end of the school year. In addition, at Time 1, data were obtained from both groups on relevant environmental and child characteristics using a parental questionnaire and direct assessments.

The present chapter starts with a power analysis. Next the focus is on a description of the participants, their characteristics, and their recruitment. Following this, general procedures and the timeline of the study are described. Finally, the vocabulary task measures, additional measures, and program fidelity are presented.

\subsection{Sample Size and Power Analysis}

Ideally, effect sizes for growth in Armenian and English picture identification and picture naming would be needed to estimate the required sample size and perform a power analysis. However, in the absence of this information, effect sizes were calculated from a previously described study on vocabulary growth in Spanish-English bilingual children by Winsler et al. (1999). The effect sizes determined from this study represented vocabulary growth from Time 1 to Time 2 over a six-month interval. It was assumed that the vocabulary effect sizes from this study would be comparable to those of the present study because of a similar test interval and participant age. In addition, Spanish, like Armenian, was a minority language.

Portney and Watkins (2000, p. 708) suggest a paired $t$-test formula for the calculation of $d$ based on the paired scores in a repeated measures design. This formula was used in calculating the effect sizes. The data reported in Winsler et al. (1999) on Spanish and English picture identification and picture naming tasks were used as the paired scores. Then, a correlation of .50 was assumed between Time 1 and Time 2 scores, because the correlation, which was necessary 
for the effect size calculation, was not reported in Winsler et al. A correlation of .50 was a conservative choice given that it was a modest estimate of the correlation between Time 1 and Time 2, which did not run the risk of decreasing the sample size and the power of the study that might occur if a higher, less conservative correlation was chosen. Using this estimate, the $d$ values were calculated for growth in the four vocabulary tasks. The effect sizes obtained from the age group comparable to the JK group in the present study were: $d=1.6$ for Spanish picture identification, $d=.4$ for Spanish picture naming, $d=1.5$ for English picture identification, and $d$ $=1.1$ for English picture naming. The effect sizes from the age group comparable to the SK were: $d=.8$ for Spanish picture identification, $d=.1$ for Spanish picture naming, $d=.6$ for English picture identification, and $d=.5$ for English picture naming. The mean of these effect sizes, i.e., .8 was considered as a reasonable estimate of effect size for the present study. With $p$ $=.05$ and $n=20$ in each group, power was approximately .70 for within-group vocabulary growth and greater than .90 for full-sample $(N=40)$ vocabulary growth.

\subsection{Participants}

Forty-two bilingual kindergarteners who were attending Armenian-English bilingual schools participated in this study. They were from families where Armenian was spoken at home by at least one parent. Twenty children (40.0\% male) with a mean age of 51.6 months were attending JK and 22 children (54.5\% male) with a mean age of 63.1 months were attending SK. At Time 2, the mean age of JK children was 57.6 months and the mean age of SK children was 69.2 months.

The participants were attending one of the two private Armenian-English bilingual schools in the GTA. The majority of participants $(78.6 \%)$ came from the school with the larger enrolment, with about 50 children in the JK and SK grades. The rest were from the school with the smaller enrolment, with about 20 children in the JK and SK grades. These schools provided a balanced Armenian-English bilingual program for kindergarteners, where each language was taught for half of the school day by a separate teacher. This program offered a unique opportunity to investigate bilingual vocabulary growth in children because this kind of program, compared with a monolingual majority-language program, was beneficial for the development of both languages of bilingual children (Barnett et al., 2007). 


\subsubsection{Participant Characteristics}

To investigate individual differences, information was collected at Time 1 from all participants on environmental and child characteristics related to vocabulary growth. Before providing a detailed description on the measures and procedures employed to obtain this information, a summary of relevant demographic information is presented in Table 4, which includes but is not limited to the information on environmental and child characteristics used in the analyses of individual differences. The environmental characteristics intended for analyses of individual differences included parental years of education, language exposure, and language value; and the child characteristics included nonverbal cognitive ability (nonverbal IQ) and phonological shortterm memory (PSTM). None of these characteristics were used to determine inclusion or exclusion of the participants. In fact, it was important to have variability among the participants with respect to these characteristics so that they could be used in subsequent analyses to explain individual differences.

The information in Table 4 includes parental years of education, language exposure, language value, nonverbal IQ, PSTM, age of participants at Time 1 and Time 2, length of residence of parents in Canada, and age of parents. Except for nonverbal IQ and PSTM, which were assessed directly, the rest of the information was obtained using a researcher-developed parental questionnaire, the 'Home Language Use Questionnaire' (see Appendix F). This questionnaire was intended to be completed by a parent (or caregiver) in approximately 15 minutes. In devising this questionnaire, the investigator consulted similar questionnaires developed by GutiérrezClellen and Kreiter (2003), Kan (unpublished), and Geva (unpublished). Further details on the measures and procedures used to collect the information in Table 4, specifically the information on environmental and child characteristics, are provided in Sections 3.2.2.1 through 3.2.2.2. 
Table 4.

Means (M), Standard Deviations (SD), and Ranges for Participant Characteristics in Junior (JK) and Senior Kindergarten (SK) Grades, and Results of Tests of Significance

\begin{tabular}{|c|c|c|c|c|c|}
\hline \multirow[b]{3}{*}{ Measure } & \multicolumn{2}{|c|}{ Grade } & \multirow{2}{*}{\multicolumn{3}{|c|}{ Test of Significance }} \\
\hline & $\mathrm{JK}(n=20)$ & $\mathrm{SK}(n=22)$ & & & \\
\hline & $\begin{array}{l}M(S D) \\
\text { Range }\end{array}$ & $\begin{array}{c}M(S D) \\
\text { Range }\end{array}$ & $d f$ & $t$ & $p$ \\
\hline Parental Years of Education ${ }^{a}$ & $\begin{array}{l}15.71(2.65) \\
10.0-20.0\end{array}$ & $\begin{array}{l}17.44(2.90) \\
11.0-24.0\end{array}$ & 36 & -1.926 & .062 \\
\hline \multicolumn{6}{|l|}{ Language Exposure } \\
\hline Armenian & $\begin{array}{l}76.85(22.43) \\
35.0-100.0\end{array}$ & $\begin{array}{c}65.80(32.06) \\
1.3-100.0\end{array}$ & 40 & 1.281 & .207 \\
\hline English & $\begin{array}{c}15.69(18.98) \\
0.0-65.0\end{array}$ & $\begin{array}{c}32.28(31.29) \\
0.0-86.7\end{array}$ & $35.08^{\mathrm{b}}$ & -2.098 & .043 \\
\hline Other & $\begin{array}{l}7.45(17.11) \\
0.0-65.0\end{array}$ & $\begin{array}{l}1.79(6.29) \\
0.0-27.5\end{array}$ & $23.63^{\mathrm{c}}$ & 1.396 & .176 \\
\hline \multicolumn{6}{|l|}{ Language Value } \\
\hline Armenian & $\begin{array}{c}11.15(1.04) \\
9-12\end{array}$ & $\begin{array}{c}11.14(1.24) \\
9-12\end{array}$ & 40 & 0.038 & .970 \\
\hline English $^{\mathrm{d}}$ & $\begin{array}{c}11.11(1.56) \\
6-12\end{array}$ & $\begin{array}{c}11.05(1.24) \\
8-12\end{array}$ & 38 & 0.130 & .897 \\
\hline Nonverbal IQ & $\begin{array}{c}119.40(13.68) \\
102-151\end{array}$ & $\begin{array}{c}110.91(13.24) \\
83-147\end{array}$ & 40 & 2.042 & .048 \\
\hline PSTM & $\begin{array}{c}37.59(13.17) \\
7.4-63.0\end{array}$ & $\begin{array}{l}42.42(13.31) \\
18.5-66.7\end{array}$ & 40 & -1.180 & .245 \\
\hline Age (Time 1) & $\begin{array}{l}51.65(2.58) \\
47.0-57.0\end{array}$ & $\begin{array}{l}63.15(3.43) \\
58.5-69.0\end{array}$ & 40 & -12.170 & $<.001$ \\
\hline Age (Time 2) & $\begin{array}{l}57.60(2.61) \\
52.5-63.0\end{array}$ & $\begin{array}{l}69.20(3.29) \\
65.0-75.0\end{array}$ & 40 & -12.562 & $<.001$ \\
\hline Years in Canada ${ }^{e}$ & $\begin{array}{c}14.41(10.56) \\
1.0-33.5\end{array}$ & $\begin{array}{c}20.12(8.28) \\
3.0-33.0\end{array}$ & 35 & -1.844 & .074 \\
\hline Age of Parents ${ }^{\mathrm{f}}$ & $\begin{array}{l}37.31(5.54) \\
31.0-49.0\end{array}$ & $\begin{array}{l}39.45(4.53) \\
31.5-50.5\end{array}$ & 37 & -1.319 & .195 \\
\hline
\end{tabular}


Note. Parental Years of Education = Mean of total years of parents' education; Language Exposure $=$ Parental ratings of mean percentages of use of languages at home (Armenian, English, and other) by parents with the child and by the child with parents and siblings; Language Value $=$ Score ranging from 3 to 12 for each language on questions completed by parents on the importance of Armenian and English language proficiency, literacy and identity; Nonverbal IQ = Nonverbal cognitive ability represented by the standard score $(M=100 ; S D=$ 15) from the Brief IQ Screener, Leiter International Performance Scale-Revised; PSTM = Phonological short-term memory represented by percent correct score from the Hebrew Pseudoword PSTM Task; Age = Age of participants in months at two times of testing; Years in Canada $=$ Mean years of residence of parents in Canada; Age of Parents = Mean years of parents' age; $d f$ $=$ degrees of freedom; $t=t$-statistic in $t$-test; $p=p$-value.

${ }^{a}$ Sample size is 19 for JK and SK, because of missing data.

${ }^{b, c}$ Equal variances not assumed.

${ }^{\mathrm{d}}$ Sample size is 19 for JK and 21 for SK, because of missing data.

${ }^{\mathrm{e}}$ Sample size is 17 for $\mathrm{JK}$ and 20 for SK, because of missing data.

${ }^{\mathrm{f}}$ Sample size is 19 for JK and 20 for SK, because of missing data. 


\subsubsection{Participant Recruitment}

All participants received informed consent from their parents prior to the study. Upon receiving approvals from the Office of Research Ethics of the University of Toronto and the Board of Trustees of each school, the investigator met with the school principals to obtain permission to distribute an information letter containing the consent form (Appendix G, Consent Form Time 1) and a parental questionnaire (Appendix F) to parents of all JK and SK students. The letter included information about the study, the nature of the tasks, the questionnaire used for data collection, the expectations, time commitment, and the voluntary nature of participation. It also included one question addressing the single selection criterion, which was whether Armenian was spoken at home by at least one parent. The question was: Do parents speak Armenian at home? The parents checked their answer on one of the following choices: $\square$ Yes, at least one parent, $\square N o$. If the answer was Yes, the parents willing to participate in the study were asked to sign one of the two copies of the consent form, complete the parental questionnaire, and return them to the school sealed in an envelope provided with the questionnaire and forms. All participants whose parents gave consent met the selection criterion and were included in the study. No other inclusion/exclusion criteria were used.

\subsubsection{Environmental Characteristics}

Parental education, language exposure, and language value have been proposed as environmental characteristics that correlate with vocabulary growth (e.g., Guardado, 2002; Hoff \& Naigles, 2002; Pearson et al., 1997). As mentioned earlier, information on these characteristics was obtained using the 'Home Language Use Questionnaire'.

\subsection{Parental Education}

Parental education was measured as a composite score by obtaining the mean of the total years of education of both parents. However, when there was missing information for one of the parents, the value for the other parent was used instead.

As shown in Table 4, the statistical analyses revealed no significant differences between JK and SK in the total years of parental education. However, the means for both grades indicated that the parents had relatively high levels of education. 


\subsection{Language Exposure}

Three composite scores were used as a measure of language exposure at home in three language groups, i.e., Armenian, English, and other. These scores were obtained from five questions completed by parents on the parental questionnaire. These questions separately addressed the percentages of use of Armenian, English, and other languages at home by parents with the child and by the child with parents and siblings. The five questions, addressing language exposure in Home Language Use Questionnaire (Appendix F), were:

1. What language(s) does the father use with the child? _ $\%$ Armenian _ $\%$ English _ $\%$ Other

2. What language(s) does the mother use with the child? _ $\%$ Armenian _ $\%$ English _ $\%$ Other What language(s) does the child use in each of the following settings?

3. With his/her father

4. With his/her mother

5. With his/her siblings

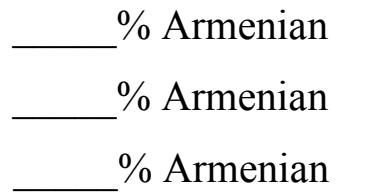

$\%$ Armenian

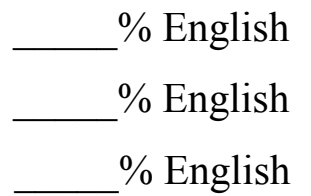

$\%$ English
$\%$ Other $\%$ Other $\%$ Other

The decision to obtain the composite scores from these specific questions was based on the assumption that, for young children, parents are the main source of spoken language input at home, and in the same way, parents and siblings are the most constant individuals at home with whom the child might interact. In fact, the questionnaire also provided two questions regarding use of languages by the child with grandparents and playmates. However, in calculating the composite scores, these questions were not included, because the information provided was not complete, due either to missing data or to the inapplicability of the questions to some families.

To calculate the exposure composite score in each of the three language groups, two separate means were obtained for each language. The first mean, obtained from the first two questions, represented the mean percentage of use of that language by parents with the child. The second mean, obtained from the last three questions represented the mean percentage of use of that language by the child with parents and siblings. The final composite score for exposure was the average of these two means for each language group separately, Armenian, English, and other. If there was missing information for one or more questions, a mean of the values available was used instead.

In addition, correlations were computed for the two means used in calculating the composite exposure score for each language. The two means were mean percentage of use of the language 
by parents with the child and mean percentage of use of the language at home by the child with parents and siblings. The results showed high correlations for each language, ranging from .75 to 93. These high correlations justified the use of the two means in the composite score for each language.

Ideally, to develop a measure of language exposure, observations should be made of the use of the target language by different individuals with the child and vice versa in different environments and conditions. Thus, the approach taken in this study to measure language exposure had its limitations, because it was based on the subjective ratings of parents. However, as mentioned in Chapter 1, parents are in a position to provide reliable ratings of their children's language status including language use and proficiency. This claim has been supported by significant correlations between parental ratings of language use and children's actual use of syntactic structures in their spontaneous narratives (Gutierrez-Clellen \& Kreiter, 2003).

Furthermore, considering feasibility issues, this approach provided a practical way of getting the information needed in a timely manner.

As shown in Table 4, independent samples $t$-tests on Armenian, English, and other exposure revealed a significant difference between JK and SK only in their English language exposure, $t(35.08)=-2.098, p=.043$. This indicated that the SK children had significantly higher exposure to English at home than the JK children. An alternative statistical approach would have been to use analysis of variance (ANOVA) with language exposure as the within-subjects variable with three levels (Armenian, English, other). However, the problem with both analyses was that the scores in percentages were not independent of each other. To address this problem, compositional analysis (Pennington, James, McNally, Pay, \& McConachie, 2009) was a better choice, but because of the different sample sizes in the two comparison groups, this analysis could not be performed on the available data. Therefore, three independent samples $t$-tests were performed to show the differences between JK and SK in exposure in Armenian, English, and other languages separately.

The comparison of the scores in the three languages, as shown in Table 4, revealed some specific features of the participants' language environments. First, there was large variability among the participants in the extent to which they were exposed to and used Armenian and English at home. Second, the overall means suggested that the participants in both grades had higher home 
exposure to Armenian than to English. Third, in both grades some children were exposed to and experienced a third language at home, in addition to Armenian and English. One child was exposed to Armenian and another language with no reported English language exposure at home. Exposure to a third language was present in $20 \%$ of JK families and in $9 \%$ of SK families.

\subsection{Language Value}

The total scores obtained from the answers to questions 11, 13, and 15 and for 12, 14 and 16 (see Appendix F) were used as measures of language value for Armenian and English, respectively. The language value score for each language ranged from three to 12, as each of the three items was rated on a 4-point scale. The total score in each language represented the importance of Armenian or English language proficiency, literacy, and identity from the parents' perspective. For the language value analysis, the scores were reversed because, as seen in the questionnaire, the first and most positive choice, which was "Strongly agree," was scored 1 whereas the last choice, which was the most negative one, was scored 4 . Therefore to have the highest value for the most positive responses and vice versa, the scoring was reversed.

As shown in Table 4, the statistical analyses revealed no significant differences between the groups on language value for either Armenian or English. Although it appears that both groups considered proficiency, literacy, and identity in both languages to be of equal importance, this might be an artifact of the ceiling effects observed in the language value scores. This suggests there may be limited effectiveness in this measure in capturing possible variability among the respondents.

Although the measure for assessing language value was not sensitive to variability among respondents, the descriptive information indicated that parents showed high value for both languages. This descriptive information was obtained from written responses to open-ended questions in the parental questionnaire. In one question, the parents provided their reasons for sending their children to the Armenian-English schools. Almost all the parents believed that sending their children to these schools would help them to develop and keep their Armenian identity, learn about their culture and heritage, learn reading and writing in Armenian, and have Armenian friends. For example one parent wrote: 
"She can identify herself as an Armenian. To understand what it means to be an Armenian. To speak, read and write in Armenian. Learn about our religion and have Armenian friends."

In addition, some parents mentioned the opportunity that the school provided to learn both Armenian and English. One parent wrote:

"In an Armenian school, my child learns both English and Armenian literature and culture, without losing his heritage values, and [is] able to bring his contribution to the Armenian community."

\subsection{Additional Information on Family Characteristics}

Additional information on family characteristics is presented here to provide a more comprehensive picture of the families participating in this study. This information was not used in the statistical analyses of individual differences; however it is referred to in the discussion of the results. The majority of parents, i.e., $82.9 \%$ of fathers and $85.7 \%$ of mothers, reported Armenian as their first language. There were $73.2 \%$ of families, $73.7 \%$ in the $\mathrm{JK}$ and $72.7 \%$ in the SK, with both parents reporting Armenian as their first language. In addition, 9.8\% of fathers and $4.8 \%$ of mothers reported English as their first language. Finally, 7.3\% of fathers and 9.5\% of mothers reported other languages as their first language. Of the families (both father and mother), $43.2 \%$ were in Canada for less than 20 years, $58.8 \%$ for the JK and $30.0 \%$ for the SK children. Only $24.3 \%$ of the families (both father and mother) were in Canada for over 20 years, $17.6 \%$ for $\mathrm{JK}$ and $30.0 \%$ for $\mathrm{SK}$.

\subsubsection{Child Characteristics}

The two main child characteristics of interest in this study were nonverbal cognitive ability (nonverbal IQ) and phonological short-term memory (PSTM). The direct measurements used to assess these characteristics are described in the following two sections.

\subsection{Nonverbal Cognitive Ability}

Nonverbal cognitive ability (nonverbal IQ) has long been considered a correlate of vocabulary growth. Bloom (2001) argued that cognitive as well as linguistic abilities are involved in vocabulary learning, as previously discussed, based on a review of studies on word learning in children. 
The Brief IQ Screener from the Leiter International Performance Scale-Revised (Roid \& Miller, $1995,1997)$ for the age group of two to five years was used to measure nonverbal cognitive ability. This Screener was chosen for this study because all children, regardless of their language abilities in English, could easily understand and follow the instructions, which were largely nonverbal. This feature made the Screener suitable for the bilingual participants with varied proficiency in English.

The Screener encompassed four subtests, which were: Figure Ground, Form Completion, Repeated Patterns, and Sequential Order. The mean standard score for this test is 100 with a standard deviation of 15. The internal consistency reliability of this Screener is .88. The Screener correlates highly with the Wechsler Intelligence Scale for Children (WISC-III) Full Scale IQ and the WISC-III Performance IQ (Wechsler, 1991), $r=.85$ for both (Roid \& Miller, 1997).

The directions contained in the manual from the Screener were followed in test administration. The primary method suggested for communicating the instructions was pantomime. However, as described in the test manual, oral language could be used for participants with normal hearing. Therefore, when oral language was needed, the language most comfortably used by the participant at home (as reported on the parental questionnaire) was used. Occasional verbal feedback was used for encouragement.

During the screening the participants were asked to make arrangements with picture cards or rubber shapes or point to pictures on an easel, which held the visual stimuli. The easel was placed on a table and the investigator and the participant were seated across the corner of the table, i.e., at an angle of 90 degrees. This seating enabled the investigator to see the participant's pointing responses and the arrangements he/she made with the cards or shapes. The responses were recorded on a record form by the investigator.

To introduce the participant to this task, the investigator used the following script: We are going to play the last game for today. In this game we will play with picture cards and rubber shapes. I will give you the cards or shapes and you will put them in their places (while pointing to the slots on the easel). When you put a card or shape in its place I will mark it on the paper (while showing the record form on a clipboard) so that I will remember what your answers were. Now, we are going to practice some pictures together. 
As shown in Table 4, and as compared to the average range of the Screener scores, which is 85 115 , the JK children had a mean nonverbal IQ in the above average range and the SK children had a mean in the high average range. In addition, both groups showed SDs slightly smaller than the expected SD of the Screener. The wide range of scores of the SK children as compared to the restricted range of scores in $\mathrm{JK}$, with no scores below 100, might account for the significant difference observed between JK and SK, favouring the JK children. Further, the SK group included a possible outlier with a standard score of 83, i.e., slightly more than one standard deviation below the mean. Chapter 4 reports a supplementary analysis, which demonstrated that this possible outlier did not alter substantially the main statistical analyses.

\subsection{Phonological Short-Term Memory}

Phonological short-term memory is the second characteristic that has been shown to correlate with vocabulary growth in monolingual as well as bilingual children (e.g., Adams \& Gathercole, 1996, 2000; Service, 1992). To measure this characteristic the Hebrew Pseudo-word Phonological Short-Term Memory (PSTM) Task (Farnia \& Geva, 2011) was used. This task consisted of 27 pre-recorded 2- to 5-syllable pseudo-words (see Appendix H for task items). These pseudo-words follow the phonology of Hebrew, a language different from those spoken by the bilingual participants. Therefore, in theory, task performance should not be affected by familiarity with either English or Armenian languages. The choice of the Hebrew measure was also affected by the lack of a PSTM measure based on the phonology of the Armenian language. In the absence of such a measure, it was considered reasonable to use a measure that would, presumably, be unfamiliar to all participants, regardless of their varying levels of proficiency in Armenian and English languages. Unfamiliar though the Hebrew language might be to Armenian- or English-speakers, there is the possibility of some similarities between Hebrew phonology and the phonologies of the Armenian and English languages, which might affect performance in the Hebrew PSTM task used in this study. Nonetheless, these similarities were not investigated in the present study. Based on the nature of the correlations between growth rate in English vocabulary and English-like nonword tasks versus English vocabulary and Hebrewlike nonword tasks and the unique contributions of these tasks to the prediction of English receptive vocabulary, Farnia and Geva (2011) concluded that using a PSTM measure with pseudo-words based on an unfamiliar language can capture the relationship between PSTM and 
vocabulary more accurately than a PSTM measure with pseudo-words based on a familiar language.

The investigator introduced the participant to the Hebrew PSTM task by using the following script:

Now we are going to play the last game for today. We are going to listen to some new words on my computer. These words are not Armenian or English. When you hear a new word, you should repeat that word. I will record your voice when you say the new word. I will also write down your answers here (while showing the response form on a clipboard) so that I will remember what your answers were. Now we will practice some of these new words together.

The investigator's laptop computer provided the auditory stimuli for the PSTM task. During this task, the laptop was positioned on the table where the investigator and the participant were seated; however, it was facing the investigator and away from the participant to avoid visual distraction. The pre-recorded task was presented on the laptop computer at a normal intensity level that was comfortable to the ear and loud enough to hear easily. After playing the first practice trial, the investigator paused the laptop and waited for the participant's response. If there was no response the investigator asked the participant: What did you hear? Can you repeat it? If there was no response the investigator let the participant know that they were going to listen to the same trial again. The same trial was re-played. If again there was no response the investigator repeated the pseudo-word herself and encouraged the participant to repeat after her. Then, the participant was told that they were going to hear the second new word. This procedure continued until the participant repeated at least two of the four practice trials independently. To encourage the participant to continue with the task and to acknowledge his/her responses, encouraging feedback phrases such as Good; You are doing well; or That's fine were used occasionally. The main purpose of the use of encouraging feedback was to motivate the participant to continue with the task and to show his/her best performance while acknowledging his/her attention and cooperation during the task.

Once the four practice trials were given and the participant understood the procedures of the task, the investigator let the participant know that he/she would hear more new words and should repeat each new word immediately and completely. All task trials were presented even if there were consecutive incorrect responses or unanswered trials. Encouraging feedback was used 
occasionally during the tasks. Participants' responses were audio recorded. Meanwhile, the investigator scored the responses online using a response form. The complete repetition of each task trial was scored 1 . A repetition was considered complete if the child repeated the same number of syllables and phonemes with the exact order as that of the target pseudo-word. A task trial was scored 0 if it was repeated incompletely or was not repeated at all. Raw scores were then converted to percentage correct scores for each participant.

To obtain reliability for the scoring, the audio-recorded responses from $25 \%$ of the participants were re-scored independently by a second rater. The second rater was an adult Armenian-English bilingual with no familiarity with Hebrew. The rater had no prior familiarity with the task and was given the same instructions as followed by the researcher in scoring the responses. The results showed $91.8 \%$ agreement on the scoring of correct and incorrect responses.

As shown in Table 4, the SK children performed slightly better than the JK children on the PSTM task. However, the difference did not reach statistical significance. In addition, wide variability was observed among the participants in both grades in their PSTM scores, but this appeared to reflect task performance rather than other factors. All children were cooperative and showed no evident signs of lack of attention or fatigue during the task. Moreover, kindergarten participants in the present study showed higher scores as well as less variability than the grade one bilingual participants tested by Farnia and Geva (2011) with the same Hebrew PSTM measure. In that study, Hebrew scores were also consistently lower than those from an English PSTM measure, perhaps suggesting that language-specific measures tap language familiarity in addition to the ability to remember phonological information.

\subsection{Procedures}

The participants were tested individually by the investigator during four different sessions in a quiet room in their school. At each session, the investigator would take each participant from the classroom to a quiet room where the testing was performed. To greet the child the investigator would use the language most often used by the child, as indicated by the parents in the questionnaire. This language was also used for providing test instructions. To standardize test administration, the investigator followed clear and detailed instruction protocols and used relevant forms to keep a record of the responses. 
The tasks for each session were administered only after receiving participant assent for that session. The assent scripts for the four testing sessions or days are provided in Appendix $G$ in the order in which they were used. A positive answer to the first question on the script for each day was considered as the participant's assent to participate in the tasks for that day. At the end of each task the investigator would say: It's over, you did a great job, thank you. At the end of each testing session the participant had a choice of stickers, and then was taken back to his/her classroom.

\subsubsection{Timeline}

Each participant was tested twice over a six-month interval. The first testing (Time 1) was done in October - November 2008, two months after the start of the school year. The second testing (Time 2) was done in April - May 2009 towards the end of the school year (see Table 5 for a timetable). To counter the possible effect of fatigue at both times, each participant was tested on two separate days within a one-week period. However, at Time 2, four participants were tested within a two-week interval, because they were absent for several days during the data collection period.

As shown in Table 5, a fixed order of test administration was used for all participants. The decision was made to follow this order because the main focus was to elicit the best performance of the participants in the vocabulary tasks, which was the most important aspect of the study. Therefore, by following this fixed order the participants completed the vocabulary tasks first before other tasks.

On the first day (Day 1) at Time 1, the Armenian vocabulary tasks were given first followed by the Hebrew Pseudo-word PSTM Task. These tasks took between 30 and 45 minutes. On Day 2, the English vocabulary tasks were given first followed by the Brief IQ Screener. These tasks took 45 to 55 minutes. On the first day at Time 2 the Armenian vocabulary tasks were given. On the second day the English vocabulary tasks and a hearing screening test were given. Each day in the second time period took 20 to 35 minutes. To start the testing with a simpler task on all four days, the picture identification task in each language was administered first followed by the picture naming task in that language. Details on the administration of the vocabulary tasks and the hearing screening test will be provided in the following sections. 
Table 5.

Timetable for Task Administration at Time 1 and Time 2

\begin{tabular}{llc}
\hline Session & Task Order & Duration \\
\hline Time 1 & & \\
\hline Day 1 & 1. Armenian Picture Identification Task & $10-15 \mathrm{~min}$ \\
& 2. Armenian Picture Naming Task & $10-15 \mathrm{~min}$ \\
& 3. Hebrew Pseudo-word PSTM Task & $10-15 \mathrm{~min}$ \\
Day 2 & 1. English Picture Identification Task & $10-15 \mathrm{~min}$ \\
& 2. English Picture Naming Task & $10-15 \mathrm{~min}$ \\
& 3. Leiter-R: Brief IQ Screener & $25 \mathrm{~min}$ \\
\hline
\end{tabular}

Time 2

$\begin{array}{llr}\text { Day } 1 & \text { 1. Armenian Picture Identification Task } & 10-15 \mathrm{~min} \\ \text { 2. Armenian Picture Naming Task } & 10-15 \mathrm{~min} \\ \text { Day } 2 & \text { 1. English Picture Identification Task } & 10-15 \mathrm{~min} \\ & \text { 2. English Picture Naming Task } & 10-15 \mathrm{~min} \\ \text { 3. Otoacoustic Emission Test (hearing screening) } & 5 \mathrm{~min}\end{array}$




\subsubsection{Measures of Vocabulary}

\subsubsection{Settings and Apparatus}

As described in Chapter 2, a researcher-developed bilingual vocabulary test was used to measure Armenian and English picture identification and picture naming abilities. This test consisted of four vocabulary lists. A total number of 220 unrepeated target items were divided among the four lists. Therefore, each vocabulary list included 55 items. The four independent vocabulary lists were then used for the four vocabulary tasks: Armenian picture identification, Armenian picture naming, English picture identification, and English picture naming. The four vocabulary lists were counterbalanced across the four tasks according to a $4 \times 4$ Latin Square resulting in four different orders of the four lists, which were randomly assigned to participants in blocks of four. None of the target items for any individual child were repeated in the tasks at any given test time.

The investigator used a laptop computer, a microphone, and a response form for administering the vocabulary tasks and recording the responses. The visual stimuli for the vocabulary tasks were presented on a 14" Hewlett Packard Notebook display using the SuperLab Pro program (Cedrus, Version 2.0.4). The picture identification and the picture naming task trials (see Figures 1 and 2 for samples) were presented in the centre of the display one at a time. The SuperLab program was also used for electronic recording of the responses in all four tasks. In addition, a microphone was attached to the laptop for audio recording of participants' responses during the picture naming tasks. The Audacity program, a free digital audio editor (Sourceforge.net, Version 1.2.6) was used for this purpose. Furthermore, during all four tasks a hard copy of a response form, which contained the target items in all four tasks, was used to keep a record of responses. The investigator scored each target item as correct or incorrect on the form according to the participant's response.

During administration of the vocabulary tasks, the laptop computer was positioned on the table where the investigator and the participant were seated. It was placed in front of the participant so that the display was within his/her reach for pointing to the target pictures during the picture identification tasks. The investigator and the participant were seated across the corner of the table so that the investigator was to the left of the participant. This seating facilitated the use of two response keys on the laptop computer by the investigator, causing the least possible disturbance to the participant. 


\subsubsection{Picture Identification Tasks}

After receiving the participant's assent, the investigator described the procedure for the picture identification task using the following script:

Each time I will show you four pictures on this computer (while pointing to the laptop computer display). Then I will name one of the pictures in Armenian/English (depending on the task language of that day, i.e., Armenian on the first and English on the second) and will ask you to point to the picture. If you don't know the answer, try to guess and choose the one you think is best. I will also write down your answers here (while showing the response form on a clipboard) so that I will remember what your answers were. Now, we are going to practice some pictures together.

The investigator would then administer the practice trials. For the Armenian picture identification task, the first practice trial was shown and the participant was asked: Show me 'target picture' in Armenian. The same question was asked in English for the English picture identification task. During the practice trials, the investigator used one or two encouraging feedback phrases such as Good; You are doing well; or That's fine. The main purpose of the encouraging feedback was to motivate the participant to continue with the task and to show his/her best performance while acknowledging his/her attention and cooperation during the task.

In addition to encouraging feedback, corrective feedback was used during practice trials, if needed. This feedback was modeled after that recommended for administration of the PPVT-III (Dunn \& Dunn, 1997). Examples of corrective feedback were:

1. If the participant did not respond because he/she seemed not to understand what was expected, the investigator would say: I want you to point to 'target item', just like I $d o$. In the meantime the investigator would lightly touch the participant's hand and would direct him/her to point to the target picture.

2. If the participant did not know the correct response the investigator would encourage him/her to make a choice by guessing. Therefore the investigator would say: It's alright to guess; Try one; or Point to the one that you think it might be. 
3. If a participant responded very quickly or pointed to the same choice over and over again, and did not seem to consider all four options carefully, he/she was asked to: Slow down, don't hurry, look carefully at all pictures, and then choose the one you think is best. The investigator then would point to each picture in turn while saying: Look at this one, and this one, and this one, and this one; now point to 'target item'.

4. If a participant did not seem to listen carefully to the question, the investigator prompted him/her to do so by saying: Listen carefully.

After the practice trials, and once the investigator made sure that the participant knew what was expected from him/her, the task trials were administered. To start the task trials the following statement was used: Now I will show you other pictures. Then the task trials were presented one by one. For each task trial on the Armenian picture identification task the participant was asked in Armenian: Show me 'target picture' and the same question was asked in English for each task trial on the English picture identification task.

To keep the participant motivated and on task, encouraging feedback was provided during task trials. However, this feedback was not overused; it was used occasionally so that its occurrence was not predictable. In addition, it was used equally for correct or incorrect responses to acknowledge the participant's attention and his/her cooperation during the task. No corrective feedback was used for task trials.

Each correct response was scored 1 and each incorrect response was scored 0 . The unanswered trials were scored 0 . The total score in each task ranged from 0 to 55. A stopping rule was applied in a similar manner to most vocabulary tests. If there were five consecutive incorrect responses or unanswered trials the testing was stopped to prevent frustration.

\subsubsection{Picture Naming Tasks}

After finishing the identification task in one language, the naming task in that language was administered. The procedure for the picture naming task was described to each participant using the following script:

Each time I will show you a picture on this computer (pointing to the laptop computer display) and will ask you 'what's this?' (in Armenian for the Armenian task and in English for the English task). Then you should name the picture in Armenian/English (depending on the task 
language of the day, i.e., Armenian on the first and English on the second day). If you don't know the name of that picture in Armenian/English (depending on the task language), try to guess and say one you think is best. I will record your voice when you name the pictures. I will also write down your answers here (while showing the response form on a clipboard) so that I will remember what your answers were. Now, we will practice some pictures together.

The encouraging feedback used during practice trials in the naming tasks was the same as that used in the practice trials in the identification tasks. However, because the task at hand was naming, the corrective feedback was revised accordingly. The revised feedback was:

1. If the participant did not respond because he/she seemed not to understand what was expected, the investigator would say: I want you to name this picture (while pointing to the picture on the display), just like I do. Then the investigator would name the picture herself and would ask the participant: What would you name it?

2. If the participant did not know the correct response the investigator would encourage him/her to respond by guessing. Therefore the investigator would say: It's alright to guess; or Say the word you think it is.

3. If a participant responded very quickly or used the same word for naming different pictures, he/she was asked to: Slow down, don't hurry; or Look carefully at the picture, and then name it.

4. If a participant did not seem to listen carefully to the question, the investigator prompted him/her to do so by saying: Listen carefully.

After the practice trials and once the investigator made sure that the participant knew what was expected from him/her, the task trials were administered by saying to the participant: Now, I will show you other pictures. Then the task trials were presented one by one. For each task trial in the Armenian picture naming task the child was asked the question: What's this? in Armenian and for the trials in the English picture naming task the same question in English was asked. The encouraging feedback, as discussed earlier, was used occasionally. No corrective feedback was used during task trials. 
Each correct response was scored 1 and each incorrect response was scored 0 . An incorrect response was a label given to a picture other than the expected labels used by the adults in the Labelling and Age of Acquisition Rating Task (see Section 2.2.1, for details). A correct response was one clearly related to the target word in number of syllables and phonemes with intelligible pronunciation. Any unanswered trials were also scored 0 . The total score in each task ranged from 0 to 55. For the same reason as discussed in the identification tasks, the testing was stopped if there were five consecutive incorrect responses or unanswered trials.

Reliability was obtained for the scoring of Armenian and English picture naming tasks. The audio-recorded responses from a randomly selected sample of $25 \%$ of the participants in Armenian and English picture naming tasks at Time 1 and Time 2 were re-scored independently by a second rater. The second rater was an adult Armenian-English bilingual with no prior familiarity with the tasks. The rater was given the same instructions as followed by the researcher in scoring the responses. The results showed $99.6 \%$ agreement on the scoring of correct and incorrect responses in the Armenian picture naming task and $96.3 \%$ agreement on the scoring of correct and incorrect responses in the English picture naming task.

\subsubsection{Additional Measures}

Information was also obtained on the language development and hearing abilities of the participants. This information was supplementary and was used for understanding vocabulary task performance in participants.

\subsubsection{Language Development}

Information was obtained from parents at Time 1 regarding any concerns they had about their child's language development. This information was obtained from answers provided by parents to five questions in 'Home Language Use Questionnaire' (Appendix F, questions 20 to 23). The answers to these questions provided some information on the language development of the participants. This information was important because concerns about language development could have implications for vocabulary task performance. The results, however, showed that none of the parents had any major concerns about their child's language development. 


\subsubsection{Hearing Abilities}

The participants were screened for their hearing abilities at Time 2. The result of this screening was considered as an estimate of whether the participants had normal hearing at the time of study, because hearing problems could interfere with their performance in the vocabulary as well as the PSTM tasks. A decision was made to include this screening together with two questions forwarded to the parents at Time 2 (see Appendix G, Consent Form - Time 2). In these questions the parents were asked retrospectively about the hearing status of their child during the course of the study. These questions concerned the existence and nature of any hearing problems or ear infections in the last eight months, i.e., two months before the start of the study at Time 1 . The results showed no problems or concerns regarding the hearing abilities of the children.

The Otoacoustic Emission (OAE) test was used to screen the participants for their hearing abilities. This test was appropriate for use in this study because it took a very short time to administer and required no verbal or motor responses from the participants. This screening was administered on the second day at Time 2 using the Vivolink portable device (Vivosonic Integrity, Version 4.01). During the screening the participant was seated on a chair. The investigator placed a small probe in his/her ear. The automatic responses of each ear to a sound presented through a small microphone were recorded and monitored on the screen of the laptop computer connected to the device. This screening took about five minutes to administer. The program provided the results of the screening on a "Pass", "Fail", or "Refer" basis.

The investigator received parental consent for the hearing screening for 41 participants. Parental consent was not received for one participant who was already on medication because of a unilateral ear infection. The vocabulary and PSTM task results of this particular participant were above the average, suggesting limited interference of possible hearing problems on task performance. All tested participants passed the screening.

\subsubsection{Program Fidelity}

A systematic observation scheme was developed to verify the balanced use and instruction of the Armenian and English languages in their respective classes in the two schools. The two schools participating in the study were providing a bilingual program where both Armenian and English were supposed to be used and instructed under equal conditions and in a balanced manner. 
Therefore, an observation scheme following Eilers, Oller, and Cobo-Lewis (2002) was used. The purpose of this observation was to measure the frequency of use of English in Armenian classes and that of Armenian in English classes. For this purpose, the investigator observed each JK and SK classroom in both schools $(n=6)$ four times, at two-month intervals, during the academic year after receiving the teachers' informed consent (see Appendix I). The use of four periods of observation provided a general overview of the use of the languages throughout the year.

The students in each class had two separate half-day language sessions per day, one in Armenian and the other in English. Each language session was taught by a separate teacher. At each observation time the investigator visited each class twice, once for the Armenian and the other for the English session. During the observation the investigator sat at the rear of the classroom. Each observation session lasted half-an-hour. This amount of time was considered adequate based on the teacher-directed nature of the instruction administered in these classes. However, arrangements were made in advance with teachers to make sure that the investigator would observe the classes during instructional periods. This helped to observe instances where teachers were talking to the class.

Each half-hour observation session was divided into six 5-minute observation blocks. During the second and fourth minute of each block the investigator used the Classroom Observation Checklist (see Appendix J), designed for this observation, to keep a record of the instances of use of the language other than the language of instruction by the teacher to the class or to a student. One checklist was used for each class. Page 1 was used for recording instances of use of English language during the Armenian session, and Page 2 was used for recording instances of use of Armenian language during the English session. The instances of use of these languages were tallied on the checklist. These instances included single words, phrases, or sentences. A stopwatch was used to keep track of the timing blocks. At the end, the total number of uses of the language other than the language of instruction by the teacher was calculated for each language session.

The results showed that with the exception of one session, the language intended for that session was used exclusively. The only exception was in one of the Armenian sessions where the Armenian teacher was describing the Halloween tradition. During this session, four instances of the use of English terms were observed. These terms were used as translation equivalents. As a 
general conclusion, the observation verified the balanced nature of the bilingual program in both schools. However, interestingly enough, in informal observations during the visits to the school for data collection, the researcher noticed that English was the predominant language used by the children in the hallways. 


\section{Results}

This study of bilingual vocabulary pursued two objectives. These objectives addressed group and individual differences in vocabulary in bilingual children attending Junior Kindergarten (JK) and Senior Kindergarten (SK). To investigate group differences in vocabulary growth, Armenian and English picture identification and picture naming task performances were compared at two times of testing over a six-month interval in two groups of JK and SK children. To examine individual differences the best predictors of Armenian and English vocabulary performance were identified.

Two sets of data were collected. The first set included data on Armenian and English vocabulary task performance collected from all participants in JK and SK at Time 1 and Time 2 over a sixmonth interval. The second set included data on environmental and child characteristics collected at Time 1 from all participants. To analyze the two sets of data, mixed analysis of variance (ANOVA) and multiple hierarchical regression analyses were used, respectively. Mixed ANOVA involves two types of variables, i.e., at least one between-subjects variable and at least one within-subjects variable. These variables are defined in Section 4.4.1.3. In addition, post-hoc analyses were also used, which included mixed analysis of covariance (ANCOVA), paired samples $t$-tests, and independent samples $t$-tests. An alpha level of $p<.05$ was used in all analyses. The first hypothesis-testing analysis dealt with group differences and the second dealt with individual differences.

Before presenting the results of the statistical analyses mentioned above, three topics are covered. First the results of a preliminary analysis are presented. Next the validity and reliability of the vocabulary test are described. In the final section the results of a power analysis are provided.

\subsection{Preliminary Analysis on Possible Effects of List Order}

A preliminary analysis was performed to examine the potential effect of Order, i.e., order of the four lists, on vocabulary task performance. As described in Chapter 2, four lists of vocabulary items were counterbalanced across the four vocabulary tasks. Hence there were four different list orders randomly assigned to participants in blocks of four. The use of different orders of the four lists for the four tasks raised a concern over the potential effect of order on vocabulary task performance. To address this concern a preliminary analysis was performed. 
In the preliminary analysis, Order (1, 2, 3, and 4) was included as a between-subjects variable, in addition to four other main variables of interest (Grade [between-subjects], Language, Modality, and Time [within-subjects]). The analysis showed no significant main effects or interactions involving Order. Based on the results of this preliminary analysis, only the other four variables were included in the subsequent hypothesis-testing analyses.

\subsection{Vocabulary Test Validity and Reliability}

As mentioned in Chapter 1, this dissertation project encompassed two separate studies. Study 1 involved developing a parallel bilingual vocabulary test. This test was intended to be equally applicable in assessing Armenian and English receptive and expressive vocabularies in a longitudinal study, Study 2, so that vocabulary growth in both languages could be captured reliably over the course of six months.

As described in Chapter 2, this vocabulary test was developed using a rigorous procedure based on age of acquisition (AoA) ratings obtained from adult Armenian (Western and Eastern dialects) and English speakers familiar with language development in children. The mean AoA value obtained from these three language groups was used as a measure for ordering the vocabulary items from easy to difficult and for developing four parallel lists (A, B, C, and D). These lists were then counterbalanced across the four Armenian and English picture identification and picture naming tasks.

As previously described, the decision to use adult speakers as the source for AoA ratings was based on the assumption that their familiarity with child language development could provide them with the ability to make sound decisions on the age that children learn a vocabulary item in their language. In addition, as discussed in Chapter 2, previous studies (e.g., Morrison et al., 1992) have found significant correlations between subjective ratings and objective AoA values obtained from picture naming performances of children.

To further test this assumption, an item analysis was performed on the vocabulary test. The main purpose of this analysis was to validate the use of AoA ratings in developing the vocabulary lists. Therefore, simple correlations were computed between the subjective AoA ratings of the items by the adults and the percentage of child participants providing correct responses to the items. The correlation analyses showed significant negative Pearson correlation coefficients between: 
(a) the subjective mean AoA ratings obtained from adults in all three language groups (Western Armenian, Eastern Armenian, and English) and the percentages of child participants with correct responses to the items in all vocabulary tasks, $r(200)=-.880, p<.001$; (b) the subjective mean AoA ratings obtained from adult Armenian speakers (Western and Eastern Armenian) and the percentage of child participants with correct responses to the items in Armenian vocabulary tasks, $r(200)=-.890, p<.001$; and (c) the subjective mean AoA ratings obtained from adult English speakers and the percentage of child participants with correct responses to the items in English vocabulary tasks, $r(200)=-.851, p<.001$. It should be noted that the degrees of freedom in these correlations were 200, because AoA subjective ratings were available for 200 items. As discussed in Chapter 2, originally only 200 items from the pool of 343 items used in Labelling and Age of Acquisition Rating task were chosen as targets. The additional 20 target items were added later, after the pilot study, from English AoA studies or standard measures. Therefore, the AoA values for these items were obtained from their respective sources. The high correlations across items between the subjective AoA ratings and the percentage of correct vocabulary responses validated the use of subjective AoA ratings from the adult speakers of the three languages in selecting the vocabulary items and ordering them from easy to difficult.

Correlation analyses also were performed to address the test-retest reliability of the vocabulary test in assessing the performance of participants at two separate times, six months apart. In these analyses, the correlations were computed on the mean percentages of participants' correct responses to the items in the vocabulary tasks at Time 1 and Time 2 . The results showed high significant Pearson correlation coefficients between the percentage of correct responses to the vocabulary tasks at Time 1 and Time $2, r(42)=.893, p<.001$. There were also high significant

correlations between the percentage of correct responses to Armenian vocabulary tasks at Time 1 and Time $2, r(42)=.859, p<.001$, and the percentage of correct responses to English vocabulary tasks at Time 1 and Time $2, r(42)=.901, p<.001$. These results suggest that the use of the vocabulary test at two different times provided stable and reliable results across participants.

\subsection{Power Analysis}

In designing this study, a power analysis was performed as previously described. The estimated power to detect a large effect $(d=.80)$ was .70 for within-group vocabulary growth and greater 
than .90 for full-sample vocabulary growth, which was calculated based on an assumed correlation of .50 between Time 1 and Time 2 scores. Results of the study showed a correlation of .89 between Time 1 and Time 2 scores. Therefore, the actual power to detect a large effect for within-group vocabulary growth was .99 (Portney \& Watkins, 2000, p. 708).

\subsection{Hypothesis-Testing Analyses}

\subsubsection{Group Differences in Bilingual Vocabulary Performance}

\subsubsection{Hypotheses}

The first and main objective of the study involved comparisons of vocabulary task performance in children from JK and SK grades at two times of testing. Percent correct scores were obtained from the JK and SK children after administering four vocabulary tasks, i.e., Armenian picture identification, Armenian picture naming, English picture identification, and English picture naming at Time 1 and 2 .

To reiterate, it was hypothesized that there would be main effects of Grade, Time, Modality, and Language and a significant Language by Time interaction. Therefore the main predictions of interest were: (1) Children in SK will show higher vocabulary performance than children in JK; (2) Vocabulary performance at Time 2 will be higher than vocabulary performance at Time 1;

(3) Performance in picture identification will be higher than performance in picture naming; (4) Performance in Armenian will be higher than performance in English; and (5) The rate of vocabulary growth in English will be greater than the rate of growth in Armenian.

The first three hypotheses were based on general developmental trends regarding vocabulary observed in young monolingual as well as bilingual children. The last two were based on the assumption that children in this study would generally come from families with Armenian as the predominant language in the home; therefore they would be primarily more proficient in Armenian than English. Upon entering the bilingual program, these children would show a higher rate of growth in English, due to increased exposure to English in the school setting as compared to the home. 


\subsubsection{Descriptive Statistics on Vocabulary Performance}

Table 6 presents means and standard deviations for percent correct scores in the two grades (JK and SK) at Time 1 and Time 2 in the four individual tasks of Armenian picture identification, Armenian picture naming, English picture identification, and English picture naming, as well as for the Armenian and English vocabulary tasks (picture identification and picture naming combined). This table also includes the descriptive statistics for the bilingual vocabulary test (all four tasks combined). As shown in Table 6, the scores in all four vocabulary tasks increased between Time 1 and Time 2 in the two grades.

The smaller standard deviations in the English picture identification task in the SK children as compared to the other conditions were indicative of possible ceiling effects. At Time 1 there were two participants who scored between $90-93 \%$ on this task and at Time 2 there were five more participants in addition to these two, who scored over $90 \%$. Three of these participants scored over $95 \%$. 


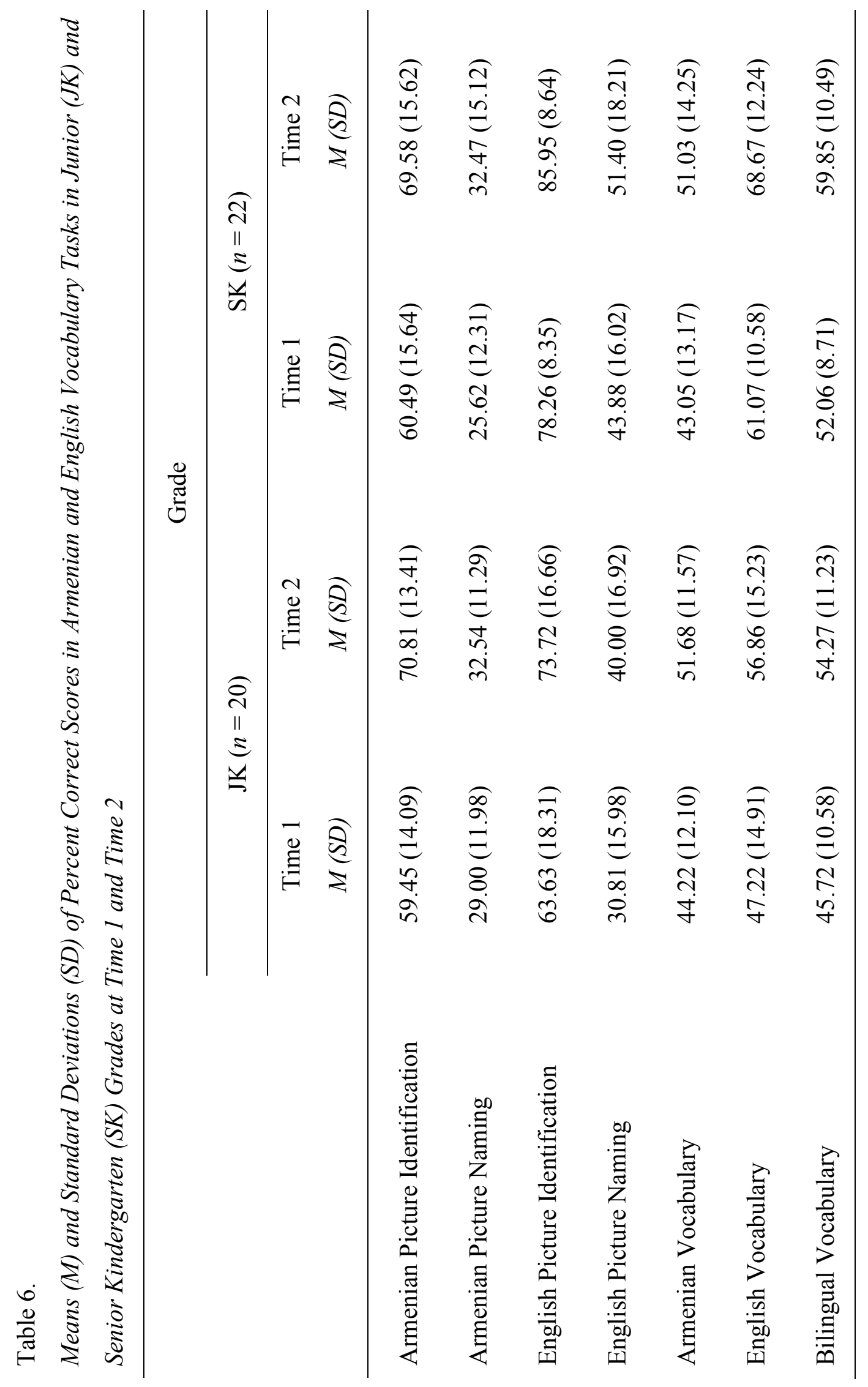




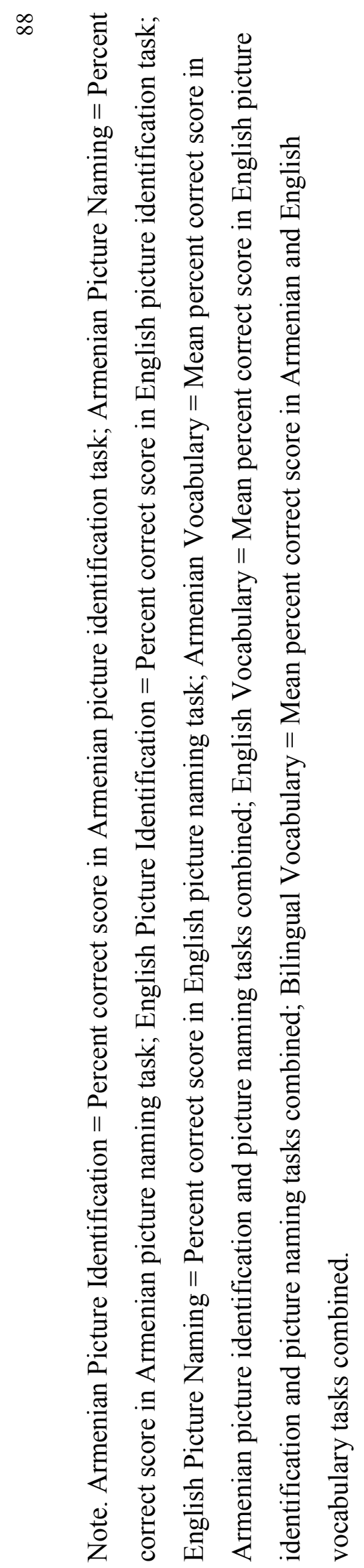




\subsubsection{Mixed Analysis of Variance of Group Differences in Vocabulary Performance}

Mixed ANOVA was used to examine the hypotheses regarding group differences in Armenian and English receptive and expressive vocabulary growth from Time 1 to Time 2 in the JK and SK grades. The between-subjects variable was Grade, with two levels of JK and SK. The withinsubjects variables were Time with two levels of Time 1 and Time 2, Modality with two levels of picture identification and picture naming, and Language with two levels of Armenian and English.

As presented in Table 7, the results showed two significant interactions and three significant main effects. There were interactions of Language by Modality by Time, $F(1,40)=5.319, p=$ $.026, \eta^{2}=.117$ and Language by Grade, $F(1,40)=8.123, p=.007, \eta^{2}=.169$. In addition, there were main effects of Time, $F(1,40)=109.730, p<.001, \eta^{2}=.733$; Modality, $F(1,40)=$ $668.654, p<.001, \eta^{2}=.944$; and Language, $F(1,40)=20.677, p<.001, \eta^{2}=.341$.

The data on nonverbal IQ scores of the SK children, presented in Table 4, showed a wide range of scores with one participant scoring more than one standard deviation below the mean. To make sure that this participant was not affecting the results, a second mixed ANOVA was conducted with this participant excluded. The results remained the same, showing the same main effects and interactions as outlined above. Therefore, the results reported here include this participant. 
Table 7.

Mixed Analysis of Variance of Between- and Within-Subjects Variables on Vocabulary Task Performance

\begin{tabular}{|c|c|c|c|c|c|c|}
\hline Source of Variance & $S S$ & $M S$ & $d f$ & $F$ & $p$ & $\eta^{2}$ \\
\hline \multicolumn{7}{|l|}{ Between-Subjects } \\
\hline Grade & 2977.791 & 2977.791 & 1 & 3.756 & .060 & .086 \\
\hline Error & 31709.035 & 792.726 & 40 & & & \\
\hline \multicolumn{7}{|l|}{ Within-Subjects } \\
\hline Language & 10068.701 & 10068.701 & 1 & 20.677 & $<.001$ & .341 \\
\hline Language*Grade & 3955.359 & 3955.359 & 1 & 8.123 & .007 & .169 \\
\hline Error (Language) & 19477.630 & 486.941 & 40 & & & \\
\hline Modality & 99885.758 & 99885.758 & 1 & 668.654 & $<.001$ & .944 \\
\hline Modality*Grade & 41.602 & 41.602 & 1 & .278 & .601 & .007 \\
\hline Error (Modality) & 5975.331 & 149.383 & 40 & & & \\
\hline Time & 5590.572 & 5590.572 & 1 & 109.730 & $<.001$ & .733 \\
\hline Time*Grade & 11.981 & 11.981 & 1 & .235 & .630 & .006 \\
\hline Error (Time) & 2037.930 & 50.948 & 40 & & & \\
\hline Language*Modality & 35.952 & 35.952 & 1 & .317 & .577 & .008 \\
\hline Language*Modality*Grade & 1.005 & 1.005 & 1 & .009 & .926 & .000 \\
\hline Error (Language*Modality) & 4542.964 & 113.574 & 40 & & & \\
\hline Language*Time & 17.159 & 17.159 & 1 & .447 & .508 & .011 \\
\hline Language*Time*Grade & 34.160 & 34.160 & 1 & .890 & .351 & .022 \\
\hline Error (Language*Time) & 1534.760 & 38.369 & 40 & & & \\
\hline Modality*Time & 162.044 & 162.044 & 1 & 3.460 & .070 & .080 \\
\hline Modality*Time*Grade & 52.481 & 52.481 & 1 & 1.120 & .296 & .027 \\
\hline Error (Modality*Time) & 1873.542 & 46.839 & 40 & & & \\
\hline Language*Modality*Time & 105.488 & 105.488 & 1 & 5.319 & .026 & .117 \\
\hline Language*Modality*Time*Grade & 30.714 & 30.714 & 1 & 1.549 & .221 & .037 \\
\hline Error (Language*Modality*Time) & 793.302 & 19.833 & 40 & & & \\
\hline
\end{tabular}


Figure 3 illustrates the 3-way interaction of Language by Modality by Time (collapsed across Grade) found in the main analysis. The panels depict the Modality by Time interaction in Armenian (left) and English (right). As shown, the 2-way Modality by Time interactions showed different patterns in the two languages. Post-hoc tests showed that the 2-way interaction was significant in Armenian, $F(1,41)=7.605, p=.009, \eta^{2}=.156$, but not significant in English, $F$ $(1,41)=.083, p=.774, \eta^{2}=.002$. As shown in the left panel, the pattern of growth in Armenian picture identification was different than the pattern of growth in Armenian picture naming. Posthoc paired samples $t$-tests showed that growth in Armenian picture identification, $(M=10.17 \%$, $S D=10.03 \%)$, was significantly greater than growth in Armenian picture naming, $(M=5.28 \%$, $S D=7.53 \%) t(41)=2.758, p=.009$, whereas growth in English picture identification, $(M=$ $8.83 \%, S D=9.25 \%)$, was statistically similar to growth in English picture naming, $(M=8.31 \%$, $S D=8.21 \%) t(41)=0.289, p=.774$.

In addition to the 3-way interaction, Figure 3 illustrates the main effects of Time, Modality, and Language. It was predicted that performance at Time 2 would be higher than performance at Time 1. The Time effect (collapsed across Grade, Language, and Modality) supported this prediction and indicated that performance at Time $2(M=57.06 \%, S D=11.08 \%)$ was significantly better than performance at Time $1(M=48.89 \%, S D=10.05 \%)$. In addition, it was predicted that performance in picture identification would be higher than performance in picture naming. The Modality effect (collapsed across Grade, Language, and Time) supported this prediction and indicated that performance in picture identification $(M=70.24 \%, S D=10.40 \%)$ was significantly better than performance in picture naming $(M=35.71 \%, S D=11.83 \%)$. Finally, it was predicted that performance in Armenian vocabulary would be higher than performance in English vocabulary. However, the Language effect (collapsed across Grade, Modality, and Time) did not support this prediction. Contrary to the prediction, this main effect indicated that performance in English vocabulary $(M=58.46 \%, S D=14.28 \%)$ was significantly better than performance in Armenian vocabulary $(M=47.50 \%, S D=12.25 \%)$. 


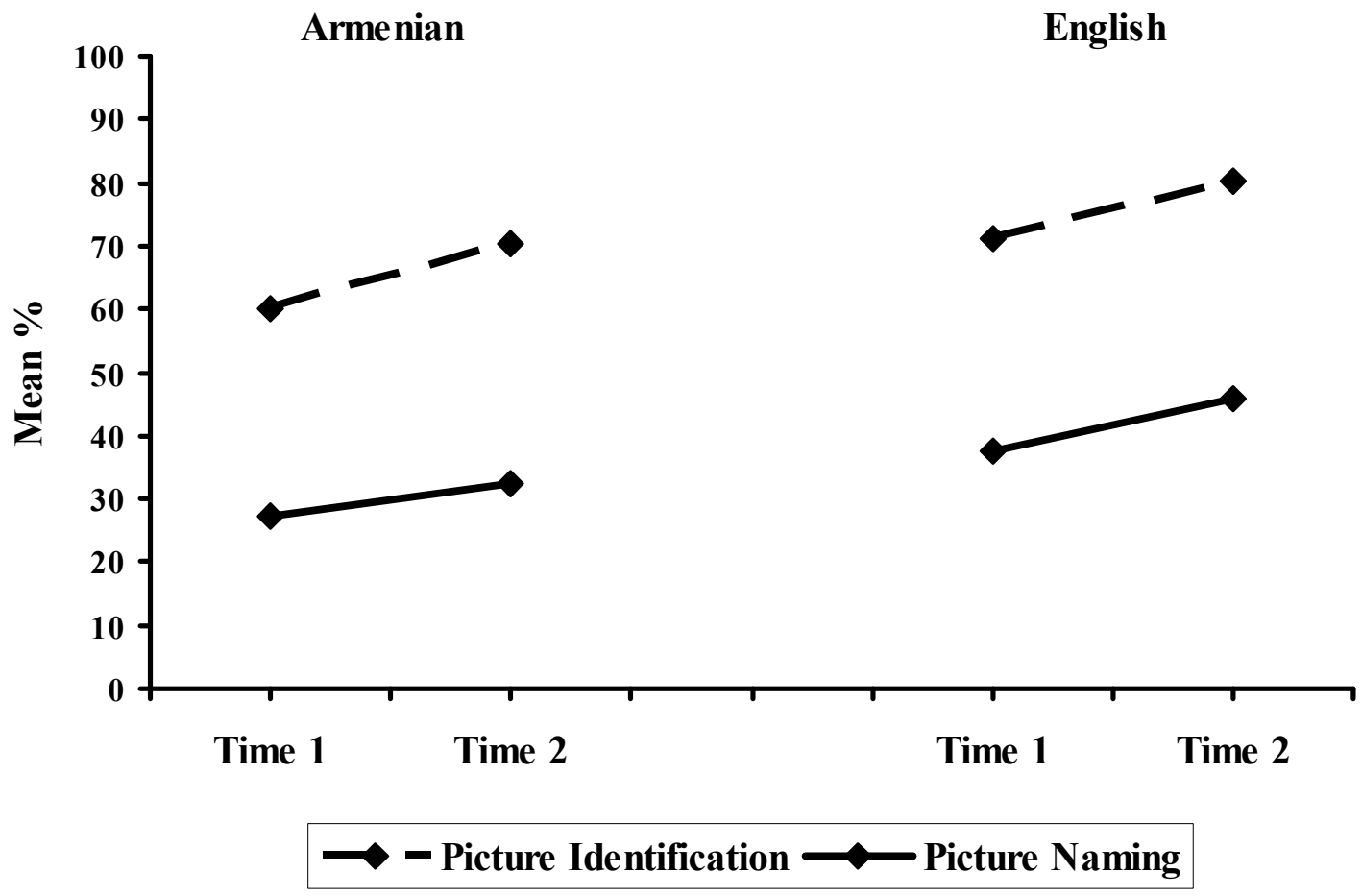

Figure 3. Significant 3-way interaction of Language by Modality by Time, collapsed across Grade, with lines representing mean percent correct scores in Armenian (left panel) and English (right panel) picture identification and picture naming tasks at Time 1 and Time 2. 
The 2-way interaction of Language by Grade (collapsed across Modality and Time) is illustrated in Figure 4. As depicted, the SK children compared to the JK children showed better performance in English tasks than in Armenian. As post-hoc tests, two independent samples $t$ tests were used to compare the differences in Armenian and English task performance between the JK and SK grades. There was no significant difference between the JK $(M=47.95 \%, S D=$ $11.55 \%)$ and SK children $(M=47.04 \%, S D=13.12 \%)$ in Armenian tasks $t(40)=.237, p=.814$. However, the SK children $(M=64.87 \%, S D=11.13 \%)$ performed significantly better than the JK children $(M=52.04 \%, S D=14.57 \%)$ in English tasks $t(40)=-3.223, p=.003$. In other words, language had a differential effect on the vocabulary performance of children in each grade.

Two other hypotheses were not supported. Inconsistent with the Grade effect hypothesis, which predicted higher performance in the SK children compared to the JK children, the results showed no significant difference between the JK and SK children in their overall vocabulary performance, $F(1,40)=3.756, p=.060, \eta^{2}=.086$. However, this difference approached significance and was in the expected direction, i.e., higher performance by SK than by JK children. The data also did not support the hypothesized Language by Time interaction hypothesis, $F(1,40)=.447, p=.508, \eta^{2}=.011$, which predicted higher growth in English compared to Armenian vocabulary. 


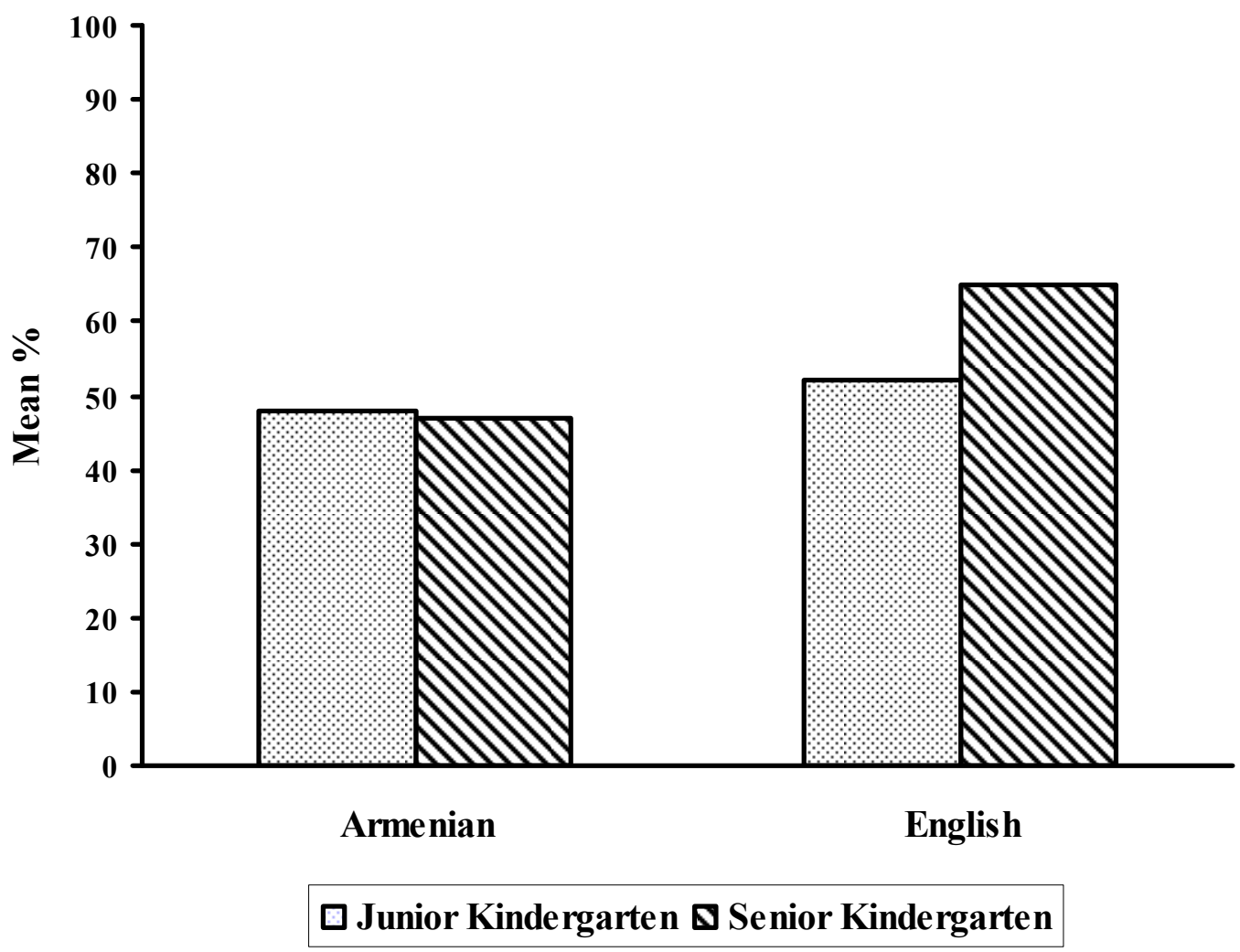

Figure 4. Significant 2-way interaction of Language by Grade, collapsed across Modality and Time, with bars representing mean percent correct scores in Armenian and English vocabulary tasks in Junior Kindergarten (JK) and Senior Kindergarten (SK) children. 


\subsubsection{Supplementary Analyses of Group Differences in Vocabulary Performance}

\subsection{Mixed Analysis of Covariance}

The ANOVA findings, combined with information on English language exposure at home, suggested that there might be a potential confound that affected task performance in the two languages. The Language by Grade interaction indicated different patterns of Armenian and English vocabulary in the two grades. The SK children performed significantly better than the JK children in English tasks, but there were no significant differences between the two grades in Armenian tasks. Furthermore, as shown in Table 4, the SK children had higher English language exposure at home than the JK children. This grade difference in language exposure might account for the observed pattern. Therefore, as a post-hoc analysis, mixed ANCOVA was performed on vocabulary task performance with English language exposure as a covariate. This analysis was intended to examine whether the difference in English language exposure between the JK and SK grades could account, at least in part, for the different patterns of Armenian and English vocabulary performance in the two grades.

In the mixed ANCOVA, the same variables were used as those in mixed ANOVA with the exception that English language exposure in the home was included as a covariate. As a result, the main effects and interactions of the between- and within-subjects variables were examined while partitioning out the variability that could be attributed to the covariate. It was predicted that English language exposure might account, at least in part, for the Language by Grade interaction and the Language main effect observed in the original ANOVA.

As presented in Table 8, the ANCOVA results showed one significant interaction and three significant main effects, once differences in English language exposure were taken into account. There was a significant 4-way interaction of Language by Modality by Time by Grade, $F(1,39)$ $=5.765, p=.021, \eta^{2}=.129$. Contrary to the ANOVA results, the ANCOVA showed a significant main effect of Grade, $F(1,39)=4.252, p=.046, \eta^{2}=.098$, which supported the ANCOVA, as well as the original ANOVA prediction of higher performance in the SK children compared to the JK children. As in the ANOVA analysis and consistent with the predictions for the ANCOVA and ANOVA, the ANCOVA also showed significant main effects of Time, $F$ (1, $39)=56.051, p<.001, \eta^{2}=.590$; and Modality, $F(1,39)=366.866, p<.001, \eta^{2}=.904$. In what 
follows, these results, based on adjusted means, are presented. The findings on the Language by Grade interaction and Language main effect are presented first, followed by other results.

As mentioned earlier, the initial ANOVA showed a significant Language by Grade interaction, in which the SK children showed better performance than the JK children in English, but not in Armenian. However, as revealed by the ANCOVA, when the two grades were equated on English language exposure at home, this interaction was no longer significant. Although the Language by Grade interaction in the ANCOVA did not reach significance, the $p$ value of .070 approached significance and the pattern of adjusted means was similar to that observed in the ANOVA.

Unlike the findings in the ANOVA showing higher performance in English compared to Armenian, the Language main effect in the ANCOVA was not significant, $F(1,39)=.231, p=$ $.633, \eta^{2}=.006$. Inconsistent with the original prediction of higher performance in Armenian compared to English, this finding indicated that, once the JK and SK grades were equated statistically on English language exposure at home, their Armenian and English vocabulary performances were similar.

The ANCOVA showed a 4-way Language by Modality by Time by Grade interaction. This 4way interaction is illustrated in Figure 5. The panels depict the 3-way interactions of Modality by Time by Grade in Armenian (left) and English (right). As shown in Figure 5, the 3-way interactions showed different patterns in the two languages. Post-hoc tests showed that the 3-way interaction was significant in Armenian, $F(1,39)=5.910, p=.020, \eta^{2}=.132$, but not significant in English, $F(1,39)=.010, p=.923, \eta^{2}=.000$. As depicted in the left panel in Figure 5, Armenian picture identification and picture naming showed different patterns of growth for JK (presented in blue) and SK (presented in red) children. Additional post-hoc tests showed that the 2-way interaction of Modality by Time was significant in $\mathrm{JK}, F(1,18)=7.246, p=.015, \eta^{2}=$ .287 , but not significant in SK, $F(1,20)=2.371, p=.139, \eta^{2}=.106$. This significant interaction indicated that the JK children showed less growth in the Armenian picture naming task than in the Armenian picture identification task. This finding was not part of the original hypotheses and predictions of the study.

A 4-way interaction in the presence of a covariate is a challenge to interpret. However, there were some similarities between the ANCOVA finding regarding the 4-way interaction of 
Language by Modality by Time by Grade and the ANOVA finding regarding the 3-way interaction of Language by Modality by Time (collapsed across Grade). These findings both showed different patterns of Modality growth in Armenian and English. Overall, picture naming showed less growth than picture identification in Armenian, whereas growth in these two modalities was similar in English. However, based on the ANCOVA finding this difference in Modality growth pattern was present only in the JK children. In other words, in the ANOVA the difference between Armenian picture identification and picture naming growth was present across both JK and SK grades, whereas in the ANCOVA, as a result of the statistical adjustments of English language exposure, this difference was only observed in the JK but not the SK.

In addition to the 4-way interaction, Figure 5 illustrates the main effects of Grade, Time, and Modality. The findings on these three main effects described below were consistent with the initial ANOVA predictions of higher vocabulary performance in SK compared to JK, at Time 2 compared to Time 1, and in picture identification compared to picture naming. The Grade effect (collapsed across Language, Modality, and Time) indicated that children in the SK grade showed significantly higher performance in overall vocabulary $\left(M_{a d j}=56.31 \%, S D=10.25 \%\right)$ than children in the JK grade $\left(M_{a d j}=49.61 \%, S D=10.28 \%\right)$. As illustrated in Figure 5, the Time effect (collapsed across Grade, Language, and Modality) indicated that performance at Time 2 $\left(M_{a d j}=57.04 \%, S D=10.94 \%\right)$, was significantly better than performance at Time $1,\left(M_{a d j}=\right.$ $48.87 \%, S D=9.70 \%$ ). Finally, the Modality effect (collapsed across Grade, Language, and Time $)$ indicated that performance in picture identification $\left(M_{a d j}=70.21 \%, S D=9.97 \%\right)$ was significantly better than performance in picture naming $\left(M_{a d j}=35.70 \%, S D=11.81 \%\right)$. However, as was the case in the ANOVA, the ANCOVA findings did not support the hypothesized Language by Time interaction, $F(1,39)=.988, p=.326, \eta^{2}=.025$. 
Table 8 .

Mixed Analysis of Covariance of Between- and Within-Subjects Variables on Vocabulary Task Performance, with English Language Exposure at Home as the Covariate

\begin{tabular}{|c|c|c|c|c|c|c|}
\hline Source of Variance & $S S$ & $M S$ & $d f$ & $F$ & $p$ & $\eta^{2}$ \\
\hline \multicolumn{7}{|l|}{ Between-Subjects } \\
\hline Grade & 3408.962 & 3408.962 & 1 & 4.252 & .046 & .098 \\
\hline Error & 31268.001 & 801.744 & 39 & & & \\
\hline \multicolumn{7}{|l|}{ Within-Subjects } \\
\hline Language & 61.323 & 61.323 & 1 & .231 & .633 & .006 \\
\hline Language*Grade & 920.806 & 920.806 & 1 & 3.470 & .070 & .082 \\
\hline Error (Language) & 10348.016 & 265.334 & 39 & & & \\
\hline Modality & 55635.600 & 55635.600 & 1 & 366.866 & $<.001$ & .904 \\
\hline Modality*Grade & 72.994 & 72.994 & 1 & .481 & .492 & .012 \\
\hline Error (Modality) & 5914.393 & 151.651 & 39 & & & \\
\hline Time & 2928.390 & 2928.390 & 1 & 56.051 & $<.001$ & .590 \\
\hline Time*Grade & 12.138 & 12.138 & 1 & .232 & .632 & .006 \\
\hline Error (Time) & 2037.545 & 52.245 & 39 & & & \\
\hline Language*Modality & 59.559 & 59.559 & 1 & .514 & .478 & .013 \\
\hline Language*Modality*Grade & 6.060 & 6.060 & 1 & .052 & .820 & .001 \\
\hline Error (Language*Modality) & 4519.069 & 115.874 & 39 & & & \\
\hline Language*Time & 38.340 & 38.340 & 1 & .988 & .326 & .025 \\
\hline Language*Time*Grade & 17.054 & 17.054 & 1 & .440 & .511 & .011 \\
\hline Error (Language*Time) & 1513.279 & 38.802 & 39 & & & \\
\hline Modality*Time & 28.759 & 28.759 & 1 & .609 & .440 & .015 \\
\hline Modality*Time*Grade & 74.997 & 74.997 & 1 & 1.589 & .215 & .039 \\
\hline Error (Modality*Time) & 1840.661 & 47.196 & 39 & & & \\
\hline Language*Modality*Time & 3.356 & 3.356 & 1 & .215 & .645 & .005 \\
\hline Language*Modality*Time*Grade & 89.810 & 89.810 & 1 & 5.765 & .021 & .129 \\
\hline Error (Language*Modality*Time) & 607.526 & 15.578 & 39 & & & \\
\hline
\end{tabular}




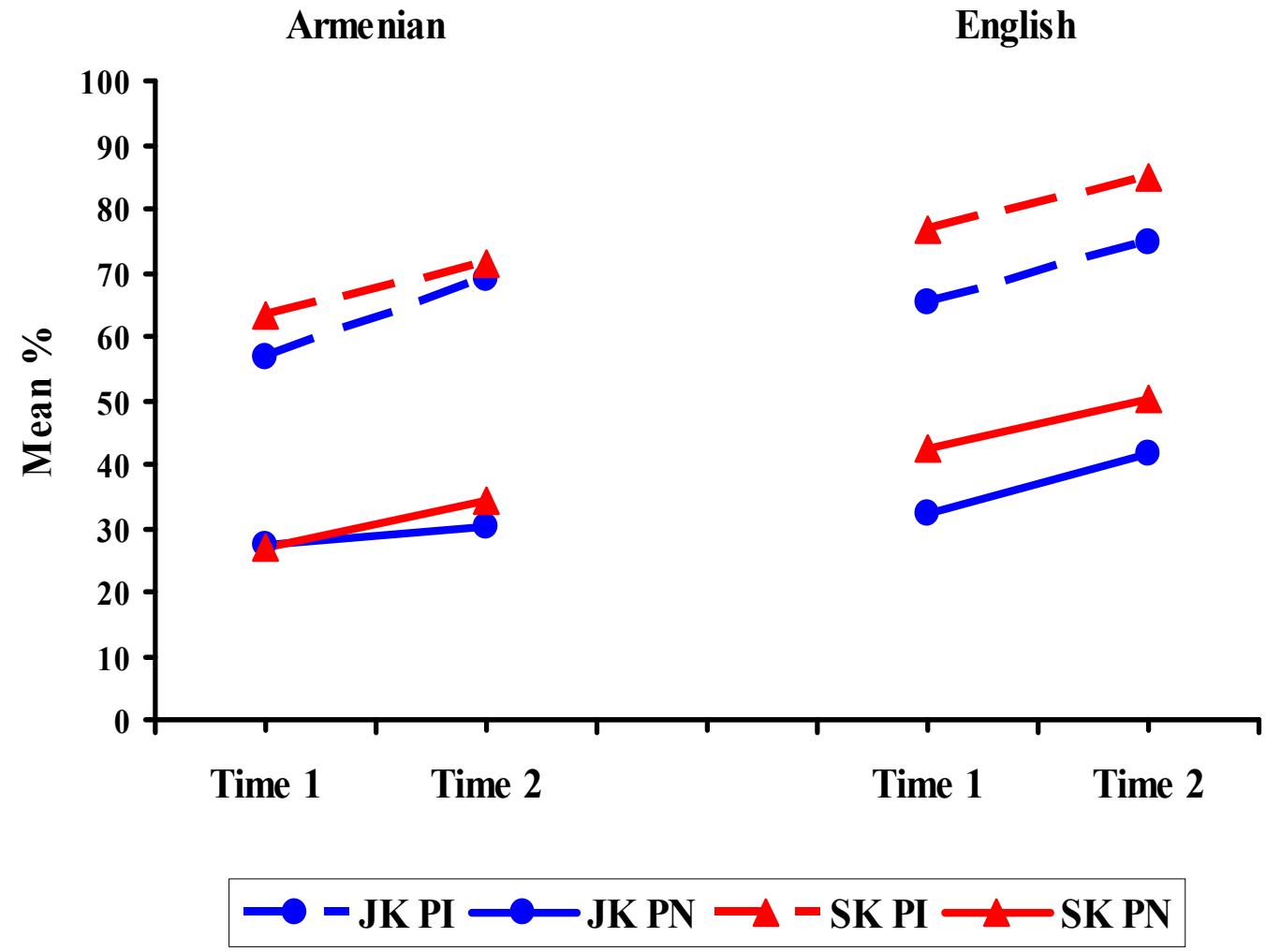

Figure 5. Significant 4-way interaction of Language by Modality by Time by Grade with lines representing adjusted mean percent correct scores in Armenian (left panel) and English (right panel) picture identification (PI) and picture naming (PN) tasks in Junior Kindergarten (JK) and Senior Kindergarten (SK) grades. 


\subsection{Relative Vocabulary Proficiency and Language Exposure}

Supplementary analyses were also conducted in an attempt to understand why two of the original predictions were not supported. These predictions were that there would be higher performance in Armenian vocabulary compared to English vocabulary and more growth in English vocabulary compared to Armenian vocabulary. Both predictions were based on the assumption that the participants might be generally representative of bilingual children with higher exposure to Armenian than English language at home, and thus with higher proficiency in Armenian vocabulary than in English vocabulary. However, these children were expected to show a higher rate of growth in English vocabulary compared to Armenian because of increased exposure to English language at school. To find a plausible explanation for lack of support for these predictions, patterns of relative proficiency in Armenian and English vocabularies were examined among the participants. The results were intended to provide information on the proportion of children who showed higher or balanced vocabulary proficiency in one language versus the other. As introduced in Chapter 1, language dominance is considered to be a relative measure of proficiency in the two languages of bilinguals. However, the focus of this supplementary analysis was only on vocabulary performance and proficiency, because the available data were not considered sufficient to provide a comprehensive picture of language dominance. To determine language dominance, several measures of language performance (e.g., vocabulary, syntax, verbal fluency) are usually taken into consideration. The supplementary analysis can however, provide a general picture of relative proficiency in Armenian and English vocabularies. In addition, given the post-hoc nature of this analysis, the results should be interpreted cautiously.

Consistent procedures were employed to examine patterns of relative vocabulary proficiency across the two languages and to identify subgroups of children with distinctive differences between vocabulary proficiency in Armenian versus English. The Armenian and English vocabulary scores at Time 1 were closely examined at the individual level. The difference between Armenian and English vocabulary performances or scores was considered as a measure of relative proficiency in the vocabularies of the two languages. An arbitrary cut-off point of 10\% difference between Time 1 Armenian and English vocabulary scores was used as a vocabulary-based criterion to identify bilingual vocabulary proficiency patterns among participants. This vocabulary-based criterion was developed on the assumption that a difference 
of at least $10 \%$ would probably be enough to suggest higher proficiency in the language with the higher score. Time 1 scores were chosen because they would show vocabulary proficiency patterns at the beginning of the study, otherwise proficiency might be influenced by the systematic language exposure children received after six months at school. A wide range of scores was observed in the vocabularies of the two languages. Some participants showed obviously higher performance in one language than the other and others showed similar performances in the two languages.

Using the vocabulary-based criterion, the participants were classified into three subgroups of vocabulary proficiency based on the difference between their Time 1 Armenian and English vocabulary scores. The first subgroup consisted of children with a balanced vocabulary proficiency $(n=16)$, participants with a maximum difference of $10 \%$ between their Time 1 Armenian and English vocabulary scores. The children in this subgroup were considered to have a balanced performance in Armenian and English vocabularies. The second subgroup consisted of Armenian-proficient bilinguals $(n=6)$, participants whose Time 1 Armenian vocabulary score was at least $10 \%$ greater than their Time 1 English vocabulary score. The third subgroup included the English-proficient bilinguals $(n=20)$, participants whose Time 1 English vocabulary score was at least $10 \%$ greater than their Time 1 Armenian vocabulary score. There was a wide discrepancy in the English and Armenian vocabulary scores in the English-proficient subgroup. A difference of more than 10\%, which corresponded to 12 items in actual performance, between Armenian and English vocabulary scores, favoring English, was considered to be an indication of higher proficiency in English vocabulary, however in most cases the difference between English and Armenian scores ranged between 20 to $50 \%$. In contrast, in the Armenian-proficient subgroup the range of discrepancy in Armenian and English vocabulary scores in most cases was less than $20 \%$. In other words, the difference between Armenian and English vocabulary scores, favoring Armenian, did not exceed $20 \%$ for most children.

The children in the JK and SK grades showed distinct differences in their patterns of relative proficiency in Armenian and English vocabularies. Based on their vocabulary performances, $25 \%$ of children in the JK were classified as Armenian-proficient compared to $4.5 \%$ in the SK. Another $25 \%$ of children in the $\mathrm{JK}$ were classified as English-proficient compared to $68.2 \%$ in 
the SK. Finally, half the children in the JK, compared to $27.3 \%$ in the SK, were classified as bilinguals with balanced proficiency in their Armenian and English vocabularies.

Parents were also questioned about the language in which they considered their children to communicate better. Previous literature has provided evidence on the accuracy of the parents in rating their children's language proficiency. In their study on young Mandarin-English bilinguals, Sheng et al. (2011) reported significant correlations between parental ratings of Mandarin proficiency and the actual vocabulary scores in Mandarin picture identification $(r=$ $.68)$ and picture naming $(r=.68)$, and between parental ratings of English proficiency and the actual vocabulary scores in English picture identification $(r=.77)$ and picture naming $(r=.74)$. Therefore, parents seem to be accurate sources of information regarding their children's language status. However, the results of the present study indicated some discrepancies between parental judgment and the vocabulary-based proficiency patterns. Two participants were missing data on the parental judgment, one who was Armenian-proficient and another who was Englishproficient according to the arbitrary vocabulary-based criterion. Overall, based on data from the remaining 40 participants, $60.0 \%$ of the judgments provided by parents were consistent with the vocabulary-based proficiency classifications described above. The parental judgment for all five Armenian-proficient children matched with the vocabulary-based proficiency of their children. However, there were inconsistencies between parental judgment and the vocabulary-based results of proficiency for 11 of 16 balanced and two of 19 English-proficient children who were judged as Armenian-proficient. In addition, parental judgments matched with vocabulary-based results for 15 of the 19 English-proficient children, but did not match for one of the 16 balanced children judged as English-proficient. Furthermore, parental judgments and vocabulary-based results were consistent for four of the 16 balanced children, but were inconsistent for two of the 19 English-proficient children who were judged as having balanced proficiency in Armenian and English.

Next, as shown in Table 9, the Armenian and English vocabulary mean scores (picture identification and picture naming combined) at Time 1 and Time 2 were calculated for each subgroup. Based on these scores, Figure 6 depicts Armenian and English vocabulary growth in the three subgroups, respectively. As illustrated in the middle panel in Figure 6, the pattern of vocabulary growth in the Armenian-proficient subgroup was consistent with the predicted Language by Time interaction. Specifically, English vocabulary showed a higher rate of growth 
than Armenian vocabulary. Care should be taken in interpreting and generalizing these results, because they were based on mean values from a small number of participants, which could not be tested statistically.

As shown in the right and left panels in Figure 6, both languages showed relatively similar patterns of growth in the balanced and English-proficient subgroups. A Language (Armenian, English) by Time (Time 1, Time 2) by Subgroup (Balanced, English-proficient) ANOVA showed no significant differences between the vocabulary growth patterns in these two subgroups, $F(1,34)=1.613, p=.213, \eta^{2}=.045$. Based on the logic underlying the hypothesis on the original Language by Time interaction, it was also expected that in the English-proficient subgroup, vocabulary growth rate would be higher in Armenian compared to English. However the small and nonsignificant difference observed between Armenian and English vocabulary growth in this subgroup did not support this expectation.

To further examine the pattern of language exposure in these three subgroups, descriptive statistics on their home language exposure are presented in Table 9. Judging from the mean values in Armenian and English, there was an unusual pattern of home language exposure in the two languages. Children in all three subgroups were exposed to more Armenian than English at home. Higher exposure to Armenian compared to English should suggest higher proficiency in Armenian than in English vocabulary, whereas only a limited number of children, i.e., the Armenian-proficient subgroup, showed this pattern. The balanced and English-proficient subgroups showed a pattern of higher vocabulary performance in English compared to Armenian despite higher home exposure to Armenian than English.

A plausible explanation for this unusual pattern is that there might be other important sources of English language exposure in addition to the exposure that children received at home through interactions with their parents and siblings. To examine this possibility answers to two additional questions on the parental questionnaire were studied. These questions provided information on the extent of language use by the child with playmates and while watching television. This information was not used in the language exposure measure because it was assumed that young children received their main language exposure at home through input from their parents and in turn used their languages most with their parents and siblings. As shown in Table 9, the data on watching television suggested that this activity provided a source of English language exposure 
that was similar for all three subgroups. However, the data on playmates showed a pattern of Armenian versus English language exposure that was consistent with the patterns of vocabulary proficiency in the three subgroups. The Armenian-proficient subgroup used Armenian most often with playmates; the English-proficient subgroup used English most often; and the balanced subgroup showed similar use of Armenian and English with playmates. One might question the accuracy of parental ratings of language use with peers and siblings. However, given the young age of children it seems logical that parents usually have the opportunity to observe children through their interactions with their siblings and peers. If the children were older, there could be more instances of peer or sibling interactions that would take place without the presence of parents. However, this question could not be addressed objectively in the present study, because of lack of data.

The last two rows in Table 9 provide descriptive statistics on the PSTM and nonverbal IQ scores in the three vocabulary proficiency subgroups. This information provides further details on the patterns of PSTM and nonverbal cognitive abilities in the three subgroups, which might be of interest to describe their vocabulary proficiency status. However, as shown, the scores are quite similar in the three subgroups. 
Table 9.

Means (M) and Standard Deviations (SD) in Three Subgroups of Vocabulary Proficiency for Percent Correct Scores in Vocabulary Tasks, Percentages of Language Exposure and Language Use, Standard Score in Nonverbal IQ, and Percent Correct Score in PSTM

\begin{tabular}{ccc}
\hline & \multicolumn{3}{c}{$\begin{array}{c}\text { Subgroups } \\
\text { Balanced }\end{array}$} & Armenian-Proficient & English-Proficient \\
\hline$(n=16)$ & $(n=6)$ & $(n=20)$ \\
$M(S D)$ & $M(S D)$ & $M(S D)$
\end{tabular}

Vocabulary Tasks

Time 1

$\begin{array}{llll}\text { Armenian } & 49.20(9.15) & 48.48(13.00) & 37.68(12.55) \\ \text { English } & 51.19(9.29) & 32.72(13.81) & 63.63(9.30)\end{array}$

Time 2

$\begin{array}{llll}\text { Armenian } & 55.45(9.45) & 55.45(10.86) & 46.81(14.70) \\ \text { English } & 59.14(13.98) & 46.81(14.18) & 71.04(10.21)\end{array}$

Language Exposure

$\begin{array}{lccc}\text { Armenian } & 86.09(15.38) & 73.94(29.27) & 58.17(30.44) \\ \text { English } & 10.20(12.79) & 6.05(10.72) & 41.22(29.35) \\ \text { Other } & 3.54(9.68) & 20.00(26.17) & 0.60(2.70)\end{array}$

Playing with Playmates

$\begin{array}{lccc}\text { Armenian } & 55.85(31.77) & 78.33(17.22) & 22.25(25.46) \\ \text { English } & 44.14(31.77) & 20.00(14.14) & 77.75(25.46) \\ \text { Other } & 0.00(0.00) & 1.66(4.08) & 0.00(0.00)\end{array}$

Watching Television

\begin{tabular}{lccc} 
Armenian & $22.50(29.27)$ & $23.33(39.32)$ & $21.75(34.03)$ \\
English & $71.25(28.13)$ & $58.33(39.70)$ & $76.75(35.06)$ \\
Other & $6.25(15.53)$ & $18.33(32.50)$ & $1.50(6.70)$ \\
verbal IQ & $115.19(16.45)$ & $116.83(14.17)$ & $114.20(12.35)$ \\
Me & $83-151$ & $102-137$ & $98-147$ \\
$\mathrm{M}$ & $39.58(12.30)$ & $37.65(21.00)$ & $41.29(11.97)$ \\
\hline
\end{tabular}


Note. Vocabulary Tasks = Mean percent correct scores in Armenian and English vocabulary tasks (picture identification and picture naming combined) at Time 1 and Time 2; Language Exposure $=$ Mean percentages of use of languages at home (Armenian, English, other) by parents with the child and by the child with parents and siblings; Playing with Playmates $=$ Percentages of use of languages (Armenian, English, other) with playmates; Watching Television = Percentages of use of languages (Armenian, English, other) in watching television; Nonverbal IQ $=$ Standard score $(M=100 ; S D=15)$ from the Brief IQ Screener, Leiter International Performance Scale-Revised; PSTM = Percent correct score in Hebrew Pseudo-Word Phonological Short-Term Memory task. 


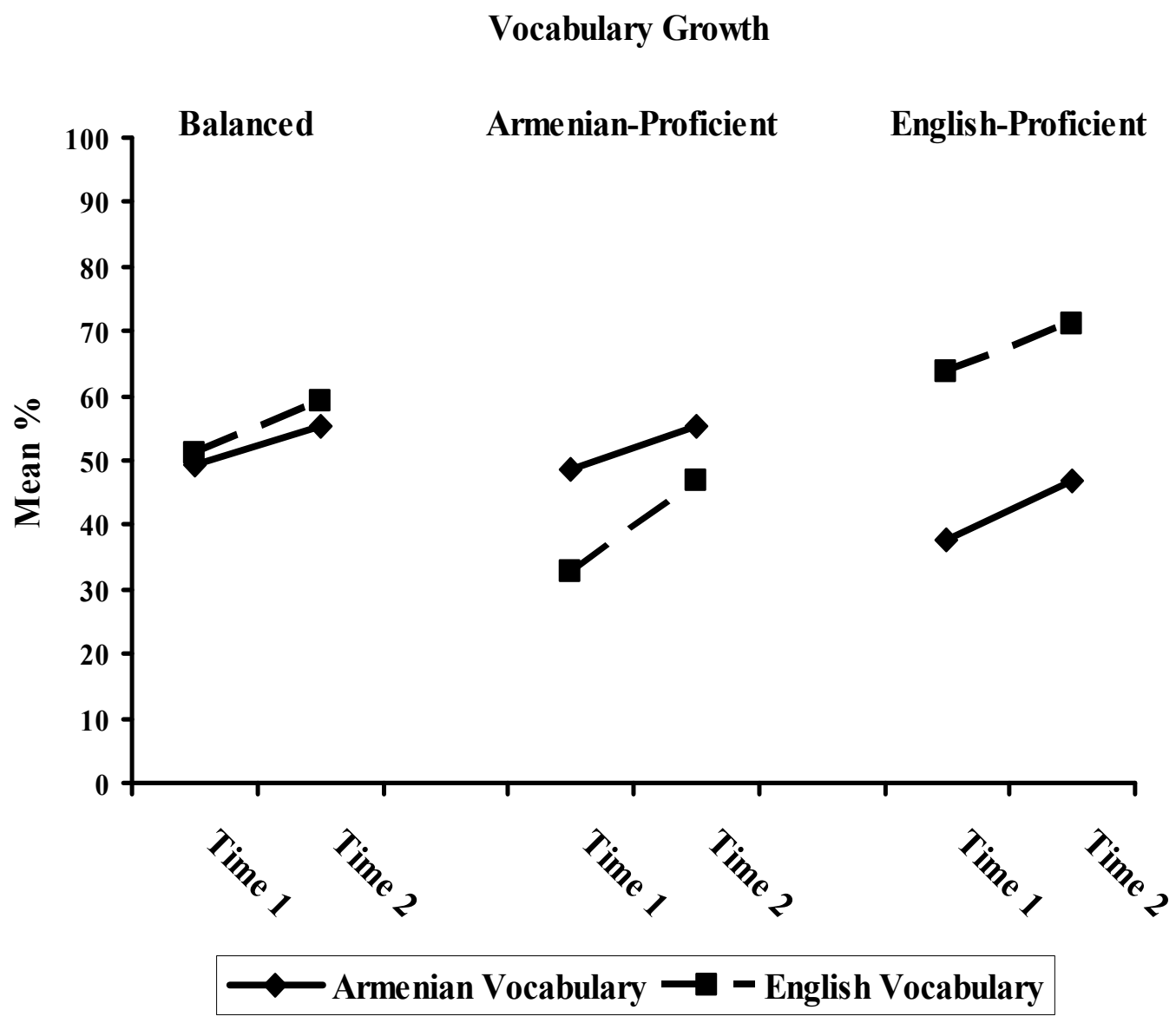

Figure 6. Vocabulary growth in the three subgroups of Balanced, Armenian-Proficient, and English-Proficient bilinguals with lines representing mean percent correct scores in Armenian and English vocabulary tasks at Time 1 and Time 2. 


\subsubsection{Individual Differences in Vocabulary Performance}

\subsubsection{Potential Predictors of Armenian and English Vocabularies}

The second objective of the study involved examining individual differences in Armenian and English vocabulary performances. To address this objective two separate categories of environmental and child characteristics were considered as potential predictors of vocabulary performance in the two languages of the bilinguals in this study. By including two categories of predictors it was possible to assess not only the contribution of each category of predictors separately but also their combined contribution to vocabulary performance in the two languages. The vocabulary scores used to represent vocabulary performances in Armenian and English were the mean of Time 1 and Time 2 percent correct vocabulary scores in each language. The mean scores were considered to be better representations of general vocabulary performance in each language, as compared to single scores at Time 1 or Time 2, because performances at both times of testing were taken into account.

To examine individual differences in Armenian and English vocabulary performances, information was obtained on five characteristics at Time 1. These characteristics, as shown in Table 4, were: (1) parental education; (2) language exposure (Armenian and English) at home; (3) language value (Armenian and English); (4) nonverbal cognitive ability (nonverbal IQ); and (5) phonological short-term memory (PSTM). Language value was not included in the analyses because ceiling effects resulted in low variability in the scores for both languages. The remaining characteristics formed the two categories of predictors in the subsequent analyses of individual differences. The first two characteristics, parental education and language exposure (Armenian and English) at home, formed the environmental predictors category, and the next two characteristics, nonverbal IQ and PSTM, formed the child predictors category.

Separate hierarchical multiple regression analyses were performed for Armenian and English. It was predicted that each category of environmental and child predictors would separately predict vocabulary performance in Armenian and English. In what follows, first the correlations among the predictors and the Armenian and English vocabulary scores are reported, and then the predictive value of these predictors are examined. 


\subsubsection{The Relationships among Environmental and Child Predictors and Armenian and English Vocabularies}

Table 10 presents the correlation matrix for the variables involved in the analysis of individual differences. Two separate panels are presented in Table 10, the top panel represents the Pearson correlation coefficients of the predictors with Armenian vocabulary and the lower panel represents the correlations with English vocabulary.

As shown in Table 10, different patterns of correlations emerged in Armenian and English. Armenian vocabulary performance correlated significantly with all four predictors in the two categories of environmental predictors, i.e., Armenian language exposure, $r(38)=.62, p<.001$, parental education, $r(38)=.29, p=.038$, and child predictors, i.e., PSTM, $r(38)=.40, p=.007$, nonverbal IQ, $r(38)=.30, p=.032$. In contrast, English vocabulary performance correlated significantly with only two predictors, the environmental predictor of English language exposure, $r(38)=.37, p=.011$ and the child predictor of PSTM, $r(38)=.39, p=.008$. As shown in Table 10, there were no significant correlations between Armenian or English language exposure and PSTM. There was a modest correlation between the two child predictors of PSTM and nonverbal IQ, $r(38)=.30, p=.035$. However, this correlation was not too strong to raise collinearity concerns in the subsequent regression analyses. 
Table 10 .

Pearson Correlations among Vocabulary and the Predictors (Armenian in top panel; English in the bottom $[N=38]$ )

\begin{tabular}{|c|c|c|c|c|c|}
\hline & 1 & 2 & 3 & 4 & 5 \\
\hline 1. Armenian Vocabulary & & $.62 * *$ & $.29 *$ & $.40 * *$ & $.30^{*}$ \\
\hline 2. Armenian Language Exposure & & $\longrightarrow$ & .06 & .11 & .18 \\
\hline 3. Parental Education & & & $\underline{-}$ & $.34 *$ & .08 \\
\hline 4. PSTM & & & & + & $.30^{*}$ \\
\hline 5. Nonverbal IQ & & & & & $\underline{-}$ \\
\hline 1. English Vocabulary & 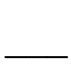 & $.37 *$ & .26 & $.39 * *$ & .23 \\
\hline 2. English Language Exposure & & $\underline{ }$ & .09 & -.06 & -.13 \\
\hline
\end{tabular}

Note. Armenian Vocabulary $=$ Mean percent correct score in Armenian vocabulary tasks (Time 1 and Time 2 picture identification and picture naming tasks combined); Armenian Language Exposure $=$ Mean percentage of use of Armenian at home by parents with the child and by the child with parents and siblings; Parental Education = Mean years of parents' education; PSTM= Percent correct score in Hebrew Pseudo-word Phonological Short Term Memory Task; Nonverbal IQ = Standard score in Brief IQ Screener; English Vocabulary $=$ Mean percent correct score in English vocabulary tasks (Time 1 and Time 2 picture identification and picture naming tasks combined); English Language Exposure = Mean percentage of use of English at home by parents with the child and by the child with parents and siblings.

$* p<.05$

$* * p<.01$ 


\subsubsection{Environmental and Child Predictors of Armenian Vocabulary}

To identify the best predictors of Armenian vocabulary performance two hierarchical multiple regression analyses were conducted. In these analyses, mean Armenian vocabulary score was the dependent variable; environmental characteristics (parental education, Armenian language exposure) and child characteristics (nonverbal IQ, PSTM) were the independent variables or potential predictors. In one of the regression analyses, the environmental predictors were first forced into the regression then the child predictors were entered in the second step. In the second analysis, child predictors were forced first into the regression and environmental predictors were entered in the second step. These regression analyses assessed three models for the contribution of environmental and child predictors to vocabulary performance. The first model or the 'environmental predictors' model provided information on the contribution of environmental characteristics only to Armenian vocabulary performance. The second model or 'child predictors' model provided information on the contribution of child characteristics only to Armenian vocabulary performance. The third full model or 'environmental and child predictors' model provided information on the additional contribution of child predictors over and above environmental predictors in the first regression and on the additional contribution of environmental predictors over and above child predictors in the second regression.

Table 11 presents the results of the hierarchical regression analyses predicting Armenian vocabulary performance. The top panel represents the first model, which assessed the contribution of the 'environmental predictors' to Armenian vocabulary. As expected, this model was significant, $F_{2,37}=14.051, p<.001$. With an adjusted $R$ square of .41 this model accounted for $41 \%$ of variance in Armenian vocabulary. As shown in Table 11, Armenian language exposure at home was the only significant variable in this model, $\beta=.602, p<.001$.

The middle panel in Table 11 represents the second model, which assessed the contribution of the 'child predictors' to Armenian vocabulary. Again, as expected, this second model was also significant, $F_{2,37}=4.251, p=.022$. With an adjusted $R$ square of .15 this model accounted for $15 \%$ of variance in Armenian vocabulary. PSTM was the only significant variable in this model, $\beta=.336, p=.042$.

The bottom panel represents the third model, which assessed the joint contribution of environmental and child predictors to Armenian vocabulary performance. This third model was 
also significant, $F_{4,37}=9.143, p<.001$. The adjusted $R$ square of .47 indicated that environmental and child predictors together accounted for $47 \%$ of variance in Armenian vocabulary. However, Armenian language exposure was the only significant variable in this full model, $\beta=.558, p<$ .001. In addition, reversing the order of entry showed that, in the full model, after the variance attributable to environmental predictors was taken into account, child predictors accounted for an additional $5 \%$ of variance in Armenian vocabulary, but this increment was not significant, $p=$ .076. Environmental predictors, however, accounted for an additional $32 \%$ of variance, $p<.001$, in Armenian vocabulary, over and above the variance attributable to child predictors.

In summary, the first two models presented in Table 11 supported the prediction of significant contribution of each category of environmental and child characteristics to Armenian vocabulary performance, separately. However, by reversing order of entry in the third model, only environmental characteristics, in particular Armenian language exposure, showed a unique contribution to Armenian vocabulary. 


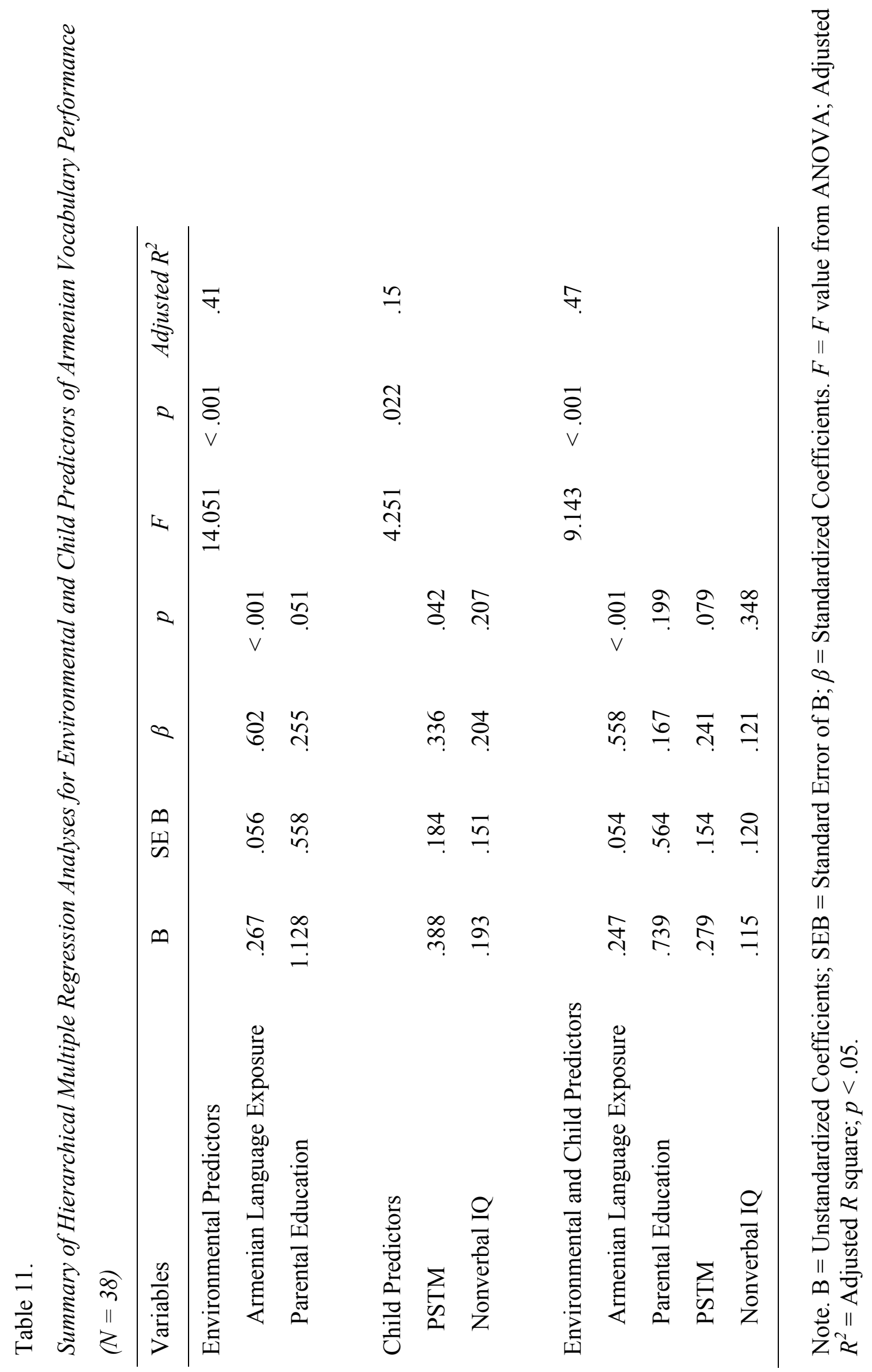




\subsubsection{Environmental and Child Predictors of English Vocabulary}

To identify the best predictors of English vocabulary performance two hierarchical multiple regression analyses were conducted. In these analyses, mean English vocabulary score was the dependent variable and English language exposure was used as a predictor instead of Armenian language exposure. The other environmental and child predictors were the same as in the Armenian vocabulary analysis, as were the regression methods.

Table 12 presents the results of the hierarchical regression analyses predicting English vocabulary performance. The top panel represents the first model, which assessed the contribution of the 'environmental predictors' to English vocabulary. As expected, this model was significant, $F_{2,37}=3.994, p=.027$. With an adjusted $R$ square of .14 this model accounted for 14\% of variance in English vocabulary. As shown in Table 12, English language exposure at home was the only significant variable in this model, $\beta=.349, p=.029$.

The middle panel in Table 12 represents the second model, which assessed the contribution of the 'child predictors' to English vocabulary. Again, as expected, this second model was also significant, $F_{2,37}=3.436, p=.043$. With an adjusted $R$ square of .12 this model accounted for $12 \%$ of variance in English vocabulary. PSTM was the only significant variable in this model, $\beta$ $=.346, p=.039$.

The bottom panel represents the third model, which assessed the joint contribution of environmental and child predictors to English vocabulary performance. This third model was also significant, $F_{4,37}=4.277, p=.007$. The adjusted $R$ square of .26 indicated that environmental and child predictors together accounted for $26 \%$ of variance in English vocabulary. English language exposure, $\beta=.405, p=.008$, and PSTM, $\beta=.324, p=.047$, were the two significant predictors in this full model. In addition, reversing the order of entry showed that, in the full model, after the variance attributable to environmental predictors was taken into account, child predictors accounted for an additional $12 \%$ of variance, $p=.030$, in English vocabulary, over and above the variance attributable to environmental predictors. Environmental predictors accounted for an additional $14 \%$ of variance, $p=.020$, in English vocabulary, over and above the variance attributable to child predictors. 


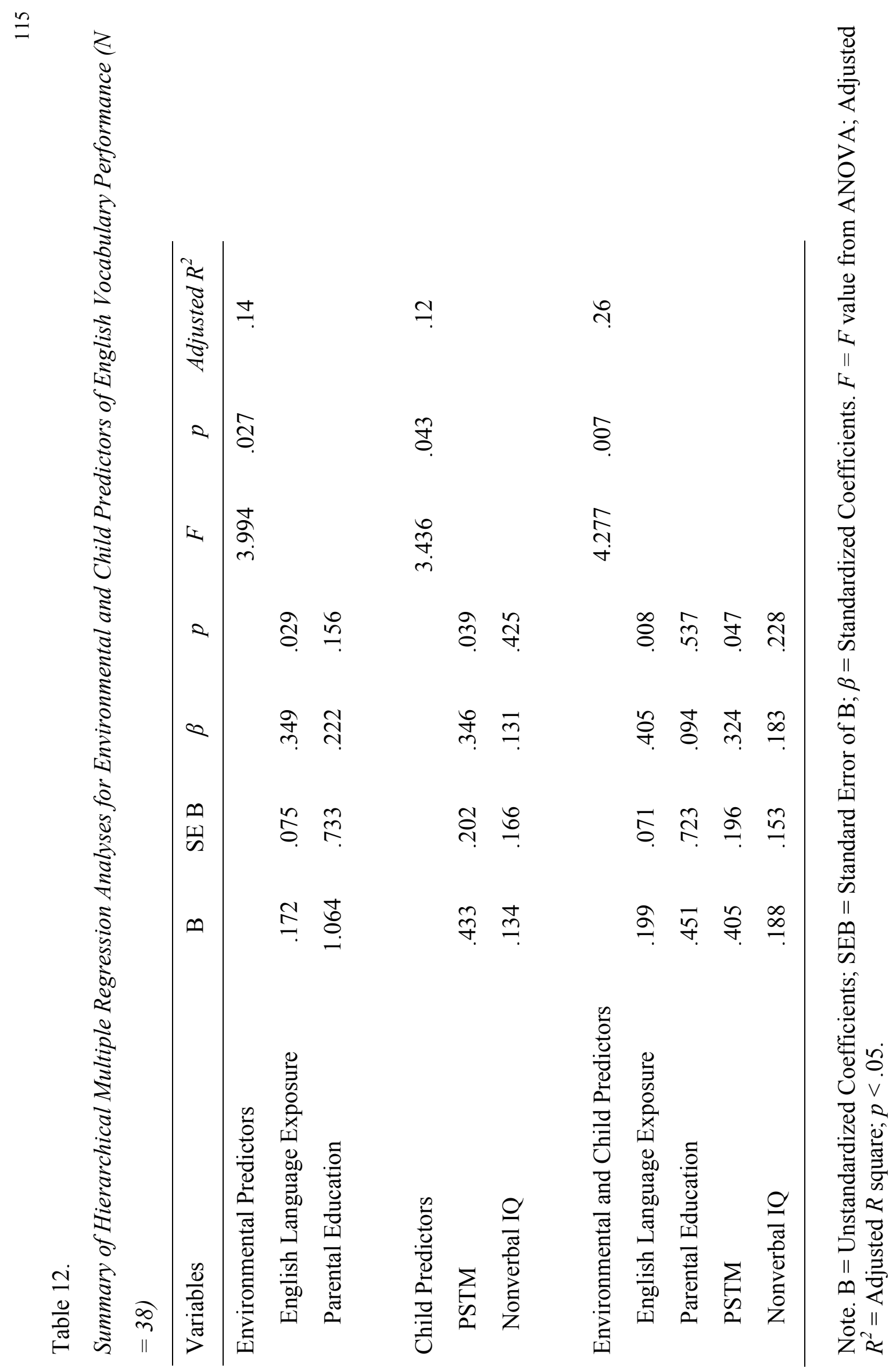


In summary, the first two models presented in Table 12 supported the prediction of significant contribution of each category of environmental and child characteristics to English vocabulary performance, separately. In addition, by reversing order of entry in the third model, both environmental characteristics, in particular English language exposure at home, and child characteristics, in particular PSTM, showed unique contributions to English vocabulary. 


\section{Discussion}

The main purpose of this study was to investigate vocabulary growth in the two languages of Armenian-English bilingual children in Junior Kindergarten (JK) and Senior Kindergarten (SK) grades. The strength of this study hinges on the validity of the parallel bilingual vocabulary test developed and administered to participants. In this chapter, the results regarding the development of the vocabulary test are discussed first, and then the results of the administration of the vocabulary test are discussed in terms of the findings on language preference, language exposure, and vocabulary proficiency. The discussion then focuses on predictors of Armenian and English vocabularies. At the end, limitations, contributions, and future directions of the study are discussed.

\subsection{The Parallel Bilingual Vocabulary Test}

The development of the parallel bilingual vocabulary test was a novel and important contribution. This study was the first to use a vocabulary measure that provided four tasks equivalent in rated age of acquisition (AoA) for measuring vocabulary performance in receptive and expressive modalities of the two languages of bilinguals with no overlap in the items used in any of the tasks. This unique feature made it possible to compare vocabulary performance across modalities and languages with confidence. Previous studies did not permit such comprehensive comparisons.

Previous researchers have tried to develop vocabulary tests that are applicable to both languages of bilinguals. However, these tests were not necessarily equivalent for receptive and expressive modalities of the two languages. For example, a bilingual Hmong-English vocabulary test was developed by Kan and Kohnert (2005). They used the same items across the two languages, however different items were used in the picture identification and picture naming tasks. This may have made the lists nonequivalent across the two modalities. As mentioned in the previous chapters, the vocabulary test developed and used in the present study consisted of four lists equivalent in rated AoA with no repeated target items. These lists were then counterbalanced across the four tasks of Armenian and English picture identification and picture naming. The equivalence of the lists on rated AoA made it possible to assess reliably the receptive and expressive modalities of both languages and to examine interactions. The cross-modality and 
cross-language comparisons provided a comprehensive overview of relative proficiency and growth in the two modalities of the two languages. However, in interpreting the results, attention should be given to the fact that the lists were not necessarily equivalent on other aspects of linguistic differences between the two languages such as morphology or word length. Therefore, this test cannot provide normative data on bilingual vocabulary growth. Nonetheless, as discussed in the following paragraphs, there is reasonable support for the equivalence of the tasks on rated AoA.

Careful procedures were followed in the development and administration of the parallel bilingual vocabulary test. These procedures complied with some of the main standards recommended for test development and administration (see American Educational Research Association; American Psychological Association; National Council on Measurement in Education, 1999). For example, initially a large pool of vocabulary items was developed so that enough suitable items could be selected for the purpose of the test. Also, in administering the test, caution was taken to test the individuals with consistent procedures in a convenient place after receiving parental consent and child assent.

The vocabulary test served the purpose of the study well, which was to capture vocabulary growth in 4- and 5-year-old Armenian-English bilingual children. The results of the administration of the test supported two of the hypotheses regarding vocabulary growth over time and differences in receptive and expressive modality performances. Based on developmental trends usually observed in monolingual as well as bilingual vocabulary, it was expected that performance at Time 2 would be higher than performance at Time 1 and performance in picture identification would be higher than performance in picture naming. The vocabulary test was sensitive to these developmental changes in both the JK and SK grades. It captured the expected vocabulary growth over time and also captured the expected gap between receptive and expressive vocabularies, i.e., picture identification was easier than picture naming.

Different measures were taken to establish the validity and reliability of the vocabulary test, as outlined in Chapters 2 and 4. First, a careful and systematic sequence of steps was laid out and followed vigilantly with the main goal of developing four equivalent and independent lists of vocabulary items. The sequence of steps developed thus covered all the stages from selecting the potential vocabulary items to developing the lists. 
In addition, correlations were indicative of the validity of the use of subjective AoA ratings for the vocabulary items included in the test. Initially, the use of subjective ratings was justified by the high correlations reported in other studies between objective measures and subjective ratings of AoA (Morrison et al., 1997). Correlations as high as .71 were also established between the subjective ratings in this study and the objective measures from published studies (e.g., Morrison et al., 1997). Finally, the adult participants in each of the three language groups, i.e., Western Armenian, Eastern Armenian, and English, who performed the AoA ratings, had high agreement in their ratings, ranging from .77 to .84 in the three groups. There was also high inter-rater agreement, with a reliability of .92 , for the three groups combined. These results suggested the validity of the procedures for obtaining the ratings.

Furthermore, there was evidence of the validity of the procedures in developing four equivalent lists both during the development of the test and after administering it. The four lists were initially developed with similar mean AoA values. In addition, the lack of an order effect in the preliminary analysis of participants' vocabulary scores indicated that all four list orders randomly assigned to the four tasks were equivalent in difficulty. A final check on the relationship between the performance of the child participants and the AoA ratings from adult participants in this study validated the procedure in ordering the items in each list from easy to difficult. The items with lower AoA values, i.e., easier ones, yielded higher vocabulary performances than the items with higher AoA values or more difficult ones.

Nonetheless, further validity studies would be valuable. For example, the concurrent validity of the vocabulary test could be assessed by including another measure of vocabulary, such as vocabulary diversity derived from spontaneous language samples in the two languages of the bilinguals (Thordardottir, 2005). Vocabulary diversity could then be compared with the results of the vocabulary test to estimate concurrent validity.

Finally, the test-reliability of the test was inferred from the results of the two administrations of the test. A strong correlation, $r=.89$, between the two times of testing indicated high test-retest reliability, suggesting that the test can be repeatedly used while providing stable results.

Overall, the vocabulary test was suitable for the research purpose of the current study. The development of the parallel bilingual vocabulary test made a unique contribution to the bilingual literature by making possible cross-language and cross-modality comparisons. Most importantly, 
the procedure used in developing this vocabulary test could be implemented for other combinations of minority-majority languages. However, this test also had some limitations, which will be discussed further in a later section.

\subsection{Language Preference}

Different rates of growth were observed in the two modalities of the minority language but not in the majority language. Specifically, children showed similar growth over six months in three of the four vocabulary tasks, i.e., Armenian picture identification, English picture identification, and English picture naming, but somewhat less growth in Armenian picture naming. This difference held across both grades. However, the two grades were different in their English language exposure at home, with the children in SK having significantly higher English language exposure compared to the children in JK. Once the two grades were statistically equated on their English language exposure, the difference in modality growth in Armenian was only observed in JK. In general, both results indicated a modality growth difference in the minority language but not in the majority language.

The overall difference between picture identification (receptive modality) and picture naming (expressive modality) performances in general is consistent with the large body of existing evidence on the difficulty of naming tasks over identification tasks. The lower performance in picture naming compared to picture identification might be related to the difficulty of recalling or naming a picture over recognizing it through picture identification. Early studies, as reviewed in Clark and Hecht (1983), have shown that understanding or recognizing a word typically precedes recalling or producing the word and that young children understand more words than they produce. It has also been shown that producing a word is harder than recognizing the word (e.g., Fromkin, 1987). Therefore, the difficulty of recall over recognition might contribute to the poorer performance in picture naming compared to picture identification. There is also evidence in the bilingual literature of higher performance of bilingual children in picture identification compared to picture naming (Kan \& Kohnert, 2005).

The overall modality difference might also be related to the different nature of picture identification and picture naming tasks measuring receptive and expressive modalities. It is generally easier to guess the answer among four pictures in a picture identification task with $25 \%$ probability of being correct, even if one is unsure about the correct answer. In addition, if one 
knows the names of three of the four pictures presented in a picture identification task trial, it is easy to guess that an unfamiliar label corresponds to the fourth (unknown) picture. In contrast, it is usually difficult to name a picture by chance without knowing the exact referent. The explanations discussed above might be applicable to overall differences in modality performance but not to the differences in modality growth observed only in Armenian in this study. However, some possible supporting evidence for a modality growth difference in the minority language was found in previous research.

For example, a modality growth difference in the minority language may have occurred in Winsler et al. (1999), whose participants, similar in age to those of the present study, were Spanish-English bilingual children attending bilingual preschools. Unfortunately, the use of different measures for the receptive modalities in each language made it difficult to compare the results on modality growth with confidence. However, as indicated by the effect sizes calculated and reported in Chapter 3, there appeared to be less growth in the Spanish (minority) expressive modality as compared to the Spanish receptive, English receptive, and English expressive modalities of the bilinguals, a pattern similar to the results of the current study.

Additional supporting evidence appears to come from the study by Rodriguez et al. (1995), whose Spanish-English bilingual participants, similar in age to the JK children in the present study, were attending bilingual preschools. Based on effect sizes calculated from their data on vocabulary growth, there appeared to be less growth in the Spanish expressive modality as compared to the Spanish receptive, English receptive, and English expressive modalities of the bilinguals. The longitudinal designs of the Rodriguez et al. and Winsler et al. studies are similar to that of the current study, however the use of different measures for the receptive modalities of the two languages is problematic in both previous studies.

Two cross-sectional studies (Kan \& Kohnert, 2005; Sheng et al., 2011) were also examined for possible evidence of modality growth differences in the minority language. For a cross-sectional study to provide supporting evidence for an expressive modality growth difference in the minority language it needed to show a similar extent of difference between two age groups in their performances in minority receptive, majority receptive, and majority expressive vocabularies, and a lesser extent of difference in minority expressive vocabulary. Although not 
as compelling as longitudinal evidence, findings from the difference in performance between two age groups could then be considered to represent growth over time.

Kan and Kohnert (2005) compared the receptive and expressive vocabulary performances of two age groups of 5- and 6-year-old Hmong-English bilingual children. As discussed in Chapter 1, the use of different measures for the two modalities made it difficult to directly compare performances across modalities, because the measures might not have been equivalent. Nonetheless, effect sizes were calculated for the difference in vocabulary performance between the two age groups in their minority and majority languages. Based on the effect sizes, there appeared to be less difference in the Hmong expressive modality $(d=.2)$ as compared to the Hmong receptive $(d=.6)$, English receptive $(d=1.7)$, and English expressive $(d=1.5)$ modalities. These results may be indicative of relatively less growth in minority expressive vocabulary, as compared to the other three modalities. However, contrary to the present study, the results may also be indicative of lower growth in both modalities of the minority language as compared to both modalities of the majority language.

In a more recent cross-sectional study, Sheng et al. (2011) compared vocabulary performances of two age groups of 3- to 5- and 6- to 8-year-old Mandarin-English bilingual children. As in the previous study, effect sizes were calculated and there appeared to be less difference in the Mandarin expressive $(d=.1)$ and Mandarin receptive $(d=.1)$ modalities as compared to the English receptive $(d=1.1)$ and English expressive $(d=1.8)$ modalities. These results suggested less growth in minority language vocabulary in general, as compared to majority language vocabulary. In addition, there was a seemingly unusual finding. The effect size in the majority expressive modality was higher than that in the majority receptive modality. This was probably the result of ceiling effects, because the older group scored above $90 \%$ in the receptive modality of the majority language.

Overall, the longitudinal studies mentioned above provided the most compelling evidence for a possible modality growth difference in the minority language. As in the current study, these studies showed less growth in the minority expressive modality but similar growth in the minority receptive, majority receptive, and majority expressive modalities. Less compelling evidence came from the cross-sectional studies. Specifically, the results of the cross-sectional 
studies also suggested relatively slow growth in the minority receptive modality, perhaps indicating that minority vocabulary in general was thriving less than the majority vocabulary.

In all four studies discussed above, the majority receptive and expressive modalities showed similar patterns of growth across time. In addition, in the present study and in the longitudinal studies where children were attending bilingual preschools, the pattern of growth in the minority receptive modality corresponded with the growth patterns in both modalities of the majority language. In contrast, in the cross-sectional studies both modalities of the minority language apparently showed less growth than the majority language. A possible explanation for this discrepancy might be the lack of formal support for the minority language at school in the crosssectional as compared to the longitudinal studies. In the Kan and Kohnert (1995) study, the minority language was only used for classroom management and the participants in the Sheng et al. (2011) study were apparently attending a monolingual English program. However, in the longitudinal studies, as well as in the present study, the schools were providing bilingual programs with support for both the minority and majority languages. Perhaps the bilingual programs in the longitudinal studies were helping children, particularly in maintaining growth in receptive minority vocabulary. However, there could be other reasons that exaggerated the difference between the growth rate in picture identification and picture naming tasks in the minority language but not in the majority language.

The modality growth difference in the minority language documented in the present as well as previous studies might reflect a preference to use the majority language. Bilingual children have been reported to show a preference to speak in the majority language from the early years in kindergarten (Oller \& Eilers, 2002). Based on observational data on Spanish-English bilinguals in school, Oller and Eilers reported that children had a tendency to speak English in the classrooms and in the hallways. They argued that this tendency might be the result of a shift toward the use of the majority language, because of longer immersion in environments with considerable exposure to the majority language and a preference to use the majority language in such environments. This might to some extent explain why children in this study showed less growth in Armenian picture naming compared to Armenian picture identification. It might be that they preferred to use the majority language more than the minority language at school, reducing the opportunity to practice the use of the minority language (Pearson et al., 1997). Language use or practice has been shown to be a significant predictor of language proficiency 
(Hakuta \& D'Andrea, 1992). Less practice in the minority language might explain the relatively slower development of ability in naming pictures in Armenian.

The preference to use the majority language has also to some extent been related to the influence of peers who speak the majority language (Luo \& Wiseman, 2000). Bilingual children have been found to prefer to use the majority language with their siblings and friends (Garcia, 1983), especially at school where there is considerable contact with peers in a context where the majority language has high value and prestige and is used for instruction (Orellana, 1994). Thus, peer-pressure, especially from those who do not speak the minority language, may have a major influence on the use of the majority language. As mentioned earlier, as part of an informal observation during the visits to the school, the researcher noticed that English was being spoken predominantly by the children in the hallways. Although this is anecdotal and there are no data to support this observation, it is possible that the discontinuation of the balanced bilingual instruction for grades after kindergarten is contributing to decreased presence and use of Armenian among children at the school. Nonetheless, future studies would be needed to investigate this possibility more carefully.

It is also possible that structural differences between the minority and majority languages might explain the different growth rates in the two modalities of the minority language compared to the majority language. A comparison of the 220 items used in the vocabulary tasks showed a difference between the length of vocabulary items in Armenian and English. Of the Armenian vocabulary items used in the four tasks, only $24.5 \%$ were one-syllable, whereas in English $47.3 \%$ of the items were one-syllable. The percentages of two-syllable and three-syllable words in Armenian were 46.8\% and 21.8\%, respectively, whereas in English the two and three-syllable items were $37.7 \%$ and $12.7 \%$, respectively. Therefore, there were considerably more short words in English than in Armenian. However, these differences could not be controlled in the present study, because of the nature of the bilingual vocabulary test. The translation equivalents of the same vocabulary items were used in Armenian and English tasks, thus there was no control over word length differences in the two languages. Overall, it might have been easier for children to use shorter words in English than their longer equivalents in Armenian. Perhaps the translation equivalent of a concept with a shorter label in one language may be accessed easier and faster than that of a longer label in the other language of a bilingual. However, this is speculative, but might be compatible with the findings in a study on the effects of word length in naming (Meyer, 
Roelofs, \& Levelt, 2003). Longer naming latencies were found for pictures with two-syllable names than for pictures with one-syllable names.

The distributed characteristics of bilingual vocabulary knowledge (Oller \& Pearson, 2002), as described in Chapter 1, might provide another plausible explanation for the modality difference observed in the minority language. It is possible that the vocabulary items tested through the vocabulary test were occurring less frequently in the home context and were more school-related (Bialystok et al., 2010). Therefore the children could not name the pictures related to those items easily. However, they might have encountered these items at school, and therefore they could identify those items in the picture identification task. This might explain the higher rate of growth in picture identification compared to picture naming in Armenian. Along the same lines, perhaps the vocabulary items that children had difficulty naming in Armenian were part of the academic vocabulary (Cummins, 1979) and were more present in the English curriculum than in the Armenian. This might, in turn, suggest a difference in the kind of vocabulary, basic vs. academic, used in Armenian and English classes. Nonetheless, all these conclusions remain speculative in the absence of data to support them. These might be topics of further investigation in future studies.

Finally, it is possible that the difference in modality growth is not a real one. This difference might be the result of sampling error, which means this result might not replicate if the study were performed a second time. This might be especially the case if the sample is not representative of the population of the study. In fact, the representativeness of the sample is questionable because of the demographic observations of high levels of parental education and above average nonverbal IQ of the children. However, to my knowledge there are not census data available to help decide whether the sample was representative of the Armenian minoritylanguage population in Canada.

To conclude, although language preference was not addressed directly in the present study, the results regarding the different growth rates in the two modalities of the minority language suggest the possible existence of majority language preference in the children studied. This might be of concern, especially considering the bilingual environment of the children. The balanced bilingual preschools providing equal support for both minority and majority languages along with the considerable minority exposure at home, suggest an additive bilingual 
environment (Lambert, 1977). One would expect comparable growth in both languages in an additive environment. However, if children in their early stages of entering the school environment are showing signs of preference towards majority language use, then more pronounced consequences might follow in the years to come with longer and more exposure to the majority language at school (Orellana, 1994; Wong Fillmore, 1991), especially past kindergarten where more instruction time is allocated to English. Nonetheless, in the absence of longitudinal data on the same children followed over several years, it is speculative to draw further conclusions. Future studies are needed to examine this possibility and to illustrate the differential patterns of minority-majority language use among young bilingual children.

\subsection{Language Exposure and Patterns of Vocabulary Proficiency}

Before discussing the findings on relative proficiency in minority-majority vocabularies in the present study a word of caution is in place. As described in Chapter 4, the findings on vocabulary proficiency were based on post-hoc analyses performed on the data, where an arbitrary cut-off point of a 10\% difference between Armenian and English vocabulary performances was considered as the criterion for classifying the children into vocabulary proficiency subgroups in this study. Caution should therefore be used in generalizing the vocabulary-based results pertaining to proficiency.

Although it was expected that the bilinguals in this study would show competence in English, the patterns of vocabulary proficiency and the discrepancy between high minority-language exposure at home and proficiency in the majority vocabulary was an unexpected finding of this study. Given the long establishment of the Armenian community in Canada, it was expected that children from this community would show some English language abilities. However, it was assumed that the majority of participants in this study would be Armenian-proficient and representative of families with dominant use of Armenian as the home language. In fact, this latter assumption was supported by parental report indicating high exposure to Armenian at home $(M=71.06 \%, S D=28.12 \%)$. What came as an unexpected finding was the pattern of vocabulary proficiency, which indicated a discrepancy between home language use and vocabulary proficiency. In contrast to high exposure to Armenian at home, most children were found to be English-proficient (47.6\%) or balanced (38.1\%), as determined by their vocabulary 
performances. It is important to note that all of these subgroups were reported to be experiencing higher exposure to Armenian as compared to English at home.

Parents were asked to judge the language in which they thought their children communicated better. Following previous literature, parents were considered to be the best available source to provide information on their children's language use and proficiency (Thordardottir \& Weismer, 1996; Gutierrez-Clellen \& Kreiter, 2003; Sheng et al., 2011). However, in the present study parental judgments did not match consistently with the vocabulary-based proficiency of their children. Parents judged many children as Armenian-proficient (11 of 16 balanced and two of 19 English-proficient), whereas based on vocabulary performance only five of the 40 children were Armenian-proficient. Although parents underrated their children's proficiency in English and overrated their proficiency in Armenian, their judgments were consistent with the vocabularybased proficiency for 15 of the 19 English-proficient and all five Armenian-proficient children. The discrepancy between parental judgments and test results regarding proficiency patterns might be explained by the fact that the majority of parents (over $84 \%$ ) were first-language speakers of Armenian and their main interactions with their children were in Armenian. Therefore, parents could not always get a consistent idea about their children's English proficiency, because English did not appear to be used very often at home. In addition, the method used in this study was different from that employed in Sheng et al.'s (2011) study for obtaining parental ratings of child language proficiency. The ratings in the present study were based on only one question regarding the language in which parents thought that their children communicated better, whereas in Sheng et al.'s the parents were asked to rate their child's proficiency in Mandarin and English in vocabulary, grammar, sentence length, pronunciation, and listening comprehension, separately, using a five-point rating scale. Then an average score was obtained for each child for each language, which represented the overall proficiency in that language. This methodological difference might explain, to some extent, the discrepancy between the findings related to parental ratings in the present study and in Sheng et al's.

The patterns of vocabulary proficiency showed distinct differences in the children in the JK and SK grades. Although all three subgroups were represented in both JK and SK, the proportions of children representing the three subgroups were different in the two grades. For example, one out of four children in JK was Armenian-proficient, whereas this proportion was one out of 20 in SK. As another example, the majority of the JK children were classified as having balanced 
proficiency in Armenian and English vocabularies, whereas the majority of the SK children were English-proficient. This discrepancy between JK and SK might also be related to the differences observed between the two groups in terms of English language exposure. As already mentioned, the SK children had higher exposure in English language at home, even though both groups had higher exposure in Armenian than English at home. Overall, the discrepancy in proficiency patterns emphasizes the great variability among bilinguals who belong to the same minoritylanguage community. In addition, based on the range of differences between Armenian and English vocabulary scores, it seemed that English-proficient children were experiencing a true proficiency in English, whereas the Armenian-proficient children seemed to be closer to being balanced because they showed more similar performances in Armenian and English vocabularies.

The variability among bilingual children in this study has important implications for teachers. The bilingual program that the children were attending was focused on teaching literacy in both languages. Vocabulary has an important role in literacy development (Hammer et al, 2007). Given the various levels of vocabulary skill in each language in the children, teachers face a great challenge in adapting instructions for these young learners. Armenian teachers seem to face a more challenging task of improvising for varied skills of the young learners in Armenian vocabulary, especially with the SK children who were more proficient in English vocabulary. Oral language vocabulary activities might be one of the ways to enrich both oral and written skills. In addition, the possible existence of language preference favouring the use of English among the children should be considered crucial, because it might have adverse consequences on the use of the minority language over time. If the observed possible trend of majority-language preference continues, children would gradually substitute the majority language for the minority language in different contexts, decreasing the chance to use and sustain proficiency in the minority language. Therefore, Armenian teachers might play an important role in maintaining Armenian use by providing more opportunities to use Armenian.

The patterns of vocabulary proficiency in the children were suggestive of high exposure to English. Based on parental report, children had considerable exposure to English $(M=57.32 \%$, $S D=34.18 \%$ ) through activities other than interaction with parents and siblings. These activities were TV watching and interactions with peers. It should be noted that these items were not included in the measure of home language exposure. Children reportedly had considerable 
exposure to the minority language at home. However, consistent with their exposure to the majority language through the activities mentioned above, specifically peer interactions, many showed proficiency in English, as measured by the vocabulary performances of the children in each of their languages. This finding suggests that exposure to the majority language through peers and perhaps other sources may have a substantial influence on children's proficiency in that language.

Siblings might also play a significant role in providing English language exposure at home. Siblings' roles have been investigated in the language environment of families. Using an ethnographic approach, Obied (2009) showed that elder siblings used both minority and majority languages at home, thus influencing the use of languages by their parents and younger siblings. In fact, the data on the percentage of use of English by children at home suggested that they were more likely to use English with their siblings (45.6\%) than with their parents (28.0\%). This might suggest an important source of English language exposure at home. However, the present study did not provide information on the use of languages by the siblings with the child, a factor that should be examined in more detail in future studies.

In a post-hoc analysis based on descriptive statistics, vocabulary growth in Armenian and English was analyzed in the three subgroups representing relative proficiency in Armenian and English vocabularies. The analyses provided some support, albeit weak, for one of the hypotheses of this study. According to this hypothesis and based on the assumption that the majority of children would have predominant use of Armenian at home, it was predicted that children would show a higher rate of growth in English compared to Armenian. In fact, the vocabulary growth of the Armenian-proficient subgroup appeared to be in line with this hypothesis, with English showing a higher rate of growth than Armenian. However, this difference in growth rate was not statistically tested because it was based on few participants and therefore cannot be generalized.

Another unexpected finding was the group difference in English language exposure at home, in which the children in SK showed higher exposure than the children in JK. This difference might be simply a matter of the difference between the two samples, which might not be replicated if the study were performed a second time. However, this difference might also be a real one, which raises the question of why the SK children should show such a difference from the JK 
children. Some demographic information might provide a plausible explanation for this difference. As mentioned in Chapter 3, the JK group included more recent immigrant families, with $58.8 \%$ of the JK families (both father and mother) as compared to $30.0 \%$ of the SK families being newer immigrants. It is more likely that families with longer establishment in the majority community experience more contact with the majority culture and more interaction with Englishspeakers, which could in turn provide significant sources of English language exposure for their children.

Once the difference in English language exposure at home between the JK and SK grades was taken into consideration through a post-hoc analysis, children in the two grades showed statistically similar performances in English vocabulary. This finding makes sense when considering the positive relationship between language exposure and vocabulary performance documented in the literature. For instance, Parra et al. (2011), who studied 2-year-old SpanishEnglish bilingual children, found a positive correlation $(r=.72)$ between the percentage of English language exposure at home, measured by a questionnaire, and expressive English vocabulary, measured by a standardized parental report inventory.

There was a change in the overall group differences in vocabulary after taking into account the difference in English language exposure at home between the JK and SK grades. Originally it was predicted that the SK children would show higher performance in vocabulary than the JK children. This predicted difference between the two grades was not observed in the initial analysis, but was observed once the difference between the grades in English language exposure was taken into account in a post-hoc analysis. In addition, the original analysis also showed higher performance in English in the SK children as compared to the JK children, but similar performances in Armenian. However, after accounting for English language exposure, this pattern was no longer significant.

English language exposure at home also accounted for the difference initially observed between the two languages. Based on the assumption of predominant use of Armenian at home, a higher performance was predicted in Armenian compared to English. However, the results of the main analyses contradicted this prediction in that performance in English was shown to be higher than performance in Armenian. Once the difference in English language exposure at home was taken into account in a post-hoc analysis, this difference was no longer observed. A similar 
performance was observed in the two languages, which again did not support the original prediction of higher proficiency in Armenian.

Although the present study did not investigate home language maintenance, the findings on English vocabulary proficiency are in line with previous studies (e.g., Duursma, RomeroContreras, Szuber, Proctor, Snow, August, et al., 2007), which suggest that children in an English-dominant culture become proficient in English despite substantial exposure to a minority language at home. In other words, there is little need to support English at home, because the majority community typically provides the necessary support. However, the Armenian language needs the support of the home environment, which plays a crucial role in the maintenance of the minority language (Hakuta \& D'Andrea, 1992). The findings regarding lower growth in Armenian expressive vocabulary as compared to receptive vocabulary may therefore be a cause for concern. Families must concentrate on the establishment of their minority language if they want their children to become proficient in both languages and to keep the balance between Armenian and English proficiency, perhaps especially in expressive vocabulary.

In addition, the use and instruction of the minority language at school also provides an environment that enhances the growth of the minority language along with the majority language. The findings by Barnett et al. (2007) suggest that a balanced bilingual program may play a role in the maintenance of the minority language. However, the current results regarding the modality difference in minority language growth as well as the findings on the high rate of English vocabulary proficiency among the children may provide some hints that the balanced bilingual program in itself is not enough to ensure balanced growth in both minority and majority languages. These findings along with previous literature (Hakuta \& D'Andrea, 1992; Luo \& Wiseman, 2000) further emphasize the importance of the home environment in the maintenance of the minority language. This emphasis becomes even more essential in the years past kindergarten because these children will be experiencing an unbalanced bilingual program with more extensive exposure to English compared to Armenian at school.

Overall, the findings related to group differences discussed above and the findings on individual differences to be discussed in the following section share some similarities. Both groups of findings are indicative of the presence and influence of language exposure as a significant factor in vocabulary performance of bilingual children. 


\subsection{Predictors of Individual Differences in Armenian and English Vocabulary Performances}

The sparse literature on individual differences in vocabulary development in minority-language bilingual children has addressed very limited age ranges and few factors. These studies have usually been conducted on very young children, and the main focus of these studies has been on language exposure (see e.g., David \& Wei, 2008; Pearson et al., 1997). The differences between the language communities of children with different minority languages may introduce some challenges in the applicability of findings regarding individual differences from a specific minority-language community to other communities. These challenges are mainly due to the existence of a wide range of variability among and between children from different minoritylanguage communities, which, in turn, is related to the different circumstances surrounding the acquisition and use of these languages. These issues call for more comprehensive studies to examine individual differences in bilingual children from minority-language communities.

The use of two different groups of factors as potential predictors of Armenian and English vocabulary performance made a unique contribution to the literature on individual differences in bilingual vocabulary. Previous studies on bilingual vocabulary have usually used one or at the most, two of these factors (e.g., Parra et al., 2011) as predictors in the analyses of individual differences in bilingual vocabulary. Therefore, in the present study, by using different factors in the analyses of individual differences in Armenian and English vocabularies it was possible to examine the contribution of these factors individually and in relation to each other. To reiterate, these factors formed two categories of potential predictors, the environmental (parental education, language exposure) and child predictors (nonverbal IQ, PSTM).

The correlations between vocabulary and the four predictors showed different patterns for Armenian and English. Consistent with previous literature, the present study showed significant correlations between Armenian vocabulary and the four predictors, namely parental education (Fenson et al., 1994; Golberg et al., 2008), language exposure (Pearson et al., 1997; Parra et al., 2011), nonverbal IQ (Leseman, 2000), and PSTM (Adams et al., 1999; Farnia \& Geva, 2011), whereas English vocabulary correlated significantly with only two of the predictors, one from each category, namely English language exposure and PSTM. The correlation of language 
exposure and vocabulary was larger in Armenian than in English, whereas the correlation of PSTM and vocabulary was similar in magnitude for the two languages.

The lack of significant correlations between Armenian or English language exposure and PSTM was in line with the initial theoretical presumption underlying the choice of the Hebrew pseudoword PSTM task. It was assumed that this measure would be independent of Armenian and English language experience, and, in fact, the lack of correlation between PSTM and Armenian and English language exposure supported this assumption. From a theoretical perspective, the choice of this test was meant to minimize the possibility of familiarity with task items, therefore rendering the test to be a purer measure of PSTM. This would, in turn, put all the participants in equivalent conditions regardless of their proficiency in their respective languages. Although previous literature showed a positive relationship between language exposure in a specific language to phonological memory in that language (Parra et al., 2011), in the present study phonological memory was essentially uncorrelated with language exposure. This gave the potential for language exposure and phonological memory to contribute separately to Armenian and English vocabulary. However, more solid conclusions could be drawn if there were specific data on the similarities and differences between Hebrew and the two languages of Armenian and English.

As expected, both environmental and child predictors predicted Armenian and English vocabulary performances when each category of predictors was considered separately. Environmental predictors alone accounted for more variance in Armenian vocabulary (41\%) than in English vocabulary (14\%). Child predictors alone accounted for similar amounts of variance in both Armenian (15\%) and English (12\%) vocabulary. For both languages, language exposure and PSTM were the only significant predictors from each category of environmental and child predictors, respectively.

However, when both environmental and child predictors were considered jointly, only the environmental predictor of Armenian language exposure was found to be a unique predictor of Armenian vocabulary. PSTM was the second best predictor of Armenian vocabulary but it did not make a unique contribution. In contrast, both the environmental predictor of English language exposure and the child predictor of PSTM, made unique contributions to the prediction of English vocabulary. Parra et al. (2011) also showed that language exposure was a unique 
predictor of both minority and majority expressive vocabulary of Spanish-English bilinguals over and above language-specific PSTM measures. However, they did not report regressions in which PSTM measures were forced to enter following language exposure so it is unclear whether both variables made unique contributions to prediction of performance in both languages.

There are two plausible explanations for the discrepancy in findings on the unique predictors of Armenian and English vocabulary performances. One plausible explanation might be related to some methodological limitations of the language exposure measure. Language exposure was more strongly associated with minority language performance $(r=.62)$ than with majority language performance $(r=.37)$. This might indicate that the language exposure measure was more robust for the minority language than for the majority language. As discussed in Chapter 3, the language exposure measure was developed primarily based on the percentages of use of languages at home by parents and siblings, while exposure from sources outside the home, such as playmates, was not included. However, the results suggested that many children had substantial exposure to English outside the home through their playmates. Therefore, the exclusion of the exposure received outside the home, which was probably more substantial in English, might render the English language exposure measure less accurate than the Armenian language exposure measure. Thus, it is possible that the variance explained by Armenian language exposure might be more reliable than the variance explained by English language exposure.

Future studies may provide a more balanced picture of the relative role of the environmental and child characteristics by using a language exposure measure that can capture exposure to both languages in a variety of contexts and environments. The use of such an exposure measure may result in similar unique predictors for both languages. Although ideal in theory, development of a comprehensive measure might be difficult in practice. Obtaining information on language exposure in different contexts and environments suggests the involvement of different individuals in child's life who interact with the child in the minority or majority languages or observation of the child in different contexts using the two languages. The development of such a measure requires very extensive questioning and/or observation, which was not considered feasible for the purpose of the present study. 
A second plausible explanation for the discrepancy in findings on the unique predictors of Armenian and English vocabulary performances might be related to the differential role of PSTM in learning new words as compared to remembering familiar words. In their review, Baddeley et al. (1998) proposed that the primary function of PSTM is to store sound patterns for unfamiliar words. They added that retaining the sound sequences of familiar words is a secondary function of PSTM. Based on this idea a differential role can be envisaged for PSTM in learning vocabulary in the children for whom Armenian might be more familiar than English. Although the majority of children were more proficient in English vocabulary than Armenian, this does not necessarily eliminate the possibility that Armenian was initially a more familiar language to these children than English. The majority of children in the present study seemed to be first-language speakers of Armenian. In fact, there is some demographic information in support of this assumption. As mentioned in Chapter 3, the majority of parents, over $83 \%$ of fathers and mothers, were first-language speakers of Armenian and their children received considerable exposure to Armenian at home through their parents. Therefore it is logical to conclude that the majority of the children were first-language speakers of Armenian for whom Armenian was initially a more familiar language with familiar sound patterns compared to English. Therefore, based on Baddeley et al.'s (1998) proposal, learning new vocabulary in Armenian was not relying so much on PSTM as it was relying on Armenian language exposure, whereas in learning English, a relatively unfamiliar language, children may have relied more on PSTM to store and learn new sound patterns.

To conclude, the findings of this study on the predictors of individual differences in minority and majority vocabularies replicated some of the findings in previous literature and provided some novel contributions. As supported by previous studies, language exposure and PSTM each contributed significantly to Armenian and English vocabularies. However, the findings regarding the unique predictors of Armenian and English vocabularies were to some extent novel contributions, because, as already stated, previous literature, with the exception of e.g., Parra et al. (2011), usually did not include different predictors in the analyses of individual differences in minority and majority vocabularies. Nonetheless, a word of caution is required regarding the interpretation of the findings of the present study on individual differences. To do multiple regressions it is recommended that there should be at least 10 participants for each predictor variable included in the analysis (Brace, Kemp, \& Snelgar, 2009). However, the number of 
participants included in these regressions was only 38 and there were four variables included in the regression analyses.

\subsection{General Limitations of the Study}

One of the limitations of this study pertains to the overall representativeness of the sample. It appears that the participants were from families with high SES. The participants in this study had high nonverbal IQ scores and high levels of parental education. In addition, families were sending their children to private schools. These known correlates of high SES are potential indications of high SES for this sample (Cobo-Lewis et al., 2002). Therefore, these features might render the participants to be a select sample, nonrepresentative of the ordinary population. However, in the absence of population norms on these characteristics from the ArmenianEnglish bilingual community, this limitation is more of a speculative than of a definitive nature.

The vocabulary test developed in the first study and used in the main study had some limitations in addition to its multiple points of strength. First, only picturable vocabulary items were used, restricting the range of potential vocabulary items. Given the nature of picturable vocabulary items, the test represents concrete items, which are generally easier than abstract ones (De Groot $\&$ Keijzer, 2000). Second, for the same reason as mentioned above, the use of the vocabulary test might be limited to younger children. Ten children scored over $90 \%$ on the English picture identification task at second time of testing, suggesting possible ceiling effects. Therefore, this test might not be as appropriate for older children. Third, the vocabulary test was not controlled for semantic and phonological features of its constituent items. These features might act as sources of variability in task performance between and within the two languages. Fourth, the analyses employed to investigate vocabulary task performance only assessed reliability of effects across differences among participants but not vocabulary items. To address this limitation, random-effects analyses could be used, which would determine whether the hypotheses were supported in spite of multiple differences across both participants and items (Baayen, Davidson, \& Bates, 2008). Nonetheless, it has been shown that when item variability is experimentally controlled by counterbalancing, as was the case in the present study, it is reasonable to use the simple subject analysis, i.e., averaging the data for each participant over items within conditions and using the means (Raaijmakers, Schrijnemakers, \& Gremmen, 1999). Finally, the way that this test was used made it impossible to examine conceptual vocabulary. Conceptual vocabulary 
provides a way to estimate the overall vocabulary knowledge of bilingual children by counting the number of words known in each language and then excluding the translation equivalents (Pearson et al., 1993). Pearson and colleagues showed comparable levels of conceptual vocabulary in bilingual and monolingual children. Therefore, measuring conceptual vocabulary in bilinguals presumably provides a realistic measure of their overall vocabulary size. To make the current test usable for measuring conceptual vocabulary, the same lists could be used in both languages, instead of counterbalancing the lists across languages. However, it should be noted that repeated use of the same lists might cause a practice effect. Nonetheless, the vocabulary test used in this study provided a reliable measure for addressing the purpose of the study, which was investigating cross-language and cross-modality comparisons as well as growth over time in vocabulary.

The language exposure measure served the purpose of this study well by capturing the amount of language exposure at home. It provided a relative measure of the percentages of use of each language at home by different individuals. However, the percentages did not provide information about the variability and nature of the use of languages by the individuals. There could be some systematic differences in the amount of talk addressed to children by different individuals. For example, even though parents might have reported equal percentages of use of language, this did not necessarily mean that both parents spent equal amounts of time interacting with the child. One parent could have been away during the day and his/her most frequent linguistic interactions might have been restricted to weekends. In addition, the context and quality of the languages used by parents might be different, again making it difficult to compare percentages.

Theoretically, the language exposure measure could be improved in three different ways. First, the measure could include additional information on different activities that provide language input to evaluate the role of these activities on vocabulary growth. For example, previous studies have shown that home literacy activities such as shared book reading nurture language development. Young bilingual children who were being read to in each of their two languages showed larger expressive vocabularies than children who never or rarely experienced this activity (Patterson, 2002). Second, the measure could also be improved by capturing variability among language users. Some studies have captured the variability by obtaining the total number of hours of exposure to each language by each individual (Marchman, Martinez-Sussmann, \& Dale, 2004; Gutiérrez-Clellen \& Kreiter, 2003). To do so, information was obtained from 
individuals in the child's daily life on the time spent with the child per week and the languages spoken to and by the child. Finally, to improve the language exposure measure more information could be obtained on language exposure outside the home, especially exposure received through peers. This information could be provided by parents or by individuals who interact with the child outside the home, such as teachers. However, in the present study teachers were not considered as an appropriate source of information on relative use of the two languages, because Armenian and English were taught by separate teachers and children in each class would use the language of instruction, either Armenian or English. This setting would not allow teachers to observe much variability in language use.

Although the inclusion of the information discussed above might help in developing a more comprehensive measure of language exposure, questions of priority and feasibility were also critical. Development and use of such an extensive measure of language exposure was deemed to be of lesser priority in the current study where the main focus was on measuring vocabulary growth of children as accurately and as reliably as possible.

\subsection{Contributions and Future Directions}

The development of the parallel bilingual vocabulary test was a novel and important contribution of this study. This test provided a valid and reliable measure for cross-language and crossmodality comparisons of bilingual vocabulary. To my knowledge this is the first study to make such comprehensive comparisons in bilingual vocabulary. In addition, the procedures used in developing the parallel vocabulary test, which was equivalent across the two languages in terms of rated AoA of the items, could be duplicated in developing bilingual vocabulary tests in any combination of minority-majority languages. This could make it possible to study vocabulary development in different groups of bilinguals from minority languages that lack standardized vocabulary measures. It is noteworthy that this test should only be used for research purposes, because it is not a standardized test.

The present study made a significant contribution to the relatively small body of literature on minority-majority bilingual vocabulary acquisition in young children in several ways. First, this study was one of the few longitudinal examinations of vocabulary growth in bilingual children. By using both a longitudinal (within grade) and cross-sectional (across grades) design, vocabulary growth was described during the first two years of schooling. 
Second, the Armenian minority-language community involved in this study offered a unique balanced bilingual program in their schools. Given the positive effects of this kind of bilingual program on minority and majority language development, as compared with an English-only program (Barnett et al., 2007), the Armenian schools provided a unique opportunity to investigate vocabulary growth in an additive environment where equal educational support was provided for both minority and majority languages.

Third, this study was the first of its kind to examine young bilingual children with Armenian as their minority language and English as their majority language. Unlike Spanish, which is widely studied in the bilingual literature and shares similarities with English, the Armenian-English combination has not been previously investigated. Therefore, the results were intended to provide new insight into bilingual vocabulary research by focusing on a language that shares almost no similarities with English. However, the findings of the present study did not seem to be largely affected by the differences between the two languages except perhaps for the difference observed between the rate of growth in Armenian picture naming compared to the rate of growth in the other three tasks. This finding, as discussed earlier, might have reflected the higher proportion of long words in Armenian compared to English. It is possible that differences between Armenian and English languages might be more readily discernible in studies on other domains of language, such as syntax, which might be a topic for future studies.

Fourth, the study also showed the influence of majority language exposure on vocabulary performance and proficiency patterns, which, as discussed, might have important implications for minority-language maintenance. Finally, by capturing the variability among participants in terms of environmental and child characteristics, the predictive value of these characteristics was investigated. This provided information on predictors of Armenian and English vocabulary performance. Given the importance of vocabulary in language (Conboy \& Thal, 2006) and literacy development (Hammer et al., 2007), this information can inform us on the features that are related to better vocabulary performance in young bilingual children.

Future studies are needed to deepen our understanding of vocabulary growth in bilingual children. From the findings of this study several directions emerge for future studies. First, longitudinal studies should be designed to include a wider age range, which can help in further identifying developmental patterns of vocabulary growth. This will provide a more 
comprehensive picture of bilingual vocabulary development through which, eventually, the ranges of typical development can be distinguished from developmental delays or deviations.

Second, it would also be interesting to follow up the participants of this study to examine their vocabulary development and language preference changes across time. This would provide more accurate information on language maintenance and the changing pattern of language use among bilinguals. Such studies can also enhance our knowledge of the factors related to language proficiency and maintenance in bilingual children, which can assist in identifying practices that may nurture the maintenance of a minority language, while learning a majority language.

Third, future studies might provide a more comprehensive picture of bilingual environments by including a measure of language exposure, which can capture language exposure not only at home but also outside the home and at school. Given the significant role of language exposure in the findings of this study, in future studies, it would be wise to use a measure that might capture additional details pertaining to language use at home and at school by different individuals, as described in the previous section. By investigating language exposure in more detail, the patterns of language use among peers and their effect on vocabulary performance can be better understood. In addition, detailed investigation of language exposure can provide information on language use among siblings, especially the influence of older siblings on language use.

Fourth, studies on the use of languages in minority and majority language classes in the bilingual program may be informative. These studies may focus on the kinds of vocabulary items used in each of the classes to see whether there are substantial differences in the nature of the vocabulary, conversational vs. academic, used in the two classes. Finally, studies are needed to investigate the relationship between vocabulary development and other aspects of language. These studies can inform theories of language development in general and theories of bilingual acquisition in particular.

\subsection{Conclusion}

This study provided information on the developmental trends in vocabulary in young bilinguals from an Armenian minority-language community who were also learning English. The findings indicated that bilingual children attending the Armenian bilingual schools were showing growth in both their minority and majority languages. However, the finding of slower rate of growth in 
Armenian expressive vocabulary compared to Armenian receptive vocabulary is a cause for concern. These findings may suggest a preference to use the majority language among the children, which might have negative consequences for minority-language maintenance in the long term. The families should be made aware of the possibility of a change in their children's language preference showing a shift towards the English language through extended exposure to English, especially at school. Minority languages, Armenian in this case, are reflections of thousands of years of cultural heritage handed down from generation to generation. It is incumbent upon new generations to keep these languages alive by having a better understanding of the dynamics of minority-language use and maintenance in a dominant English culture.

Bilingual children from minority-language families need to learn both languages so that they can connect to the family and establish a positive ethnic cultural identity, which is valued by the family. Based on the information provided in the questionnaire, the parents in this study valued the context for learning both languages by sending their children to bilingual schools. Although the bilingual program was providing a good context for learning both languages, parents should be aware of the extensive influence of English as a majority language. Children were exposed to a wide range of English in the environment through interactions with peers, to the extent that even children who received low English language exposure at home became proficient in English vocabulary. Even though the children in this study were reportedly getting high exposure to Armenian at home, exposure to the majority language was so influential that they showed relatively better performances in the majority language. Therefore, parents as well as teachers should be aware of the need to provide Armenian language exposure including literacy at home and in the school to the extent possible, so that continued Armenian use and growth will be ensured in their young children. They need to help children get the best of Armenian language exposure at home and at school, because their language practices at home along with the provision of bilingual programs at school can play a decisive role in the development, use, and maintenance of their home language (Hakuta \& D'Andrea, 1992; Luo \& Wiseman, 2000; Barnett et al., 2007). 


\section{References}

Adams, A. M., Bourke, L., \& Willis, C. (1999). Working memory and spoken language comprehension in young children. International Journal of Psychology, 34(6), 364-373.

Adams, A. M., \& Gathercole, S. E. (1996). Phonological working memory and spoken language development in young children. Quarterly Journal of Experimental Psychology Section A: Human Experimental Psychology, 49(1), 216-233.

Adams, A. M., \& Gathercole, S. E. (2000). Limitations in working memory: Implications for language development. International Journal of Language and Communication Disorders, 35(1), 95-116.

Aitchinson, J. (1994). Words in the mind: An introduction to the mental lexicon (2nd ed.). Oxford, UK: Blackwell.

Altman, G. T. M. (1995). Cognitive models of speech processing: An introduction. In G. T. M. Altman (Ed.), Cognitive models of speech processing [electronic resource]: Psycholinguistic and computational perspectives (pp. 1-23). Cambridge, MA: MIT Press.

American Educational Research Association; American Psychological Association; National Council on Measurement in Education. (1999). Standards for educational and psychological testing. Washington, DC: American Educational Research Association.

Andonian, H. (1966). Modern Armenian. New York, NY: Armenian General Benevolent Union of America.

Armenian General Benevolent Union Toronto. (2006). Retrieved October 20, 2006, from http://www.agbutoronto.ca/index.php?option=comcontent\&task=view\&id=24\&Itemid=6 $\underline{3}$

Asmangulian, H. A. \& Hovhannisian, M. I. (Eds.). (1984). English-Armenian dictionary. Yerevan, Armenia: Hayastan.

August, D., Carlo, M., Dressler, C., \& Snow, C. (2005). The critical role of vocabulary development for English language learners. Learning Disabilities Research \& Practice, 20(1), 50-57.

Awde, N. \& Maghdashyan, P. (2003). Eastern Armenian: Armenian-English, English-Armenian dictionary \& phrasebook. New York, NY: Hippocrene Books.

Baayen, R. H., Davidson, D. J., \& Bates, D. M. (2008). Mixed-effects modeling with crossed random effects for subjects and items. Journal of Memory and Language, 59(4), 390-412.

Baddeley, A. (2003). Working memory: Looking back and looking forward. Nature Reviews Neuroscience, 4(10), 829-839.

Baddeley, A., Gathercole, S., \& Papagno, C. (1998). The phonological loop as a language learning device. Psychological Review, 105(1), 158-173.

Barac, R., \& Bialystok, E. (2011). Cognitive development of bilingual children. Language Teaching, 44(1), 36-54.

Barnett, W. S., Yarosz, D. J., Thomas, J., Jung, K., \& Blanco, D. (2007). Two-way and monolingual English immersion in preschool education: An experimental comparison. Early Childhood Research Quarterly, 22(3), 277-293. 
Bhatia, T. K. \& Ritchie, W. C. (1999). The bilingual child: Some issues and perspectives. In W. C. Ritchie and T. K. Bhatia (Eds), Handbook of second language acquisition (pp. 569643). San Diego, CA: Academic Press.

Bhatia, T. K. \& Ritchie, W. C. (2004). Introduction. In T. K. Bhatia and W. C. Ritchie (Eds.), Handbook of bilingualism (pp. 1-2). Malden, MA: Blackwell Publishing.

Bialystok, E. (2001). Bilingualism in development: Language, literacy, and cognition. New York, NY: Cambridge University Press.

Bialystok, E. (2007). Language acquisition and bilingualism: Consequences for a multilingual society. Applied Psycholinguistics, 28(3), 393-397.

Bialystok, E., Luk, G., Peets, K. F., \& Yang, S. (2010). Receptive vocabulary differences in monolingual and bilingual children. Bilingualism, 1-7.

Bloom, P. (2000). How children learn the meaning of words. Cambridge, MA: MIT Press.

Bloom, P. (2001). Précis of how children learn the meanings of words. Behavioral and Brain Sciences, 24(6), 1095-1103.

Bonin, P., Peereman, R., Malardier, N., Meot, A., \& Chalard, M. (2003). A new set of 299 pictures for psycholinguistic studies: French norms for name agreement, image agreement, conceptual familiarity, visual complexity, image variability, age of acquisition, and naming latencies. Behavior Research Methods, Instruments, and Computers, 35(1), 158-167.

Brace, N., Kemp, R., \& Snelgar, R. (2009). SPSS for psychologists (4th ed.). London, UK: Palgrave Macmillan.

Brownell, R. (2000). Expressive One-Word Picture Vocabulary Test: Third Edition. Novato, CA: Academic Therapy Publications.

Carroll, J. B., \& White, M. N. (1973). Age-of-acquisition norms for 220 picturable nouns. Journal of Verbal Learning and Verbal Behavior, 12(5), 563-576.

Clark, E. V. (1993). The lexicon in acquisition. Cambridge, UK: Cambridge University Press.

Clark E. V. (2003). First language acquisition. Cambridge, NY: Cambridge University Press.

Clark, E. V., \& Hecht, B. F. (1983). Comprehension, production, and language acquisition. Annual Review of Psychology, 34, 325-349.

Cobo-Lewis, A.B., Pearson, B.Z., Eilers, R.E., \& Umbel, V.C. (2002). Effects of bilingualism and bilingual education on oral and written Spanish skills: A multifactor study of standardized test outcomes. In D.K. Oller \& R.E. Eilers (Eds.), Language and literacy in bilingual children (pp. 98-117). Clevedon: Multilingual Matters.

Conboy, B. T., \& Thal, D. J. (2006). Ties between the lexicon and grammar: Cross-sectional and longitudinal studies of bilingual toddlers. Child Development, 77(3), 712-735.

Crystal, D. (1997). English as a global language. Cambridge, UK: Cambridge University Press.

Cummins, J. (1979). Cognitive/academic language proficiency, linguistic interdependence, the optimum age question and some other matters. Working Papers on Bilingualism, No. 19, 121-129. 
Cummins, J. (1981). Age on arrival and immigrant second language learning in Canada: A reassessment. Applied Linguistics, 2(2), 132-149.

Cycowicz, Y. M., Friedman, D., Rothstein, M., \& Snodgrass, J. G. (1997). Picture naming by young children: Norms for name agreement, familiarity, and visual complexity. Journal of Experimental Child Psychology, 65(2), 171-237.

David, A., \& Wei, L. (2008). Individual differences in the lexical development of FrenchEnglish bilingual children. International Journal of Bilingual Education and Bilingualism, 11(5), 598-618.

De Avila, E. A., \& Duncan, S. E. (1981). Language Assessment Scales. San Rafael, CA: Linguametrics.

De Groot, A. M. B., \& Keijzer, R. (2000). What is hard to learn is easy to forget: The roles of word concreteness, cognate status, and word frequency in foreign-language vocabulary learning and forgetting. Language Learning, 50(1), 1-56.

Dickinson, D. K., McCabe, A., Anastasopoulos, L., Peisner-Feinberg, E. S., \& Poe, M. D. (2003). The comprehensive language approach to early literacy: The interrelationships among vocabulary, phonological sensitivity, and print knowledge among preschool-aged children. Journal of Educational Psychology, 95(3), 465-481.

Dunn, L. M., \& Dunn, L. M. (1981). Peabody Picture Vocabulary Test-Revised. Circle Pine, $\mathrm{MN}$ : American Guidance Service.

Dunn, L. M., \& Dunn, L. M. (1997). Peabody Picture Vocabulary Test-Third Edition. Circle Pines, MN: American Guidance Service.

Dunn, L. M., Padilla, E., Lugo, D., \& Dunn, L. M., (1986). Test de Vocabulario en Imagenes Peabody-Adaptacion Hispano-Americana [Peabody Picture Vocabulary Test-Latin American adaptation]. Circle Pines, MN: American Guidance Service.

Duran, L. K., Roseth, C. J., \& Hoffman, P. (2010). An experimental study comparing Englishonly and transitional bilingual education on Spanish-speaking preschoolers' early literacy development. Early Childhood Research Quarterly, 25(2), 207-217.

Duursma, E., Romero-Contreras, S., Szuber, A., Proctor, P., Snow, C., August, D., \& Calderon, M. (2007). The role of home literacy and language environment on bilinguals' English and Spanish vocabulary development. Applied Psycholinguistics, 28(1), 171-190.

Eilers, R. E., Oller, D. K., \& Cobo-Lewis, A. B. (2002). Bilingualism and cultural assimilation in Miami Hispanic children. In D. K. Oller \& R. E. Eilers (Eds.), Language and literacy in bilingual children (pp. 43-63). Clevedon, UK: Multilingual Matters.

Fan, M. (2000). How big is the gap and how to narrow it? An investigation into the active and passive vocabulary knowledge of L2 learners. RELC (Regional Language Centre) Journal, 31(2), 105-119.

Farnia, F., \& Geva, E. (2011). Cognitive correlates of vocabulary growth in English language learners. Applied Psycholinguistics, 32(4), 711-738.

Fenson, L., Dale, P. S., Reznick, J. S., Bates, E., Thal, D. J., \& Pethick, S. J. (1994). Variability in early communicative development. Monographs of the Society for Research in Child Development, 59(5), 1-173. 
Fernald, A. (2006). When infants hear two languages: Interpreting research on early speech perception by bilingual children. In P. McCardle and E. Hoff (Eds), Childhood bilingualism: Research on infancy through school age (pp. 19-29). Buffalo, NY: Multilingual Matters.

Feuerverger, G. (1991). University students' perceptions of heritage language learning and ethnic identity maintenance. Canadian Modern Language Review, 47(4), 660-677.

Fromkin, V. A. (1987). The lexicon: Evidence from acquired dyslexia. Language, 63(1), 1-22.

Galobardes, B., Lynch, J., \& Smith, G. D. (2007). Measuring socioeconomic position in health research. British Medical Bulletin, 81-82, 21-37.

Garcia. E. E. (1983). Early childhood bilingualism: With special reference to the MexicanAmerican child. Albuquerque, NM: University of New Mexico Press.

Gardner, M. F. (1990). Expressive One-Word Picture Vocabulary Test-Revised. Novato, CA: Academic Therapy Publications.

Gass, S. M., \& Selinker L. (1994). Second language acquisition: An introductory course. Hillsdale, NJ: Lawrence Erlbaum Associates.

Gathercole, S. E. (2006). Nonword repetition and word learning: The nature of the relationship. Applied Psycholinguistics, 27(4), 513-543.

Gathercole, S. E., \& Baddeley, A. D. (1989). Evaluation of the role of phonological STM in the development of vocabulary in children: A longitudinal study. Journal of Memory and Language, 28(2), 200-213.

Gathercole, S. E., Hitch, G. J., Service, E., \& Martin, A. J. (1997). Phonological short-term memory and new word learning in children. Developmental Psychology, 33(6), 966-979.

Gathercole, V. C. M., Thomas, E. M., \& Hughes, E. (2008). Designing a normed receptive vocabulary test for bilingual populations: A model from Welsh. International Journal of Bilingual Education and Bilingualism, 11(6), 678-720.

Genesee, F. (2006). Bilingual first language acquisition in perspective. In P. McCardle and E. Hoff (Eds.), Childhood bilingualism: Research on infancy through school age (pp. 4567). Buffalo, NY: Multilingual Matters.

Genesee, F., Nicoladis, E., \& Paradis, J. (1995). Language differentiation in early bilingual development. Journal of Child Language, 22(October), 611-631.

Genesee, F., Paradis J., \& Crago M. B. (2004). Dual language development and disorders: A handbook on bilingualism and second language learning. Baltimore, MD: Paul H. Brookes.

Golberg, H., Paradis, J., \& Crago, M. (2008). Lexical acquisition over time in minority first language children learning English as a second language. Applied Psycholinguistics, 29(1), 41-65.

Goldfield, B. A., \& Reznick, J. S. (1990). Early lexical acquisition: Rate, content, and the vocabulary spurt. Journal of Child Language, 17(1), 171-183.

Goldstein, B. A. (2006). Clinical implications of research on language development and disorders in bilingual children. Topics in Language Disorders, 26(4), 305-321. 
Gonzalez, V. (2001). The role of socioeconomic and sociocultural factors in language minority children's development: An ecological research view. Bilingual Research Journal, 25(12), 1-30.

Grosjean, F. (1982). Life with two languages: An introduction to bilingualism. Cambridge, MA: Harvard University Press.

Guardado, M. (2002). Loss and maintenance of first language skills: Case studies of Hispanic families in Vancouver. Canadian Modern Language Review, 58(3), 341-363.

Gutiérrez-Clellen, V. F., \& Kreiter, J. (2003). Understanding child bilingual acquisition using parent and teacher reports. Applied Psycholinguistics, 24(2), 267-288.

Hagopian, G. (2005). Armenian for everyone: Western and Eastern Armenian in parallel lessons. Ann Arbor: Caravan Books.

Hakuta, K., \& D'Andrea, D. (1992). Some properties of bilingual maintenance and loss in Mexican background high-school students. Applied Linguistics, 13(1), 72-99.

Hammer, C. S., Lawrence, F. R., \& Miccio, A. W. (2007). Bilingual children's language abilities and early reading outcomes in Head Start and Kindergarten. Language, Speech, and Hearing Services in Schools, 38(3), 237-248.

Hart, B. \& Risley, T. (1995). Meaningful differences in the everyday experience of young American children. Baltimore, MD: Brookes Publishing.

Hesbacher, P., \& Fishman, J. A. (1964). Language loyalty: Its functions and concomitants in two bilingual communities. Lingua, 13(C), 145-165.

Hoff, E. (2005). Language development (3rd ed.). Belmont, CA: Wadsworth/Thomson Learning.

Hoff-Ginsberg, E. (1994). Influences of mother and child on maternal talkativeness. Discourse Processes, 18(1), 105-117.

Hoff, E., \& Naigles, L. (2002). How children use input to acquire a lexicon. Child Development, 73(2), 418-433.

Hurtado, N., Marchman, V. A., \& Fernald, A. (2008). Does input influence uptake? Links between maternal talk, processing speed and vocabulary size in Spanish-learning children. Developmental Science, 11(6), F31-F39.

Huttenlocher, J., Haight, W., Bryk, A., Seltzer, M., \& Lyons, T. (1991). Early vocabulary growth: Relation to language input and gender. Developmental Psychology, 27(2), 236248.

Kan, P. F., \& Kohnert, K. (2005). Preschoolers learning Hmong and English: Lexical-semantic skills in L1 and L2. Journal of Speech, Language, and Hearing Research, 48(2), 372383.

Kaprielian-Churchill, I. (2005). Like Our Mountains: A History of Armenians in Canada. Montreal: McGill-Queen's University Press.

Kohnert, K. (2002). Picture naming in early sequential bilinguals: A 1-year follow-up. Journal of Speech, Language, and Hearing Research, 45(4), 759-771. 
Kohnert, K. J., \& Bates, E. (2002). Balancing bilinguals II: Lexical comprehension and cognitive processing in children learning Spanish and English. Journal of Speech, Language, and Hearing Research, 45(2), 347-359.

Kohnert, K. J., Bates, E., \& Hernandez, A. E. (1999). Balancing bilinguals: Lexical-semantic production and cognitive processing in children learning Spanish and English. Journal of Speech, Language, and Hearing Research, 42(6), 1400-1413.

Kohnert, K., Yim, D., Nett, K., Kan, P. F., \& Duran, L. (2005). Intervention with linguistically diverse preschool children: A focus on developing home language(s). Language, Speech, and Hearing Services in Schools, 36(3), 251-263.

Lambert, W. E. (1977). Effects of bilingualism on the individual: Cognitive and sociocultural consequences. In P.A. Hornby (Ed.), Bilingualism: Psychological, social and educational implications (pp. 15-28). New York, NY: Academic Press.

Lambert, W. E. (1981). Bilingualism and language acquisition. In H. Winitz (Ed.), Native language and foreign language acquisition (pp. 9-22). New York, NY: Academy of Science.

Leseman, P. P. M. (2000). Bilingual vocabulary development of Turkish preschoolers in the Netherlands. Journal of Multilingual and Multicultural Development. 21(2), 93-112.

Lindsey, K. A., Manis, F. R., \& Bailey, C. E. (2003). Prediction of first-grade reading in Spanish-speaking English-language learners. Journal of Educational Psychology, 95(3), 482-494.

Louis, W. \& Taylor, D. M. (2001). When the survival of a language is at stake: The future of Inuktitut in arctic Québec. Journal of Language and Social Psychology, 20(1-2), 111143.

Luo, S. H., \& Wiseman, R. L. (2000). Ethnic language maintenance among Chinese immigrant children in the United States. International Journal of Intercultural Relations, 24(3), 307324.

Macnamara, J. (1966). Bilingualism and primary education: A study of Irish experience. Edinburgh, UK: Edinburgh University Press.

Malmkjær, K. (Ed.) (2002). The linguistics encyclopedia (2nd ed.). New York, NY: Routledge.

Marchman, V. A., Martinez-Sussmann, C., \& Dale, P. S. (2004). The language-specific nature of grammatical development: Evidence from bilingual language learners. Developmental Science, 7(2), 212-224.

MacDonald, M. C., \& Christiansen, M. H. (2002). Reassessing working memory: Comment on Just and Carpenter (1992) and Waters and Caplan (1996). Psychological Review, 109(1), $35-54$.

McCardle, P. D., \& Hoff, E. (2006). An agenda for research on childhood bilingualism. In P. D. McCardle \& E. Hoff (Eds.), Childhood bilingualism: Research on infancy through school age (pp. 157-165). Buffalo, NY: Multilingual Matters.

McCardle, P., Kim, J., Grube, C., \& Randall, V. (1995). An approach to bilingualism in early intervention. Infants and Young Children, 7(3), 63-73. 
Meyer, A. S., Roelofs, A., \& Levelt, W. J. M. (2003). World length effects in object naming: The role of a response criterion. Journal of Memory and Language, 48(1), 131-147.

Miller, P., \& Hoogstra, L. (1992). Language as a tool in the socialization and apprehension of cultural meanings. In T. Schwartz, G. White and C. Lutz (Eds.), New directions in psychological anthropology (pp. 85-101). Cambridge, UK: Cambridge University Press.

Morrison, C. M., Chappell, T. D., \& Ellis, A. W. (1997). Age of acquisition norms for a large set of object names and their relation to adult estimates and other variables. Quarterly Journal of Experimental Psychology Section A: Human Experimental Psychology, 50(3), 528-559.

Morrison, C. M., Ellis, A. W., \& Quinlan, P. T. (1992). Age of acquisition, not word frequency, affects object naming, not object recognition. Memory and Cognition, 20(6), 705-714.

Nation, I. S. P. (1990). Teaching and learning vocabulary. Boston, MA: Heinle \& Heinle Publishers.

Nation, I. S. P. (2001). Learning vocabulary in another language. New York: Cambridge University Press.

Nicoladis, E., \& Genesee, F. (1996). Word awareness in second language learners and bilingual children. Language Awareness, 5(2), 80-90.

Obied, V. M. (2009). How do siblings shape the language environment in bilingual families? International Journal of Bilingual Education and Bilingualism, 12(6), 705-720.

Oller, D. K., \& Eilers, R. E. (2002). Language and literacy in bilingual children. Clevedon: Multilingual Matters.

Oller, D. K., Eilers, R. E., Urbano, R., \& Cobo-Lewis, A. B. (1997). Development of precursors to speech in infants exposed to two languages. Journal of Child Language, 24(2), 407425.

Oller, D.K., \& Pearson, B. Z. (2002). Assessing the effects of bilingualism: A background, In D. K. Oller \& R. E. Eilers (Eds.), Language and literacy in bilingual children (pp. 3-21). Clevedon: Multilingual Matters.

Orellana, M. F. (1994). Appropriating the voice of the superheroes: Three preschoolers' bilingual language uses in play. Early Childhood Research Quarterly, 9(2), 171-193.

Paradis, J., \& Genesee, F. (1996). Syntactic acquisition in bilingual children: Autonomous or interdependent? Studies in Second Language Acquisition, 18(March), 1-25.

Paradis, J., \& Genesee, F. (1997). On continuity and the emergence of functional categories in bilingual first-language acquisition. Language Acquisition: A Journal of Developmental Linguistics, 6(2), 91-124.

Parra, M., Hoff, E., \& Core, C. (2011). Relations among language exposure, phonological memory, and language development in Spanish-English bilingually developing 2-yearolds. Journal of Experimental Child Psychology, 108(1), 113-125.

Patterson, J. L. (2002). Relationships of expressive vocabulary to frequency of reading and television experience among bilingual toddlers. Applied Psycholinguistics, 23(4), 493508. 
Paul, R. (2001). Language disorders from infancy through adolescence: Assessment \& intervention (2nd ed.). St. Louis, MO: Mosby.

Peal, E., \& Lambert, W. E. (1962). The relation of bilingualism to intelligence. Psychological Monographs, 76(27, Whole No.546), 1-23.

Pearson, B. Z. (2007). Social factors in childhood bilingualism in the United States. Applied Psycholinguistics, 28(3), 399-410.

Pearson, B. Z., Fernandez, S. C., Lewedeg, V., \& Oller, D. K. (1997). The relation of input factors to lexical learning by bilingual infants. Applied Psycholinguistics, 18(1), 41-58.

Pearson, B. Z., Fernandez, S. C., \& Oller, D. K. (1993). Lexical development in bilingual infants and toddlers: Comparison in monolingual terms. Language Learning, 43(1), 93-120.

Pearson, B. Z., Fernandez, S. C., \& Oller, D. K. (1995). Cross-language synonyms in the lexicons of bilingual infants: One language or two? Journal of Child Language, 22(2), 345-368.

Pennington, L., James, P., McNally, R., Pay, H., \& McConachie, H. (2009). Analysis of compositional data in communication disorders research. Journal of Communication Disorders, 42(1), 18-28.

Petitto, L. A., Katerelos, M., Levy, B. G., Gauna, K., Tetreault, K., \& Ferraro, V. (2001). Bilingual signed and spoken language acquisition from birth: Implications for the mechanisms underlying early bilingual language acquisition. Journal of Child Language, 28(2), 453-496.

Portney, L. G., \& Watkins, M. P. (2000). Foundations of clinical research: Applications to practice (2nd ed.). Upper Saddle River, NJ: Prentice Hall Health.

Raaijmakers, J. G. W., Schrijnemakers, J. M. C., \& Gremmen, F. (1999). How to deal with "The language-as-fixed-effect fallacy": Common misconceptions and alternative solutions. Journal of Memory and Language, 41(3), 416-426.

Rice, M. L., Huston, A. C., Truglio, R., \& Wright, J. (1990). Words from "Sesame Street": Learning vocabulary while viewing. Developmental Psychology, 26(3), 421-428.

Rodriguez, J. L., Diaz, R. M., Duran, D., \& Espinosa, L. (1995). The impact of bilingual preschool education on the language development of Spanish-speaking children. Early Childhood Research Quarterly, 10(4), 475-490.

Roid, G. H., \& Miller, L. J. (1995, 1997). Leiter International Performance Scale-Revised. Wood Dale, IL: Stoelting.

Roid, G. H., \& Miller, L. J. (1997). Leiter International Performance Scale-Revised: Examiner's Manual. In G. H. Roid \& L. J. Miller, Leiter International Performance Scale-Revised. Wood Dale, IL: Stoelting.

Sakayan, D. (2000). Modern Western Armenian for the English-speaking world: A contrastive approach. Montreal, Quebec, Canada: Arod Books.

Samuelian, T. J. (1993). Armenian dictionary in transliteration: Western pronunciation: Armenian-English, English-Armenian. New York, NY: Armenian National Education Committee. 
Scheele, A. F., Leseman, P. P. M., \& Mayo, A. Y. (2010). The home language environment of monolingual and bilingual children and their language proficiency. Applied Psycholinguistics, 31(1), 117-140.

Service, E. (1992). Phonology, working memory, and foreign-language learning. Quarterly Journal of Experimental Psychology Section A: Human Experimental Psychology, 45(1), 21-50.

Sheng, L., Lu, Y., \& Kan, P. F. (2011). Lexical development in Mandarin-English bilingual children. Bilingualism, 1-9.

Snodgrass, J. G., \& Vanderwart, M. (1980). A standardized set of 260 pictures: Norms for name agreement, image agreement, familiarity, and visual complexity. Journal of Experimental Psychology Human Learning and Memory, 6(2), 174-215.

Stadthagen-Gonzalez, H., \& Davis, C. J. (2006). The Bristol norms for age of acquisition, imageability, and familiarity. Behavior Research Methods, 38(4), 598-605.

Statistics Canada. (2001). Canada's ethnocultural portrait: The changing mosaic. Retrieved January 8, 2007 from

http://www12.statcan.ca/english/census01/products/analytic/companion/etoimm/canada.c $\underline{\mathrm{fm}}$

Statistics Canada. (2007). Population by mother tongue and age groups, percentage distribution (2006), for Canada, provinces and territories - 20\% sample data. Statistics Canada, December 4, 2007. 2006 Census of Canada. Catalogue number 97-555-XWE2006002.

Statistics Canada. (2010). Detailed mother tongue (103), language sSpoken most often at home (8), other language spoken regularly at home (9) and age groups (17A) for the population of Canada, provinces, territories, census metropolitan areas and census agglomerations, 2006 Census - 20\% sample data. Statistics Canada, January 6, 2010. 2006 Census of Canada. Catalogue number 97-555-XCB2006028.

Szekely, A., Jacobsen, T., D'Amico, S., Devescovi, A., Andonova, E., Herron, D., et al. (2004). A new on-line resource for psycholinguistic studies. Journal of Memory and Language, 51(2), 247-250.

Thordardottir, E. T. (2005). Early lexical and syntactic development in Quebec French and English: Implications for cross-linguistic and bilingual assessment. International Journal of Language and Communication Disorders, 40(3), 243-278.

Thordardottir, E. T., \& Weismer, S. E. (1996). Language assessment via parent report: Development of a screening instrument for Icelandic children. First Language, 16(48), 265-285.

Tomasello, M. (2003). Constructing a language: A usage-based theory of language acquisition. Cambridge, MA: Harvard University Press.

Umbel, V. M., Pearson, B. Z., Fernandez, M. C., \& Oller, D. K. (1992). Measuring bilingual children's receptive vocabularies. Child Development, 63(4), 1012-1020.

Van der Schuit, M., Segers, E., Van Balkom, H., \& Verhoeven, L. (2011). How cognitive factors affect language development in children with intellectual disabilities. Research in Developmental Disabilities, 32(5), 1884-1894. 
Volterra, V., \& Taeschner, T. (1978). The acquisition and development of language by bilingual children. Journal of Child Language, 5(2), 311-326.

Webb, S. (2008). Receptive and productive vocabulary sizes of L2 learners. Studies in Second Language Acquisition, 30(1), 79-95.

Wechsler, D. (1991). Wechsler Intelligence Scale for Children (3rd ed.). San Antonio, TX: The Psychological Corporation.

Williams, K. T. (1997). Expressive Vocabulary Test. Circle Pines, MN: American Guidance Service.

Winsler, A., Diaz, R. M., Espinosa, L., \& Rodriguez, J. L. (1999). When learning a second language does not mean losing the first: Bilingual language development in low-income, Spanish-speaking children attending bilingual preschool. Child Development, 70(2), 349362.

Wong Fillmore, L. (1991). When learning a second language means losing the first. Early Childhood Research Quarterly, 6(3), 323-346. 


\section{Appendix A}

\section{Written Permission for Using Pictures}

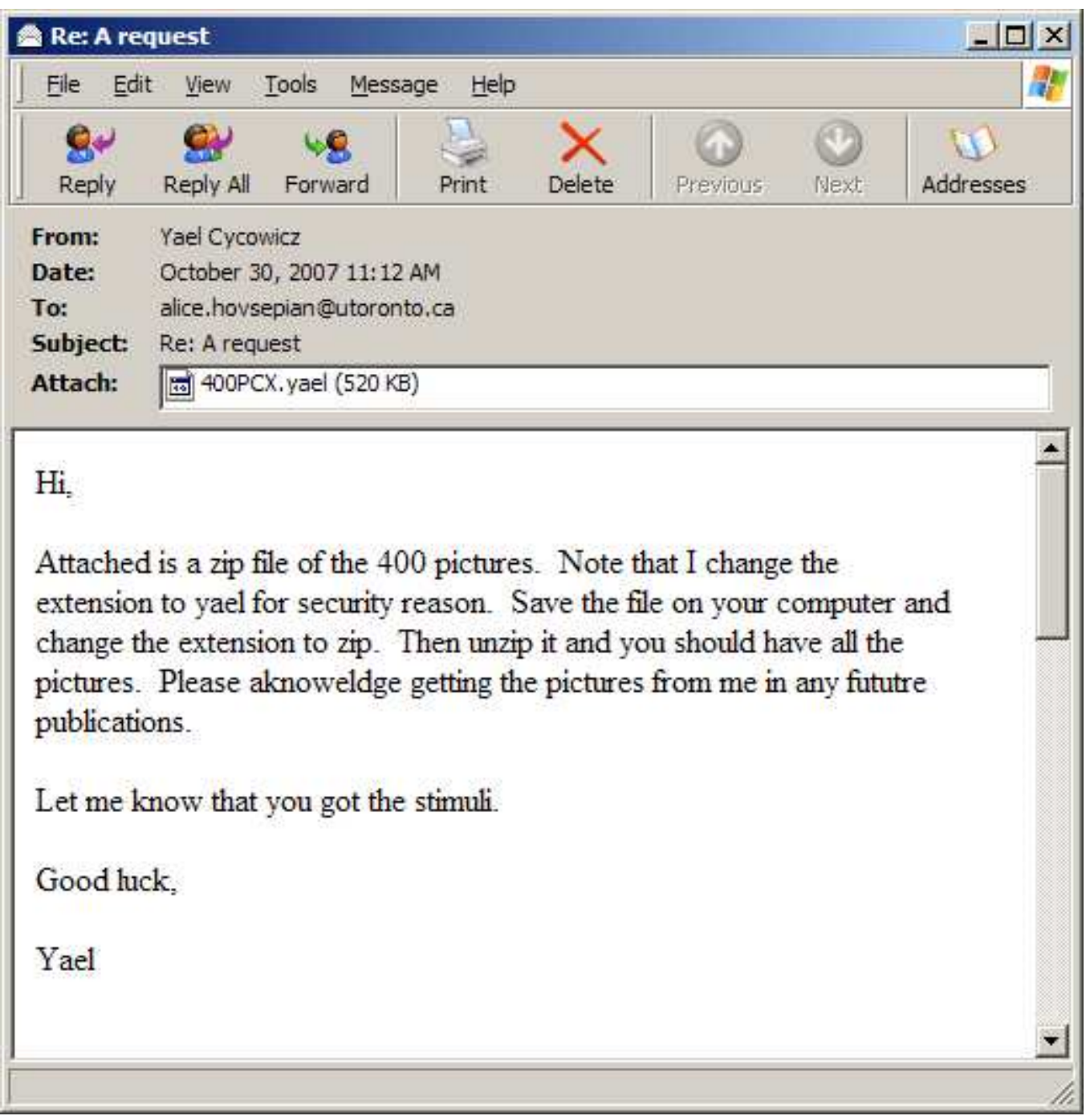




\begin{tabular}{|c|c|c|c|c|c|}
\hline \multicolumn{5}{|c|}{ Q RE: New User - Central European (ISO) } & \multirow{3}{*}{$\frac{-1 ㅁ ㅣ}{A y}$} \\
\hline \multicolumn{5}{|c|}{ Eile Edit View Iools Message Help } & \\
\hline Reply & $\underbrace{}_{\text {Reply All }}=8$ & $\underset{\text { Print }}{\mathrm{y}} \underset{\text { Delete }}{\mathrm{X}}$ & $\underset{\text { Previous: }}{(1)}$ & Addresses & \\
\hline \multicolumn{6}{|c|}{$\begin{array}{ll}\text { From: } & \text { Anna Szekely } \\
\text { Date: } & \text { November } 21,20076: 20 \text { AM } \\
\text { To: } & \text { alice.hovsepian@utoronto.ca } \\
\text { Subject: } & \text { RE: New User }\end{array}$} \\
\hline \multicolumn{5}{|c|}{ Dear Alice! } & \\
\hline \multicolumn{5}{|c|}{$\begin{array}{l}\text { You are most welcome to access our on-line materials with a password on } \\
\text { a lab-to-lab basis as explained in full detail below for RESEARCH } \\
\text { PURPOSES ONLY, with an understanding that you CANNOT REPUBLISH any of } \\
\text { the pictures that are NOT open for public use (password protected). } \\
\text { However, before we send you the login name and password could you please } \\
\text { ask your tutor for verification and identification of your request (I } \\
\text { would need an email from your tutor with clear identification of the } \\
\text { project and lab for a record of where the pictures go). }\end{array}$} & \\
\hline
\end{tabular}

Continued on next page 


\begin{tabular}{|c|c|c|c|c|c|c|}
\hline \multicolumn{6}{|c|}{ Q RE: New User - Central European (ISO) } & \multirow{3}{*}{$\frac{-|\square| x \mid}{2 y}$} \\
\hline Eile Edit & View & Iools $\mathrm{Me}$ & \multicolumn{3}{|c|}{ Message Help } & \\
\hline Reply & Reply All & Forward & $\underset{\text { Print }}{X}$ & Previous & Addresses & \\
\hline \multicolumn{7}{|c|}{$\begin{array}{ll}\text { From: } & \text { Anna Szekely } \\
\text { Date: } & \text { November } 21,20076: 20 \text { AM } \\
\text { To: } & \text { alice.hovsepian@utoronto.ca } \\
\text { Subject: } & \text { RE: New User }\end{array}$} \\
\hline \multicolumn{6}{|c|}{$\begin{array}{l}\text { DETAIIS: } \\
\text { You may access to the full set of pictures and database, with an } \\
\text { understanding that the pictures that we have used for all of these } \\
\text { studies are a compendium of drawings from various sources: } \\
\text { http:/crl.ucsd.edu/ aszekely ipnp/sources.html including the originall } \\
\text { Snodgrass pictures (a source which is NOT OPEN for public use), a } \\
\text { published (and openly available) series by Abbatte \& Chappelle, and } \\
\text { other sources including the Boston Naming Test and the Peabody Picture } \\
\text { Vocabulary Test. So obviously these aren't 'ours' to distribute } \\
\text { publicly. Most of them are available for free to researchers, but we } \\
\text { can't republish them ourselves without permis sion, and cannot put them } \\
\text { up on the Internet. We have asked all of the sources for permission to } \\
\text { put the full set on our website when we published several big papers on } \\
\text { our International Picture-Naming Project, but unfortunately for some of } \\
\text { the pictures we have not obtained web-permission. What we have done, } \\
\text { however, is to share the digitized picture files that we use (520 object } \\
\text { pictures; } 275 \text { action pictures) with individual researchers on a personal } \\
\text { basis (from our lab to their lab), we ask researchers to identify } \\
\text { themselves with some details of their project and lab (for a record of } \\
\text { where the pictures go). And naturally any information calculated from } \\
\text { the database should be referred to by quoting the relevant articles } \\
\text { (see: } \text { http:/crl.ucsd.edu/ aszekely /ipnp/1references.html for updated } \\
\text { details). }\end{array}$} & $\Delta$ \\
\hline \multicolumn{7}{|c|}{$\begin{array}{l}\text { All the best, } \\
\text { Anna }\end{array}$} \\
\hline \multicolumn{7}{|c|}{ Anna Szekely Ph.D. (Associate Professor) } \\
\hline \multicolumn{7}{|c|}{$\begin{array}{l}\text { Institute of Psychology, Eötvös Loránd University, Budapest } \\
\text { Izabella u. } 46 \text {, Budapest, Hungary-1064 } \\
\text { Email: anna } \alpha \text { crl.ucsd.edu, anna } a \text { puskin.sote.hu } \\
\text { Website: } \underline{\text { http//www.crl.ucsd.edu/ aszekely/ }} \\
\text { Phone: }(36-1) 461-2691 \\
\text { Fax: (36-1) } 461-2691\end{array}$} \\
\hline
\end{tabular}


in

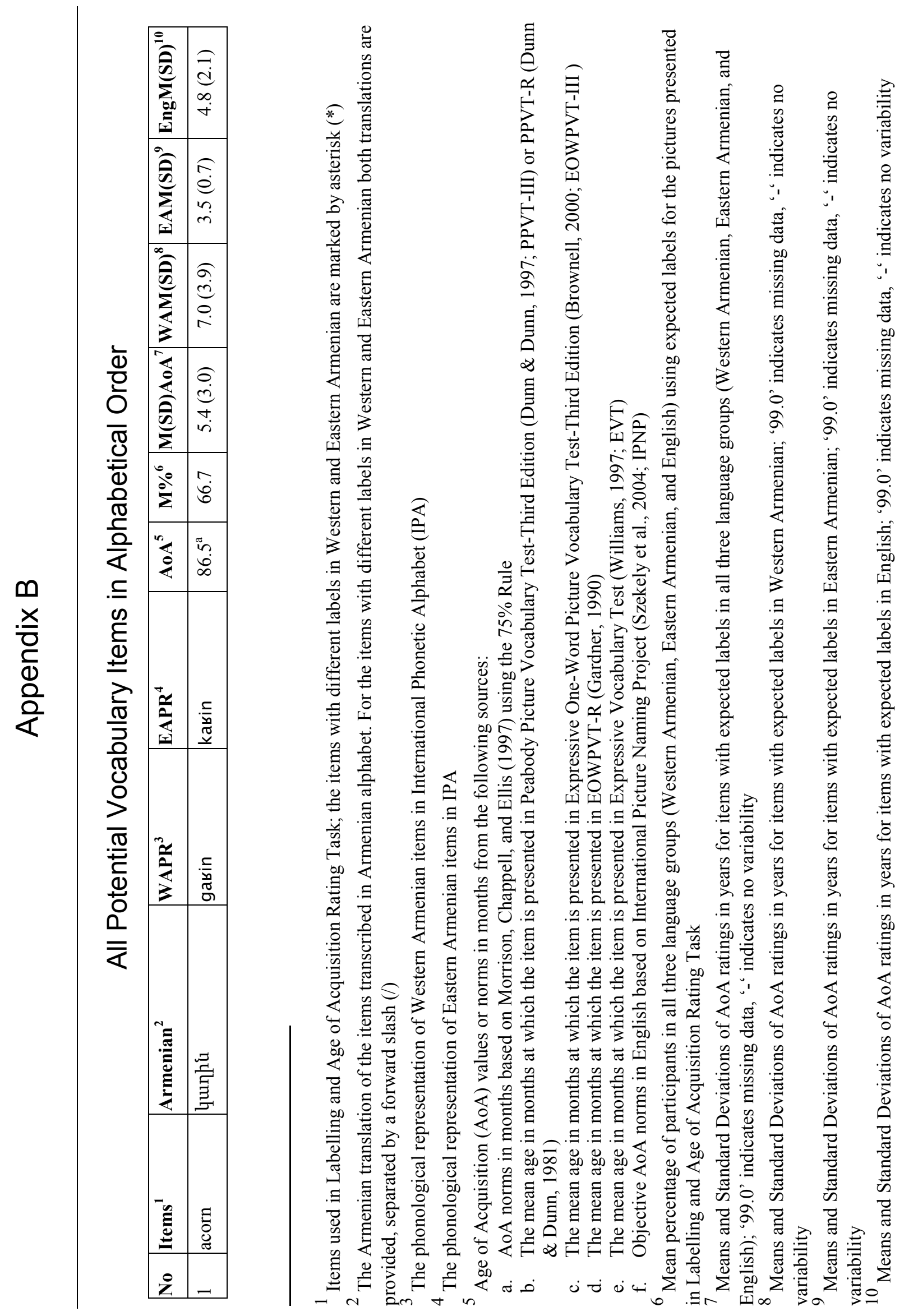




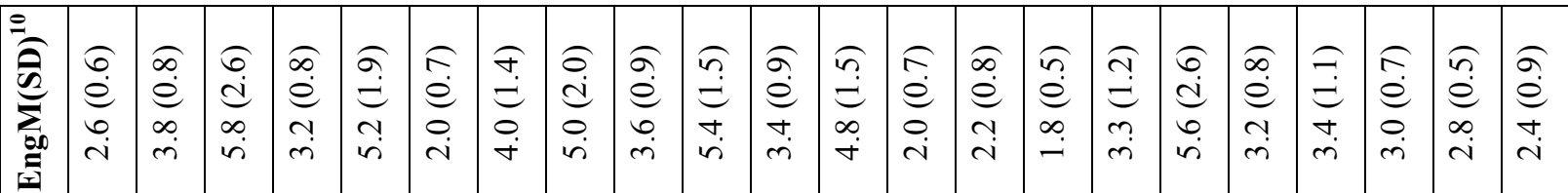

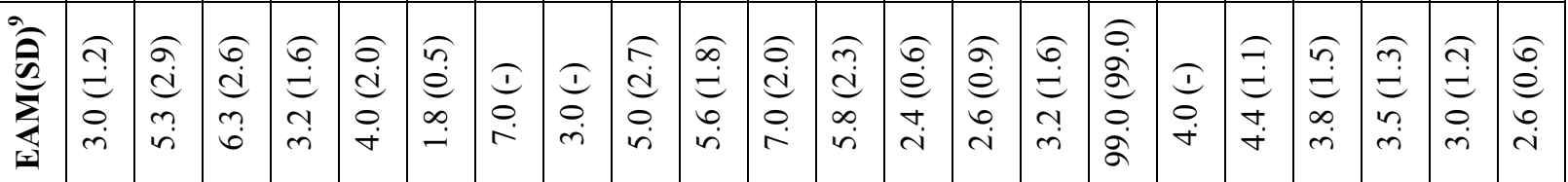

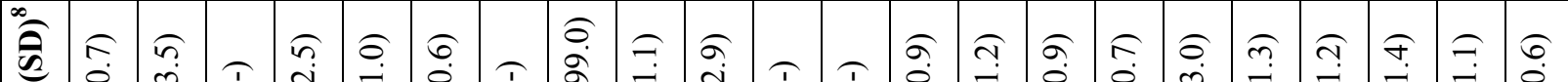

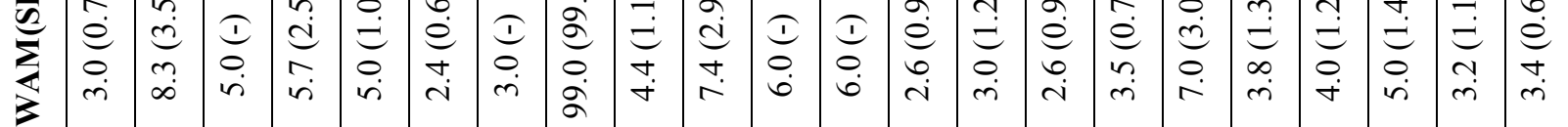

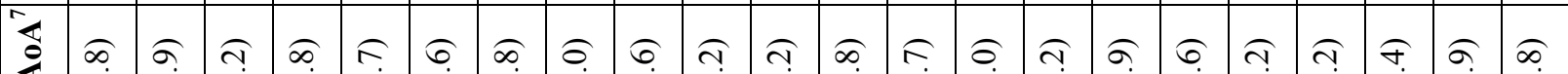

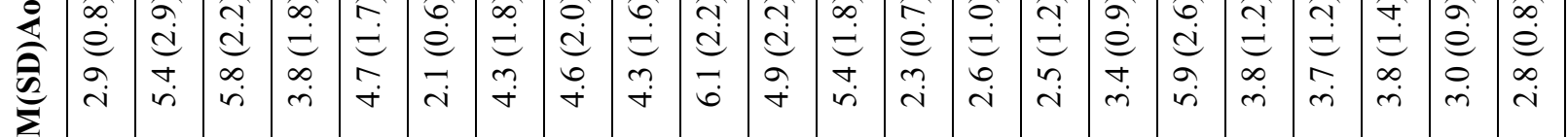

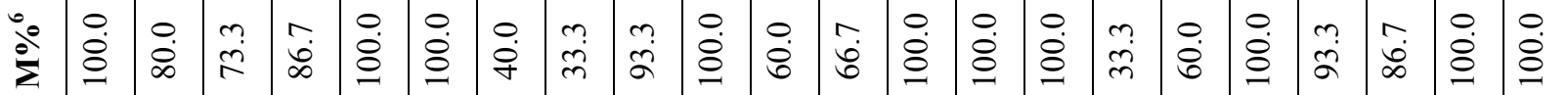

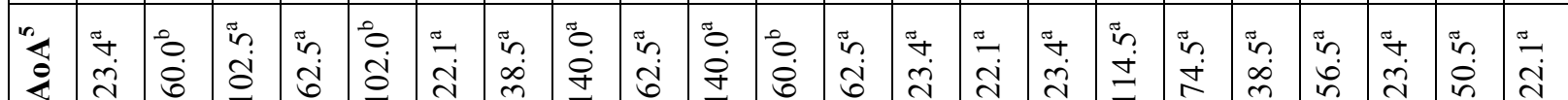

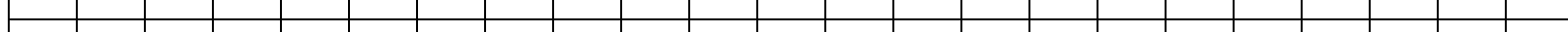

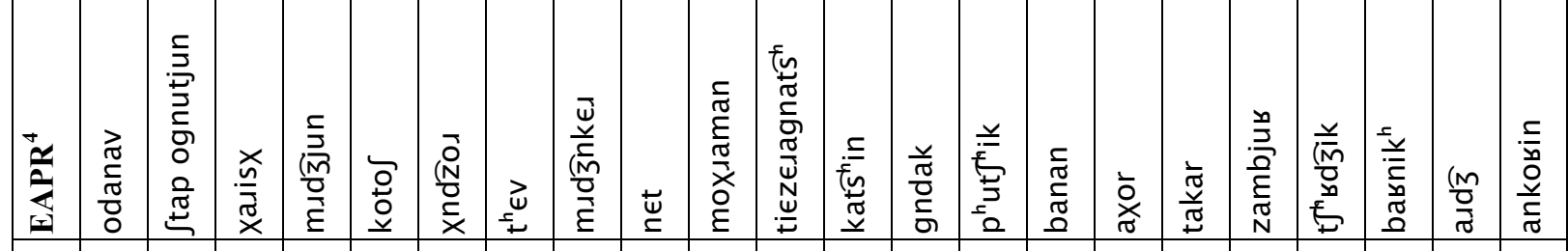

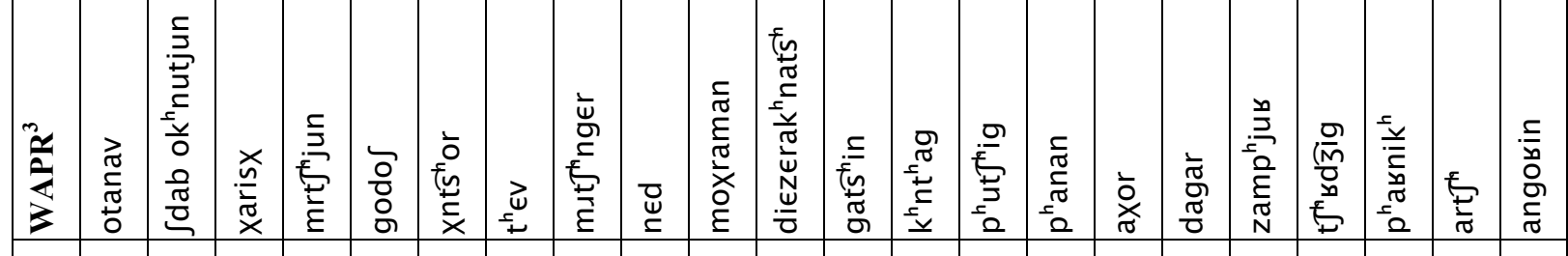

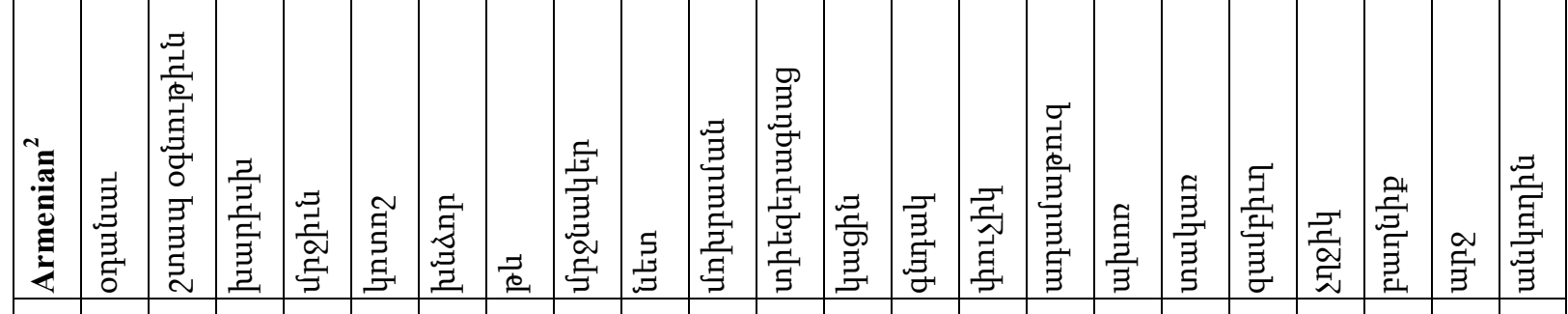

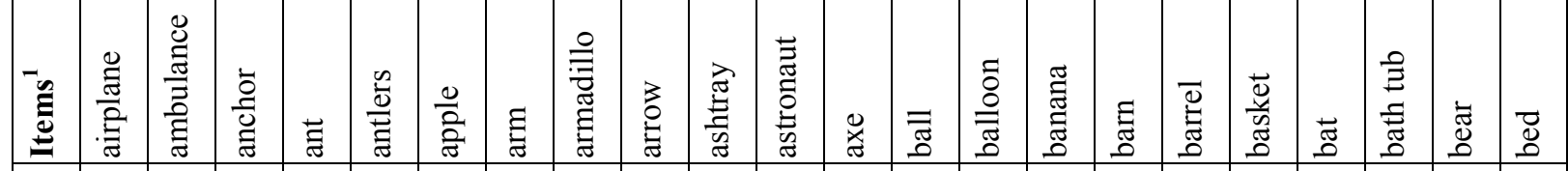

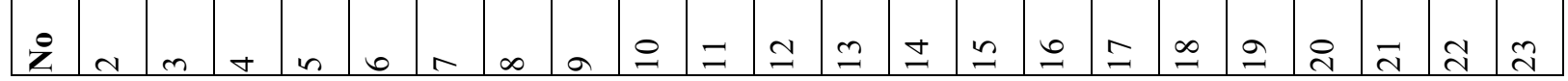


in

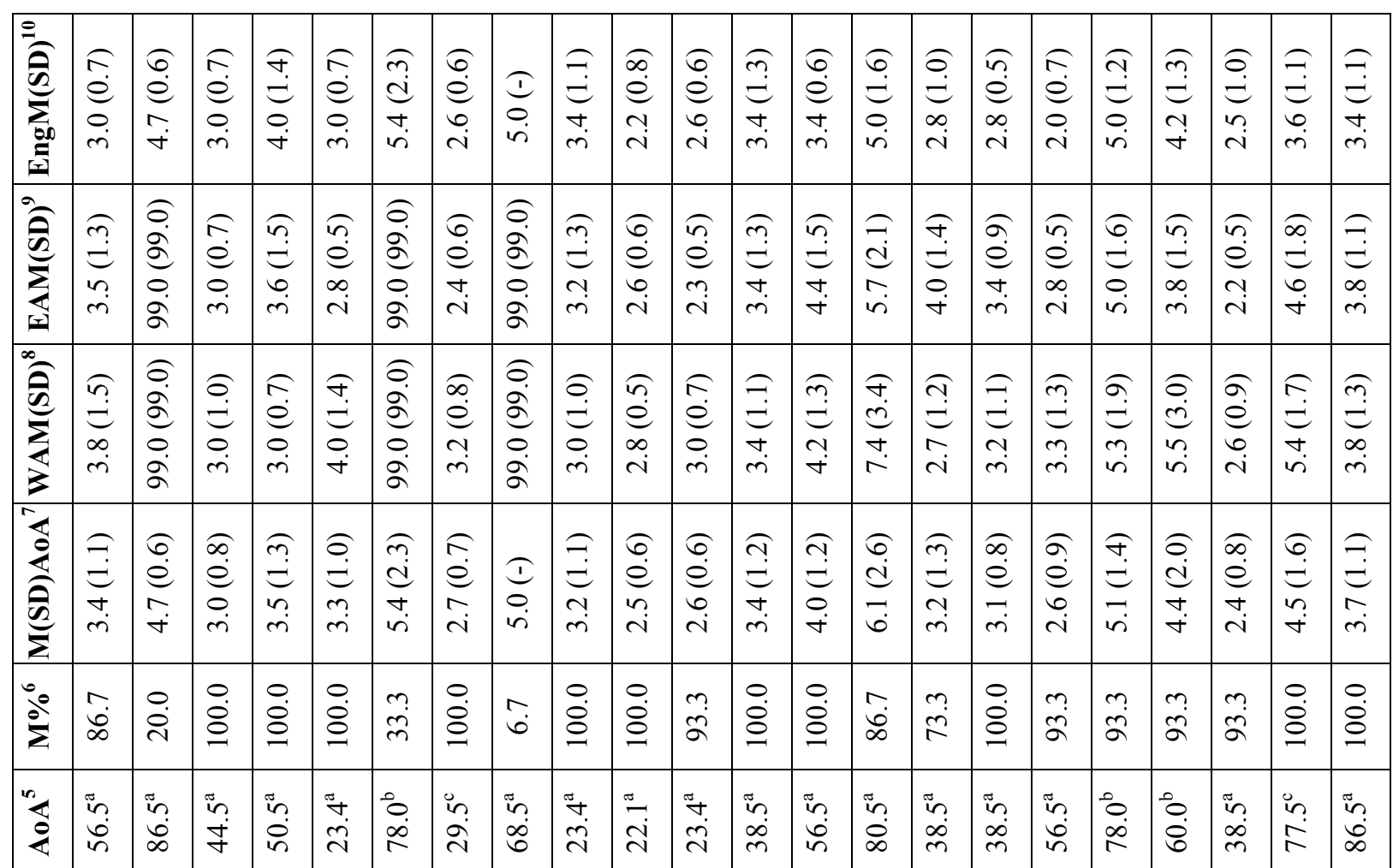

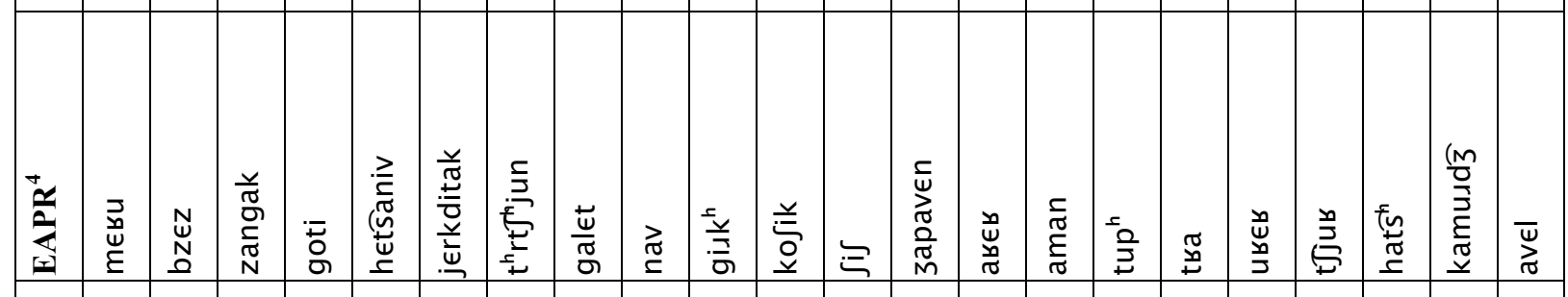

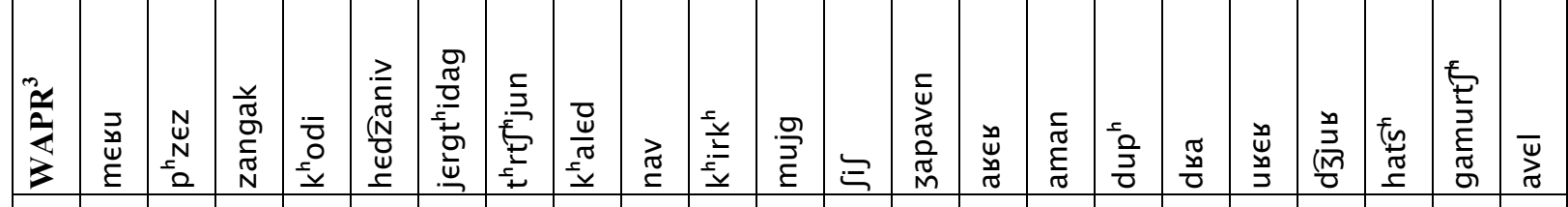

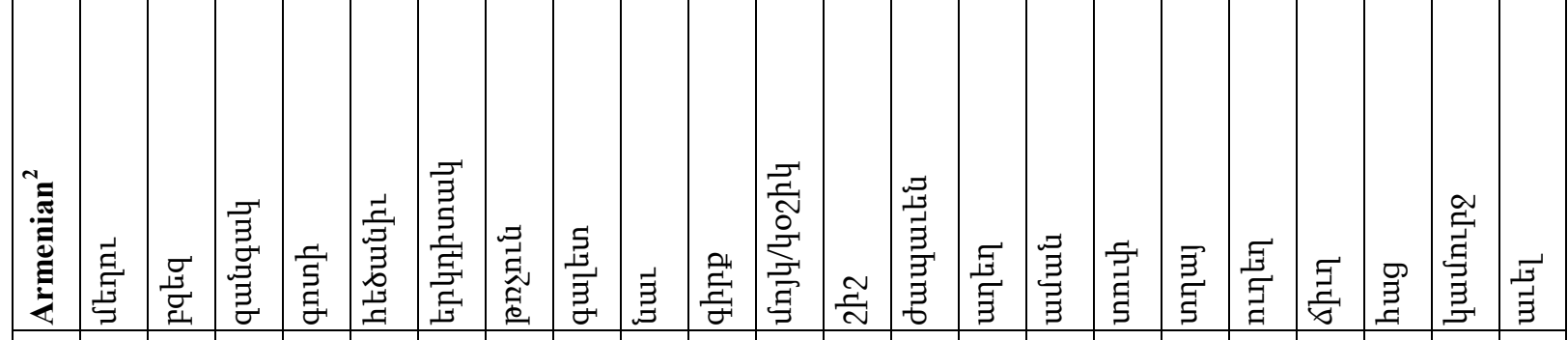

\begin{tabular}{|c|c|c|c|c|c|c|c|c|c|c|c|c|c|c|c|c|c|c|c|c|c|c|}
\hline 次 & $\mathbb{0}$ & 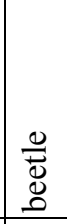 & $\overline{0}$ & 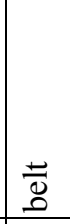 & \begin{tabular}{|l}
$\frac{0}{0}$ \\
$\frac{0}{0}$ \\
\end{tabular} & 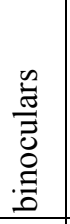 & ت. & 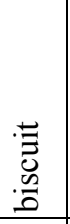 & $\begin{array}{l}\overrightarrow{0} \\
8 \\
\end{array}$ & $\begin{array}{l}y \\
\\
8 \\
\end{array}$ & $\begin{array}{l}* \\
\end{array}$ & 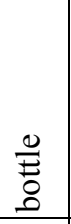 & 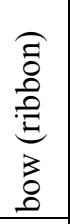 & 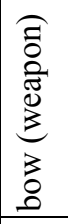 & \begin{tabular}{|l}
3 \\
\\
\end{tabular} & ஷ̊ & 官 & 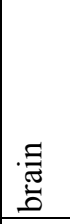 & 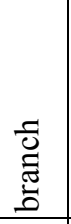 & 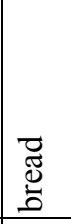 & 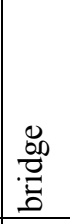 & $\begin{array}{r}\Xi \\
0 \\
0 \\
0\end{array}$ \\
\hline$\ddot{z}$ & $\stackrel{d}{d}$ & $\approx$ & 루 & $\hat{\imath}$ & $\underset{\sim}{\infty}$ & Әे & ণ & $\bar{m}$ & 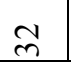 & $m$ & m & $n$ & r & $n$ & $\underset{m}{\infty}$ & ले & 웅 & 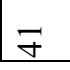 & Y & $\mathscr{F}$ & \& & 年 \\
\hline
\end{tabular}


$\stackrel{\infty}{2}$

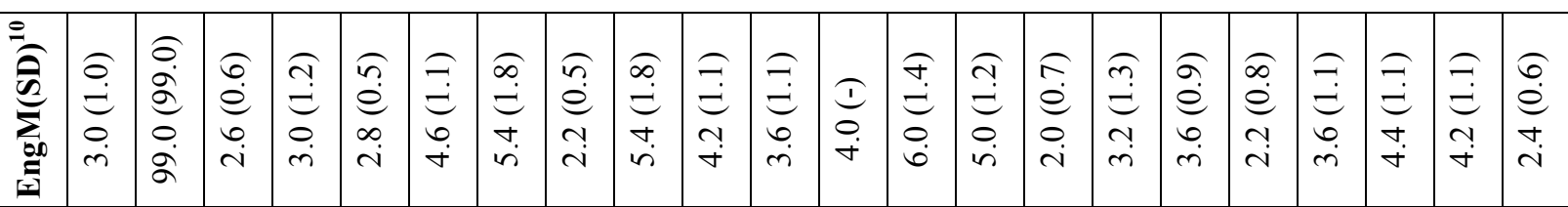

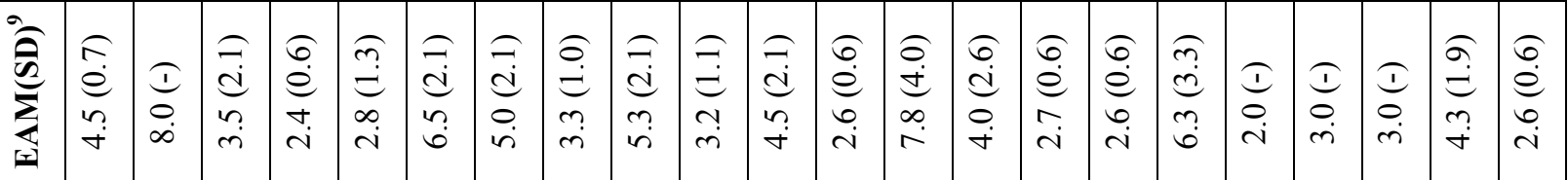
商

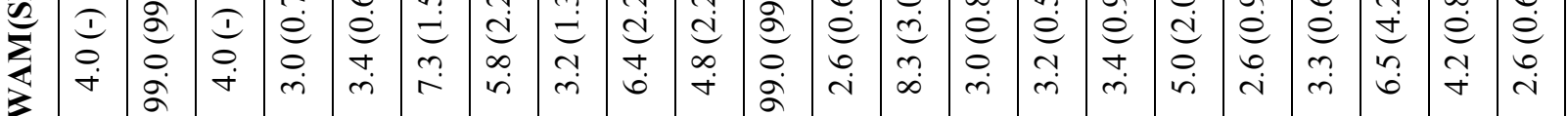

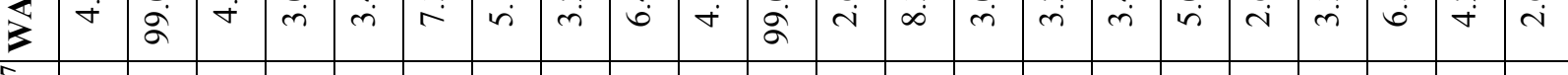

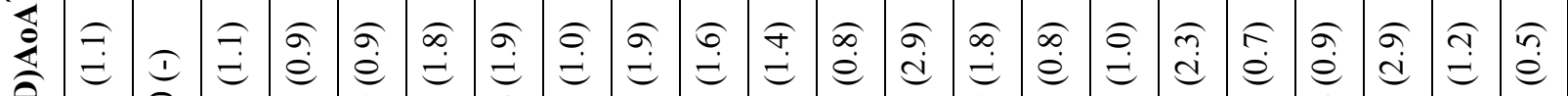

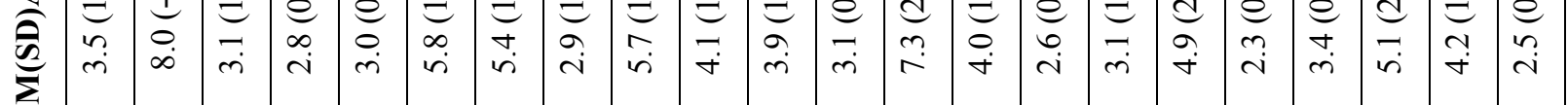

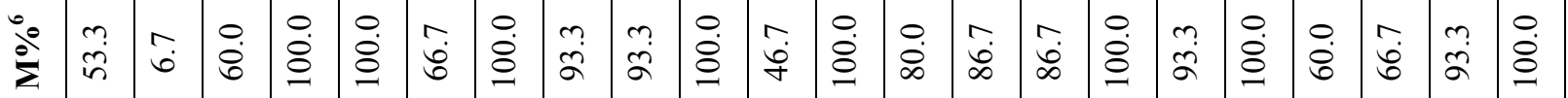

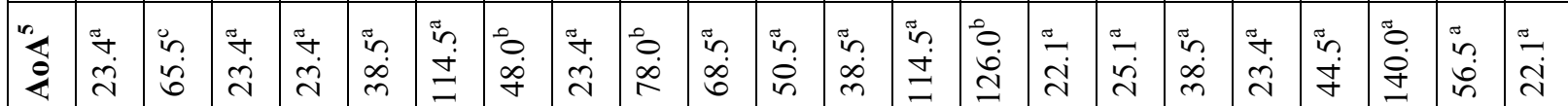

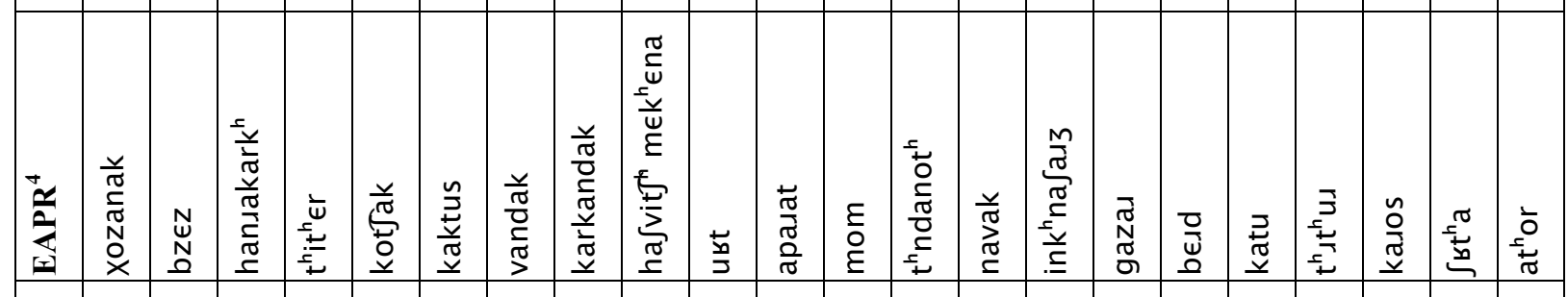

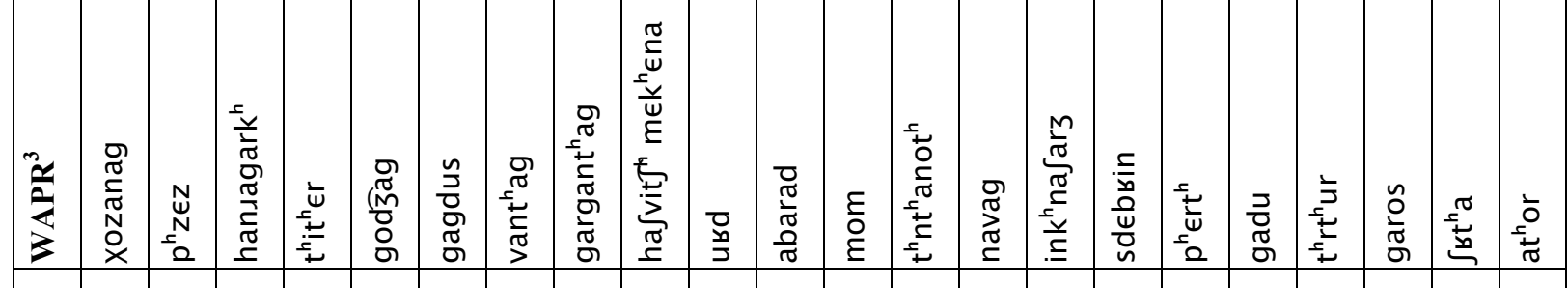

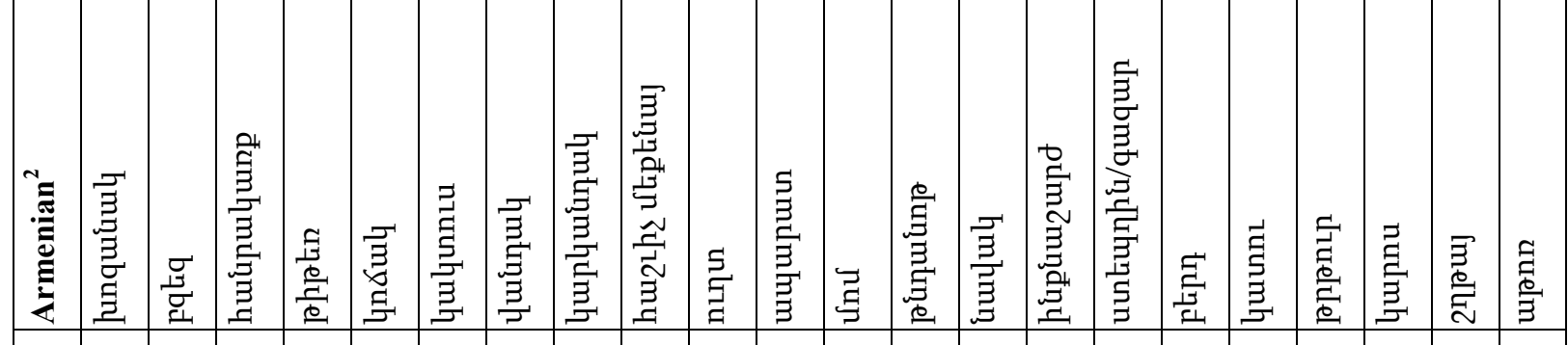

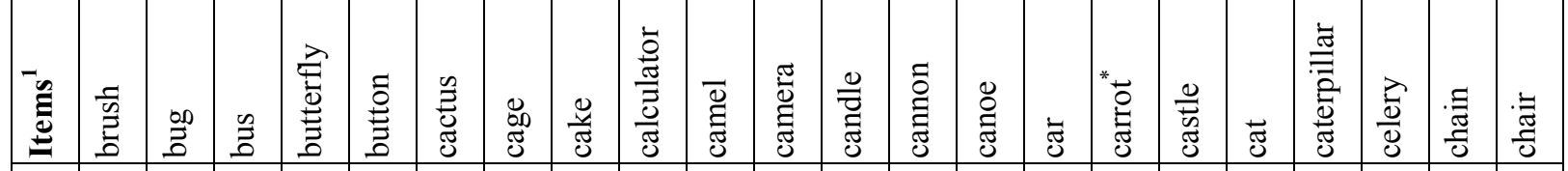

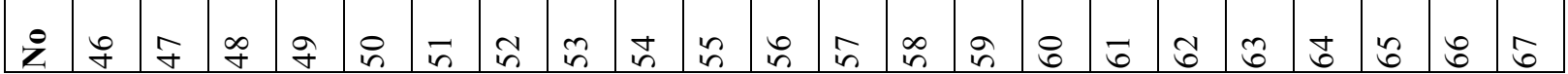




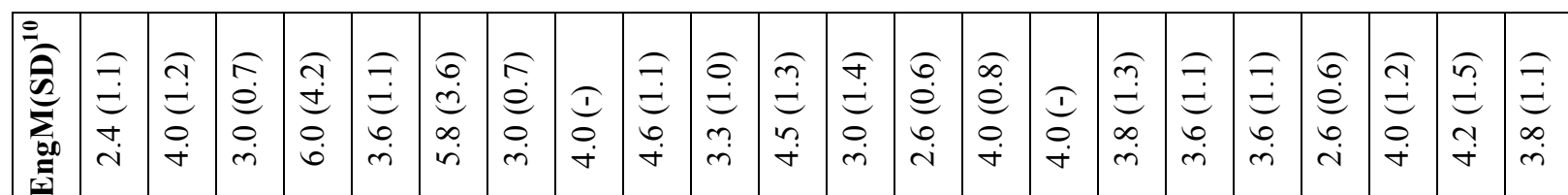

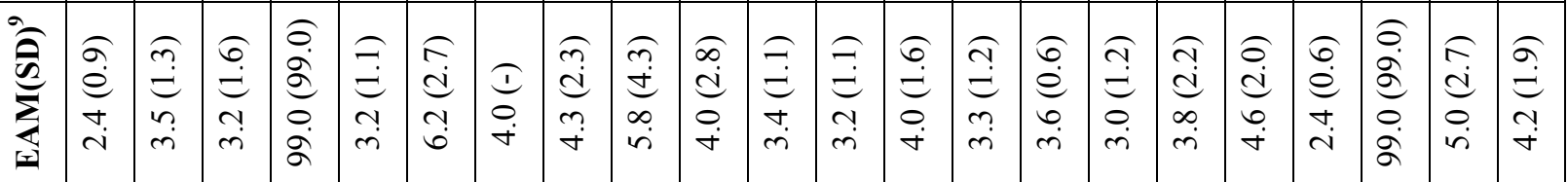

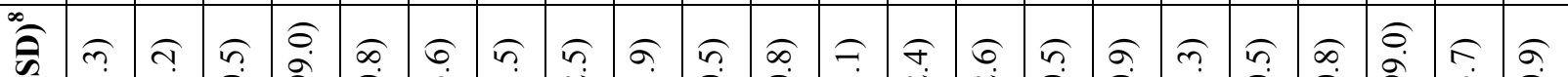

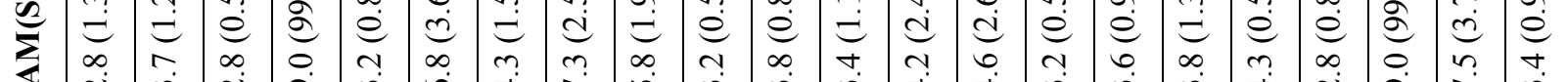

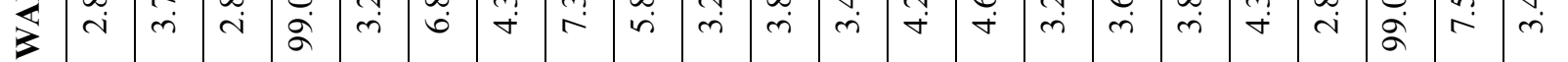

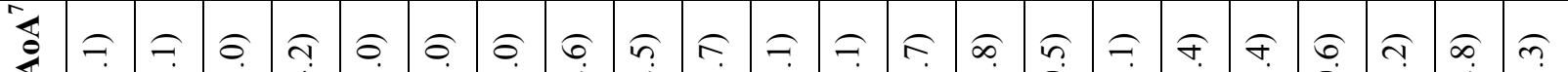

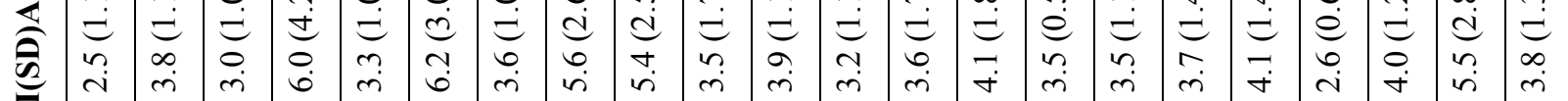

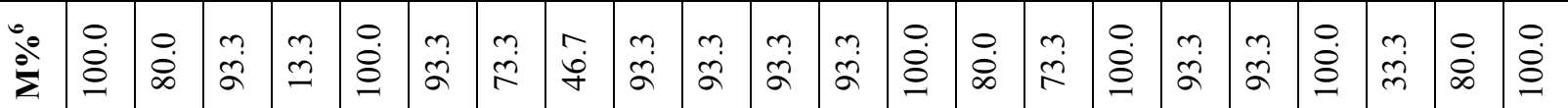

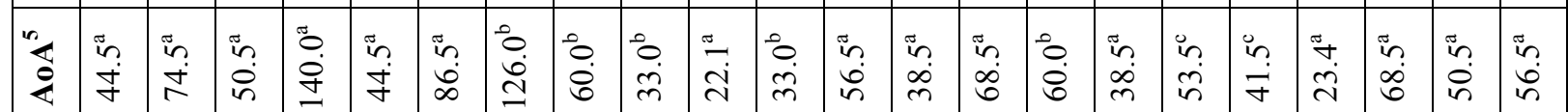

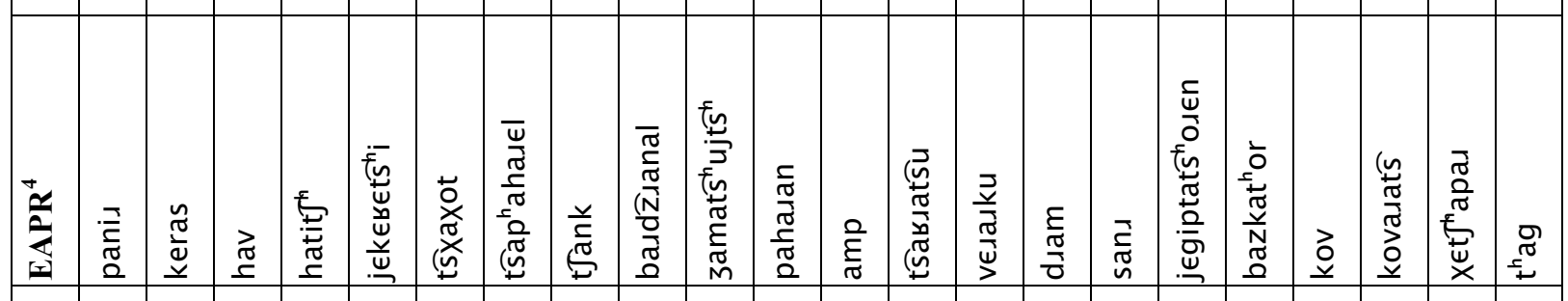

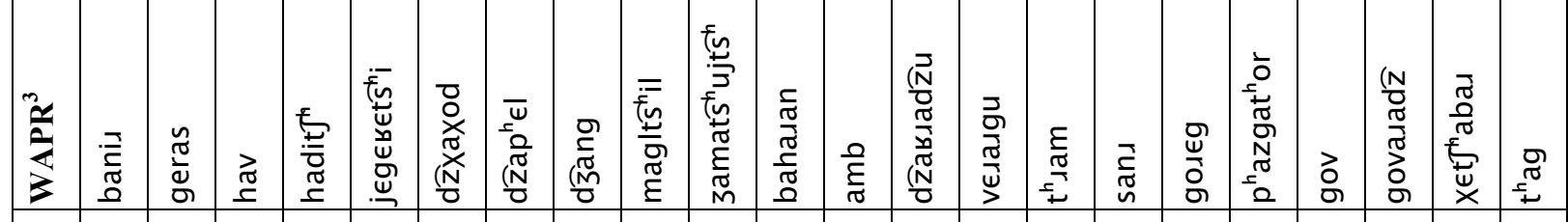

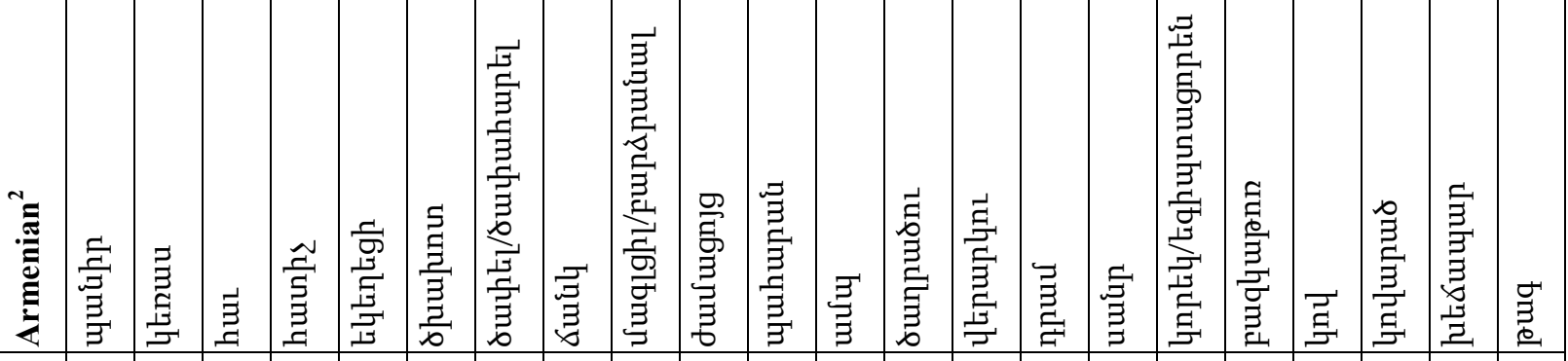

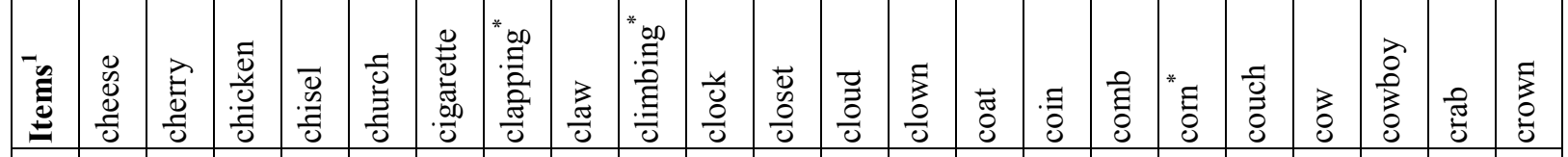

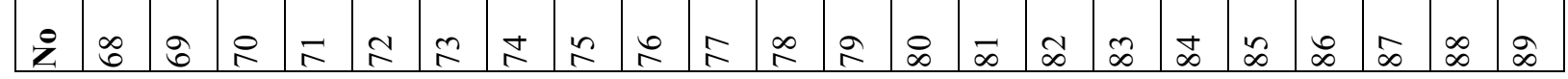




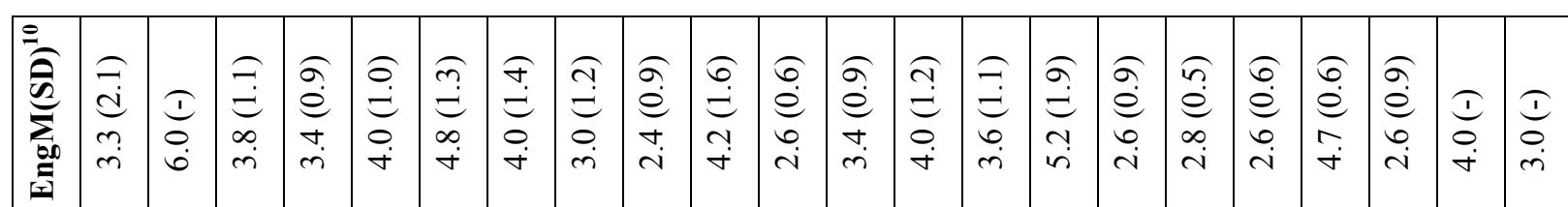

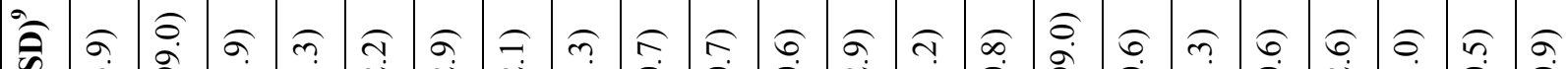

की

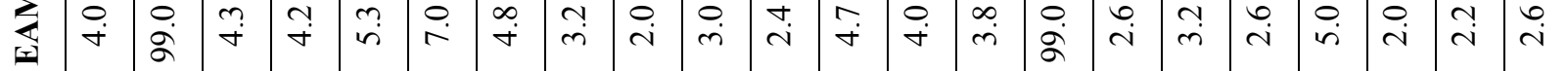

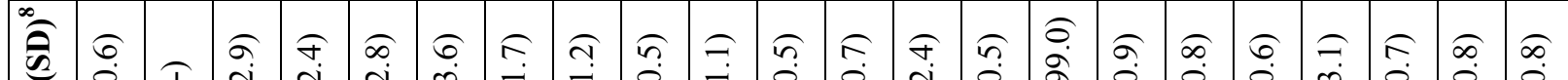

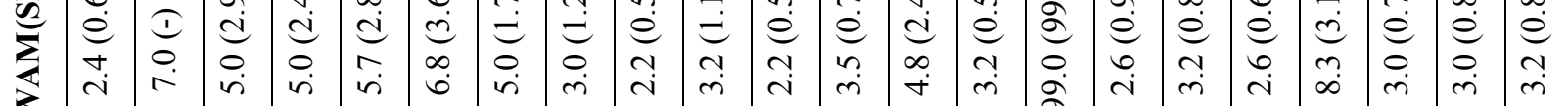

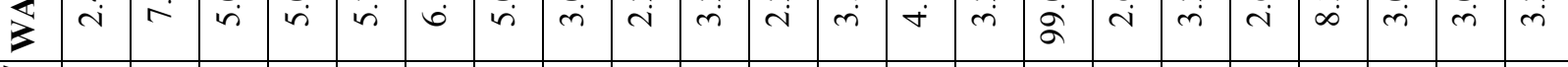

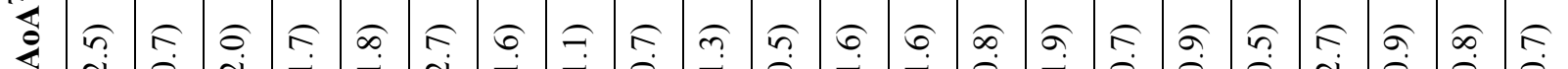

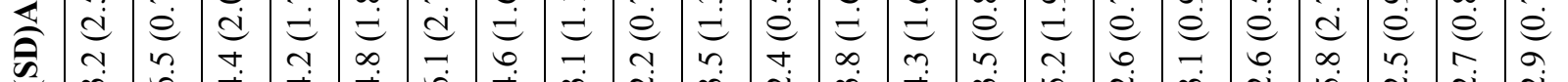

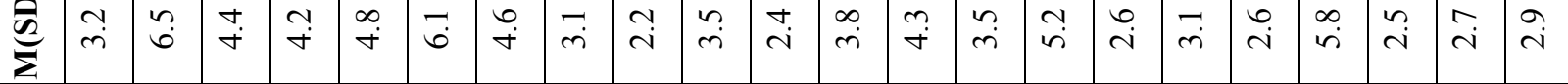

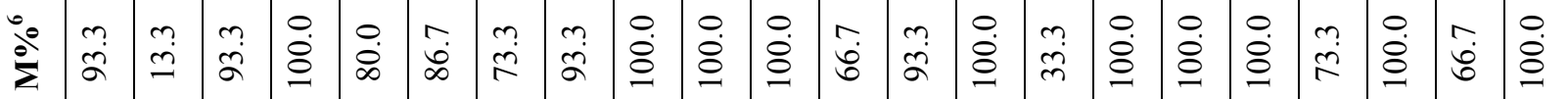

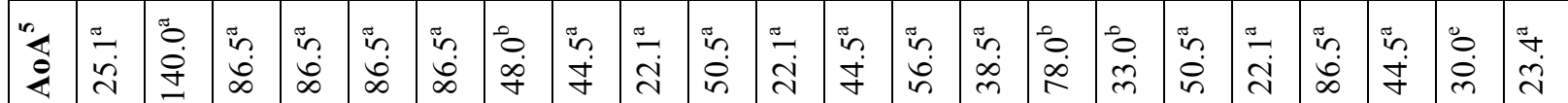

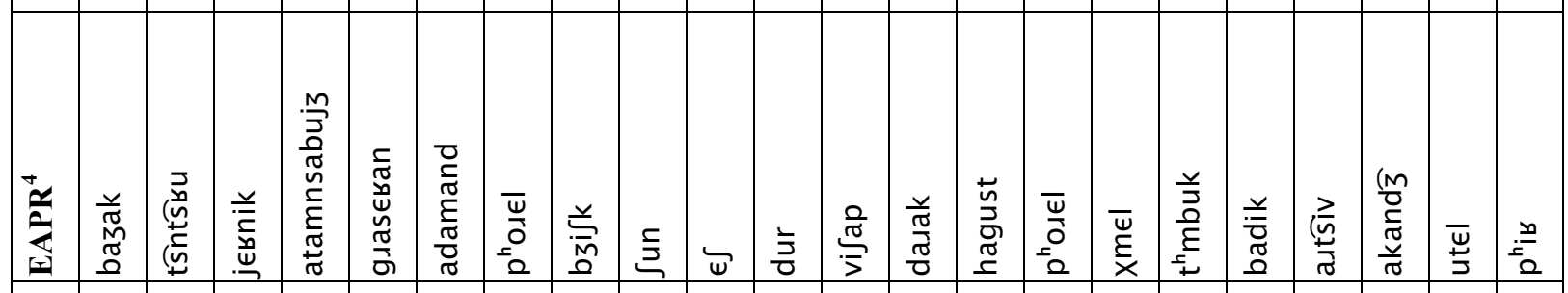

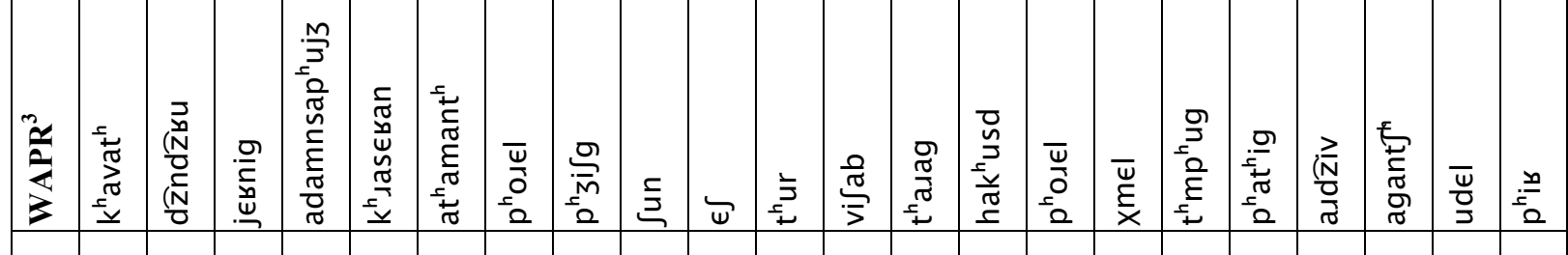

㻤

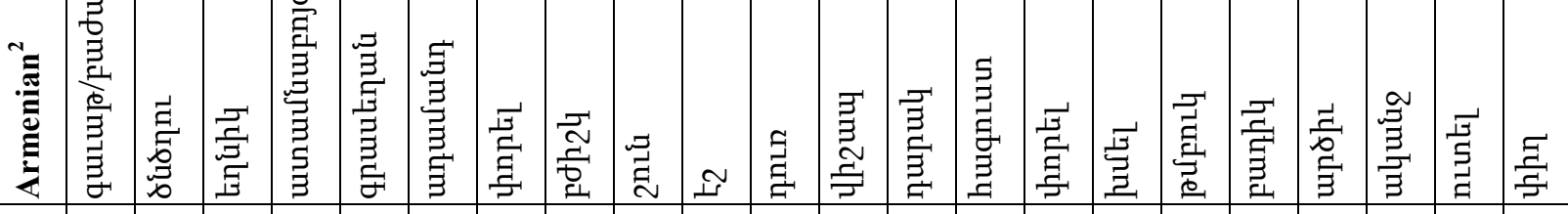

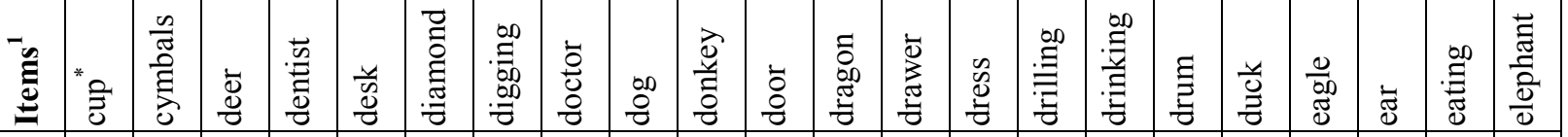

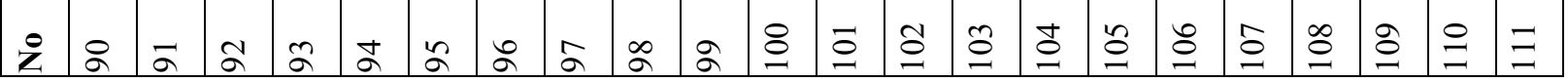




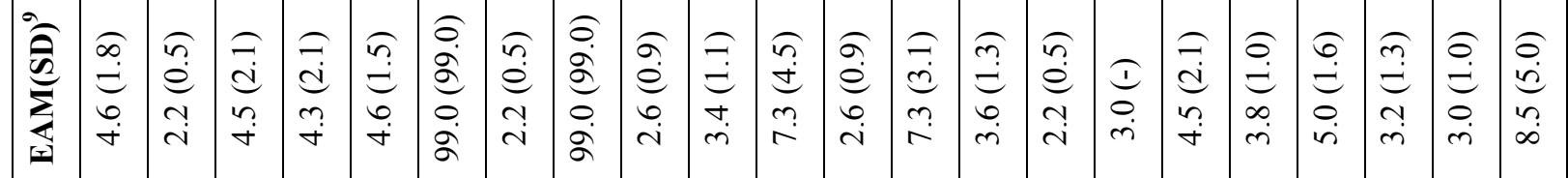

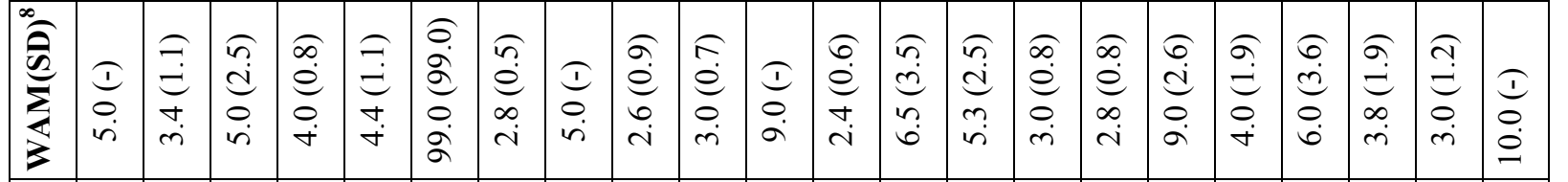

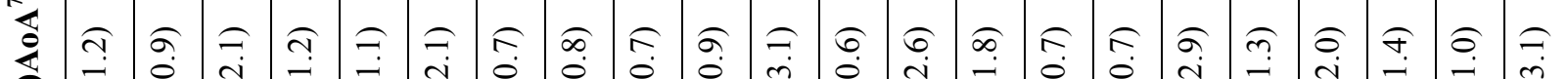

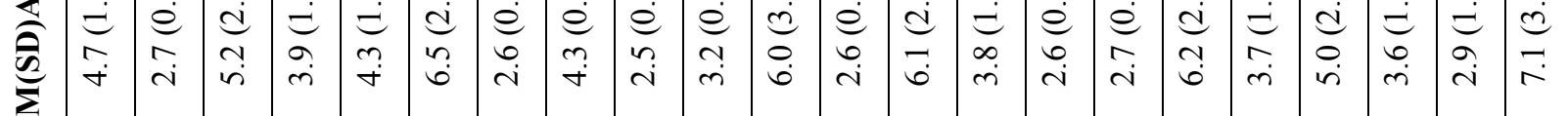

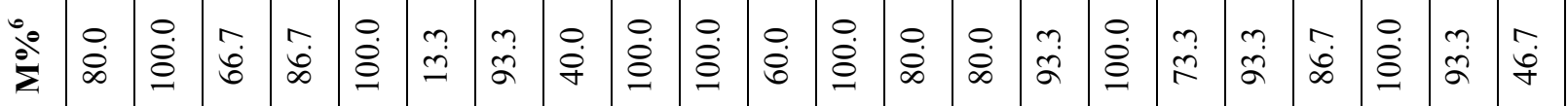

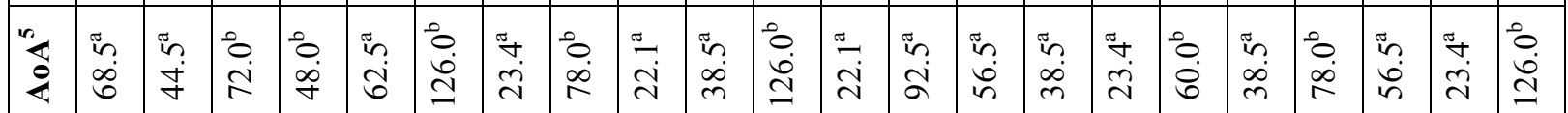

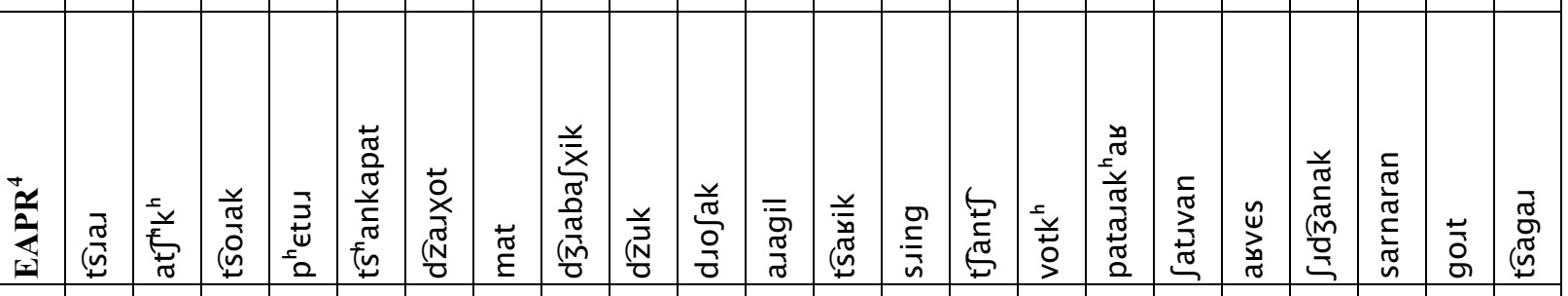

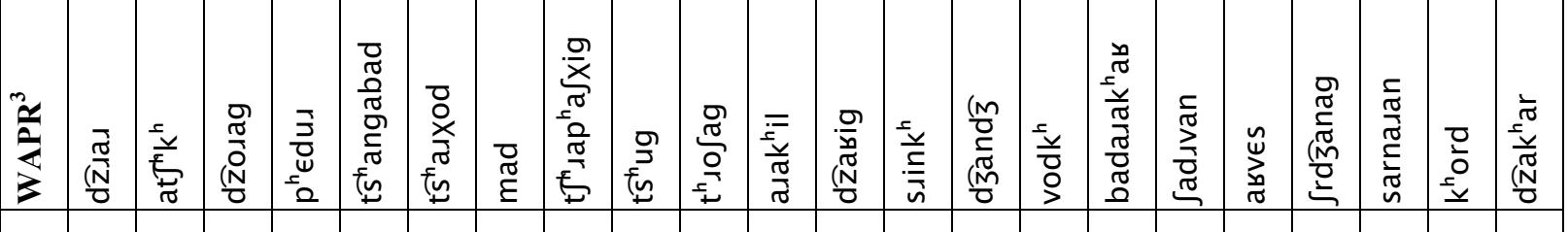

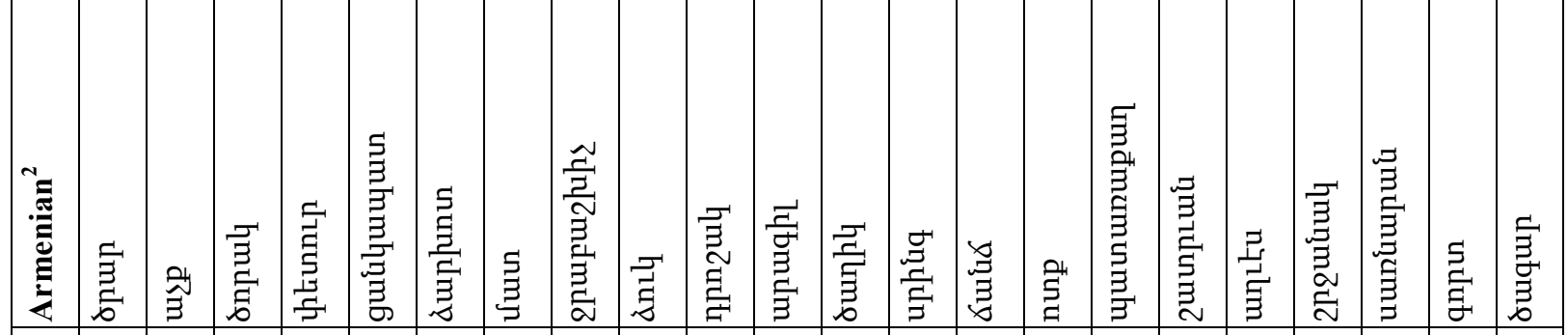

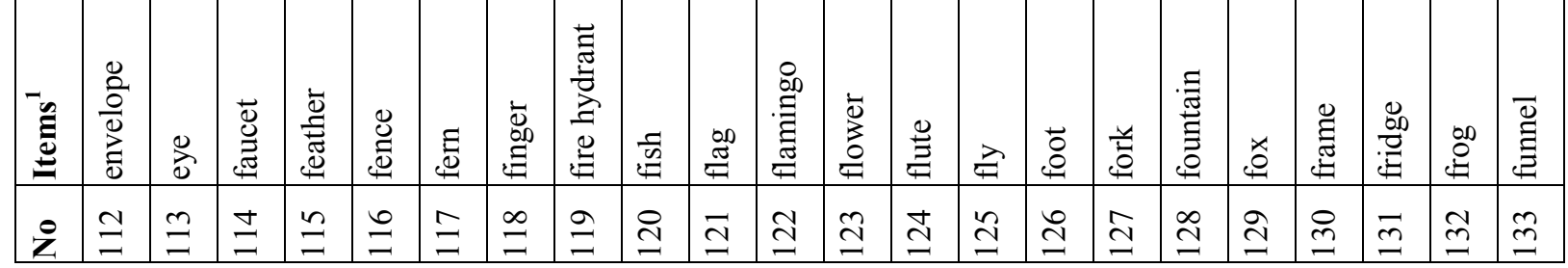




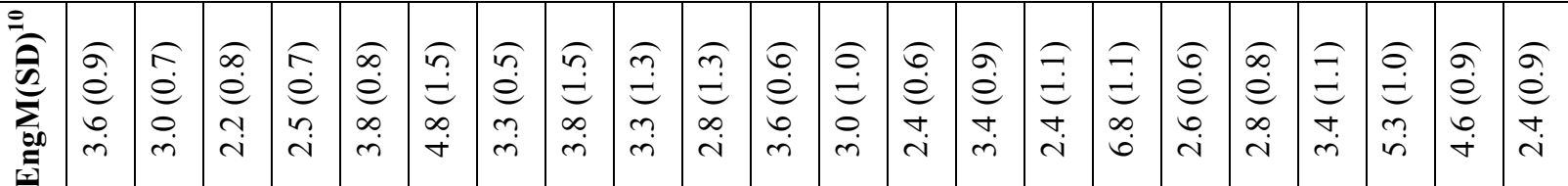

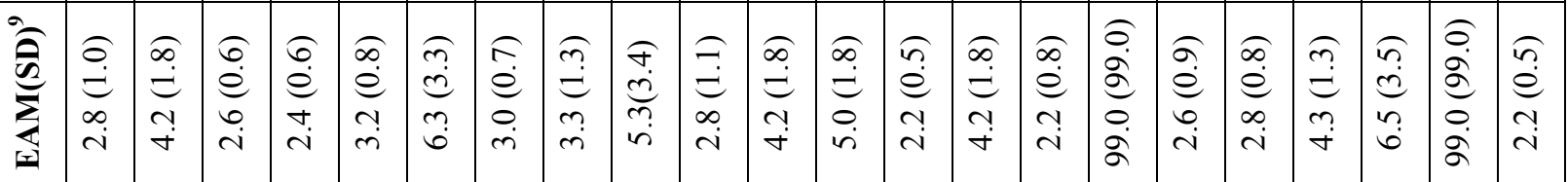

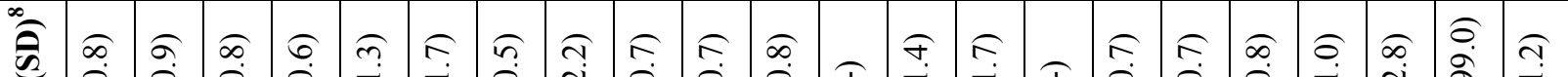

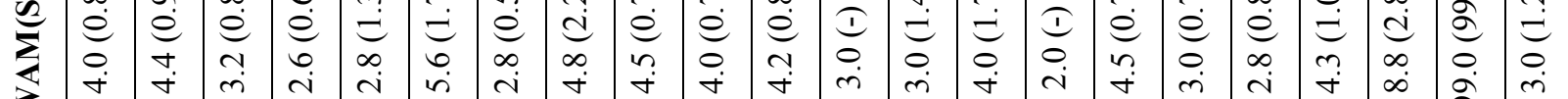

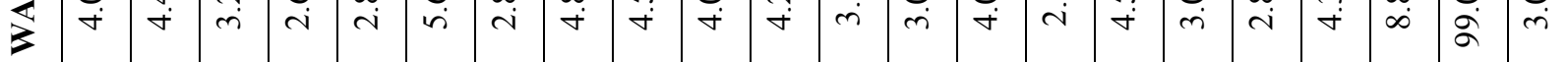

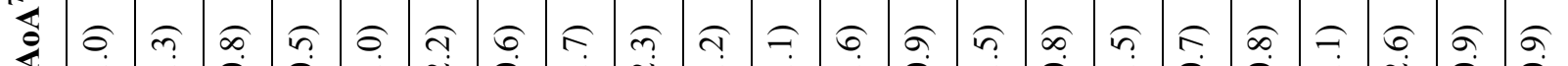

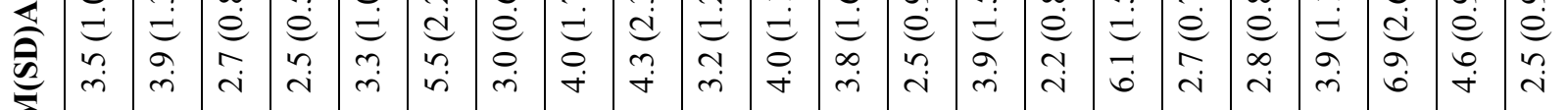

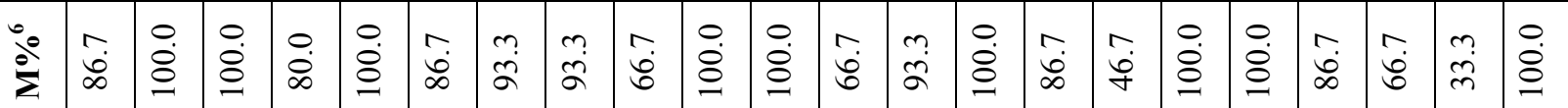

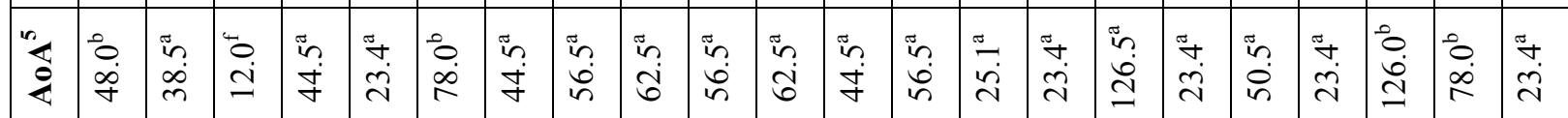

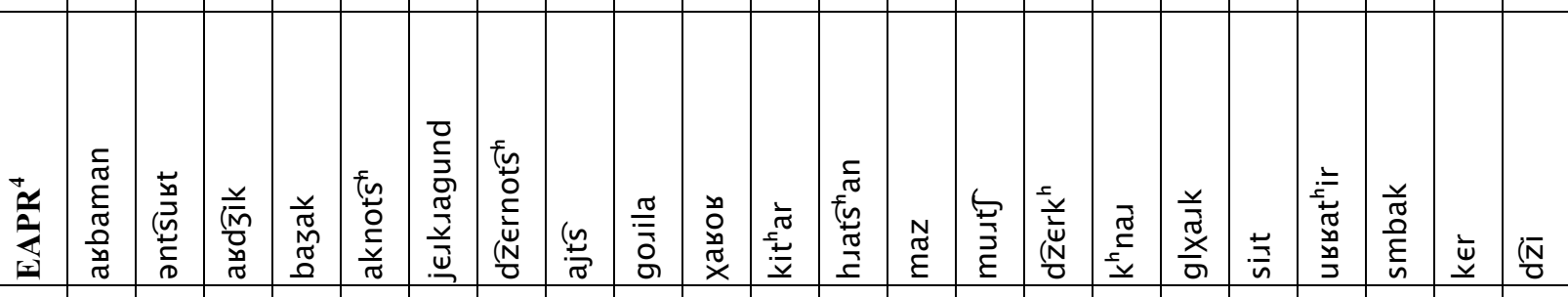

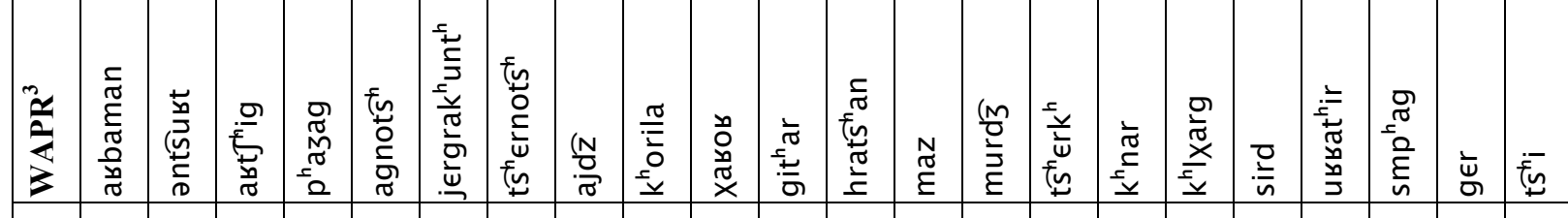

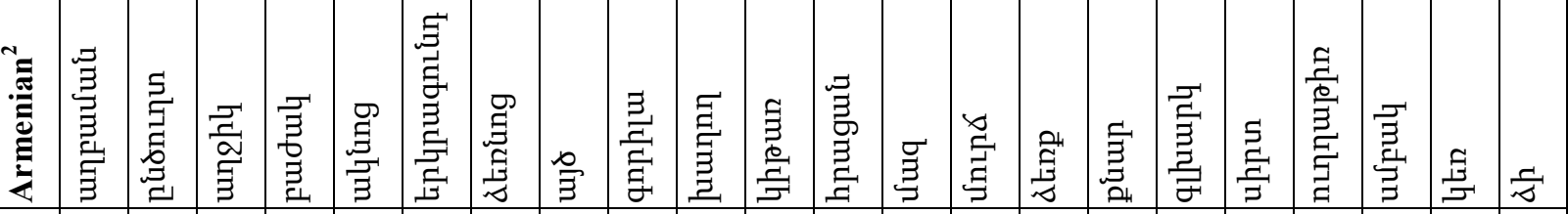

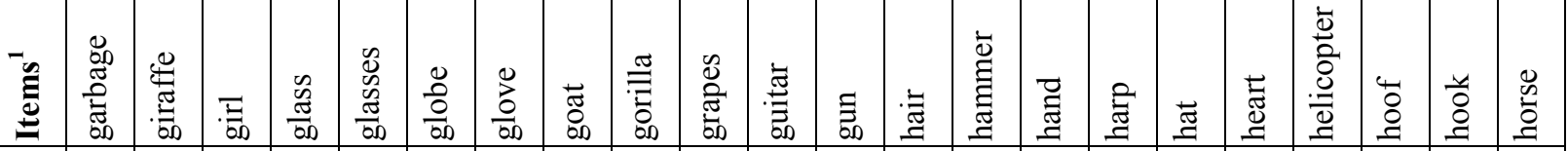

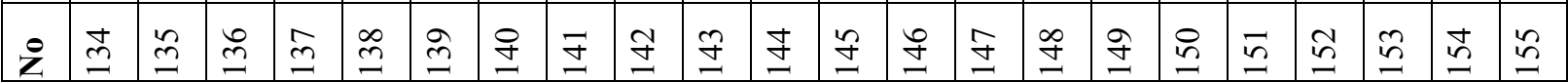




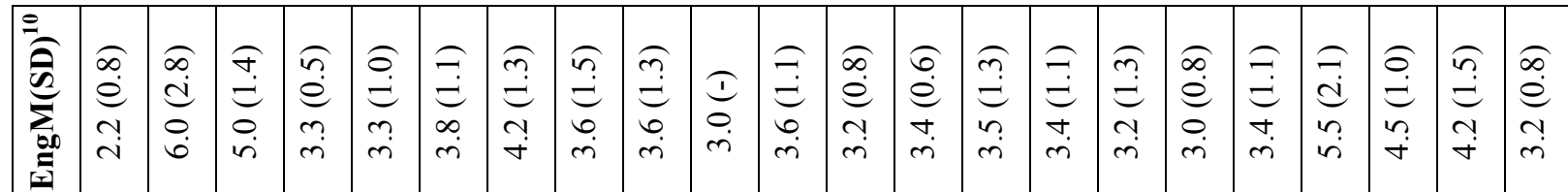

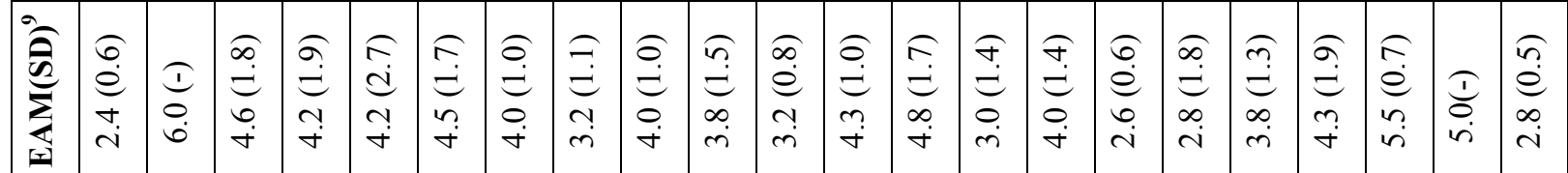

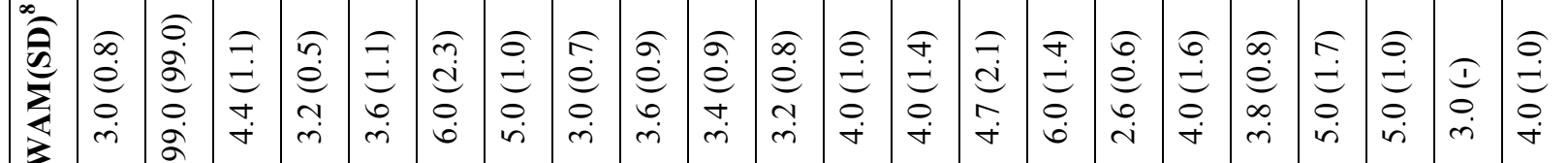

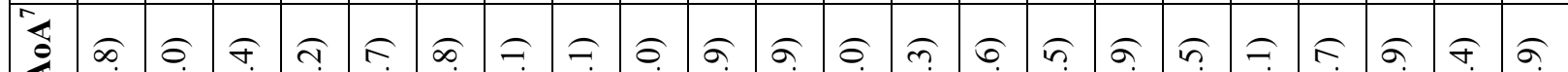

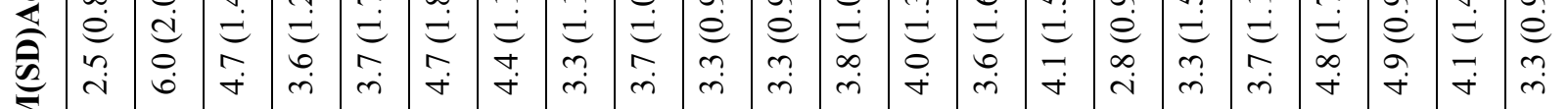

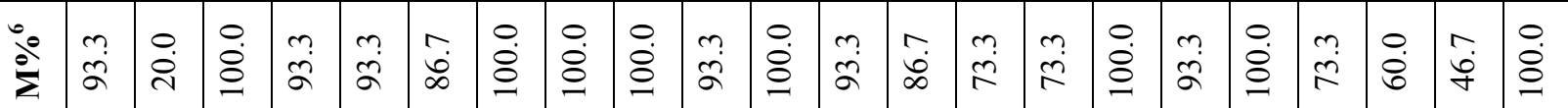

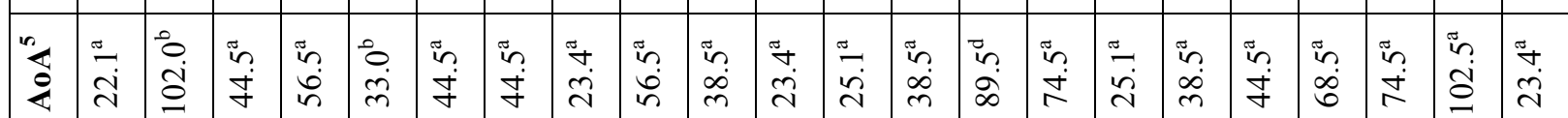

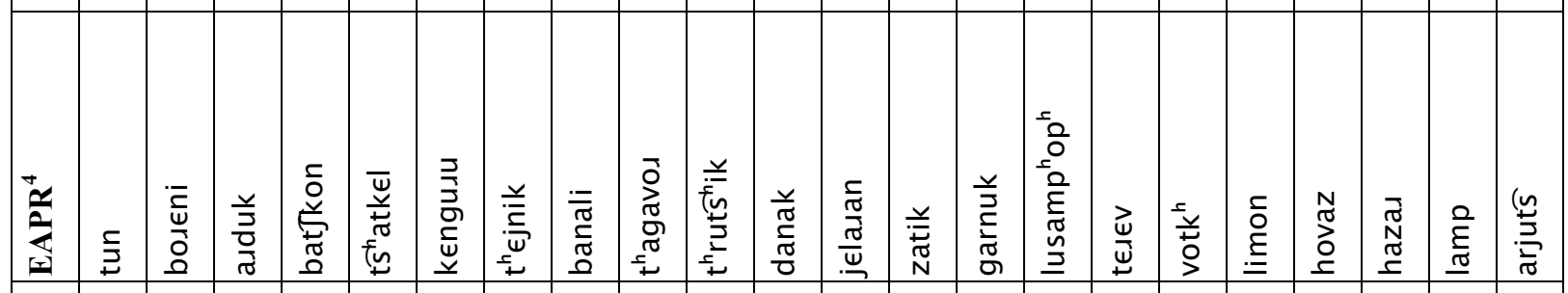

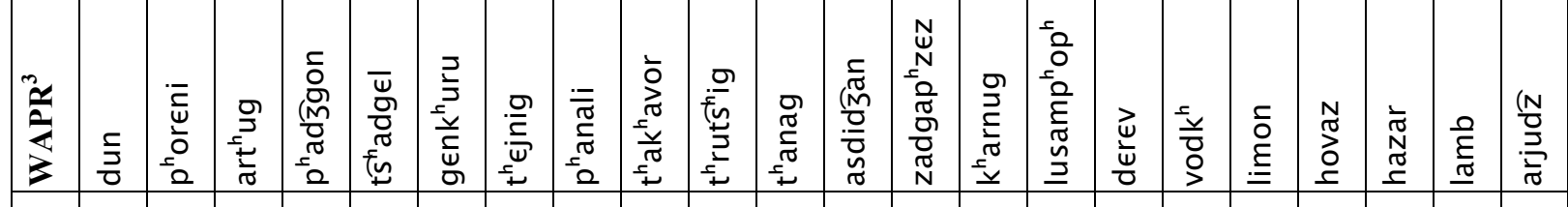

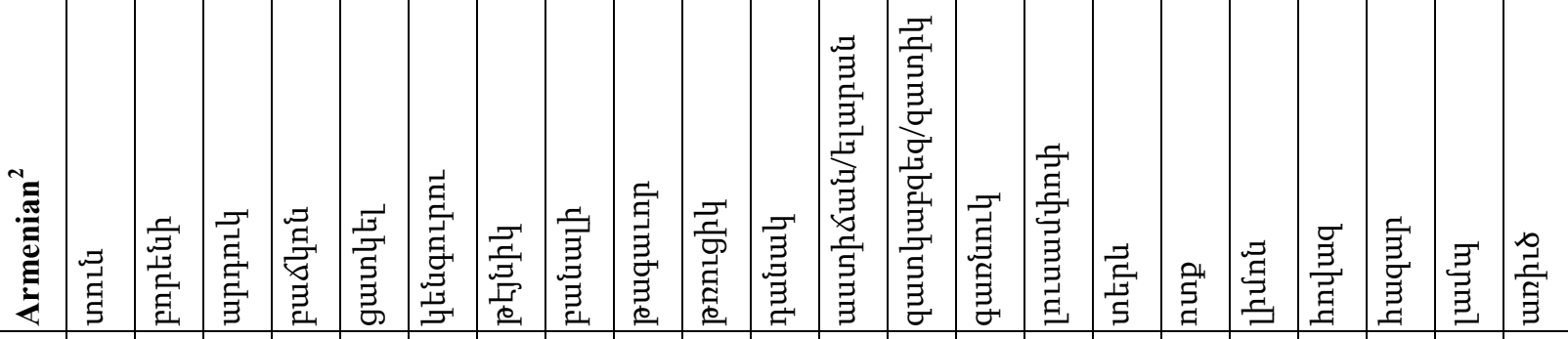

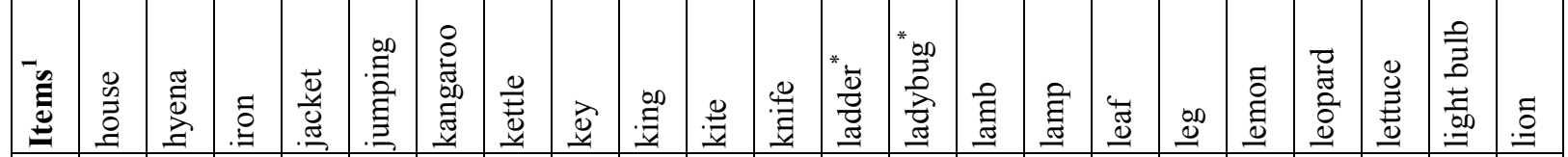

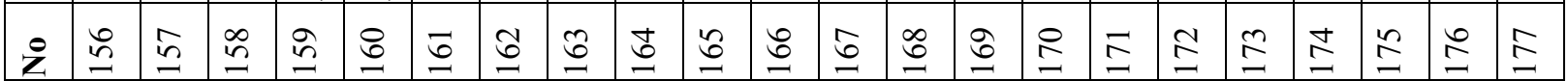




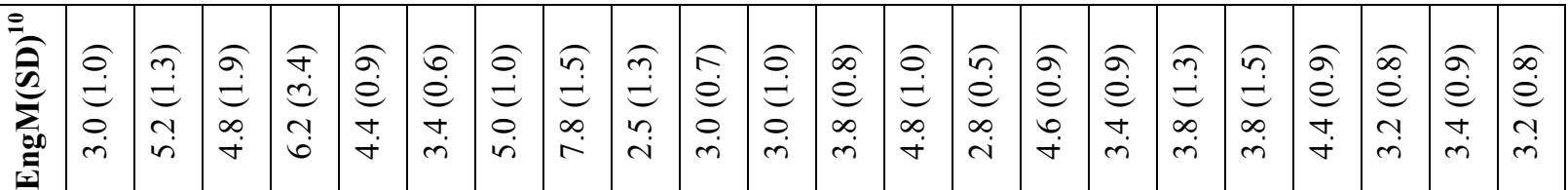

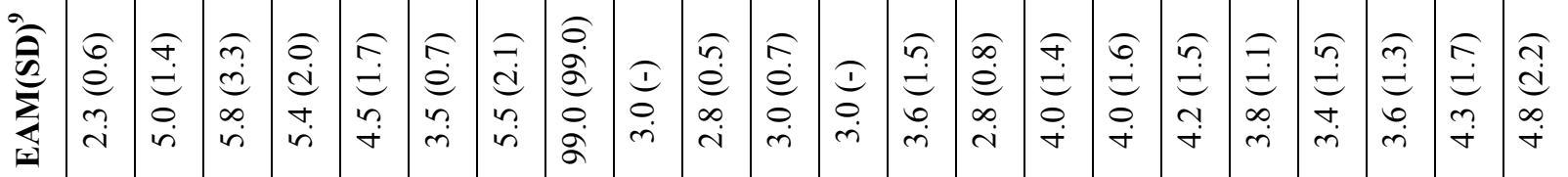

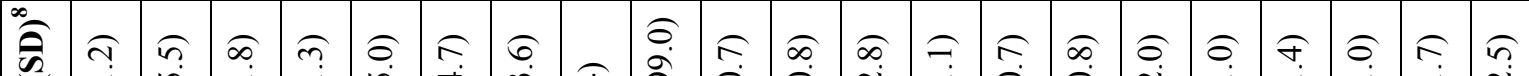

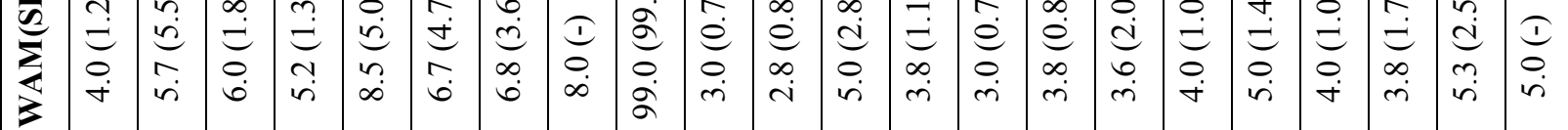

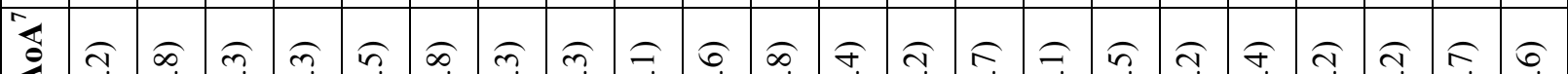

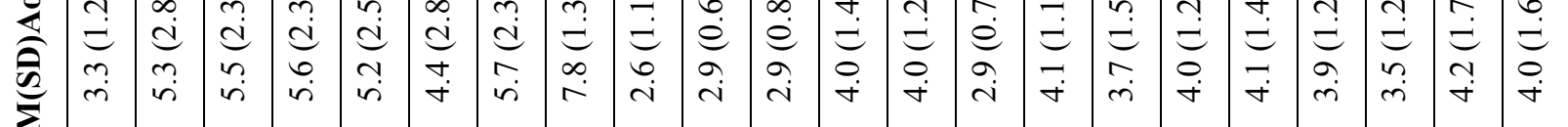

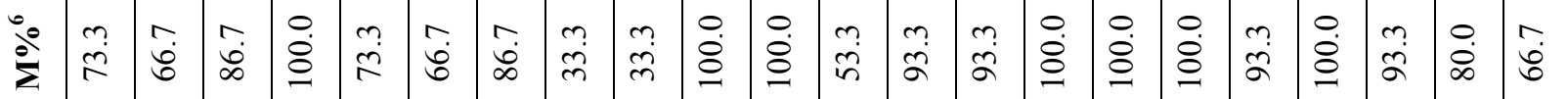

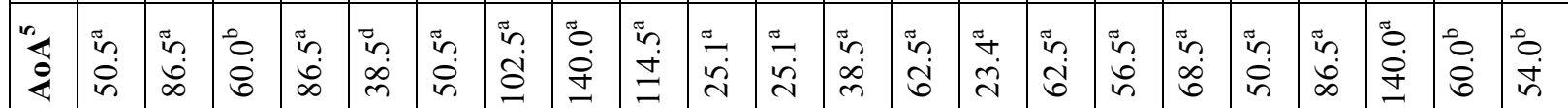

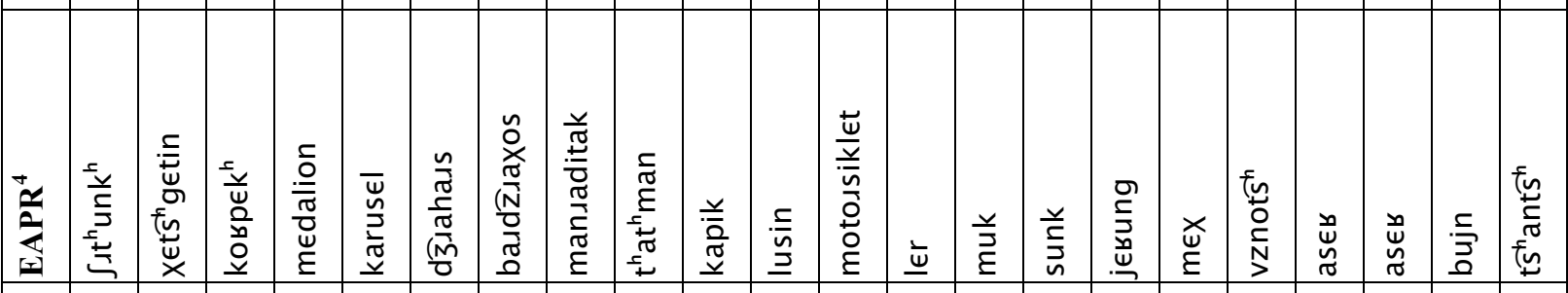

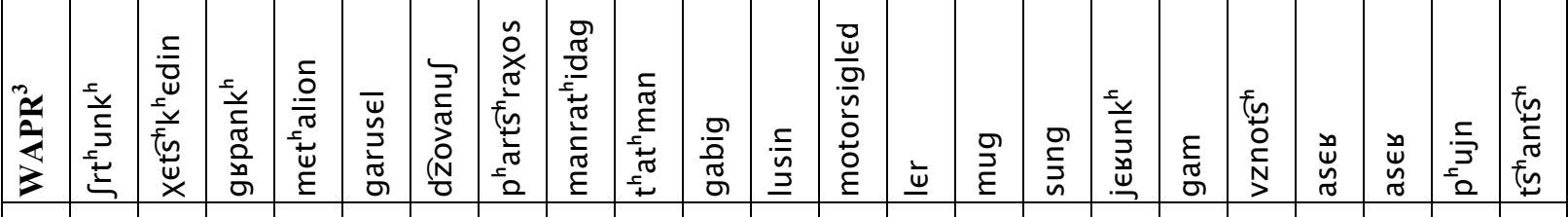

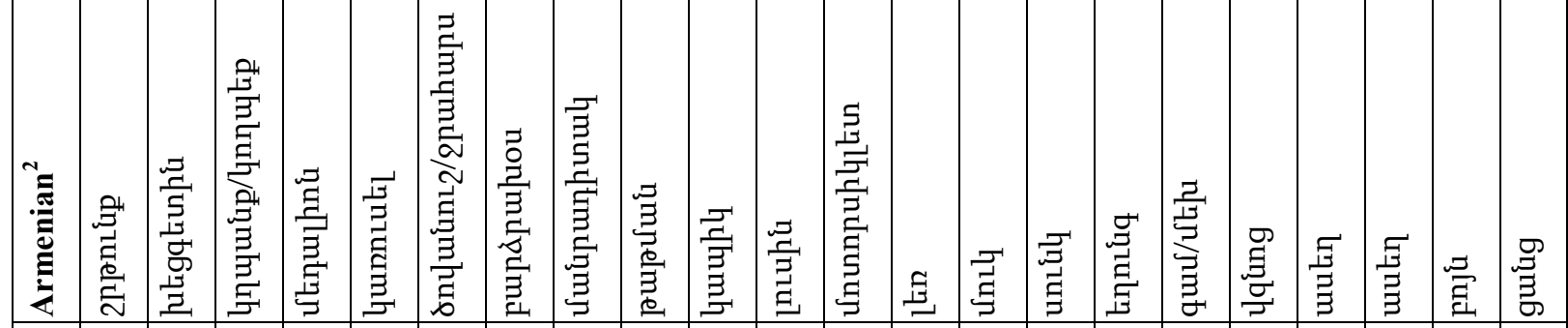

\begin{tabular}{|c|c|c|c|c|c|c|c|c|c|c|c|c|c|c|c|c|c|c|c|c|c|c|}
\hline 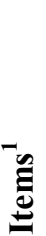 & $\stackrel{\mathscr{2}}{\varrho}$ & $\begin{array}{l}\overrightarrow{0} \\
\stackrel{\tilde{V}}{ } \\
\stackrel{0}{0}\end{array}$ & $\stackrel{*}{*}$ & $\begin{array}{l}\text { ]ే } \\
\text { ¿ }\end{array}$ & 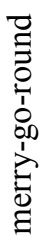 & 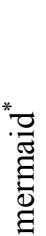 & 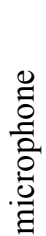 & 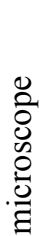 & $\stackrel{\Xi}{\stackrel{\Xi}{\Xi}}$ & $\frac{\grave{d}}{\stackrel{\Xi}{0}}$ & $\begin{array}{l}\text { ठี } \\
\text { ఏ }\end{array}$ & 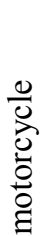 & 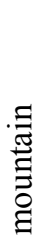 & $\begin{array}{l}\mathscr{E} \\
\Xi \\
\Xi \\
\Xi\end{array}$ & 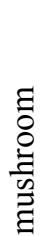 & 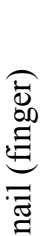 & 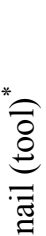 & 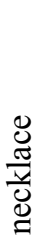 & 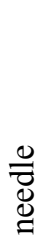 & 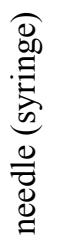 & $\overrightarrow{\mathscr{d}}$ & $\overleftarrow{\Xi}$ \\
\hline$\stackrel{0}{z}$ & $\stackrel{\infty}{\beth}$ & $\stackrel{2}{2}$ & $\stackrel{\infty}{\infty}$ & $\infty$ & $\infty$ & $\infty$ & $\underset{D}{\triangleright}$ & 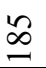 & $\stackrel{\infty}{\infty}$ & $\infty$ & $\stackrel{\infty}{\infty}$ & ळ & $\stackrel{\circ}{\circ}$ & $\bar{a}$ & ๙ & 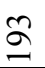 & よ & $\stackrel{n}{2}$ & 음 & $\hat{a}$ & $\stackrel{\infty}{\Omega}$ & $\sigma$ \\
\hline
\end{tabular}


$\underline{6}$

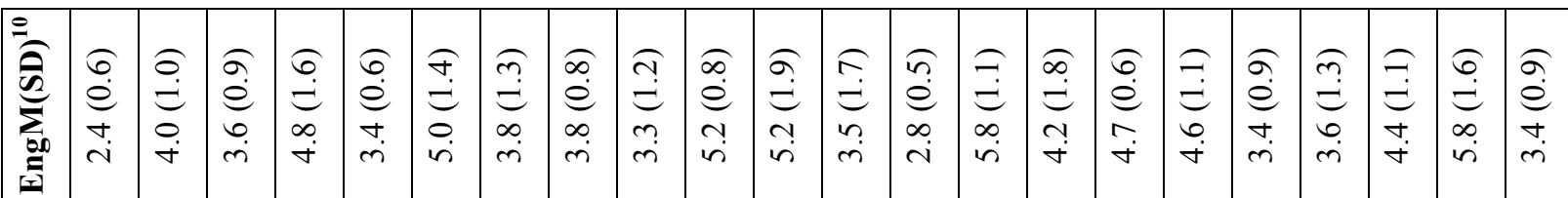

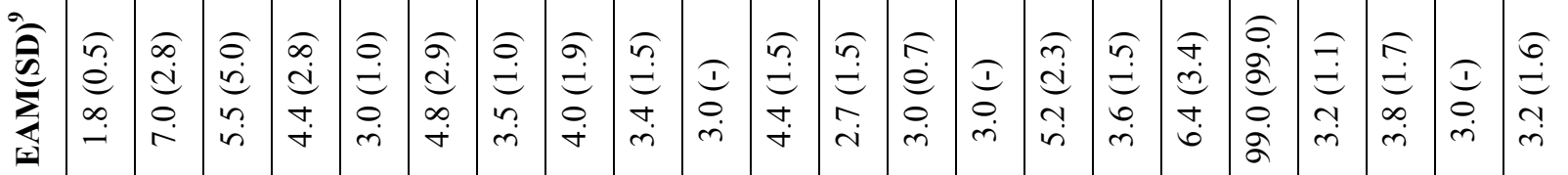

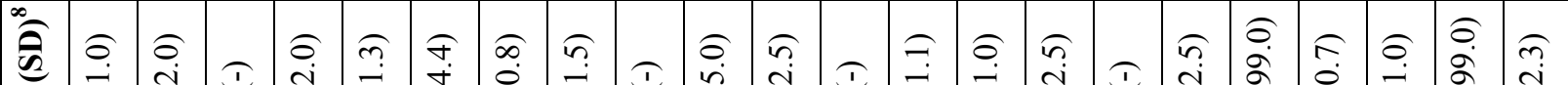

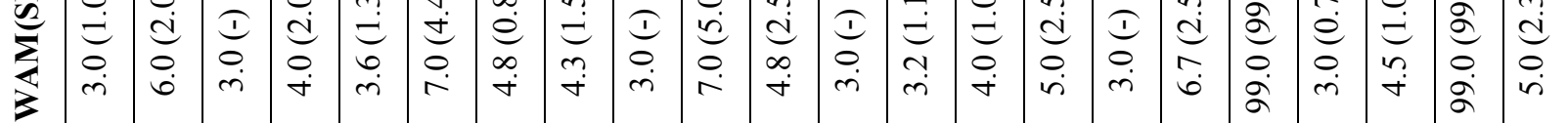

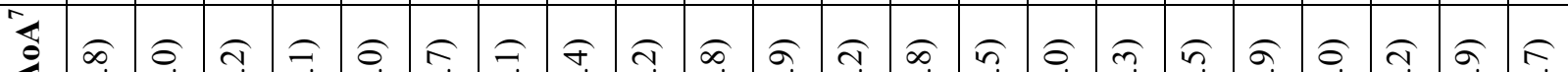

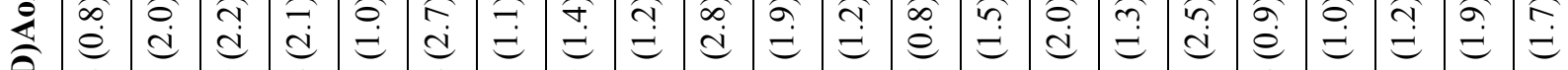

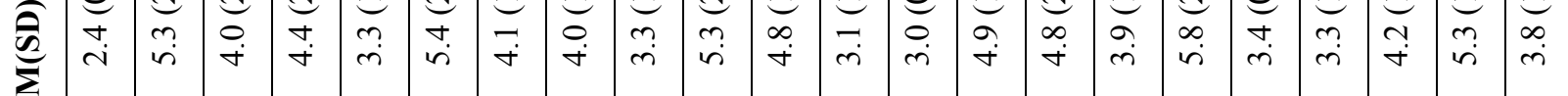

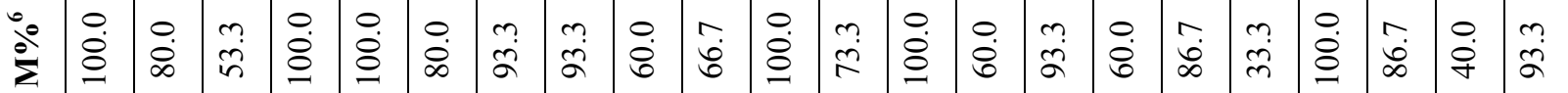

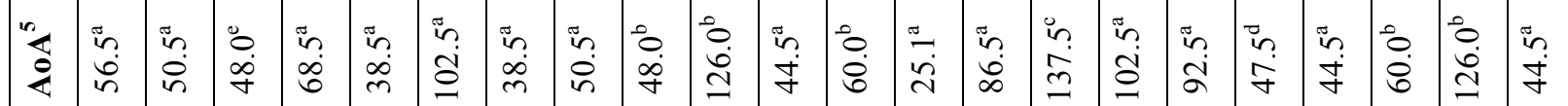

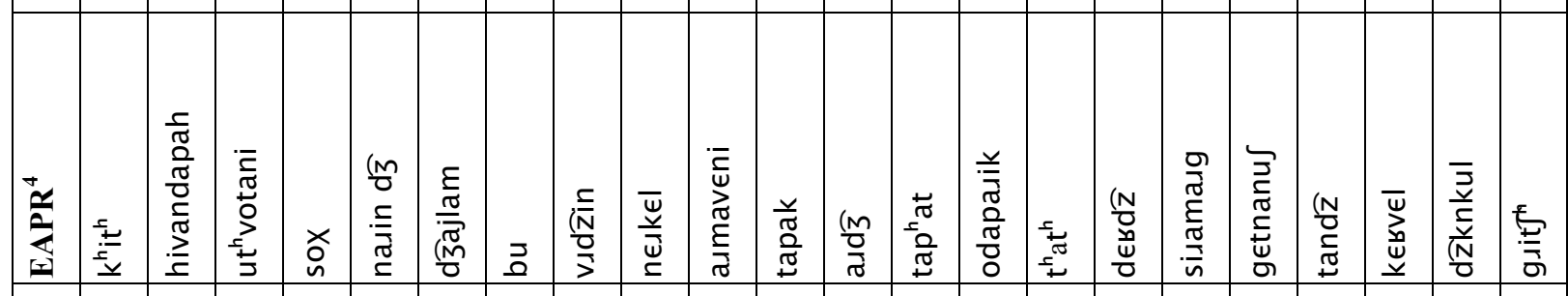

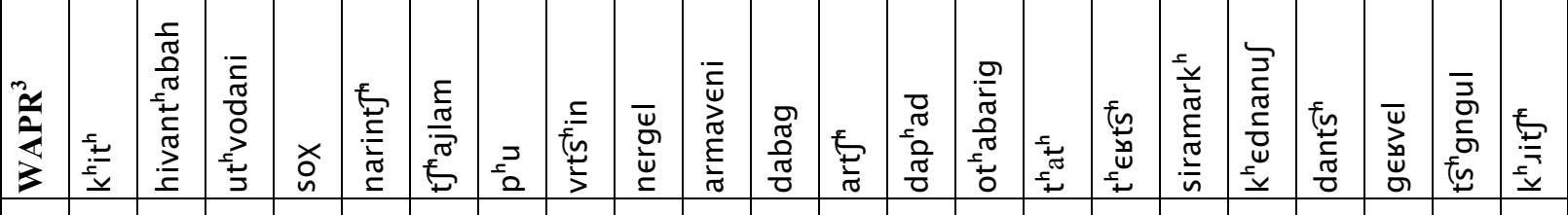

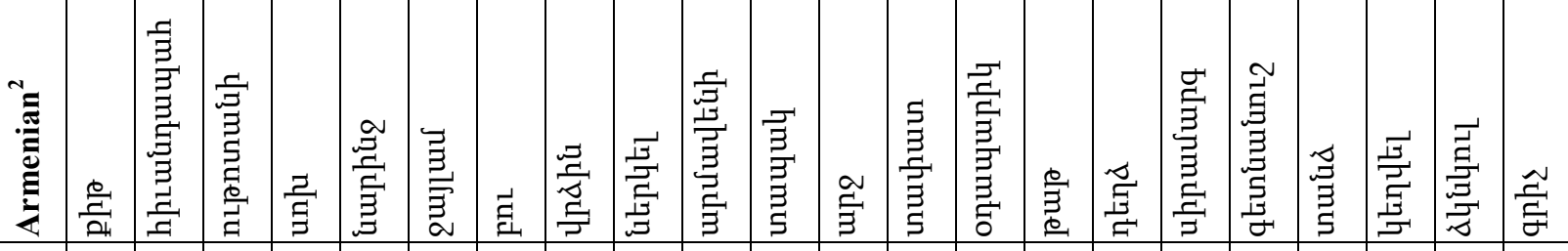

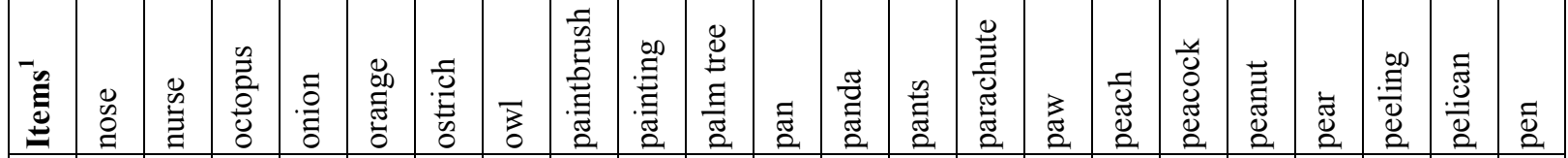

そ 


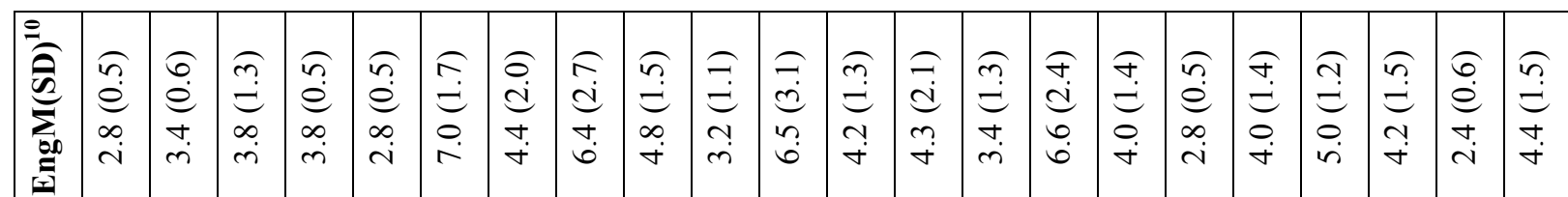

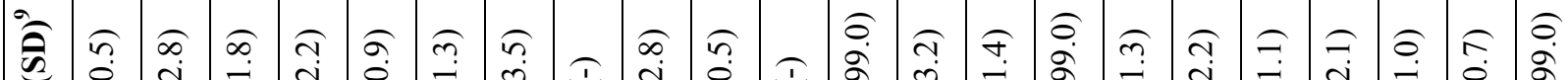

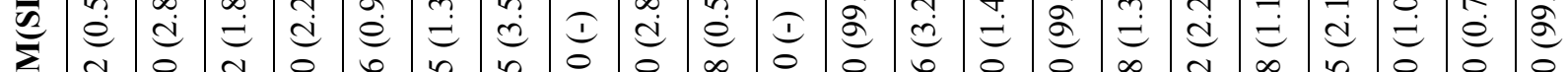

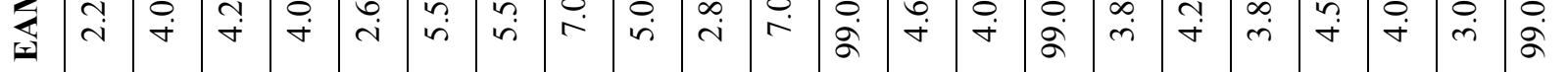

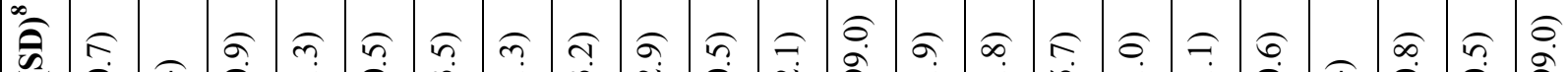

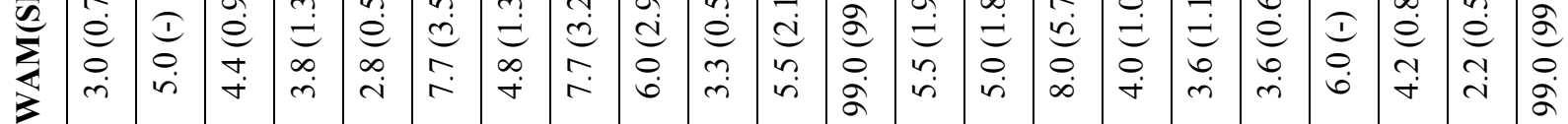

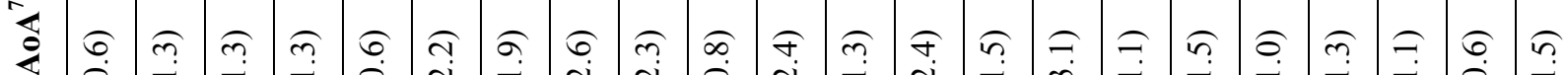

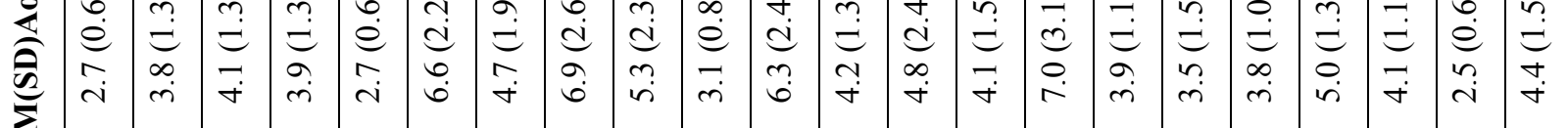

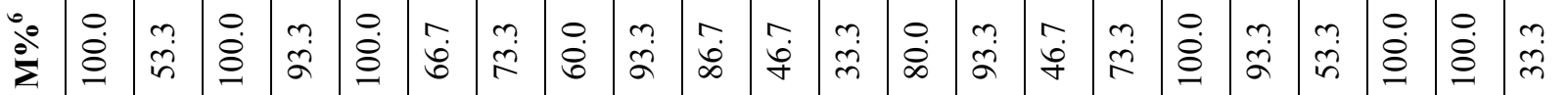

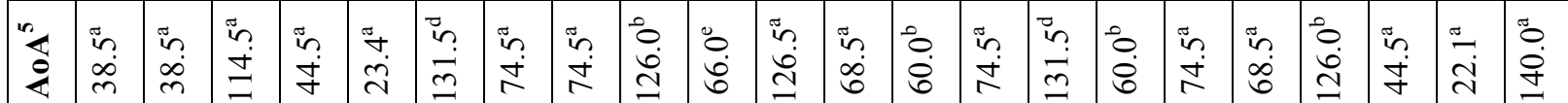

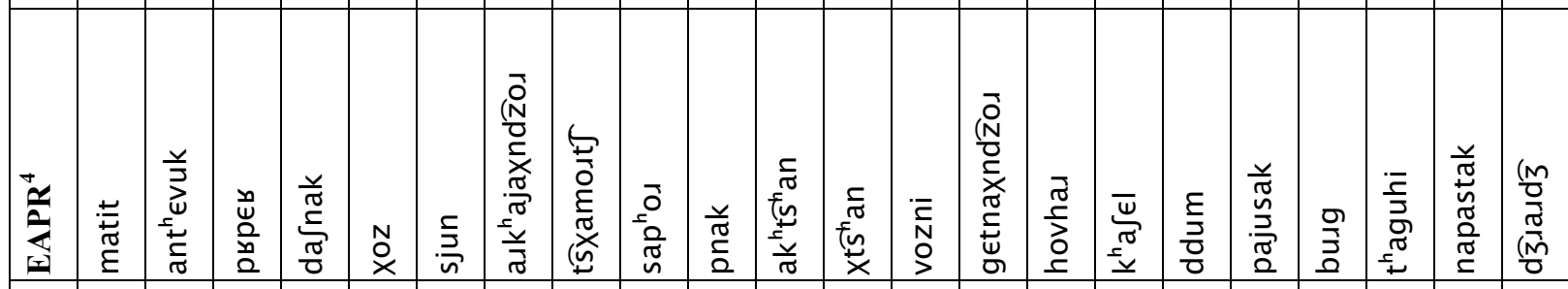

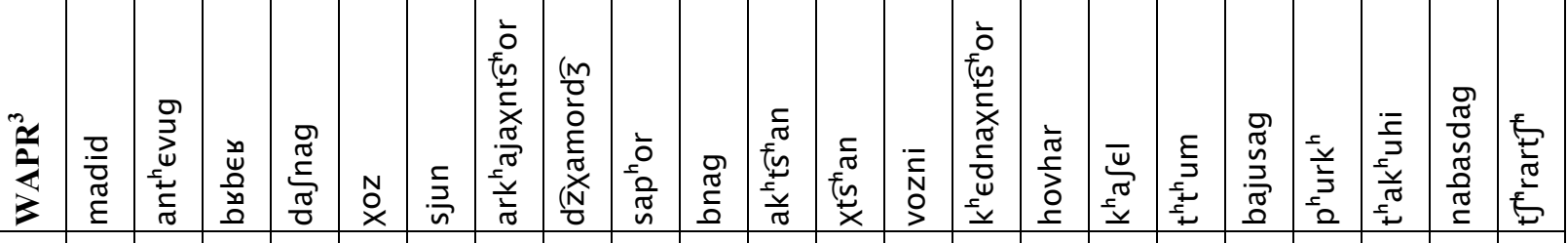

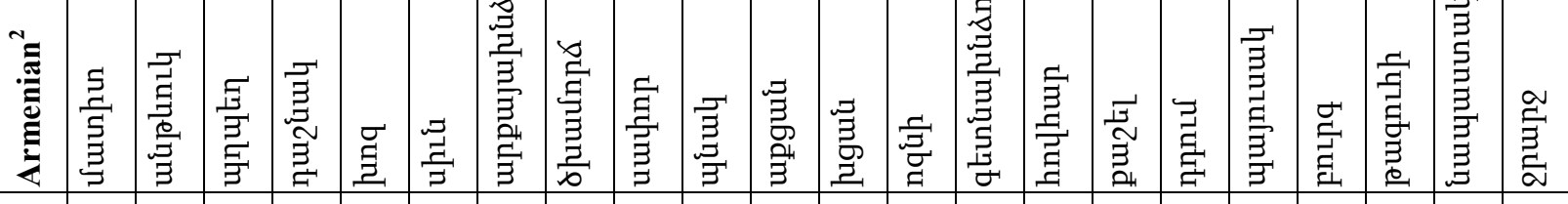

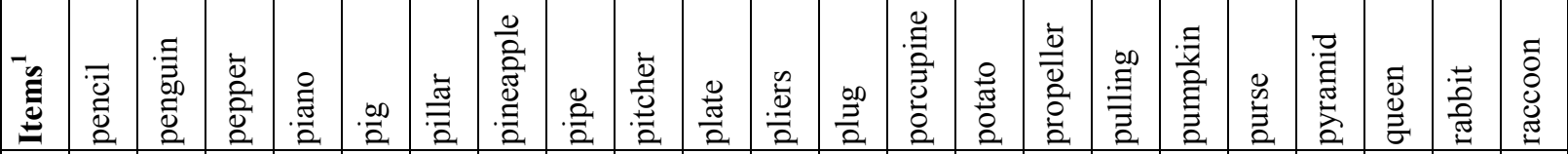

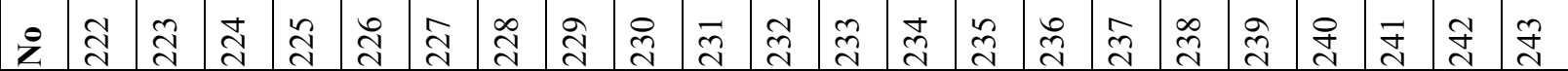


$\widehat{6}$

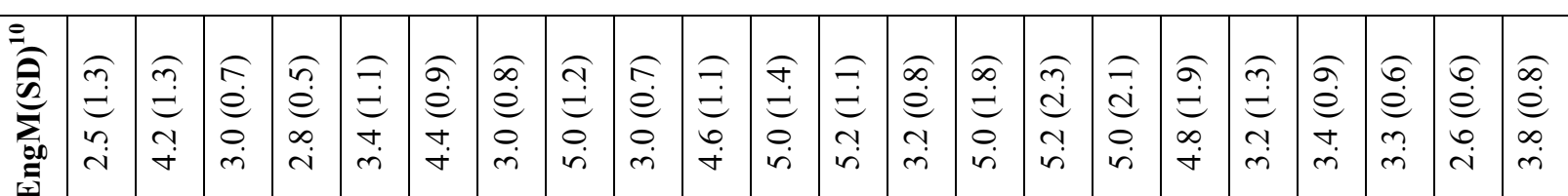

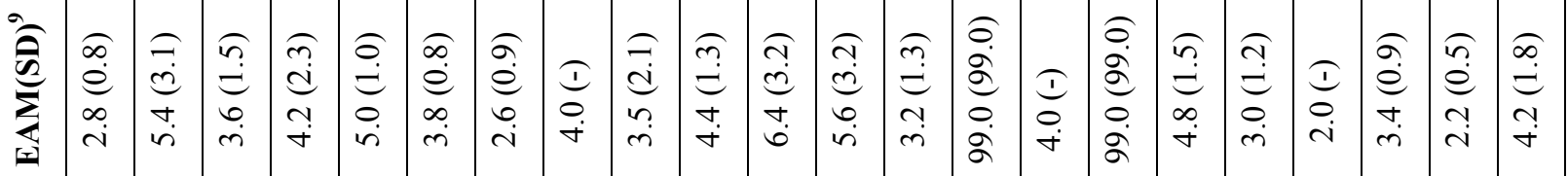

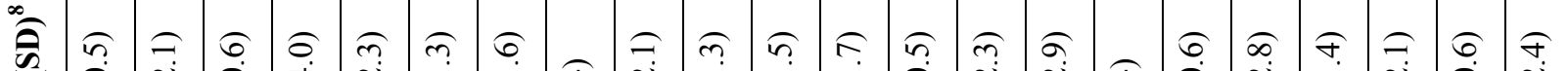

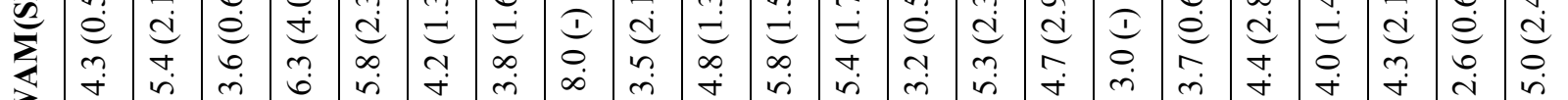

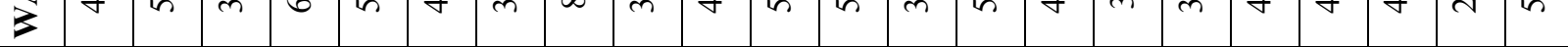

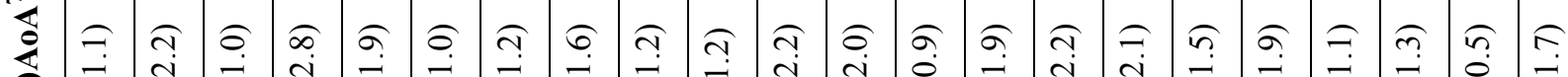

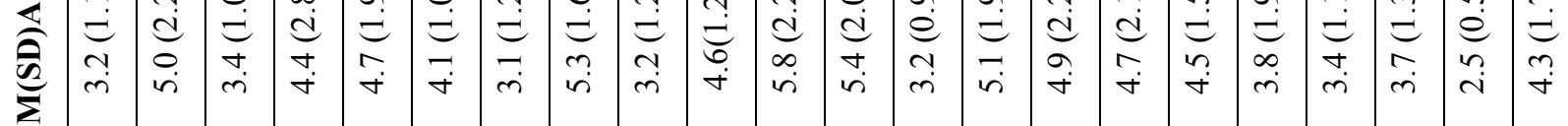

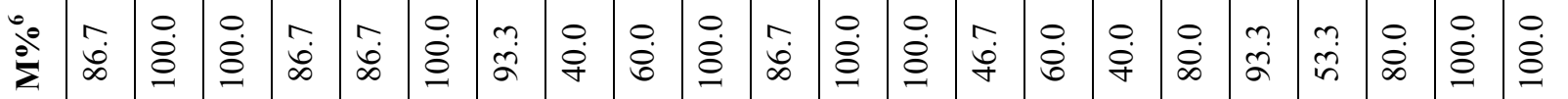

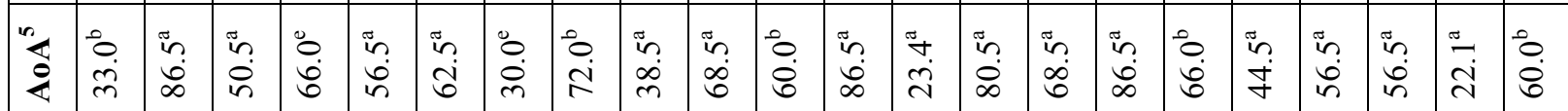

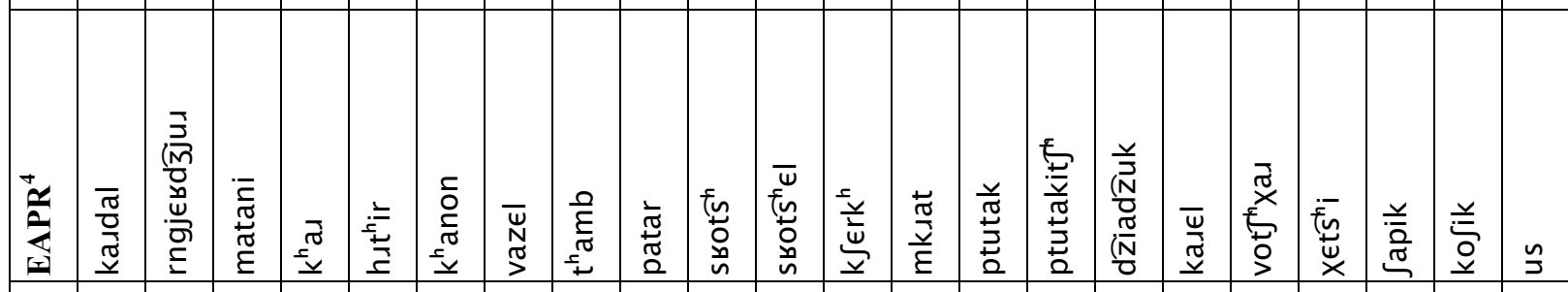

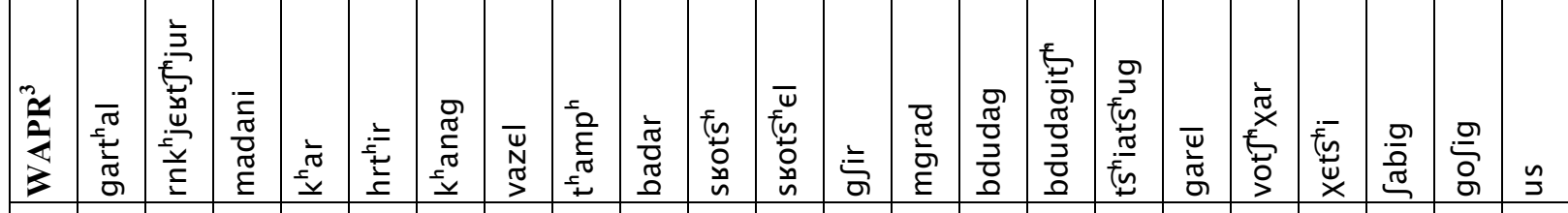

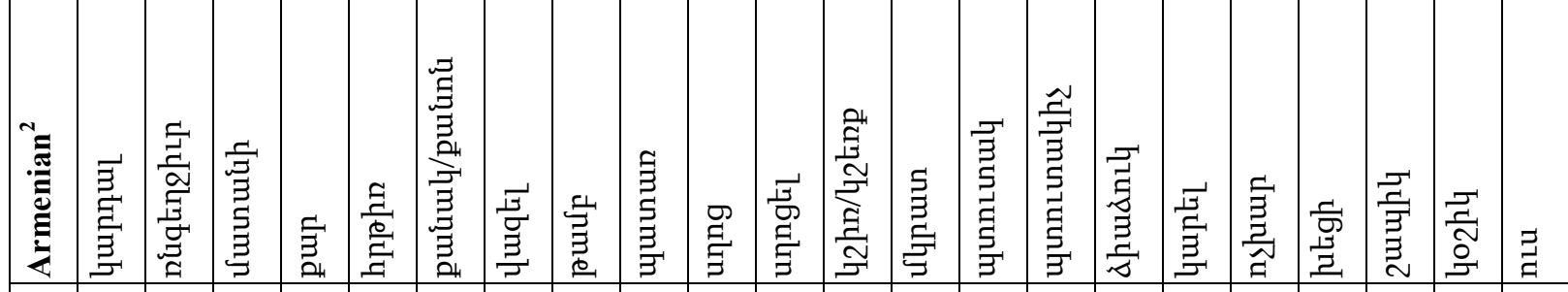

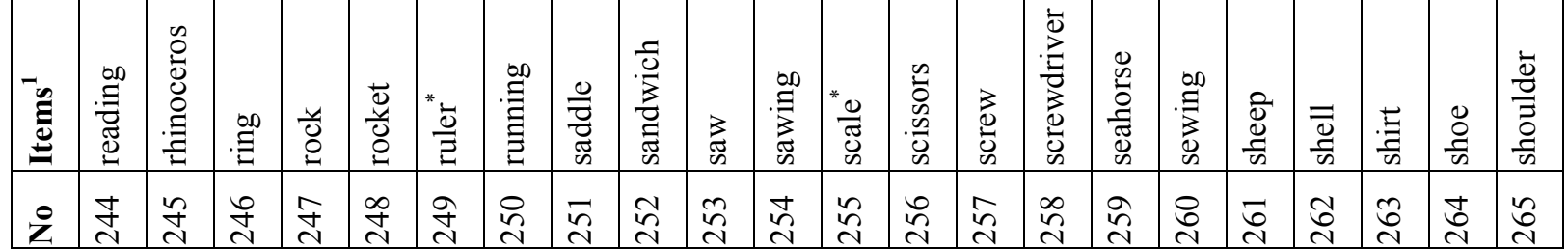




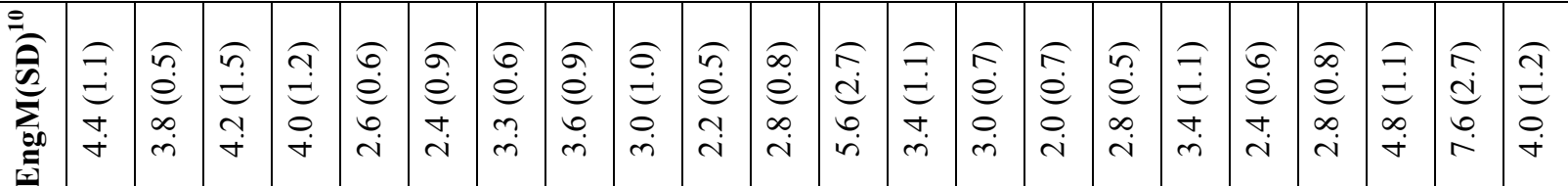

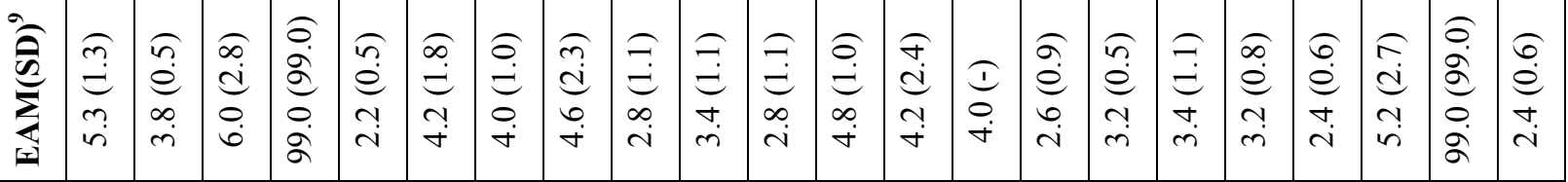

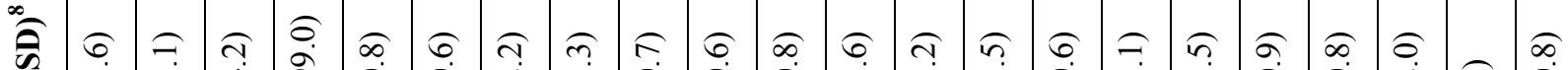

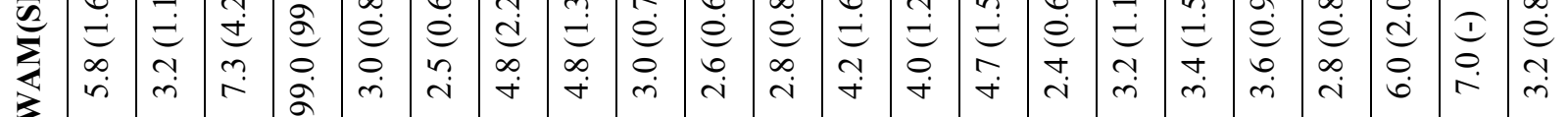

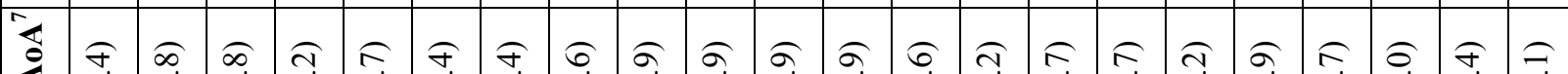

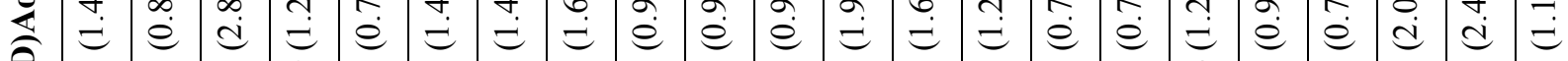

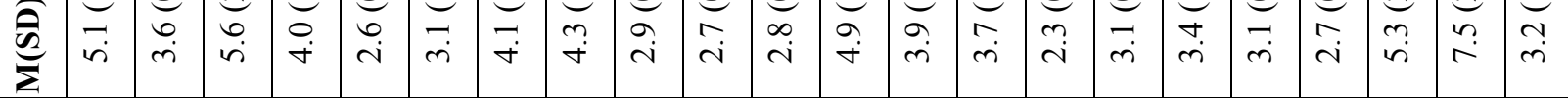

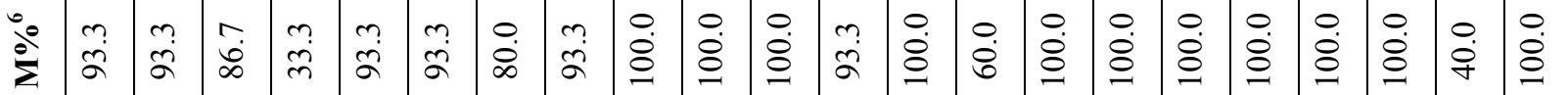

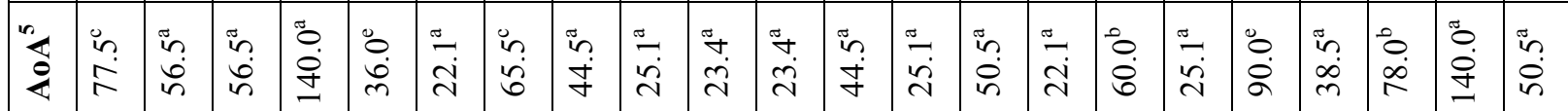

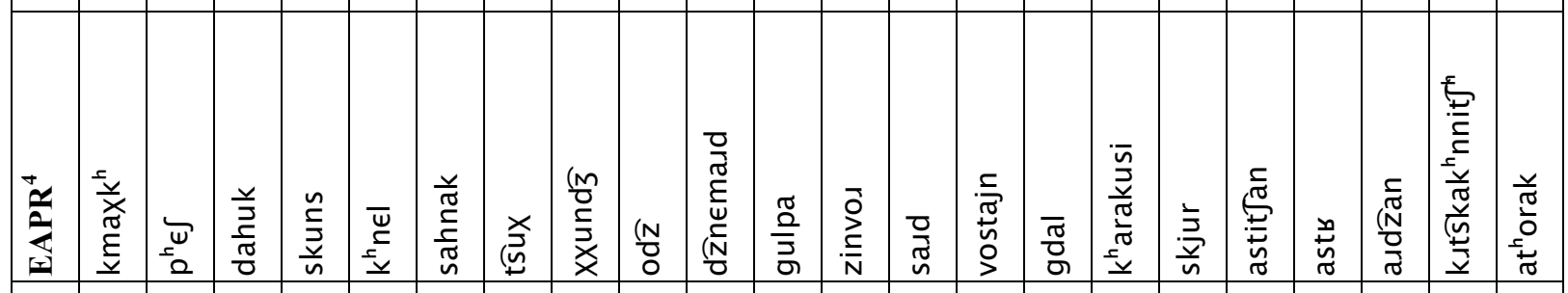

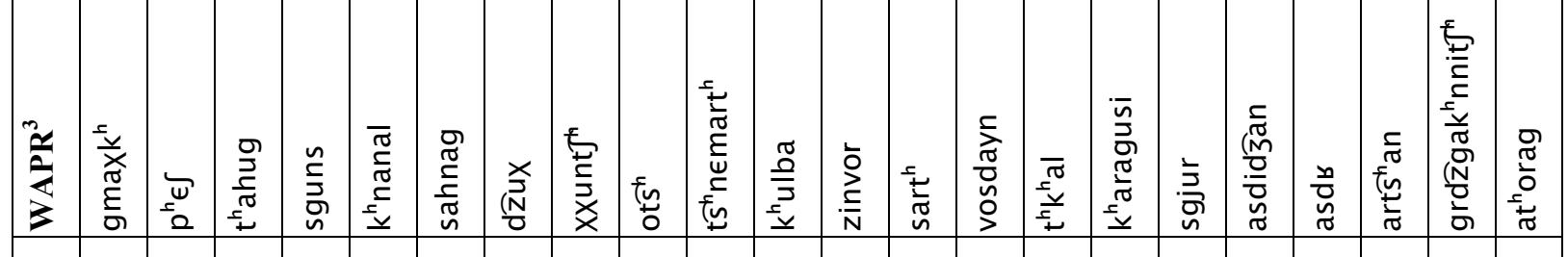

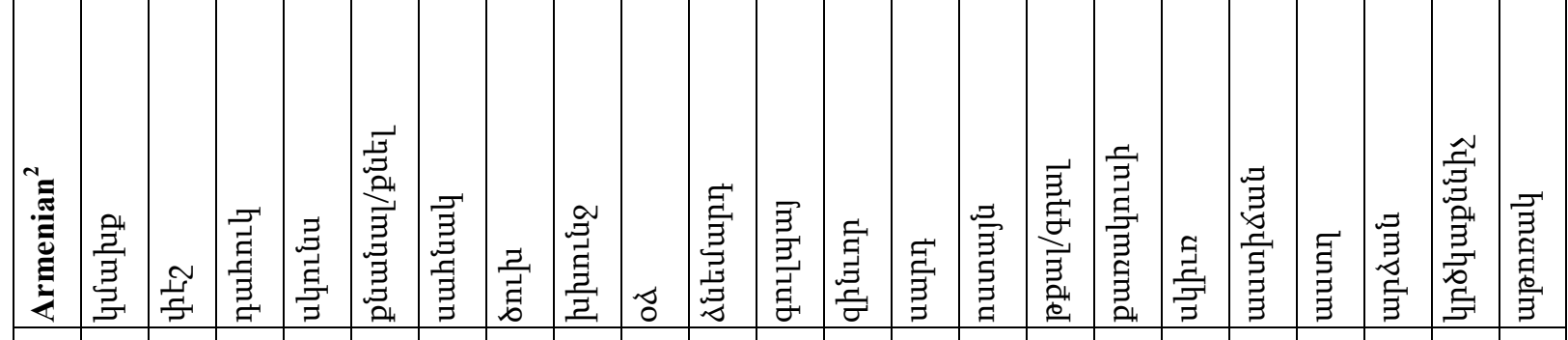

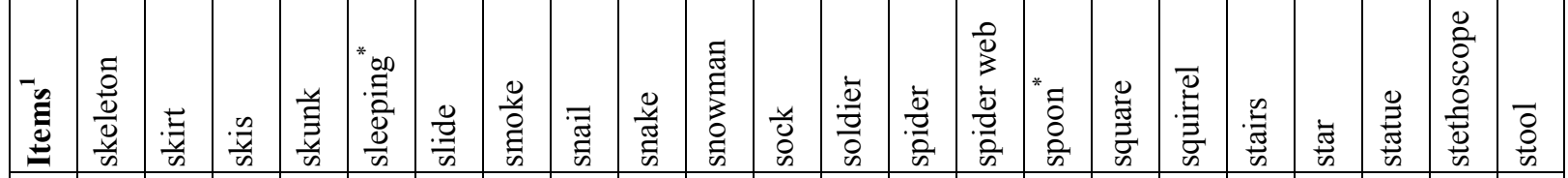

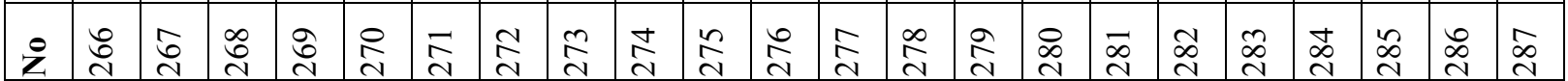




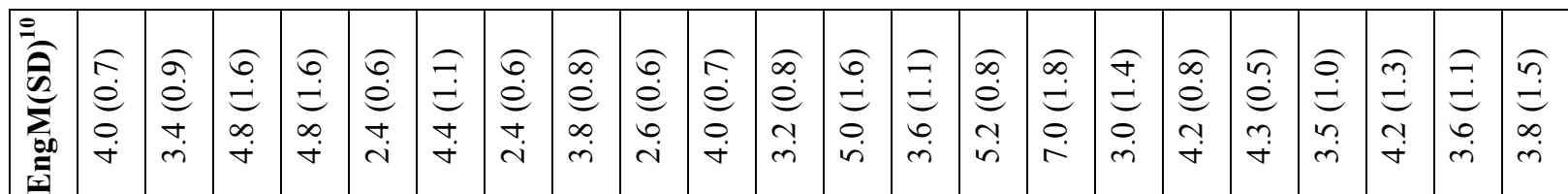

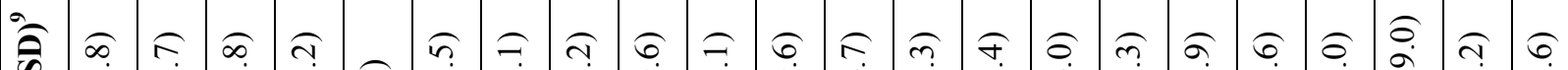

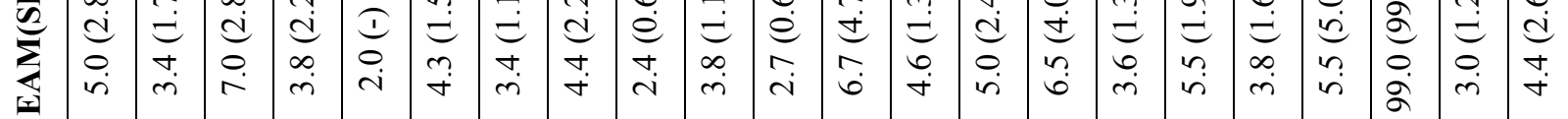

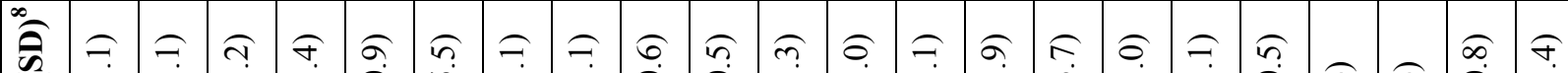

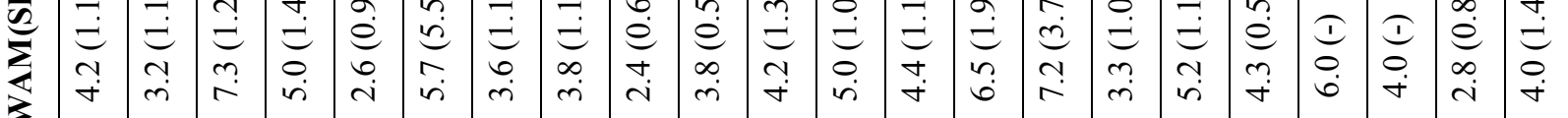

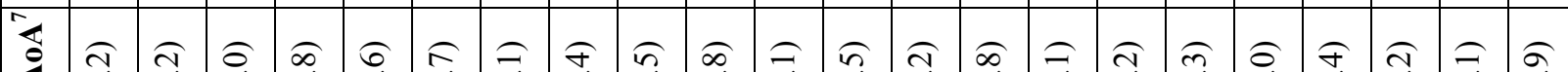

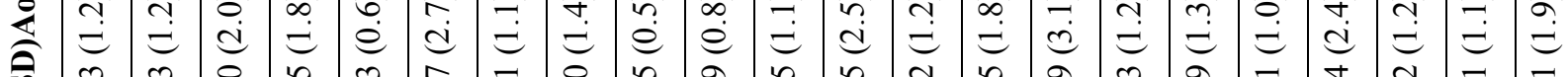

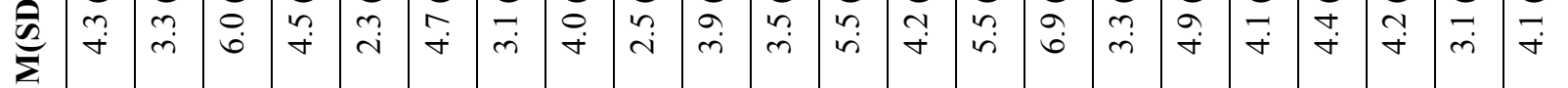

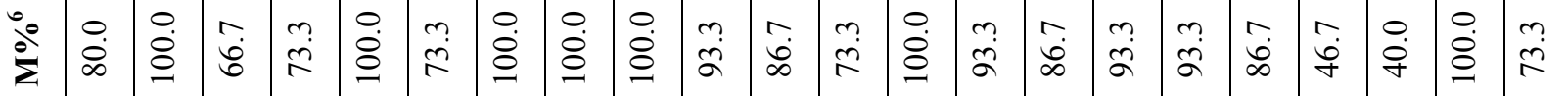

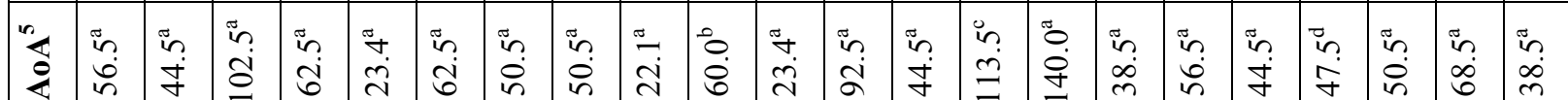

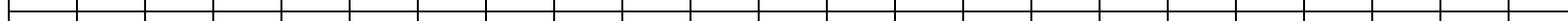

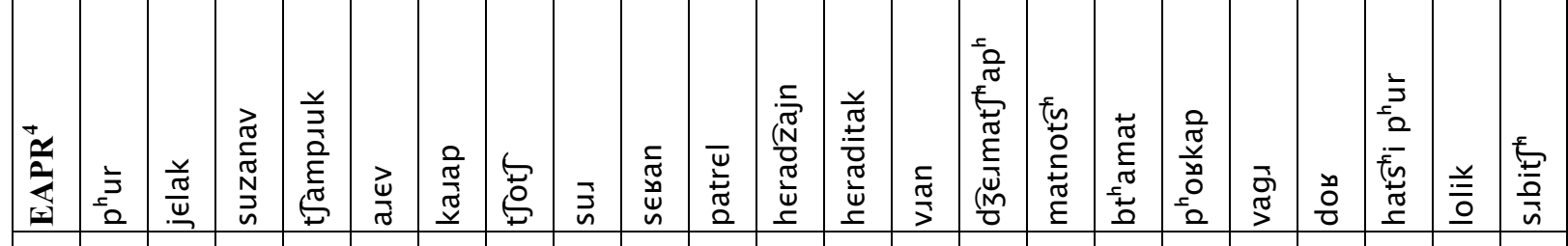

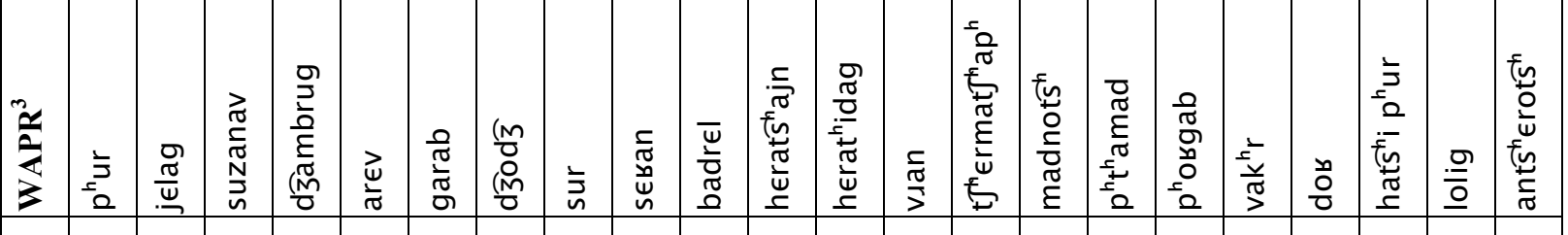

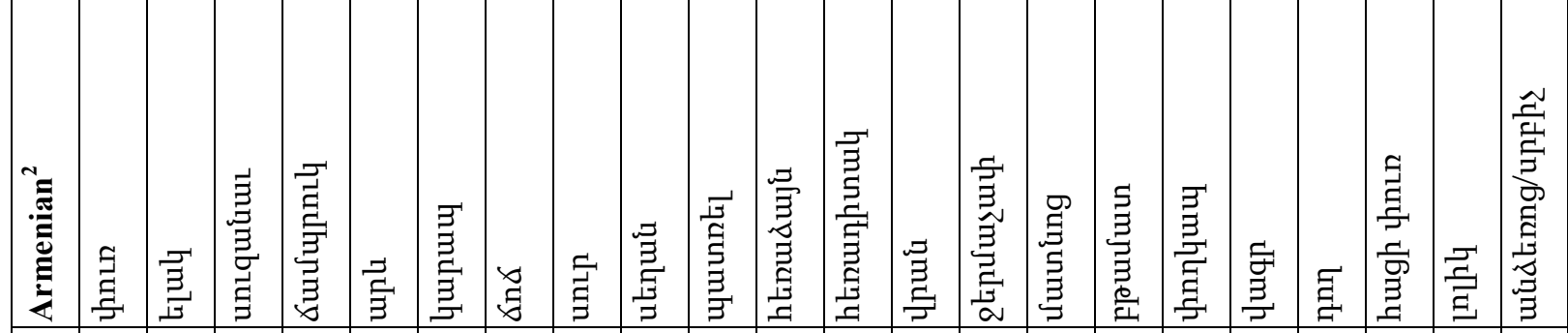

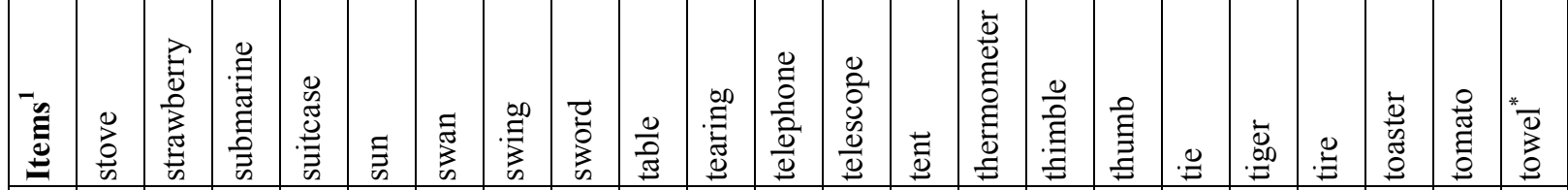

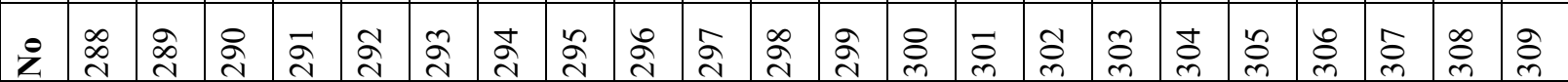




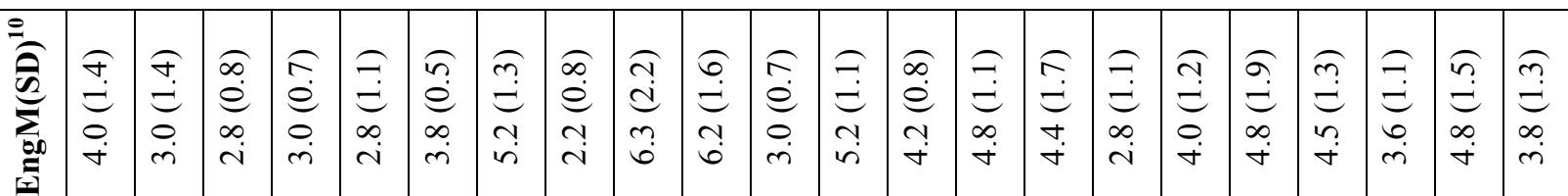

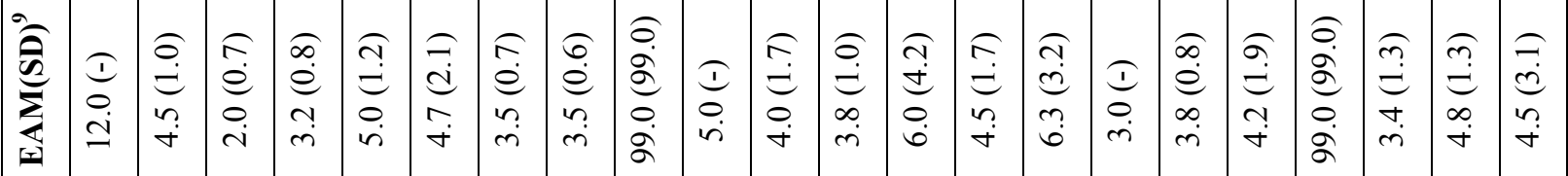

के

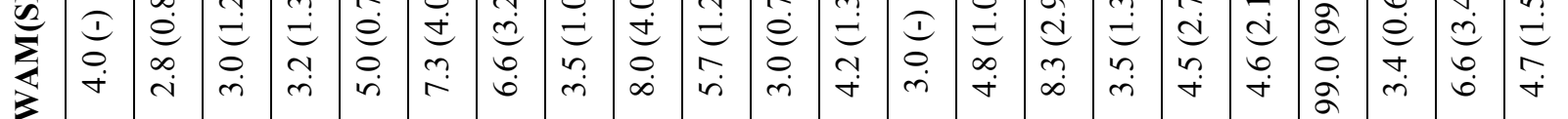

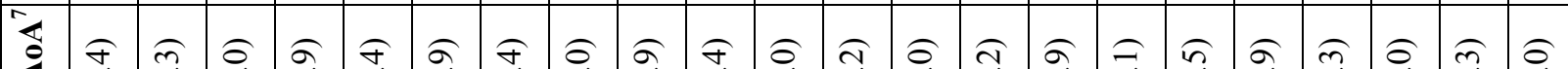

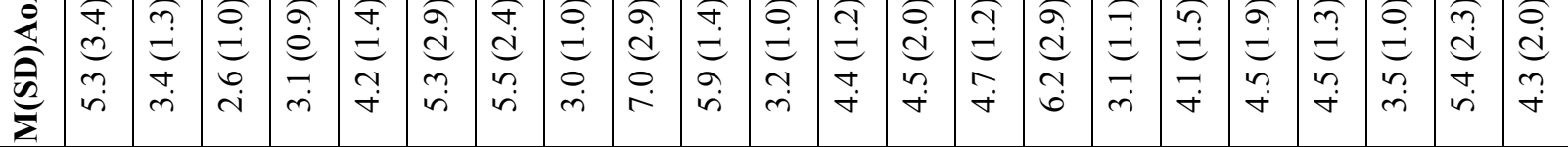

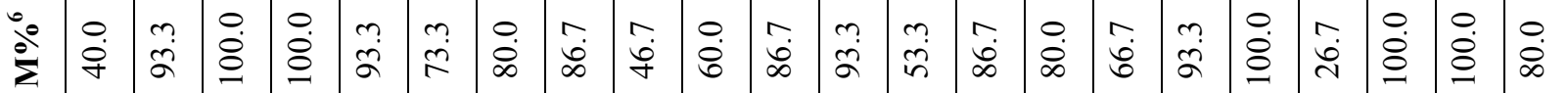

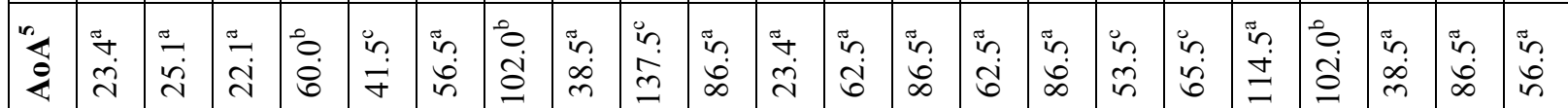

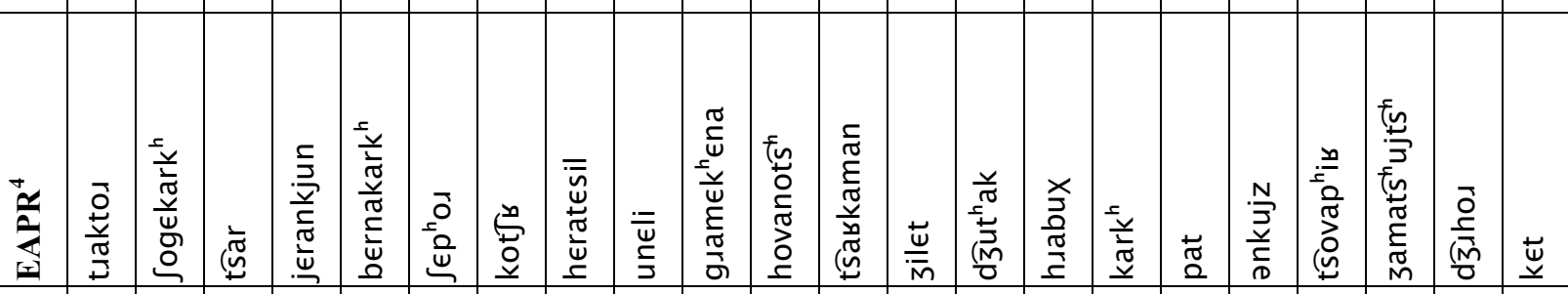

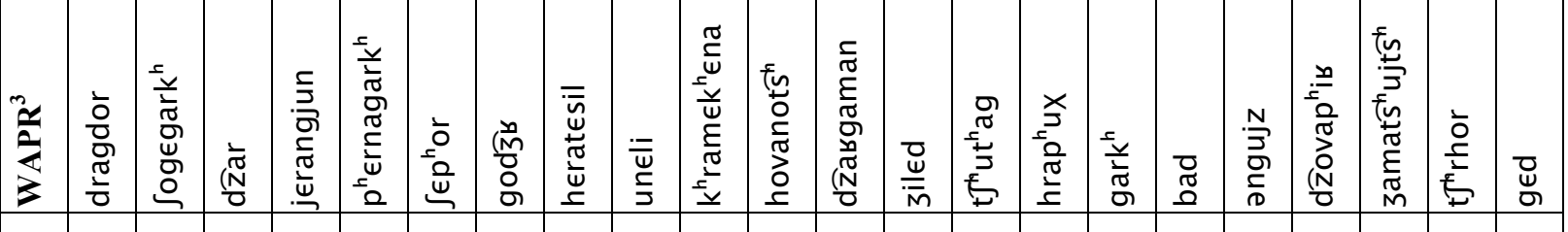

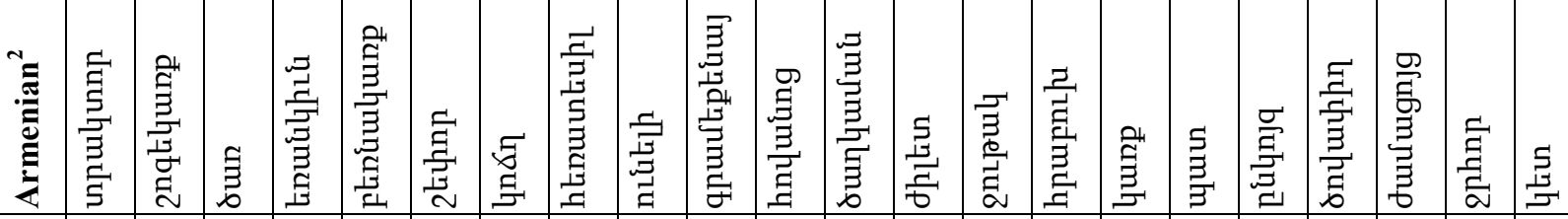

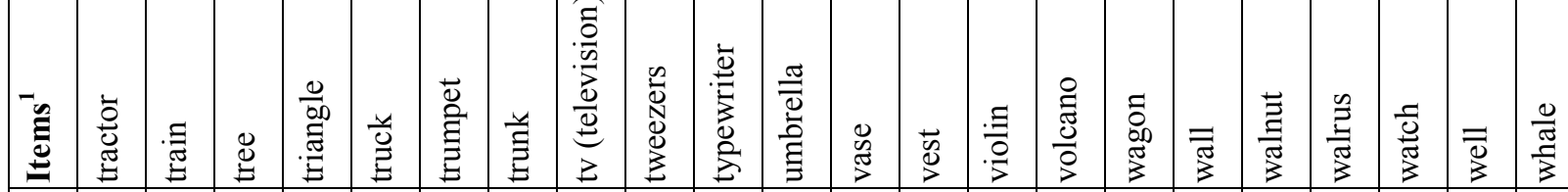

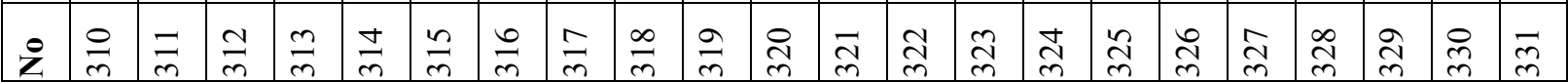


E

\begin{tabular}{|c|c|c|c|c|c|c|c|c|c|c|c|c|}
\hline$\sum_{\infty \infty \infty}^{\infty}$ & 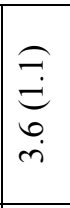 & 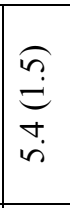 & 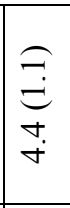 & 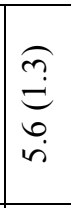 & $\begin{array}{l}\hat{\jmath} \\
\hat{e} \\
\dot{r} \\
\dot{n}\end{array}$ & $\mid \begin{array}{l}\hat{a} \\
\dot{e} \\
0 \\
\dot{n}\end{array}$ & 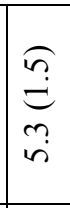 & $\begin{array}{l}\stackrel{\Im}{d} \\
\stackrel{d}{d} \\
\text { b. }\end{array}$ & 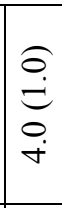 & 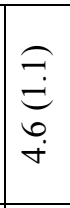 & $\mid \begin{array}{l}\stackrel{\sigma}{\dot{\Xi}} \\
\stackrel{0}{+} \\
\dot{+}\end{array}$ & $\mid \begin{array}{l}\hat{a} \\
\hat{e} \\
\dot{n} \\
\dot{n}\end{array}$ \\
\hline 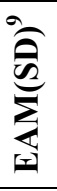 & 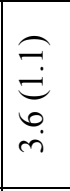 & $\begin{array}{l}I \\
\stackrel{1}{0} \\
\text { ind }\end{array}$ & $\begin{array}{l}\hat{\sigma} \\
\hat{e} \\
\underline{r} \\
\dot{r}\end{array}$ & $\begin{array}{l}\underset{d}{d} \\
\dot{d} \\
\stackrel{0}{i}\end{array}$ & $\begin{array}{l}\hat{\infty} \\
\stackrel{e}{e} \\
\infty \\
i\end{array}$ & $\begin{array}{l}\text { } \\
\stackrel{d}{d} \\
0 \\
\text { in }\end{array}$ & 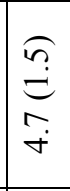 & $\begin{array}{l}\hat{\sigma} \\
\dot{a} \\
\hat{\sigma} \\
\dot{0} \\
\dot{\alpha}\end{array}$ & $\begin{array}{l}\tilde{n} \\
\hat{e} \\
\infty \\
i\end{array}$ & $\mid \begin{array}{l}\tilde{n} \\
e \\
\infty \\
\dot{n}\end{array}$ & $\begin{array}{l}\hat{\sigma} \\
\dot{a} \\
\hat{\sigma} \\
\circ \\
\dot{a} \\
\dot{a}\end{array}$ & $\begin{array}{l}1 \\
\\
0 \\
\infty\end{array}$ \\
\hline 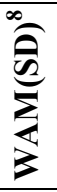 & $\begin{array}{l}\underset{6}{\infty} \\
\stackrel{\infty}{\infty} \\
\dot{m}\end{array}$ & $\begin{array}{l}\hat{\partial} \\
d \\
\dot{d} \\
\dot{i}\end{array}$ & 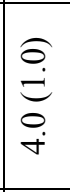 & 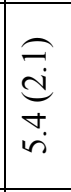 & 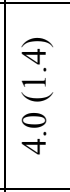 & 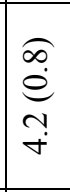 & 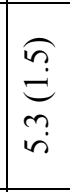 & $\begin{array}{l}\hat{\sigma} \\
\dot{a} \\
\hat{\sigma} \\
\dot{0} \\
\dot{\alpha}\end{array}$ & 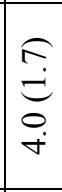 & 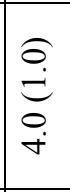 & 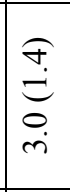 & \begin{tabular}{|l}
1 \\
1 \\
0 \\
0
\end{tabular} \\
\hline 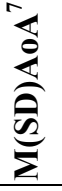 & 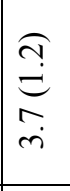 & 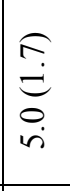 & 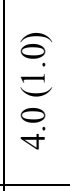 & 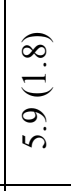 & 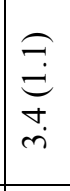 & 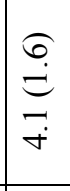 & 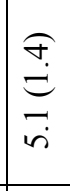 & 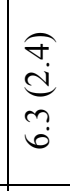 & 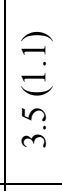 & $\begin{array}{l}\hat{\hat{g}} \\
\hat{e} \\
\stackrel{\gamma}{\sigma}\end{array}$ & 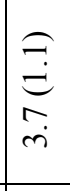 & $\begin{array}{l}\stackrel{\vec{d}}{d} \\
\vec{r}\end{array}$ \\
\hline$\stackrel{b}{i}^{\circ}$ & $\stackrel{\stackrel{\circ}{\dot{8}}}{ }$ & $\stackrel{\circ}{\dot{B}}$ & $\stackrel{\circ}{\dot{8}}$ & $\hat{\infty}$ & $\stackrel{m}{\sigma}$ & $\stackrel{\circ}{\stackrel{8}{9}}$ & $\stackrel{\circ}{0}$ & $\overrightarrow{\mathrm{d}}$ & $\stackrel{m}{n}$ & $\stackrel{\circ}{\dot{\infty}}$ & $\hat{\sigma}$ & $\hat{\grave{o}}$ \\
\hline$\overleftrightarrow{s}$ & 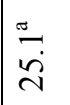 & \begin{tabular}{l} 
in \\
\hdashline \\
$f$
\end{tabular} & $\begin{array}{l}\text { in } \\
\text { in }\end{array}$ & $\begin{array}{l}\text { in } \\
\hat{n} \\
i n\end{array}$ & $\frac{\pi}{\ddot{n}}$ & $\begin{array}{l}\tilde{n} \\
i \\
i n \\
n\end{array}$ & $\begin{array}{l}\stackrel{0}{\circ} \\
\dot{\sigma}\end{array}$ & $\begin{array}{l}0 \\
\stackrel{0}{\mathrm{~d}} \\
\stackrel{0}{0}\end{array}$ & $\begin{array}{l}\therefore \\
\therefore \infty \\
\therefore\end{array}$ & $\stackrel{0}{\circ}$ & $\begin{array}{l}\text { in } \\
\stackrel{n}{i}\end{array}$ & in \\
\hline
\end{tabular}

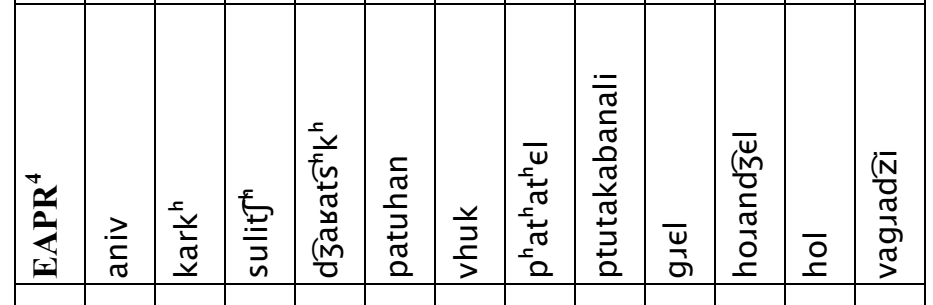

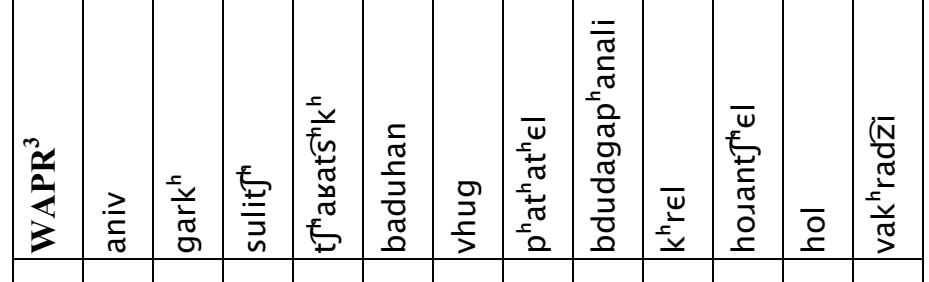
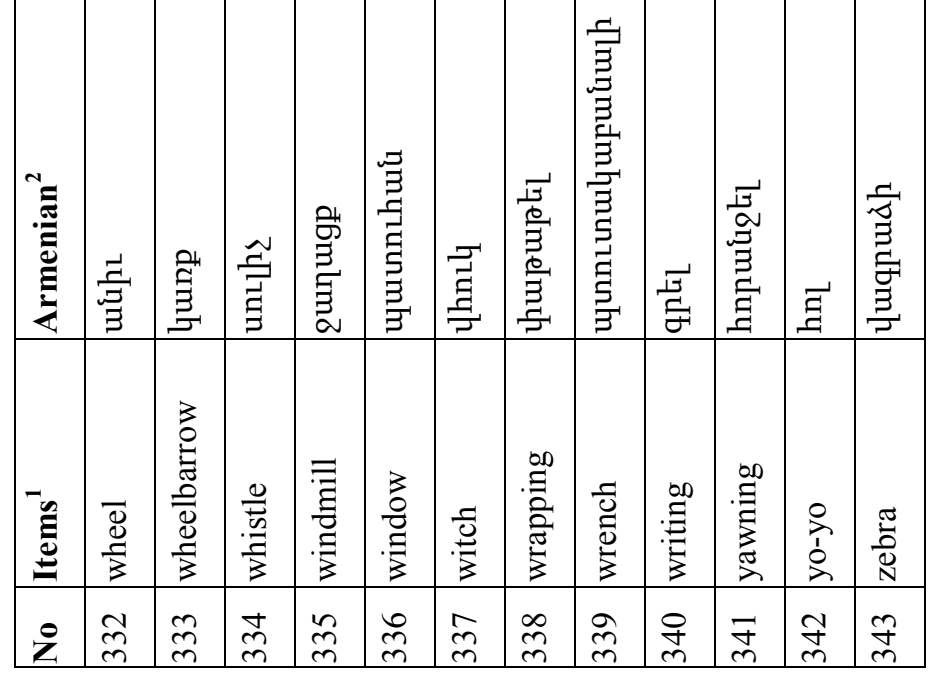


\section{Appendix C}

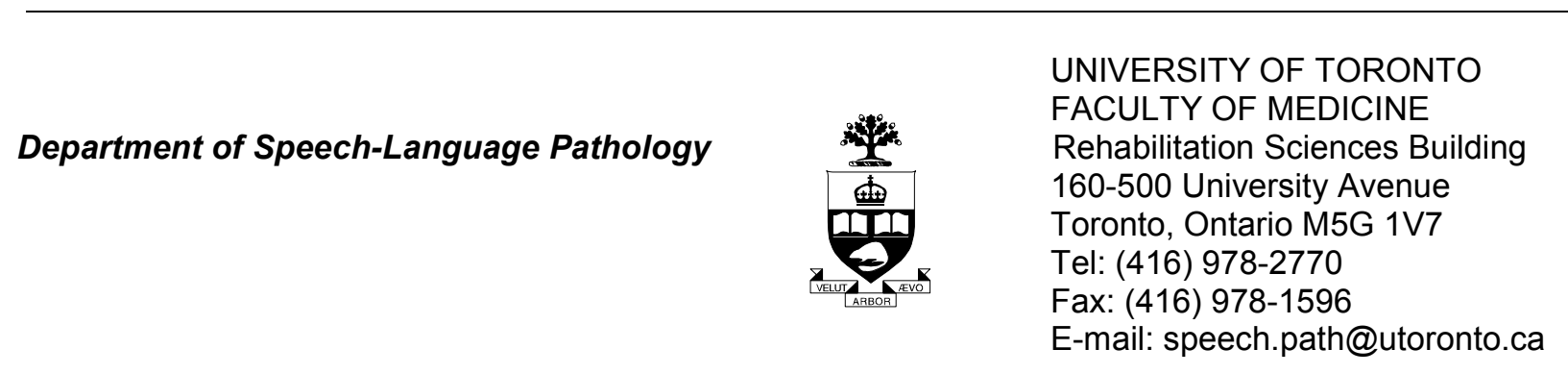

\section{Information Letter and Informed Consent Form for Teachers in Study 1}

Title of Research Project: Vocabulary Development in Armenian Children Attending Bilingual Preschools

Investigator: Alice Hovsepian, $\mathrm{PhD}$ Student

Department of Speech-Language Pathology, University of Toronto

The research project is designed as my $\mathrm{PhD}$ dissertation research under the supervision of Dr. Carla J. Johnson.

\section{Dear Teacher,}

This is a formal invitation to consider participating in this study. You are invited to participate in this study because you have the qualifications needed for this study. To participate in this study you must be a fluent speaker of either Western Armenian or Eastern Armenian, or a first language speaker of English and you must be familiar with young children.

The decision to participate in this study is totally voluntary. You can withdraw from the study at any time for any reason.

This study is the first part of the project. The purpose of this study is to develop a vocabulary test for preschool Armenian children. By developing this test we will be able to study the development of English and Armenian vocabulary in Armenian children. Overall about 15 teachers and parents are required to take part in this study. Five of them will be fluent speakers of Western Armenian, five will be fluent speakers of Eastern Armenian, and another five will be first-language speakers of English.

Upon giving your consent to participate in this study, we will arrange a 1-hour meeting in the school or another acceptable location and at a time convenient for you. The study will involve two tasks. In the first task you will be shown simple line-drawing pictures one at a time on a laptop computer and will be asked to name them. You will speak your responses into the microphone attached to the laptop, and your responses will be recorded. In the second task, after naming each picture you will be asked to rate each name for the age you think children learn the name. For this part, you will choose one of the ages from the options provided on the laptop screen. 
Participation in this study provides no direct benefits and presents no foreseeable risks. The only inconvenience that you might face is feeling tired. If so, you can choose to have a short break and continue with the task after the break.

All your personal information is confidential. I will use a code for identifying you in my database and there will be no mention of your name anywhere. All of your voice and text responses will be saved on the lab computer on a space protected by password. The information recorded on paper will be kept in a locked file in my supervisor's lab. No other person except me, my supervisor and possibly a research assistant will have access to this information. All of this information will be destroyed completely after five years following publication of the study. The paper data will be shredded and the computer data will be completely deleted.

The results obtained from all participants in this study will be reported as group findings in future publications with no mention of names or any other personal information. A summary of the results of the project will be prepared and sent to you.

If you have any further questions you can contact me, Alice Hovsepian, by phone at 416-9468638 or by e-mail at alice.hovsepian@utoronto.ca. For information regarding your rights as a participant you can contact the University of Toronto Ethics Review Office by phone at 416-9463273 or by e-mail at ethics.review@utoronto.ca.

If you decide to participate, please print your name, sign and date the last line of one of the two copies of this form, and return it to me in the envelope provided with the form. Please keep the second copy of the form for your own reference.

\section{Investigator's Statement}

I have fully explained this study to the teacher. I have discussed the activities and have answered all the questions that the teacher asked.

Signature of the Investigator Date:

\section{Participant's Consent}

I have read the information provided in this Informed Consent Form. All my questions have been answered satisfactorily. I agree to prticipate in this study.

Name:

Your Signature

Date: 
Department of Speech-Language Pathology

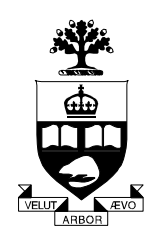

UNIVERSITY OF TORONTO

FACULTY OF MEDICINE

Rehabilitation Sciences Building

160-500 University Avenue

Toronto, Ontario M5G 1V7

Tel: (416) 978-2770

Fax: (416) 978-1596

E-mail: speech.path@utoronto.ca

\section{Information Letter and Informed Consent Form for Parents in Study 1}

Title of Research Project: Vocabulary Development in Armenian Children Attending Bilingual Preschools

Investigator: Alice Hovsepian, PhD Student

Department of Speech-Language Pathology, University of Toronto

The research project is designed as my $\mathrm{PhD}$ dissertation research under the supervision of $\mathrm{Dr}$. Carla J. Johnson.

\section{Dear Parent,}

This is a formal invitation to consider participating in this study. You are invited to participate in this study because you have the qualifications needed for this study. To participate in this study you must be a fluent speaker of either Western Armenian or Eastern Armenian, or a first language speaker of English and you must be familiar with young children.

The decision to participate in this study is totally voluntary. You can withdraw from the study at any time for any reason.

This study is the first part of the project. The purpose of this study is to develop a vocabulary test for preschool Armenian children. By developing this test we will be able to study the development of the English and Armenian vocabulary in Armenian children. Overall about 15 parents and teachers are required to participate in this study. Five of them will be fluent speakers of Western Armenian, five will be fluent speakers of Eastern Armenian, and another five will be first language speakers of English.

Upon giving your consent to participate in this study, we will arrange a 1-hour meeting in the school or another acceptable location and at a time convenient for you. The study will involve two tasks. In the first task you will be shown simple line-drawing pictures one at a time on a laptop computer and will be asked to name them. You will speak your responses into the microphone attached to the laptop, and your responses will be recorded. In the second task, after naming each picture you will be asked to rate each name for the age you think children learn the name. For this part, you will choose one of the ages from the options provided on the laptop screen. 
Participation in this study provides no direct benefits and presents no foreseeable risks. The only inconvenience that you might face is feeling tired. If so, you can choose to have a short break and continue with the task after the break.

All your personal information is confidential. I will use a code for identifying you in my database and there will be no mention of your name anywhere. All of your voice and text responses will be saved on the lab computer on a space protected by password. The information recorded on paper will be kept in a locked file in my supervisor's lab. No other person except me, my supervisor and possibly a research assistant will have access to this information. All of this information will be destroyed completely after five years following publication of the study. The paper data will be shredded and the computer data will be completely deleted.

The results obtained from all participants in this study will be reported as group findings in future publications with no mention of the names or any other personal information. A summary of the results of the project will be prepared and sent to you.

If you have any further questions you can contact me, Alice Hovsepian, by phone at 416-9468638 or by e-mail at alice.hovsepian@utoronto.ca. For information regarding your rights as a participant you can contact the University of Toronto Ethics Review Office by phone at 416-9463273 or by e-mail at ethics.review@utoronto.ca.

If you decide to participate; please print your name, provide your phone number, sign and date the last line of one of the two copies of this form, put it in the envelope provided with the form, seal the envelope, and give the envelope to your child to return it to the teacher who will pass it to me unopened. Please keep the second copy of the form for your own reference.

\section{Investigator's Statement}

I have fully explained this study to the parent. I have discussed the activities and have answered all the questions that the parent asked.

Signature of the Investigator

Date:

\section{Participant's Consent}

I have read the information provided in this Informed Consent Form. All my questions have been answered satisfactorily. I agree to prticipate in this study.

Name:

Your Phone Number:

Your Signature

Date: 


\section{Appendix D}

Table 1

\section{Four Lists of Selected Vocabulary Items}

All Target Items in the Four Lists (A, B, C, and D) in the Order in Which They Appeared on the Tasks

\begin{tabular}{|c|c|c|c|c|}
\hline No & List A & List B & List C & List D \\
\hline 1 & cat & apple & $\operatorname{dog}$ & ball \\
\hline 2 & nose & shoe & sun & door \\
\hline 3 & table & book & cheese & chair \\
\hline 4 & ear & rabbit & fish & horse \\
\hline 5 & cow & balloon & duck & drinking \\
\hline 6 & girl & flower & pencil & tree \\
\hline 7 & eye & star & fork & bird \\
\hline 8 & pig & bed & hat & snowman \\
\hline 9 & sock & heart & butterfly & leaf \\
\hline 10 & monkey & airplane & elephant & moon \\
\hline 11 & bell & button & snake & bear \\
\hline 12 & pants & candle & drum & square \\
\hline 13 & stairs & swing & tomato & box \\
\hline 14 & boat & grapes & flag & triangle \\
\hline 15 & glasses & stool & bicycle & scissors \\
\hline 16 & pear & church & key & knife \\
\hline 17 & orange & strawberry & lion & bottle \\
\hline 18 & squirrel & comb & donkey & ring \\
\hline 19 & watch & pumpkin & belt & dress \\
\hline 20 & fridge & clown & lemon & broom \\
\hline 21 & king & nail (finger) & basket & wheel \\
\hline 22 & spider & hammer & crown & giraffe \\
\hline 23 & bow (ribbon) & goat & canoe & needle \\
\hline 24 & paintbrush & sword & guitar & mountain \\
\hline
\end{tabular}




\begin{tabular}{|l|l|l|l|l|}
\hline No & List A & List B & List C & List D \\
\hline 25 & whistle & wall & witch & camel \\
\hline 26 & coat & potato & owl & tiger \\
\hline 27 & pepper & mushroom & smoke & lamp \\
\hline 28 & necklace & couch & queen & nest \\
\hline 29 & yawning & tent & chain & dentist \\
\hline 30 & truck & whale & stove & peeling \\
\hline 31 & fence & arrow & snail & drawer \\
\hline 32 & deer & rock & gorilla & shoulder \\
\hline 33 & vase & branch & onion & kettle \\
\hline 34 & sewing & suitcase & bridge & walnut \\
\hline 35 & saw & digging & iron & envelope \\
\hline 36 & swan & violin & rocket & kangaroo \\
\hline 37 & pan & antlers & pineapple & paw \\
\hline 38 & porcupine & desk & castle & leopard \\
\hline 39 & soldier & frame & lettuce & tie \\
\hline 40 & rhinoceros & skeleton & brain & wrapping \\
\hline 41 & faucet & merry-go-round & trumpet & pitcher \\
\hline 42 & palm tree & nurse & statue & lobster \\
\hline 43 & acorn & cage & well & ambulance \\
\hline 44 & ostrich & thermometer & telescope & crab \\
\hline 45 & trunk & globe & medal & skis \\
\hline 46 & peacock & sucrophone & calculator & sawing \\
\hline 47 & eagle & solcanor & windmill & cactus \\
\hline 48 & diamond & bow & flute \\
\hline 49 & ashtray & cigarette & fountain \\
\hline 50 & thimble & pillar & hoof \\
\hline 51 & diving & blender & rectangle \\
\hline 52 & oval & terrified & tornado \\
\hline 53 & pot & flask & anvil \\
\hline 54 & allipers & accordion & tape \\
\hline 55 & tambourine \\
\hline
\end{tabular}


Table 2

List A Target Items (in Bold) and Their Foils with Their Positions in the Four Corners of each Picture Identification Trial Page

\begin{tabular}{|c|c|c|c|c|}
\hline No & Corner 1 & Corner 2 & Corner 3 & Corner 4 \\
\hline 1 & bread & cat & hand & spoon \\
\hline 2 & glass & hair & nose & house \\
\hline 3 & banana & finger & table & sleeping \\
\hline 4 & mitten & ear & boot & car \\
\hline 5 & boy & cow & eating & foot \\
\hline 6 & frog & cake & girl & mouse \\
\hline 7 & chicken & television & eye & glove \\
\hline 8 & slide & carrot & doctor & pig \\
\hline 9 & wagon & sock & panda & plate \\
\hline 10 & bus & monkey & running & reading \\
\hline 11 & bowl & cup & cloud & bell \\
\hline 12 & pants & umbrella & panda & sandwich \\
\hline 13 & stairs & leg & thumb & lips \\
\hline 14 & painting & boat & train & kite \\
\hline 15 & shell & bee & window & glasses \\
\hline 16 & pear & caterpillar & barn & peanut \\
\hline 17 & writing & orange & coin & garbage can \\
\hline 18 & clock & brush & squirrel & telephone \\
\hline 19 & needle (syringe) & bee & jacket & watch \\
\hline 20 & fridge & skirt & sheep & clapping \\
\hline 21 & spider web & lamb & shirt & king \\
\hline 22 & spider & corn & bat & fox \\
\hline 23 & bow (ribbon) & yo-yo & jumping & cherry \\
\hline 24 & penguin & paintbrush & ant & bathtub \\
\hline 25 & pen & whistle & ladder & shirt \\
\hline 26 & purse & coat & dragon & gun \\
\hline 27 & fly & pepper & camera & closet \\
\hline 28 & tearing & necklace & piano & peach \\
\hline
\end{tabular}




\begin{tabular}{|c|c|c|c|c|}
\hline No & Corner 1 & Corner 2 & Corner 3 & Corner 4 \\
\hline 29 & pulling & helicopter & feather & yawning \\
\hline 30 & motorcycle & cowboy & truck & ladybug \\
\hline 31 & fence & net & fly & nail \\
\hline 32 & skunk & towel & octopus & deer \\
\hline 33 & zebra & ruler & light bulb & vase \\
\hline 34 & sewing & octopus & plug & toaster \\
\hline 35 & fire hydrant & saw & arm & seahorse \\
\hline 36 & raccoon & mermaid & swan & tire \\
\hline 37 & pan & walrus & armadillo & vest \\
\hline 38 & porcupine & hook & astronaut & beetle \\
\hline 39 & parachute & screwdriver & fire hydrant & soldier \\
\hline 40 & wheelbarrow & pyramid & rhinoceros & biscuit \\
\hline 41 & celery & drilling & faucet & screw \\
\hline 42 & saddle & palm tree & tractor & pelican \\
\hline 43 & climbing & axe & binoculars & acorn \\
\hline 44 & claw & lock & ostrich & scale \\
\hline 45 & typewriter & barrel & tractor & trunk \\
\hline 46 & chisel & peacock & flamingo & hyena \\
\hline 47 & harp & eagle & pliers & wrench \\
\hline 48 & fern & pipe & diamond & cymbals \\
\hline 49 & ashtray & propeller & funnel & tweezers \\
\hline 50 & microscope & thimble & bug & stethoscope \\
\hline 51 & diving & fingerprint & banjo & igloo \\
\hline 52 & skull & saxophone & cap & oval \\
\hline 53 & pot & hourglass & squash & floating \\
\hline 54 & tuning fork & tape recorder & skateboard & callipers \\
\hline 55 & battery & sorting & accordion & towing \\
\hline
\end{tabular}


Table 3

List B Target Items (in Bold) and Their Foils with Their Positions in the Four Corners of each

Picture Identification Trial Page

\begin{tabular}{|c|c|c|c|c|}
\hline No & Corner 1 & Corner 2 & Corner 3 & Corner 4 \\
\hline 1 & apple & car & sleeping & glass \\
\hline 2 & bread & mitten & shoe & finger \\
\hline 3 & boot & book & hair & hand \\
\hline 4 & banana & rabbit & house & spoon \\
\hline 5 & balloon & doctor & television & cake \\
\hline 6 & carrot & glove & flower & eating \\
\hline 7 & mouse & boy & star & slide \\
\hline 8 & frog & chicken & bed & foot \\
\hline 9 & bus & cup & umbrella & heart \\
\hline 10 & cloud & sandwich & wagon & airplane \\
\hline 11 & panda & plate & button & running \\
\hline 12 & panda & reading & bowl & candle \\
\hline 13 & painting & bee & peanut & swing \\
\hline 14 & barn & thumb & shell & grapes \\
\hline 15 & lips & caterpillar & stool & kite \\
\hline 16 & church & leg & window & train \\
\hline 17 & telephone & skirt & jacket & strawberry \\
\hline 18 & needle (syringe) & garbage can & sheep & comb \\
\hline 19 & brush & coin & pumpkin & clapping \\
\hline 20 & bee & writing & clown & clock \\
\hline 21 & ant & cherry & bat & nail (finger) \\
\hline 22 & penguin & hammer & spider web & yo-yo \\
\hline 23 & bathtub & goat & corn & lamb \\
\hline 24 & shirt & jumping & fox & sword \\
\hline 25 & purse & closet & tearing & wall \\
\hline 26 & potato & piano & pen & camera \\
\hline 27 & peach & shirt & dragon & mushroom \\
\hline 28 & couch & fly & gun & ladder \\
\hline
\end{tabular}




\begin{tabular}{|c|c|c|c|c|}
\hline No & Corner 1 & Corner 2 & Corner 3 & Corner 4 \\
\hline 29 & fly & cowboy & skunk & tent \\
\hline 30 & whale & octopus & net & helicopter \\
\hline 31 & towel & pulling & ladybug & arrow \\
\hline 32 & feather & rock & nail & motorcycle \\
\hline 33 & branch & raccoon & fire hydrant & toaster \\
\hline 34 & mermaid & arm & suitcase & zebra \\
\hline 35 & ruler & tire & plug & digging \\
\hline 36 & violin & light bulb & octopus & seahorse \\
\hline 37 & hook & screwdriver & antlers & pyramid \\
\hline 38 & armadillo & desk & biscuit & parachute \\
\hline 39 & wheelbarrow & beetle & frame & vest \\
\hline 40 & skeleton & walrus & fire hydrant & astronaut \\
\hline 41 & binoculars & merry-go-round & pelican & lock \\
\hline 42 & axe & nurse & drilling & scale \\
\hline 43 & celery & cage & saddle & claw \\
\hline 44 & tractor & thermometer & climbing & screw \\
\hline 45 & fern & globe & chisel & pliers \\
\hline 46 & microphone & typewriter & cymbals & wrench \\
\hline 47 & pipe & anchor & tractor & flamingo \\
\hline 48 & submarine & harp & barrel & hyena \\
\hline 49 & tweezers & volcano & funnel & propeller \\
\hline 50 & cannon & microscope & bug & stethoscope \\
\hline 51 & fingerprint & igloo & dripping & banjo \\
\hline 52 & cap & skull & saxophone & scorpion \\
\hline 53 & sled & hourglass & floating & squash \\
\hline 54 & skateboard & tuning fork & tape recorder & knight \\
\hline 55 & sorting & battery & compass & towing \\
\hline
\end{tabular}


Table 4

List C Target Items (in Bold) and Their Foils in with Their Positions in the Four Corners of each

Picture Identification Trial Page

\begin{tabular}{|c|c|c|c|c|}
\hline No & Corner 1 & Corner 2 & Corner 3 & Corner 4 \\
\hline 1 & mitten & $\operatorname{dog}$ & sleeping & hair \\
\hline 2 & banana & sun & car & bread \\
\hline 3 & boot & spoon & cheese & glass \\
\hline 4 & fish & house & hand & finger \\
\hline 5 & duck & television & carrot & mouse \\
\hline 6 & pencil & chicken & eating & doctor \\
\hline 7 & cake & fork & slide & foot \\
\hline 8 & frog & glove & hat & boy \\
\hline 9 & running & cup & sandwich & butterfly \\
\hline 10 & bowl & umbrella & wagon & elephant \\
\hline 11 & snake & panda & window & bus \\
\hline 12 & drum & plate & cloud & reading \\
\hline 13 & kite & tomato & barn & bee \\
\hline 14 & peanut & panda & flag & thumb \\
\hline 15 & painting & bicycle & caterpillar & leg \\
\hline 16 & shell & lips & key & train \\
\hline 17 & jacket & lion & sheep & brush \\
\hline 18 & skirt & bee & donkey & garbage can \\
\hline 19 & clapping & belt & writing & telephone \\
\hline 20 & coin & needle (syringe) & lemon & clock \\
\hline 21 & penguin & bathtub & basket & corn \\
\hline 22 & ant & jumping & crown & spider web \\
\hline 23 & canoe & cherry & shirt & bat \\
\hline 24 & fox & yo-yo & lamb & guitar \\
\hline 25 & dragon & closet & piano & witch \\
\hline 26 & tearing & fly & owl & pen \\
\hline 27 & smoke & ladder & peach & purse \\
\hline 28 & camera & gun & queen & shirt \\
\hline
\end{tabular}




\begin{tabular}{|c|c|c|c|c|}
\hline No & Corner 1 & Corner 2 & Corner 3 & Corner 4 \\
\hline 29 & octopus & fly & ladybug & chain \\
\hline 30 & nail & skunk & helicopter & stove \\
\hline 31 & cowboy & feather & snail & towel \\
\hline 32 & gorilla & motorcycle & pulling & net \\
\hline 33 & fire hydrant & plug & onion & mermaid \\
\hline 34 & zebra & seahorse & bridge & raccoon \\
\hline 35 & iron & toaster & tire & light bulb \\
\hline 36 & arm & rocket & octopus & ruler \\
\hline 37 & biscuit & beetle & pineapple & screwdriver \\
\hline 38 & armadillo & pyramid & castle & fire hydrant \\
\hline 39 & wheelbarrow & walrus & hook & lettuce \\
\hline 40 & astronaut & vest & parachute & brain \\
\hline 41 & saddle & scale & binoculars & trumpet \\
\hline 42 & drilling & statue & lock & climbing \\
\hline 43 & well & claw & pelican & screw \\
\hline 44 & tractor & celery & axe & telescope \\
\hline 45 & medal & cymbals & pliers & flamingo \\
\hline 46 & fern & calculator & typewriter & harp \\
\hline 47 & barrel & pipe & windmill & chisel \\
\hline 48 & wrench & hyena & bow & tractor \\
\hline 49 & propeller & cigarette & tweezers & funnel \\
\hline 50 & bug & microscope & pillar & stethoscope \\
\hline 51 & fingerprint & banjo & blender & igloo \\
\hline 52 & cap & skull & terrified & saxophone \\
\hline 53 & flask & floating & squash & hourglass \\
\hline 54 & island & tape recorder & tuning fork & skateboard \\
\hline 55 & towing & battery & nut & sorting \\
\hline
\end{tabular}


Table 5

List D Target Items (in Bold) and Their Foils with Their Positions in the Four Corners of each

Picture Identification Trial Page

\begin{tabular}{|c|c|c|c|c|}
\hline No & Corner 1 & Corner 2 & Corner 3 & Corner 4 \\
\hline 1 & ball & house & finger & mitten \\
\hline 2 & hand & sleeping & door & car \\
\hline 3 & banana & chair & bread & hair \\
\hline 4 & glass & spoon & horse & boot \\
\hline 5 & frog & glove & carrot & drinking \\
\hline 6 & doctor & tree & boy & television \\
\hline 7 & bird & cake & mouse & eating \\
\hline 8 & chicken & foot & slide & snowman \\
\hline 9 & sandwich & leaf & cloud & reading \\
\hline 10 & umbrella & moon & cup & plate \\
\hline 11 & bear & bowl & running & wagon \\
\hline 12 & square & lips & panda & bus \\
\hline 13 & shell & barn & train & box \\
\hline 14 & peanut & triangle & bee & panda \\
\hline 15 & thumb & kite & scissors & window \\
\hline 16 & leg & caterpillar & painting & knife \\
\hline 17 & clock & bottle & needle (syringe) & sheep \\
\hline 18 & ring & jacket & coin & skirt \\
\hline 19 & brush & garbage can & dress & bee \\
\hline 20 & telephone & broom & clapping & writing \\
\hline 21 & wheel & penguin & fox & уо-уо \\
\hline 22 & ant & lamb & cherry & giraffe \\
\hline 23 & spider web & needle & corn & jumping \\
\hline 24 & mountain & bat & bathtub & shirt \\
\hline 25 & camel & camera & piano & gun \\
\hline 26 & shirt & tiger & closet & tearing \\
\hline 27 & dragon & lamp & fly & pen \\
\hline
\end{tabular}




\begin{tabular}{|l|l|l|l|l|}
\hline No & Corner 1 & Corner 2 & Corner 3 & Corner 4 \\
\hline 28 & peach & ladder & nest & purse \\
\hline 29 & motorcycle & octopus & dentist & net \\
\hline 30 & skunk & peeling & pulling & fly \\
\hline 31 & ladybug & nail & helicopter & drawer \\
\hline 32 & feather & towel & cowboy & shoulder \\
\hline 33 & arm & mermaid & octopus & kettle \\
\hline 34 & ruler & fire hydrant & walnut & raccoon \\
\hline 35 & plug & zebra & envelope & seahorse \\
\hline 36 & kangaroo & light bulb & toaster & tire \\
\hline 37 & paw & astronaut & parachute & biscuit \\
\hline 38 & vest & screwdriver & leopard & pyramid \\
\hline 39 & armadillo & tie & beetle & fire hydrant \\
\hline 40 & hook & walrus & wheelbarrow & wrapping \\
\hline 41 & pitcher & scale & tractor & axe \\
\hline 42 & binoculars & celery & lock & lobster \\
\hline 43 & saddle & climbing & ambulance & drilling \\
\hline 44 & crab & screw & pelican & claw \\
\hline 45 & cymbals & wrench & skis & hyena \\
\hline 46 & sawing & tractor & pliers & fern \\
\hline 47 & flamingo & cactus & harp & typewriter \\
\hline 48 & barrel & tweezers & propeller & fountain \\
\hline 49 & funnel & bug & hoof & microscope \\
\hline 50 & stethoscope & banjo & igloo \\
\hline 51 & fingerprint & saxophone & tornado \\
\hline 52 & cap & squash & hourglass \\
\hline 53 & anvil & tape & tuning fork \\
\hline 54 & skateboard & towing & sangourine & sorting \\
\hline 55 & flocorder & te & \\
\hline
\end{tabular}




\section{Appendix E}

Department of Speech-Language Pathology

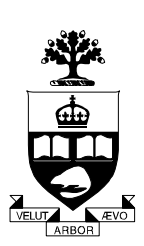

UNIVERSITY OF TORONTO FACULTY OF MEDICINE Rehabilitation Sciences Building 160-500 University Avenue Toronto, Ontario M5G 1 V7

Tel: (416) 978-2770

Fax: (416) 978-1596

E-mail: speech.path@utoronto.ca

\section{Information Letter and Informed Consent Form for Parents of Children in Pilot Study}

Title of Research Project: Vocabulary Development in Armenian Children Attending Bilingual Preschools

Investigator: Alice Hovsepian, $\mathrm{PhD}$ Student Department of Speech-Language Pathology, University of Toronto

The research project is designed as my $\mathrm{PhD}$ dissertation research under the supervision of $\mathrm{Dr}$. Carla J. Johnson.

\section{Dear Parent,}

This is a formal invitation to consider giving your consent for your child to participate in this study. Your child is qualified to take part in this study if he or she is 4- or 5-years old, and if at least one parent speaks Armenian with the child at home.

The decision to give your consent for your child to participate in this study is totally voluntary. You or your child can withdraw from the study at any time for any reason.

This study is part of the project. The purpose of this study is to develop a vocabulary test for preschool Armenian children. By developing this test we will be able to study the development of English and Armenian vocabulary in Armenian children. About eight children, four 4-year-old and four 5-year-old children will participate in this study. Half of these children will speak Western Armenian at home and half of them Eastern Armenian.

After I receive your consent, I will make arrangements with the principal to have a 1-hour session with your child at the Saturday school. After receiving your child's approval to participate, I will ask him/her to point to pictures that I name in Armenian or English and to name pictures in Armenian or English. This will take about 40 minutes. These pictures are all simple line-drawing pictures of different objects, animals, fruits or actions. These pictures will be presented to your child on a laptop computer. I will record your child's responses on the laptop. 
Participation in this study provides no direct benefits and presents no foreseeable risks. The only inconvenience that your child might face is feeling tired. If your child shows any sign of tiredness or simply mentions that he or she is tired, we will have a short break and continue with the task after the break, or at another time.

All information obtained from you and your child will be confidential. I will use a code for identifying each child in my database, and there will be no mention of names anywhere. The information I collect from your child by computer will be saved on the lab computer on a space protected by password. The information recorded on paper will be kept in a locked file in my supervisor's lab. No other person except me, my supervisor and possibly a research assistant will have access to this information. All of this information will be destroyed completely after five years following publication of the study. The paper data will be shredded and the computer data will be completely deleted.

The results obtained from all participants in this study will be reported as group findings in future publications with no mention of names or any other personal information. A summary of the results of the project will be prepared and sent to you.

If you have any further questions you can contact me, Alice Hovsepian, by phone at 416-9468638 or by e-mail at alice.hovsepian@utoronto.ca. For information regarding your rights as a participant you can contact the University of Toronto Ethics Review Office by phone at 416-9463273 or by e-mail at ethics.review@utoronto.ca.

If you are interested in permitting your child to participate in this study please provide your answers to the following questions:

Child's name: Gender: $\square$ Male $\square$ Female

Date of birth: Age: year /month/ day years; months

Which dialect of Armenian does your child speak? $\square$ Western Armenian $\square$ Eastern Armenian Do parents speak Armenian at home?

$\square$ Yes, at least one parent $\square$ No

If your child is either 4- or 5-years old, and if your answer to the last question is 'Yes', your child is qualified to take part in this study. Please print your name, provide your phone number, sign and date the last line of one of the two copies of this form. Then, put the signed form in the envelope provided with the form, seal the envelope, and give the envelope to your child to return it to the teacher who will pass it to me unopened. Please keep the second copy of the form for your own reference. 


\section{Investigator's Statement}

I have fully explained this study to the parent. I have answered all the questions that the parent asked.

Signature of the Investigator

Date:

\section{Parent's Consent}

I have read the information provided in this Informed Consent Form. All my questions have been answered satisfactorily. I agree to prticipate in this study and give my consent for my child's participation.

Name:

Your Phone Number:

Your Signature

Date: 


\section{Child Assent Script for Participants in Pilot Study}

The investigator presented the following script to the child participant in the language most comfortably used by the child at home. A positive answer to the first question was considered as an assent to participate in the tasks.

"We are going to look at some pictures. I will name some of the pictures and I will ask you to show them, or to say their names. I will record your voice when you name the pictures.

Do you want to look at the pictures? $\square$ Yes $\square$ No

Do you have any questions you would like to ask?" 


\section{Appendix F}

\section{Home Language Use Questionnaire}

In order to be able to better understand first and second language learning in children, we would like to obtain some information about your child's language use at home. Please complete the following questions concerning the child in this study.

Date:

Child's name: Gender: $\square$ Male $\square$ Female

Date of birth:

Age: year /month/ day Age on entering preschool:

years; months years; months

School name: Preschool group: $\square$ Junior Kindergarten $\square$ Senior Kindergarten

Armenian teacher: English teacher:

Which preschool program(s) has the child attended during the pervious years?

$\square$ Nursery $\square$ Junior Kindergarten $\square$ Senior Kindergarten $\square$ None

Your relationship to the child: $\square$ Mother $\square$ Father $\square$ Other:

Home postal code: Home phone number:

Father's age: Father's total years of education: Father's total years in Canada:

Father's first language: $\square$ Eastern Armenian $\square$ Western Armenian $\square$ English $\square$ Other:

What language(s) does the father use with the child? __ $\%$ Armenian __ $\%$ English __ \%Other Mother's age: ___ Mother's total years of education: Mother's total years in Canada:

Mother's first language: $\square$ Eastern Armenian $\square$ Western Armenian $\square$ English $\square$ Other:

What language(s) does the mother use with the child? _ $\%$ Armenian __ English _ \%Other What language(s) does the child use in each of the following settings?

1. With his/her father

2. With his/her mother

3. With his/her siblings

4. With his/her grandparents

5. With his/her playmates

6. When watching TV/Video
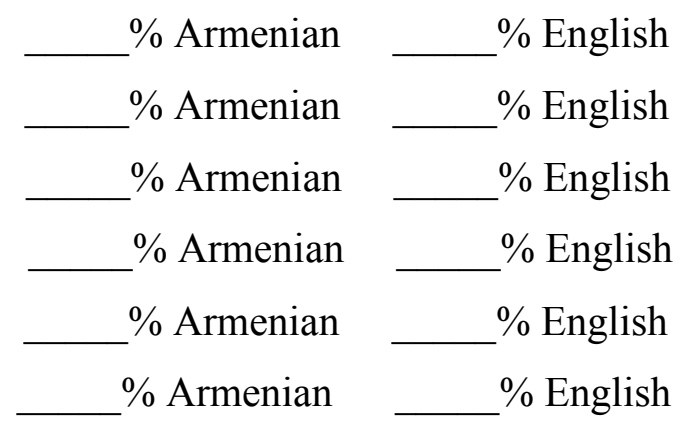
$\%$ Other $\%$ Other $\%$ Other $\%$ Other $\%$ Other $\%$ Other

7. In general, what language(s) does the child use most of the time at home? $\%$ Armenian $\%$ English $\%$ Other

8. At what age did the child start learning Armenian?

9. At what age did your child start learning English? 
10. In general, in which language do you think your child communicates better?

$\square$ Western Armenian $\quad \square$ Eastern Armenian $\quad \square$ English $\square$ Other:

11. In our family it is important that our child speak Armenian proficiently.

$\square$ Strongly agree $\quad \square$ Agree $\quad \square$ Disagree $\quad \square$ Strongly disagree

12. In our family it is important that our child speak English proficiently.

$\square$ Strongly agree $\quad \square$ Agree $\quad \square$ Disagree $\quad \square$ Strongly disagree

13. In our family it is important that our child have good reading and writing skills in Armenian.

$\square$ Strongly agree $\quad \square$ Agree $\square$ Disagree $\square$ Strongly disagree

14. In our family it is important that our child have good reading and writing skills in English.

$\square$ Strongly agree $\quad \square$ Agree $\quad \square$ Disagree $\quad \square$ Strongly disagree

15. In our family it is important that our child consider him/herself as an Armenian.

$\square$ Strongly agree $\quad \square$ Agree $\square$ Disagree $\square$ Strongly disagree

16. In our family it is important that our child consider him/herself as a Canadian.

$\square$ Strongly agree $\quad \square$ Agree $\square$ Disagree $\quad \square$ Strongly disagree

17. What family activities help your child to develop an Armenian identity?

18. What family activities help your child to develop a Canadian identity?

19. What were your reasons for sending your child to Armenian school?

20. At what age did your child say his/her first words?

21. At what age did your child combine words to make two- or three-word sentences?

22. Do others easily understand your child's speech? $\square$ Yes $\square$ No

23. Do you have any concerns about your child's verbal communication skills? $\square$ Yes $\square$ No If your answer is 'Yes', please explain.

Thank you for completing this questionnaire. Your cooperation is greatly appreciated. We look forward to sharing the findings of this study with you. 


\section{Appendix G}

Department of Speech-Language Pathology

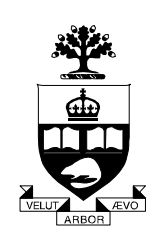

UNIVERSITY OF TORONTO

FACULTY OF MEDICINE

Rehabilitation Sciences Building

160-500 University Avenue

Toronto, Ontario M5G 1 V7

Tel: (416) 978-2770

Fax: (416) 978-1596

E-mail: speech.path@utoronto.ca

\section{Information Letter and Informed Consent Form for Parents of Children in Study 2 Time 1}

Title of Research Project: Vocabulary Development in Armenian Children Attending Bilingual Preschools

Investigator: Alice Hovsepian, PhD Student

Department of Speech-Language Pathology, University of Toronto

This study is designed as my $\mathrm{PhD}$ dissertation research under the supervision of Dr. Carla J. Johnson.

\section{Dear Parent,}

This is a formal invitation to consider giving your consent for you and your child to participate in this study. You and your child are qualified to take part in this study if your child is 4- or 5-years old, and if at least one parent speaks Armenian with the child at home.

Participation in this study is voluntary. You or your child can withdraw from the study at any time during the study for any reason.

The purpose of this study is to see how Armenian children learn word meanings in Armenian and English during the years that they attend kindergarten. In addition, I want to find out what factors are related to word learning in children. Overall, about 20 Junior Kindergarten and 20 Senior Kindergarten children who attend the Armenian day schools in Toronto and their parents will participate in this study. To participate, at least one parent must speak Armenian at home with the child.

If you agree to participate in this study, please fill out the questionnaire you have received with this letter. In this questionnaire you will be asked questions about your child's age, gender, the preschool program that he or she is attending, your language and education, and the language that the child uses with different people and during different activities at home. This questionnaire will take about 15 minutes to complete. 
Your child will take part in two test sessions. The first session will be done in October, and the second in May. At both times your child will be tested on two separate days within a week after receiving his/her approval to participate. On the first day, I will give a vocabulary test. During this test I will ask your child to point to pictures that I name in Armenian and to name pictures in Armenian. On this day a memory test will also be given. During this test your child will be asked to listen to some taped words one at a time and to repeat them. On the second day, your child will be asked to point to pictures that I name in English and to name pictures in English. On this day a test of nonverbal reasoning will also be given. During this test your child will be asked to put some picture cards into their slots in a frame. Each test day will take $45-55$ minutes. At the second time of testing in May only the vocabulary tasks will be given. On the first day the Armenian and on the second day the English vocabulary tasks will be given. Each test day will take about 30 minutes. During these test sessions I will show the pictures on a laptop computer and I will record your child's voice while naming the pictures or repeating the words.

Participation in this study provides no direct benefits and presents no foreseeable risks. The only inconvenience that your child might face is feeling tired. If your child shows any sign of tiredness or simply mentions that he or she is tired, we will have a short break and continue with the task after the break, or on the following day.

All information obtained from you and your child will be confidential. I will use a code for identifying each child and parent in my database, and there will be no mention of names anywhere. The information I collect from your child by computer will be saved on the lab computer on a space protected by password. The information recorded on paper will be kept in a locked file in my supervisor's lab. No other person except me, my supervisor and possibly a research assistant will have access to this information. All of this information will be destroyed completely after five years following publication of the study. The paper data will be shredded and the computer data will be completely deleted.

The results obtained from all participants in this study will be reported as group findings in future publications with no mention of names or any other personal information. A summary of the results of the project will be prepared and sent to you.

If you have any further questions you can contact me, Alice Hovsepian, by phone at 416-9468638 or by e-mail at alice.hovsepian@utoronto.ca. For information regarding your rights as a participant you can contact the University of Toronto Ethics Review Office by phone at 416-9463273 or by e-mail at ethics.review@utoronto.ca.

This letter is a formal request seeking your consent for filling out the questionnaire and for letting your child participate in the study. If you are interested in participating in this study please provide your answer to the following question:

Do parents speak Armenian at home?

$\square$ Yes, at least one parent $\quad \square$ No

If your answer to the above question is 'Yes', you and your child are qualified to participate in this study. Please print your name, provide your phone number, sign and date the last line of one of the two copies of this form. Then, put the signed form in the envelope provided with the form, 
seal the envelope, and give the envelope to your child to return it to the teacher who will pass it to me unopened. Please keep the second copy of the form for your own reference.

\section{Investigator's Statement}

I have fully explained this study to the parent. I have answered all the questions that the parent asked.

Signature of the Investigator Date:

\section{Parent's Consent}

I have read the information provided in this Informed Consent Form. All my questions have been answered satisfactorily. I agree to prticipate in this study and also give my consent for my child's participation.

Name:

Your Phone Number:

Your Signature Date: 
Department of Speech-Language Pathology

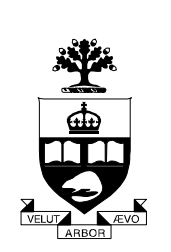

UNIVERSITY OF TORONTO

FACULTY OF MEDICINE

Rehabilitation Sciences Building

160-500 University Avenue

Toronto, Ontario M5G 1V7

Tel: (416) 978-2770

Fax: (416) 978-1596

E-mail: speech.path@utoronto.ca

\section{Information Letter and Informed Consent Form for Parents of Children in Study 2 Time 2}

Title of Research Project: Vocabulary Development in Armenian Children Attending Bilingual Preschools

Investigator: Alice Hovsepian, PhD Student

Department of Speech-Language Pathology, University of Toronto

This study is designed as my $\mathrm{PhD}$ dissertation research under the supervision of Dr. Carla J. Johnson.

\section{Dear Parent,}

This is a reminder that the second time of testing will start in April. As already mentioned in the initial Information Letter and Consent form, your child will be tested for the second and final time. During this test I will ask your child to point to pictures that I name in Armenian and English and also to name pictures in Armenian and English. In addition, a hearing test will be given. During this test your child's hearing will be screened. To do this test your child will be asked to wear small headphones, and the response of the ears to sound will be automatically recorded. This test will take about 5 minutes. The results of this test will help us identify children who might have hearing problems. If so, the parents will be informed of the test results and referral to an ear specialist will be suggested.

In addition, to obtain a better understanding of your child's hearing we would appreciate your answer to the following question:

Did your child have any ear problems (such as ear infection or fluid emissions) in the last 8 months? $\square$ Yes $\square$ No

If the answer is 'Yes' please explain:

When did your child have the problem?

What was the problem?

If you have any further questions you can contact me, Alice Hovsepian, by phone at 416-9468638 or by e-mail at alice.hovsepian@utoronto.ca. For information regarding your rights as a participant you can contact the University of Toronto Ethics Review Office by phone at 416-9463273 or by e-mail at ethics.review@utoronto.ca. 
Please print your name, provide your phone number, sign and date the last line of one of the two copies of this form. Then, put the signed form in the envelope provided with the form, seal the envelope, and give the envelope to your child to return it to the teacher who will pass it to me unopened. Please keep the second copy of the form for your own reference.

\section{Investigator's Statement}

I have fully explained this study to the parent. I have answered all the questions that the parent asked.

Signature of the Investigator

Date:

\section{Parent's Consent}

I have read the information provided in this Information letter. All my questions have been answered satisfactorily. I agree to participate in this study and also give my consent for my child's participation.

Name:

Your Phone Number:

Your Signature Date: 


\section{Child Assent Script \\ for Participants in Study 2 \\ Time 1 - Day 1}

The investigator presented the following script to the participant in the language most comfortably used by the child at home. A positive answer to the first question was considered as an assent to participate in the tasks.

"We are going to do three things today. First we will look at some pictures. I will name a picture and will ask you to point to it. Then, I will show you a picture and will ask you to say its name. I will record your voice when you name the pictures. At the end, we will listen to some new words on my computer. When you hear a new word, you should repeat that word. I will record your voice when you say the new words.

Do you want to do these things together? $\square$ Yes $\square$ No

Do you have any questions you would like to ask?" 


\section{Child Assent Script \\ for Participants in Study 2 \\ Time 1 - Day 2}

The investigator presented the following script to the participant in the language most comfortably used by the child at home. A positive answer to the first question was considered as an assent to participate in the tasks.

"Today we are also going to do three things. First we will look at some pictures. I will name a picture and will ask you to point to it. Then, I will show you a picture and will ask you to say its name. I will record your voice when you name the pictures. At the end, we will play a game with picture cards and rubber shapes. I will give you the cards or shapes and you will put them in their places.

Do you want to do these things together? $\square$ Yes $\quad \square$ No

Do you have any questions you would like to ask?" 


\section{Child Assent Script \\ for Participants in Study 2 \\ Time 2 - Day 1}

The investigator presented the following script to the participant in the language most comfortably used by the child at home. A positive answer to the first question was considered as an assent to participate in the tasks.

"We are going to do two things today. First we will look at some pictures. I will name a picture and will ask you to point to it. Then, I will show you a picture and will ask you to say its name. I will record your voice when you name the pictures.

Do you want to do these things together? $\square$ Yes $\quad \square$ No

Do you have any questions you would like to ask?" 


\section{Child Assent Script \\ for Participants in Study 2 \\ Time 2 - Day 2}

The investigator presented the following script to the participant in the language most comfortably used by the child at home. A positive answer to the first question was considered as an assent to participate in the tasks.

"Today we are going to do three things. First we will look at some pictures. I will name a picture and will ask you to point to it. Then, I will show you a picture and will ask you to say its name. I will record your voice when you name the pictures. At the end, we will test your ears and find out how well you hear different sounds. You will sit on the chair and I will place these (while showing the probes) two tiny headphones in your ears. Then you will hear some noises in your ears and we will see on this screen (while showing the screen which monitors the results) how your ears hear.

Do you want to do these things together? $\square$ Yes $\quad \square$ No

Do you have any questions you would like to ask?" 


\section{Appendix $\mathrm{H}$}

\section{Hebrew Pseudo-word Phonological Short-Term Memory Task}

(Phonetic Transcription) $^{11}$

\begin{tabular}{|c|c|c|c|}
\hline 2-syllable & & 3-syllable & \\
\hline eckla & $\backslash \partial \mathrm{k}_{\mid} \mid a ̈ h \backslash$ & kamasher & 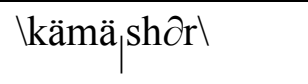 \\
\hline doogut & $\backslash d \ddot{u}$ gät $\backslash$ & maldera & $\backslash$ mäld $\partial$ räh $\backslash$ \\
\hline palake & $|p a ̈| l \mid k \backslash$ & pashmaloot & |päshmä|lüt\} $\\
{\hline \text { lived }} &{\backslash \operatorname{li}_{\mid} \mathrm{v} \partial \mathrm{d} \backslash} &{\text { hadgasa }} &{\text { \hädgä } \text { säh\ }} \\
{\hline \text { boshen }} &{\backslash \text { bosh } \partial \mathrm{n}} &{\text { nitbaseem }} &{||_{\text {nitba }} \operatorname{sim}} \\
{\hline \text { kamuff }} &{\backslash_{\mathrm{kä}} \mathrm{mäf}} &{\text { tolabeem }} &{\text { 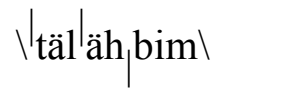 }} \\
{\hline \text { kisroo }} &{\backslash \text { kis } r u ̈ \} &{\text { kiloton }} &{\backslash \text { kilä,ton } \backslash} \\
{\hline \text { pofek }} &{\backslash p \operatorname{pof} \partial \mathrm{k} \backslash} &{\text { Mudbeitok }} &{\backslash \text { mädb } \partial_{\mid} \text {tok } \backslash} \\
{\hline \text { 4-syllable }} &{ } &{\text { 5-syllable }} &{ } \\
{\hline \text { batahpoofeem }} &{\text { 〈bätäpü,fim \} } &{\text { efroloteihem }} &{\backslash \partial \text { frolot } \partial \mathrm{i} \mid \mathrm{h} \partial \mathrm{m} \backslash} \\
{\hline \text { nitparsegeem }} &{\lfloor\text { nit } \mid \text { pärs } \partial \text { gim } \backslash} &{\text { meihatavikot }} &{\text { 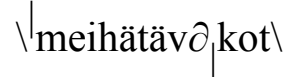 }} \\
{\hline \text { hitnavaloot }} &{\backslash \text { hit } \mid \text { nävo,lüt } \backslash} &{\text { makataforum }} &{\backslash \text { mäkä|täfei räm\ }} \\
{\hline \text { mitargenill }} &{\backslash \mathrm{m} \partial \mid \text { tärg} \partial_{\mid} \text {nol } \backslash} &{\text { beitahasoofum }} &{\backslash \text { beithä|äls } \partial_{\mid} \text {fäm } \backslash} \\
{\hline \text { betiplooshug }} &{|\mathrm{b} \partial t i p l u ̈| s h a ̈ g \backslash} &{\text { lesharneigoleem }} &{\text { 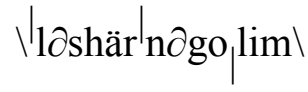 }} \\
{\hline \text { histargebut }} &{\backslash \text { his } \mid \text { tärg } \partial \text { büt } \backslash} &{ } &{ } \\
\hline\end{array}$
\end{tabular}

11 Key: $\quad \ddot{a}$ as in hard; $\ddot{u}$ as in loot; $\partial$ schwa as in echo;

$\mathrm{I}^{\mathrm{X}}$ indicates primary stress; $\left.\right|_{\mathrm{X}}$ indicates secondary stress 


\section{Task Administration Instructions}

This task is administered individually. During the task, the participant listens to the tape-

recorded "pseudo-words" presented one at a time and is instructed to repeat each pseudo-word immediately and completely. There is no stopping rule for this task. 


\section{Appendix I}

Department of Speech-Language Pathology

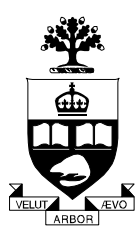

UNIVERSITY OF TORONTO

FACULTY OF MEDICINE

Rehabilitation Sciences Building

160-500 University Avenue

Toronto, Ontario M5G 1V7

Tel: (416) 978-2770

Fax: (416) 978-1596

E-mail: speech.path@utoronto.ca

\section{Informed Consent Form for Teachers in Classroom Observation}

Title of Research Project: Vocabulary Development in Armenian Children Attending Bilingual Preschools

Investigator: Alice Hovsepian, PhD Student

Department of Speech-Language Pathology, University of Toronto

The research project is designed as my PhD dissertation research under the supervision of Dr. Carla J. Johnson.

\section{Dear Teacher,}

This is a formal invitation to consider giving your consent for you and your class to participate in this study. The decision to take part in this study is totally voluntary. You can withdraw from the study at any time for any reason.

This study is part of my Ph.D. project on vocabulary learning. The purpose of this part of the study is to observe the Junior and Senior Kindergarten classrooms in Armenian day schools in Toronto. The focus of these observations is on the languages used in the classroom by the teacher and/or by students. Overall, four classrooms will be observed. Each classroom will be observed at four points in time. The first observation will be in September. Following that, the next three observations will be done every other month. Each observation session will take one hour.

Upon giving your consent to participate in this study, we will arrange a 1-hour observation session at your convenience. On our first meeting before starting with the observation I will ask you few questions. These questions will be your name, the language that you teach, and the languages that you speak. During the observation I will sit at the rear of the classroom in a place that will cause the least distraction for the class. I will use a checklist to record the observations. The observations will be on the frequency of the use of Armenian and/or English by you and/or your students.

Participation in this study provides no direct benefits and presents no foreseeable risks. The only inconvenience that you might face is the distraction that my presence might bring to the class. To minimize this distraction I will sit at the rear of the class during the whole observation session and I will not talk to you or your students during this period. 
All information obtained from you and your classroom will be confidential. I will use a code for identifying you and your classroom in my database, and there will be no mention of names anywhere. The information will be saved on the lab computer on a space protected by password. The information recorded on paper will be kept in a locked file in my supervisor's lab. No other person except me, my supervisor and possibly a research assistant will have access to this information. All of this information will be destroyed completely after five years following publication of the study. The paper data will be shredded and the computer data will be completely deleted.

The results obtained from all classrooms in this study will be reported as group findings in future publications with no mention of names or any other personal information. A summary of the results of the project will be prepared and sent to you.

If you have any further questions you can contact me, Alice Hovsepian, by phone at 416-9468638 or by e-mail at alice.hovsepian@utoronto.ca. For information regarding your rights as a participant you can contact the University of Toronto Ethics Review Office by phone at 416-9463273 or by e-mail at ethics.review@utoronto.ca.

If you decide to participate, please print your name, sign and date the last line of one of the two copies of this form, and return it to me in the envelope provided with the form. Please keep the second copy of the form for your own reference.

\section{Investigator's Statement}

I have fully explained this study to the teacher. I have discussed the activities and have answered all the questions that the teacher asked.

Signature of the Investigator Date:

\section{Participant's Consent}

I have read the information provided in this Informed Consent Form. All my questions have been answered satisfactorily. I agree to prticipate in this study.

Name:

Your Signature Date: 

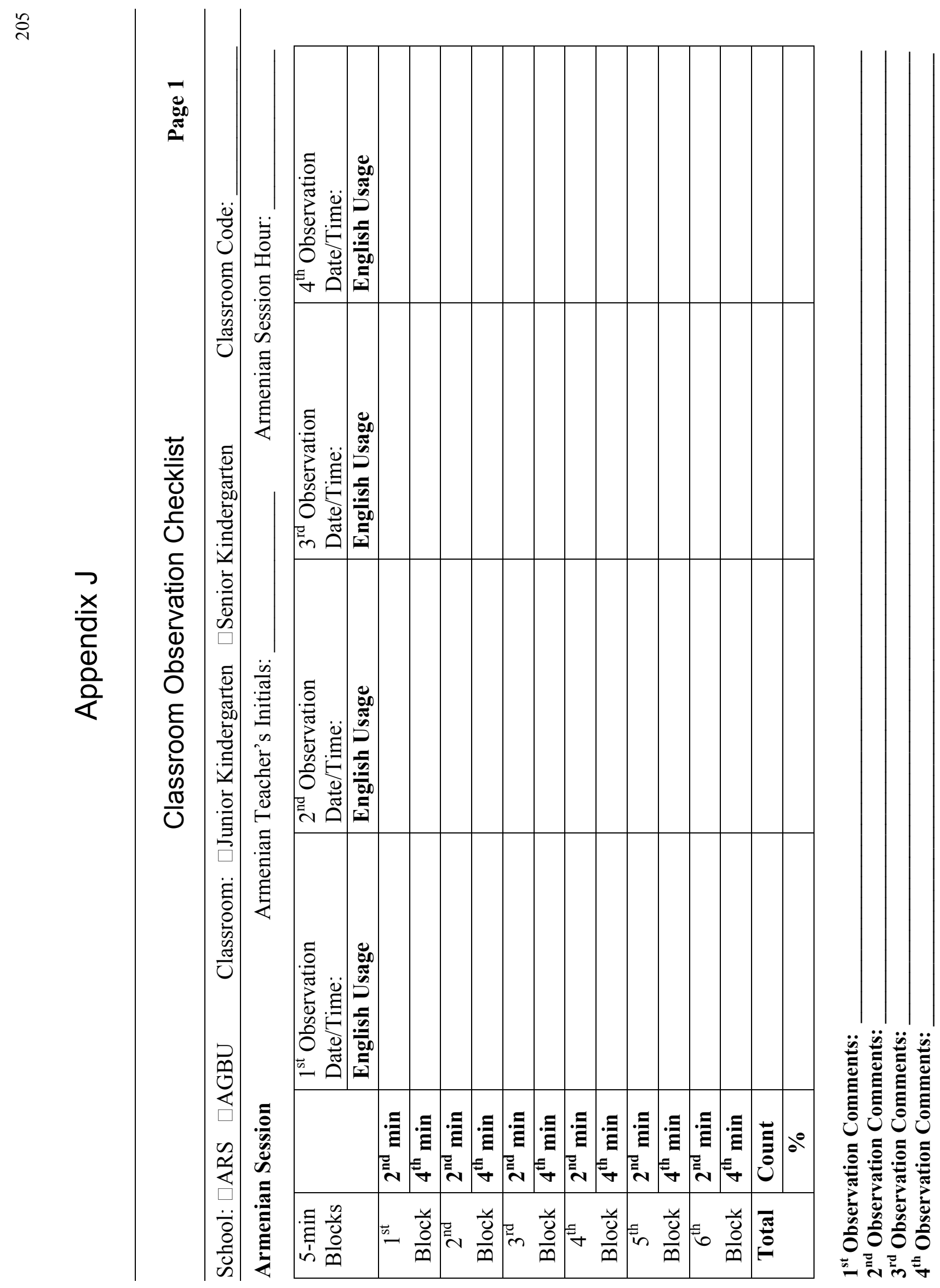
ปั
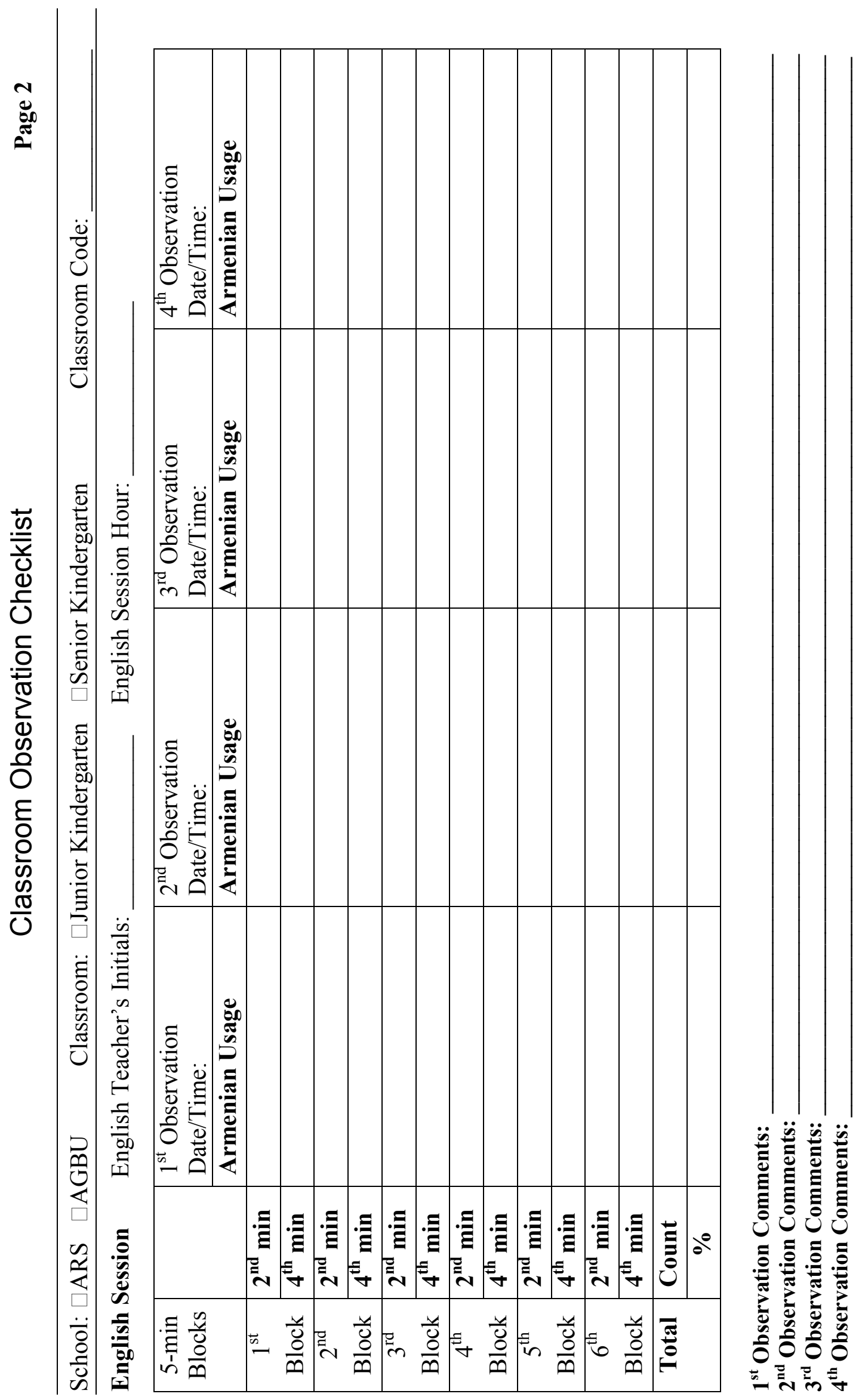


\section{Instructions}

The columns labeled $1^{\text {st }}$ Observation, $2^{\text {nd }}$ Observation, $3^{\text {rd }}$ Observation, and $4^{\text {th }}$ Observation are used for recording four times of observations, two months apart from each other. At each observation time each classroom is observed twice within a day, one for Armenian session and the other for English session. Page 1 is used for recording the Armenian session observations and Page 2 is used for recording the English session observations. Each observation session lasts half-an-hour. Each half-an-hour observation session is divided into six 5-minute observation blocks shown in the first column. During the second and fourth 1-minute of each block, the instances of use of English during Armenian session or use of Armenian during English session are tallied in the spaces provided. These instances include but are not restricted to use of single words, phrases or sentences. A stop-watch is used to keep track of the timing blocks. At the end, the total number and percentage of instances of use of the language other than the language of instruction is calculated. 